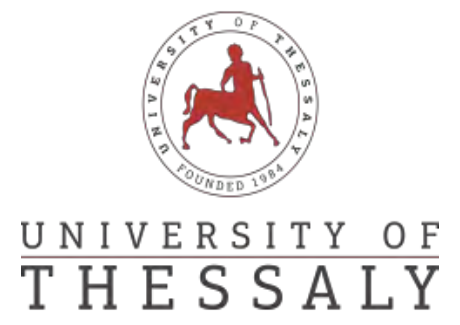

University of Thessaly

Department of Early Childhood Education

\title{
The Relations between Parental Support of Learning, Parental Attributes and Development of Self-Regulated Learning Skills in Young Children
}

\author{
Submitted by Eleni Kallia as a thesis for the degree of Doctor of \\ Philosophy in the Department of Early Childhood Education, University of \\ Thessaly
}

Volos, 2018

The dissertation was partially supported by a scholarship from the State Scholarships Foundation (IKY) funded by the Action "Program for Granting Post Graduate Studies of Second Cycle" from resources of the Operational Program "Human Resources Development, Education and Lifelong Learning", 2014-2020 co-funded by the European Social Fund and the Greek Public State. 


\section{The Relations between Parental Support of Learning, Parental Attributes and Development of Self-Regulated Learning Skills in Young Children}

Submitted by Eleni Kallia as a thesis for the degree of Doctor of Philosophy in the Department of Early Childhood Education, University of Thessaly

\section{Supervising Committee (three-member)}

Dermitzaki Irini, Department of Early Childhood Education, University of Thessaly (Supervisor)

Bonoti Fotini, Department of Early Childhood Education, University of Thessaly

Nisiotou Ioulia, Department of Special Education, University of Thessaly

\section{Examination Committee (seven-member)}

Dermitzaki Irini, Department of Early Childhood Education, University of Thessaly (Supervisor)

Bonoti Fotini, Department of Early Childhood Education, University of Thessaly

Nisiotou Ioulia, Department of Special Education, University of Thessaly

Gonida Eleftheria, Department of Psychology, Aristotle University of Thessaloniki

Kafetsios Konstantinos, Department of Psychology, University of Crete

Misailidi Plousia, Department of Primary Education, University of Ioannina

Botsoglou Kafenia, Department of Special Education, University of Thessaly

Volos, 2018 


\begin{abstract}
This study investigated the relations between maternal supportive and contingent behaviours during mother-child problem-solving and children's self-regulation of learning (SRL). The purpose of this study was to explore multiple dimensions of maternal support and their relations with specific SRL skills in young children both concurrently and longitudinally. Children's and mothers' demographic and general characteristics were also taken into account (children's age and gender, maternal education, maternal self-efficacy and parenting style and children's temperament).

Two pilot studies were conducted to test the appropriateness of the measures. The main study was conducted two times with a six-month interval between the two phases. The participants were thirty-five preschool children with their mothers. The children's age ranged from 54 to 72 months old. Children were first asked to solve Visual-spatial and Language tasks independently and then with their mothers. The structured observation method was used to record children's SRL skills, performance and maternal supportive behaviours during problem-solving, through a micro-analytic approach. Children's Cognitive-Metacognitive skills, Emotional-Motivational skills and Autonomy skills were coded during the child-alone problem-solving. A structured observation form was employed to record different maternal behaviours that support the child during joint problem-solving, including aspects of Cognitive-Metacognitive support, Emotional-Motivational support and Autonomy support. Maternal Contingency was also taken into account to assess the extent to which the mother provided support to the child according to the level of his/her understanding of the child's need. The relations between the maternal supportive behaviours, children's SRL skills and cognitive performance were tested both cross-sectionally and longitudinally, from Time 1 to Time 2, to explore whether early maternal supportive behaviours contribute to children's later SRL skills and cognitive performance.

The results highlighted two aspects of maternal support that significantly predicted children's SRL skills and cognitive performance. At Time 1, maternal Autonomy support and Contingency were the most important maternal dimensions that predicted different aspects of children's SRL skills. At Time 2 maternal Autonomy support had a more prominent role in predicting children's Cognitive-Metacognitive, Emotional-Motivational and Autonomy skills. The same maternal practices emanated in the short-term longitudinal analysis but only for the Visual-spatial domain. Children's age was in some cases an important predictor of children's
\end{abstract}


SRL skills. Mediation analyses pointed out that children's Cognitive and Metacognitive skills mediated the effect of these two maternal dimensions on children's cognitive performance.

The present study contributes to methodological, theoretical, and practical issues. Few studies have investigated the relations in question both longitudinally and at a micro-level. This study highlights the importance of adopting a multidimensional approach when examining the role of maternal behaviours in children's learning also considering other dimensions, such as the nature of the task at hand. The study extends our understanding of the relationships between children's SRL skills and parenting behaviours that are activated early in children's learning career. Such findings could be taken into account by interventions that focus on fostering children's learning skills and promoting positive parent-child interactions and effective teaching methods both for parents and teachers. 


\section{Abstract (Greek Version)}

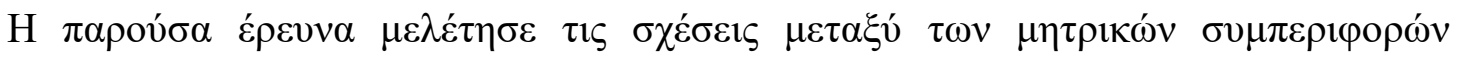

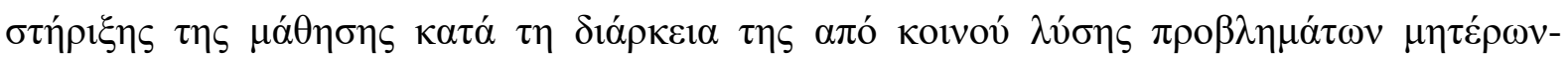

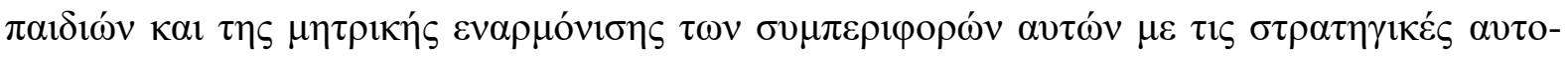

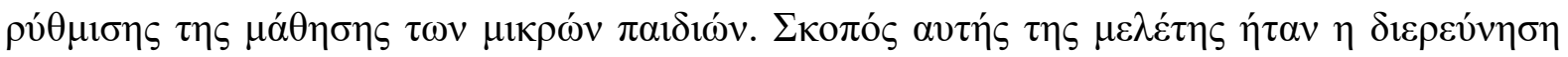

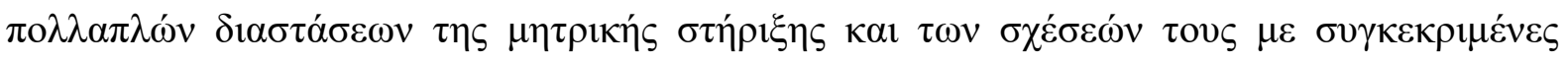

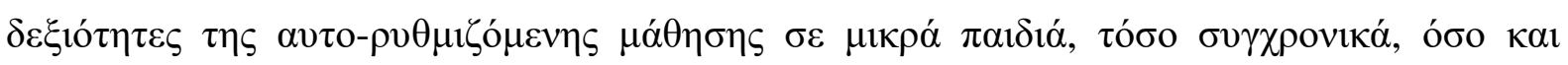

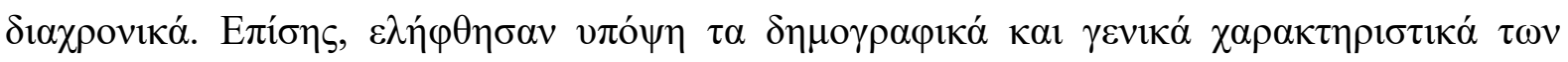

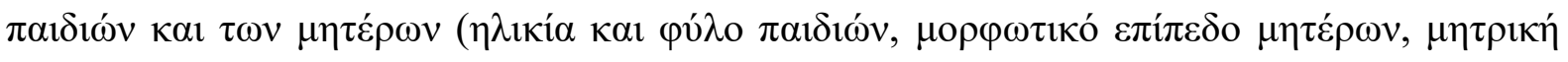

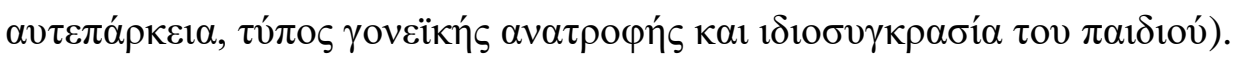

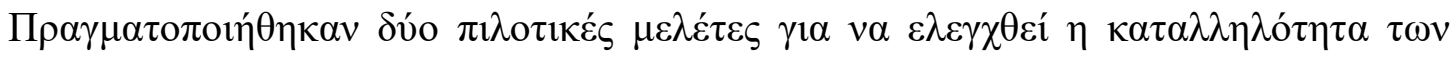

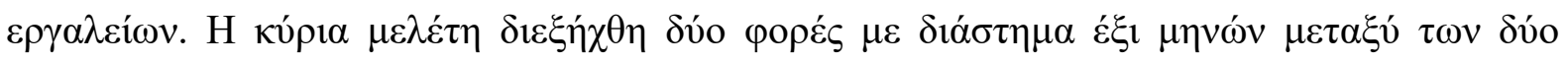

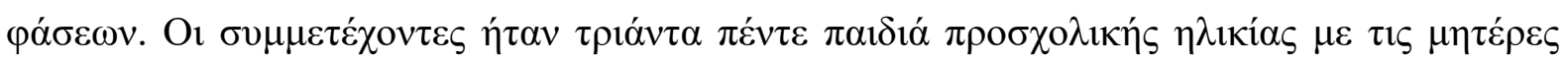

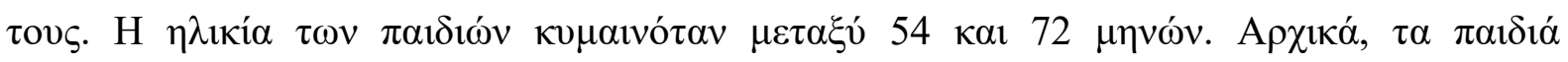

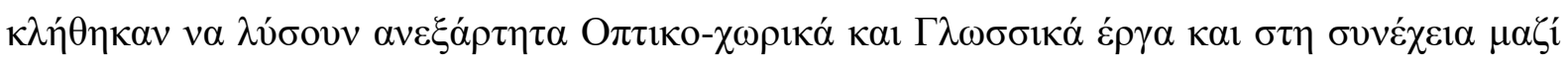

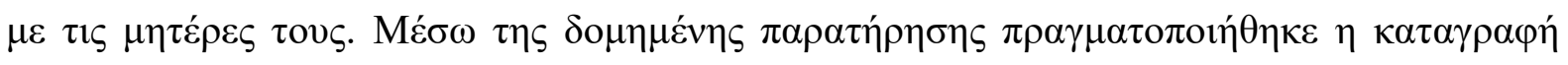

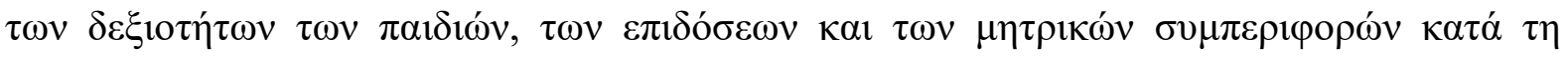

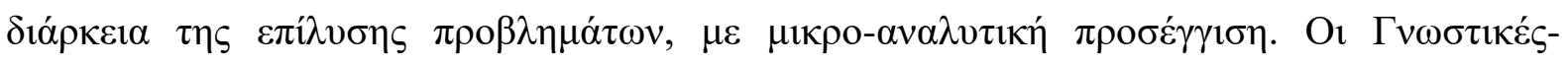

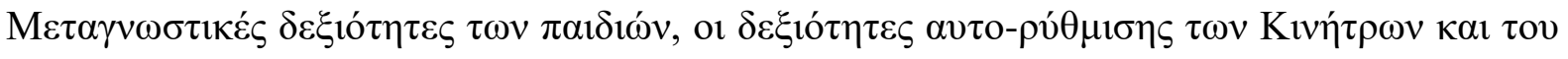

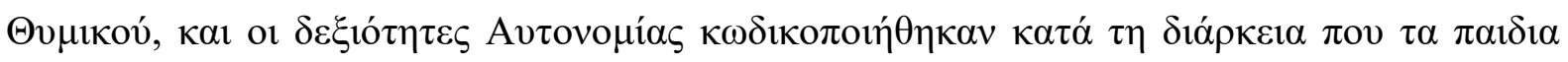

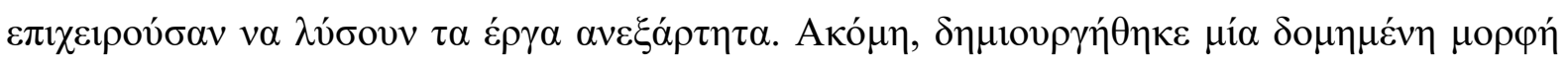

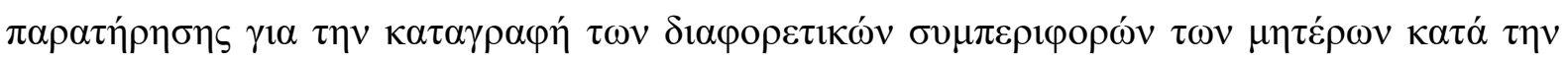

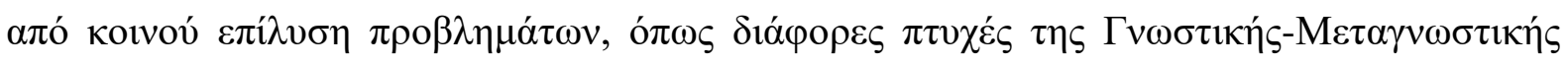

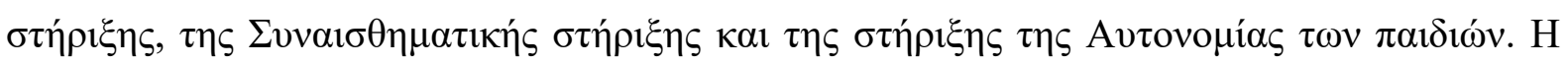

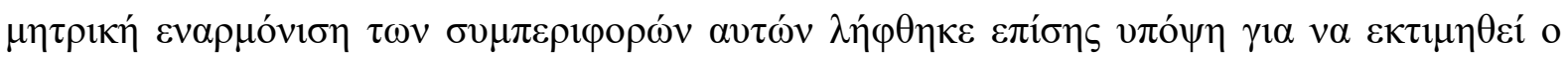

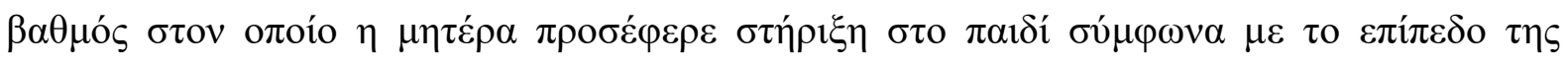

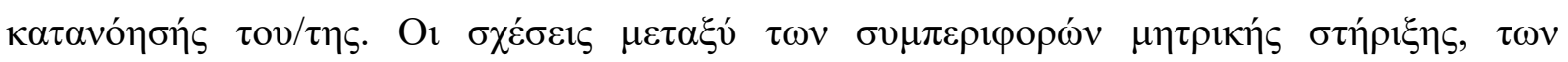

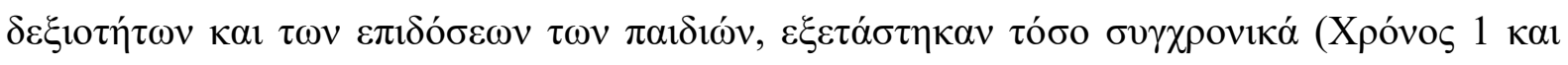

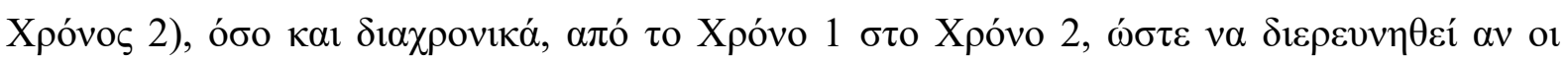

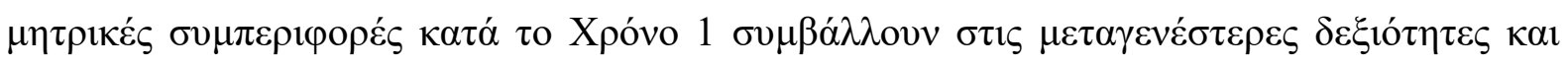

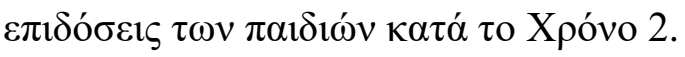

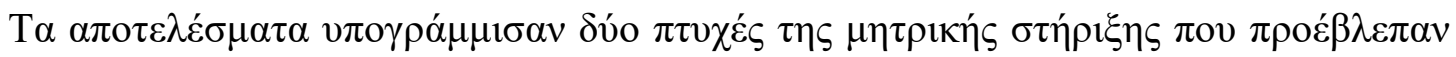

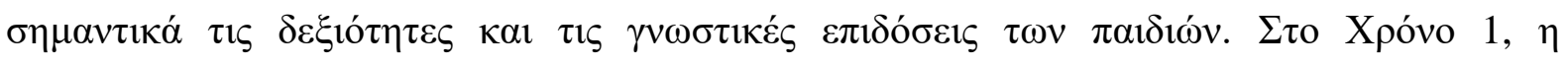




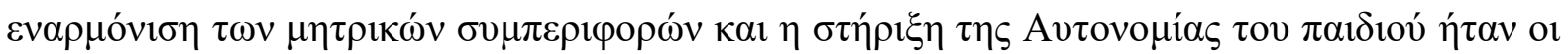

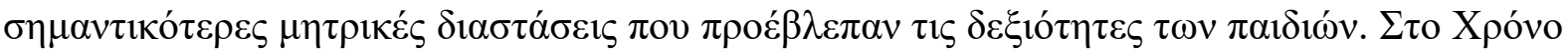

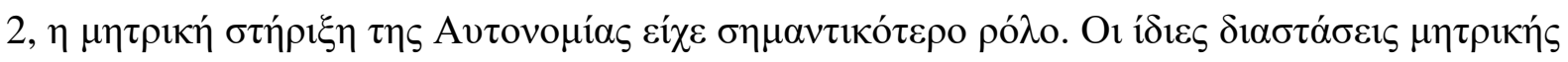

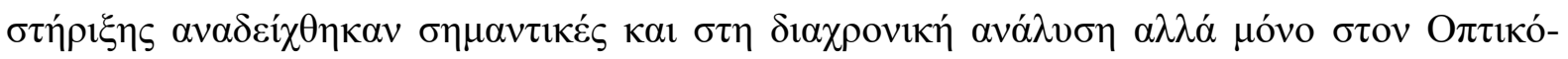

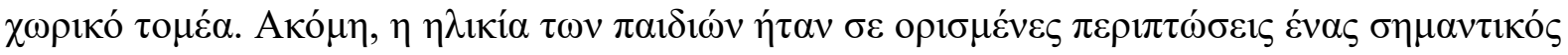

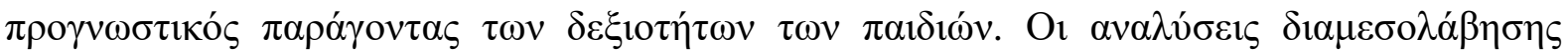

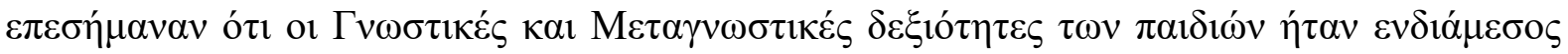

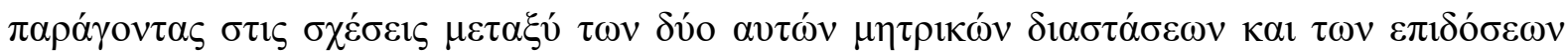
$\tau \omega v \pi \alpha \iota \delta ı \omega ́ v$.

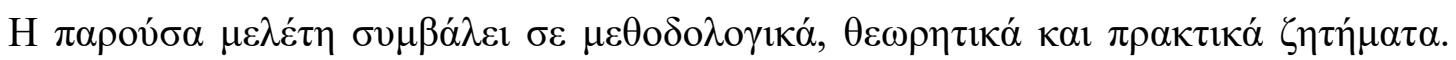

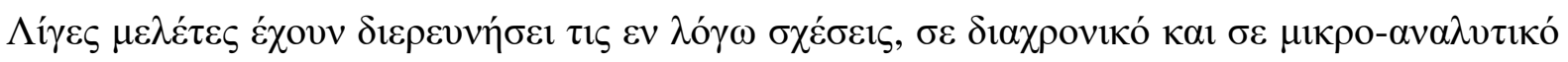

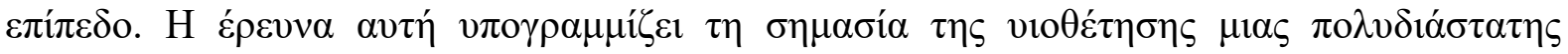

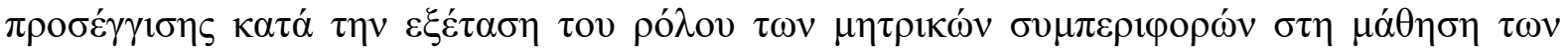

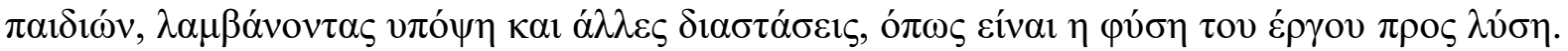

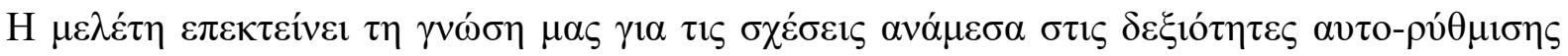

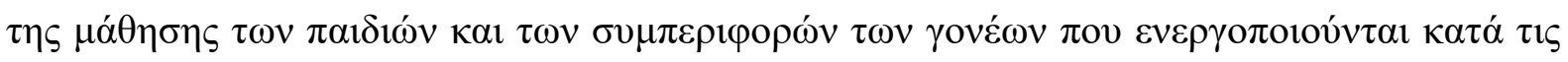

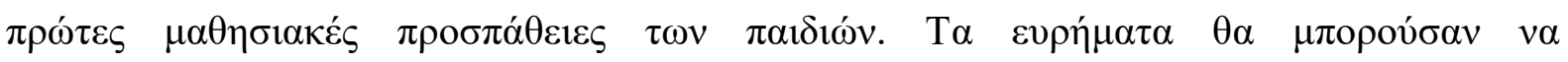

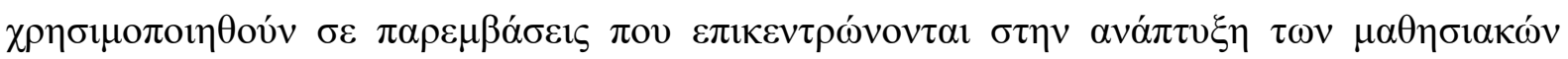

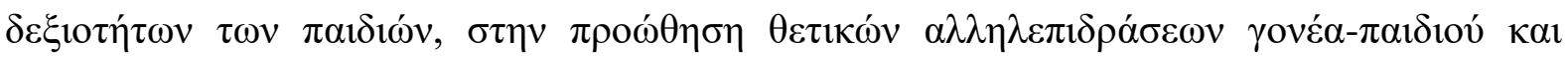

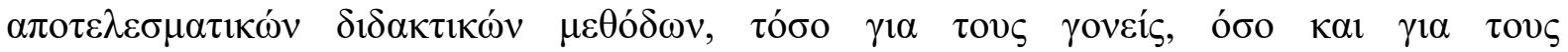

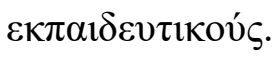


To my niece, Maria, who was patiently waiting for me, while I was working on my thesis, in order to spend some time together playing and dancing. 


\section{Acknowledgements}

A $\mathrm{PhD}$ is a long journey of feelings and thoughts. Feelings of frustration, disappointment and anxiety are usually followed by feelings of excitement, satisfaction and pride. Fortunately, during this journey, I have been accompanied by people who supported me and believed in me. Therefore, expressing my gratitude is the least I can do for them.

I am afraid that whatever I say about my supervisor, Professor Irini Dermitzaki could not portray what I really feel and think about her supportive, leading, and encouraging stance during all these years. I deeply move emotionally when I reflect on her tireless advice, consultations and assistance. Her academic knowledge and orientation, her valuable experience, strategies and approaches to various challenges have been a true inspiration for me, and I am confident that parts of them have been imprinted in the way I currently think and work. I feel grateful for all the time she dedicated to me, for her transmission of scientific knowledge, for her supportive behaviour, for believing in me. Without her assistance and guidance, this journey could not be completed. I hope her mentorship to continue in the years to come.

I would also like to express my appreciation to the Professor Fotini Bonoti, for all the advice on my thesis, her time, her interest and supportive role. Her suggestions were a significant part for the completion of this dissertation. I also express my deepest thanks to the Assistant Professor Ioulia Nisiotou, for without her help I would not be in this position today. Her advice many years ago guided me to stand at this specific point in my academic life. I appreciate her willingness to help me and support me in every stage of this research.

I feel the need to express my appreciation to the Associate Professor Eleftheria Gonida, for her interest and valuable advice in my work all these years and to Professor Konstantinos Kafetsios for his comments and suggestions and the time he devoted to my thesis. I am also thankful to the Associate Professor Plousia Misailidi and to Professor Kafenia Botsoglou for accepting to participate as members of the examination committee and for the time they allocated to my thesis.

I own many thanks to the mothers, children and teachers of the kindergartens, who participated in the study. Without their cooperation and their willingness to contribute, this thesis could not be implemented in the first place.

In the last part, I want to express my gratitude to the most important people in my life. First, to my husband Charis Konstas who had patiently listened and answered to my eternal concerns and for his unique reassuring way to approach the situations realistically. To my 
parents, Pantelis and Maria Kallia, for their confidence in me, their encouragement and support and their constant motivation to move forward. My sister Marietta and her husband Marinos Kintis for their valuable, lengthy academic discussions, for the sharing of knowledge, for their advice. To my brothers and their wives, Lefteris and Martha, Jiannis and Alexandra, for taking the time to listen to my worries and for taking care to provide me with some relaxing moments. Finally, to my close friends, Valia Siskopoulou, Christina Karavide, Georgios Kalamogiorgos, and Christina Karela for their high levels of understanding, who, despite my limited availability, they were always there for me, making this journey easier. 


\section{Table of Contents}

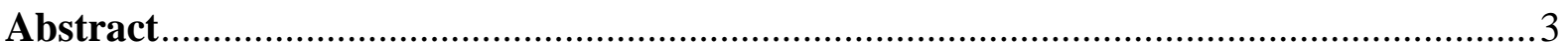

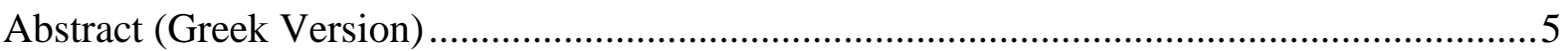

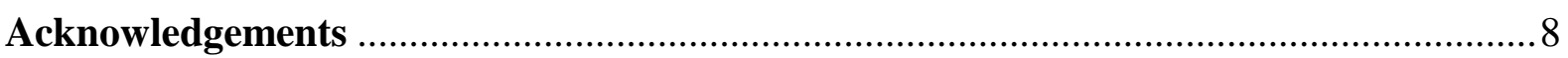

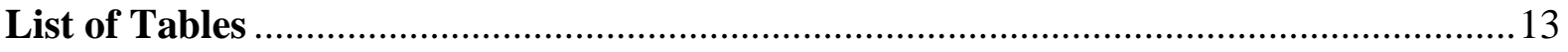

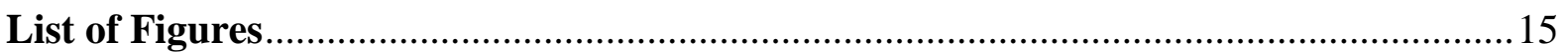

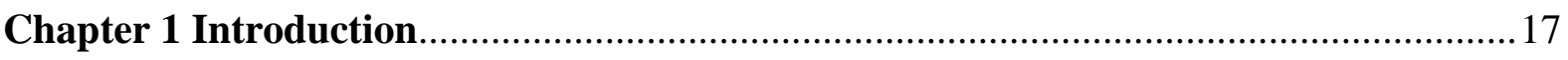

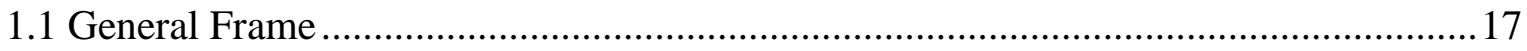

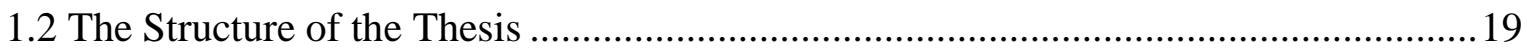

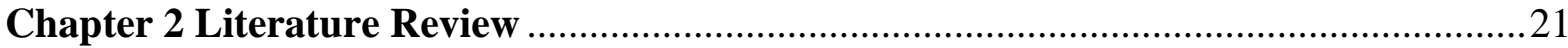

2.1 The Theoretical and Conceptual Framework of the Study ............................................21

2.2 Definitions and Main Concepts in the Self-Regulated Learning (SRL) Tradition .......23

2.3 Definition of Parental Support of Learning and Contingency ......................................26

2.4 Parental Supportive Behaviours, Children's Learning Skills and Performance ............31

2.4.1 Parental involvement in children's school learning, homework and education....31

2.4.2 Parental cognitive and metacognitive support, children's learning skills and

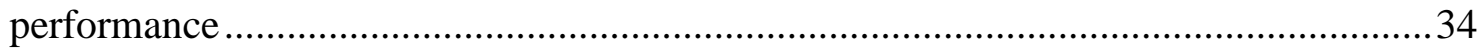

2.4.3 Parental emotional-motivational support and children's learning skills and performance.

2.4.4 Parental boosting the child's autonomy, parental control and children's learning skills and performance......................................................................................... 42

2.4.5 Studies exploring both cognitive-metacognitive and emotional-motivational

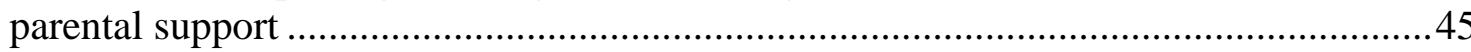

2.5 Factors Related to Parental Supportive Behaviours.........................................................4

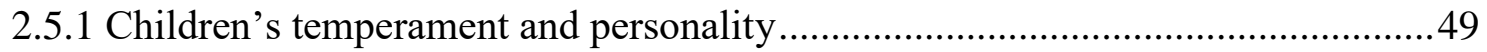

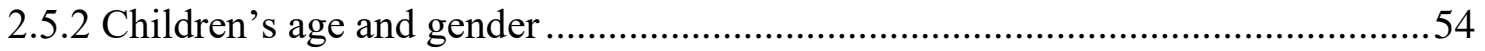

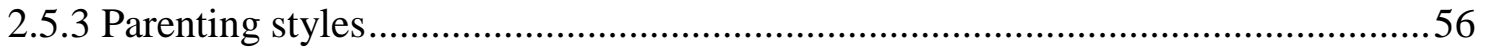

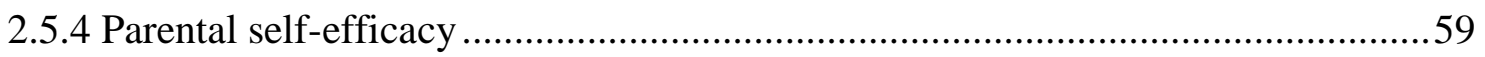

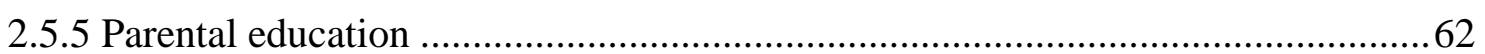

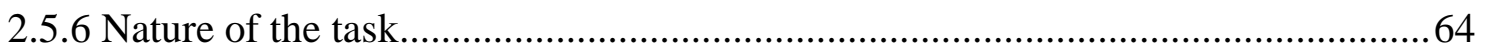

2.6 Factors Related to Children's SRL and Achievement …………………………...........67

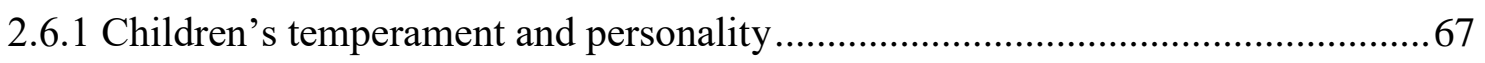

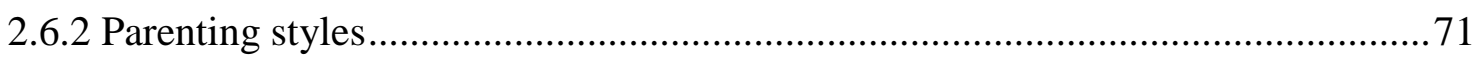

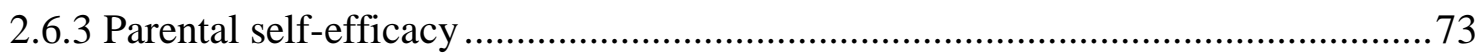

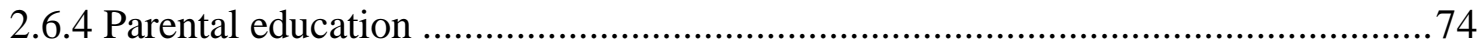

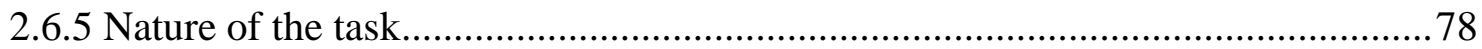

2.7 Rationale and Significance of the Study. Aims and Hypotheses of the Study .............80

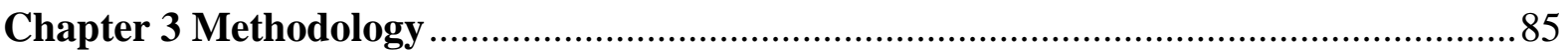

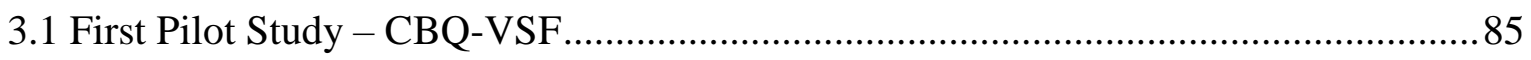

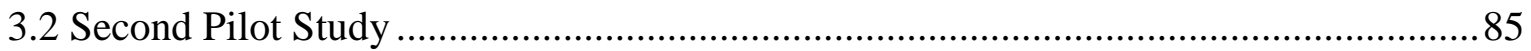

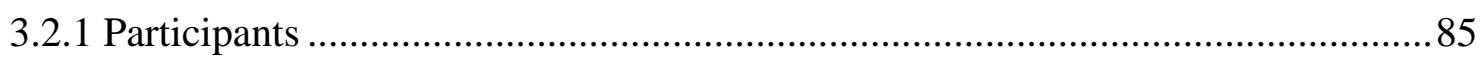


3.2.2 Measures. .86

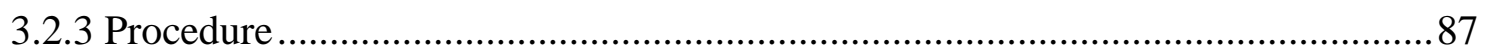

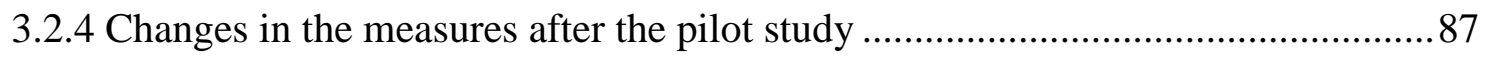

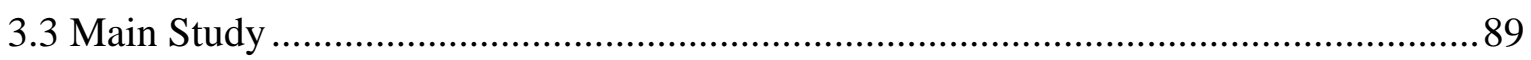

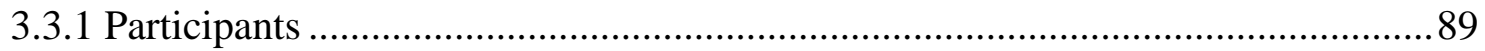

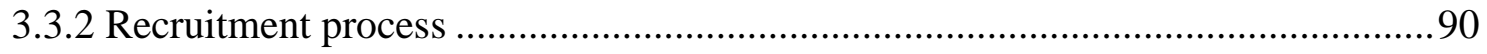

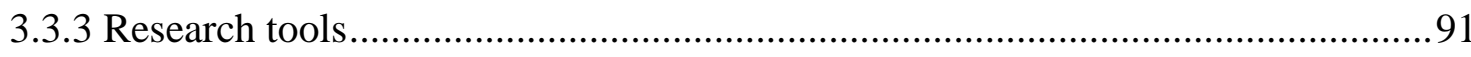

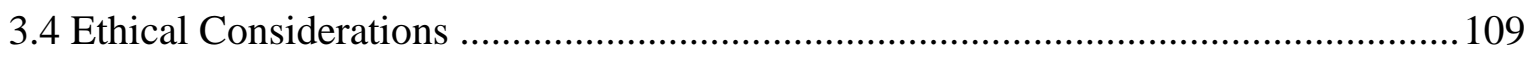

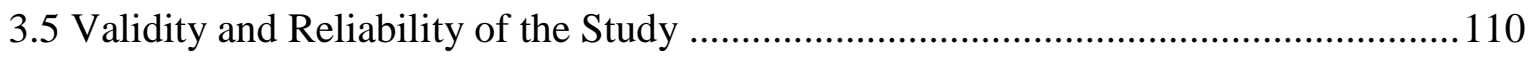

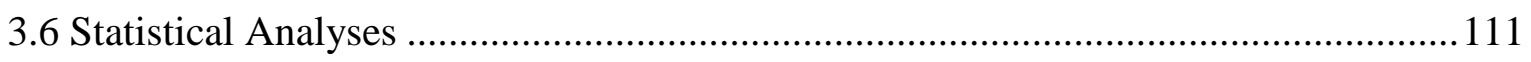

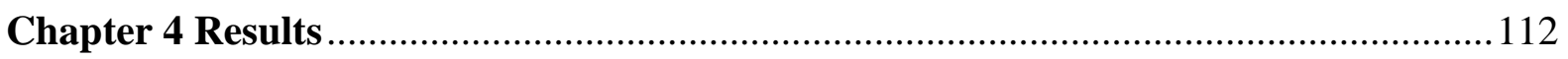

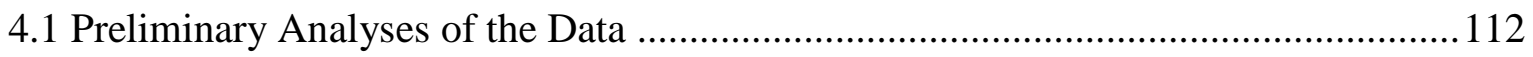

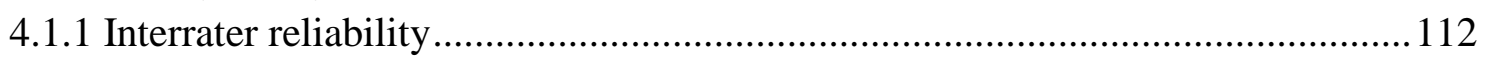

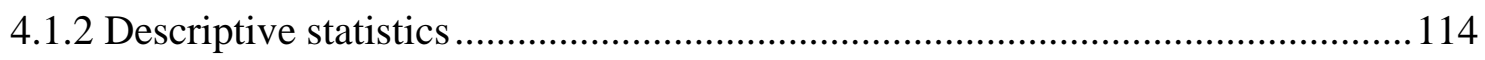

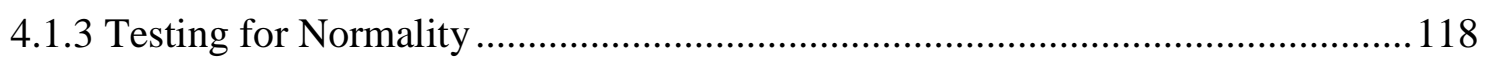

4.2 Differences in Maternal Measures and Differences in Children's Measures............... 120

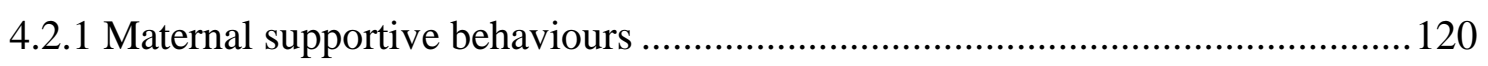

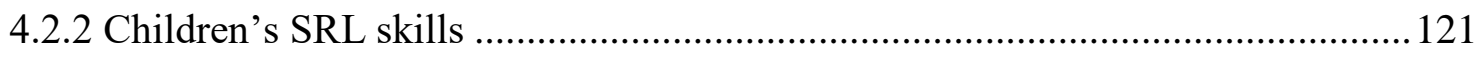

4.3 Children's and Mothers' Demographic Variables and Characteristics ........................123

4.3.1 Demographic variables: Associations with maternal supportive and contingent

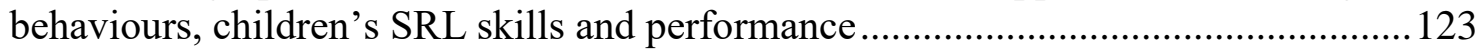

4.3.2 Maternal and children's characteristics: Associations with maternal supportive and contingent behaviours, children's SRL skills and performance ........................... 127

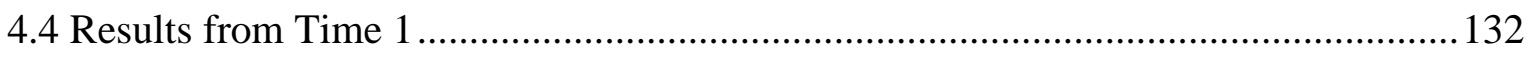

4.4.1 Maternal supportive and contingent behaviours and children's SRL skills and

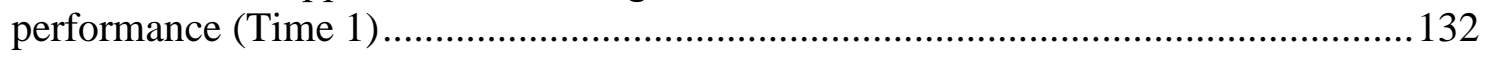

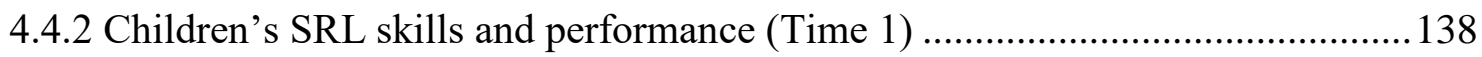

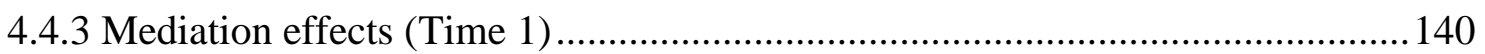

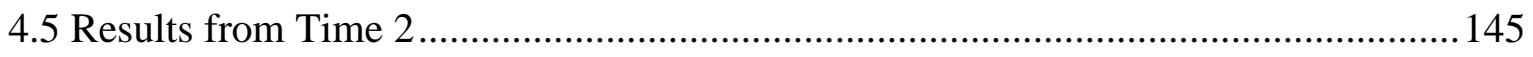

4.5.1 Maternal supportive and contingent behaviours and children's SRL skills and

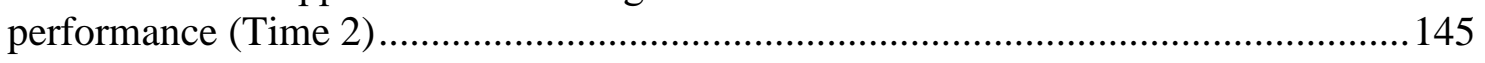

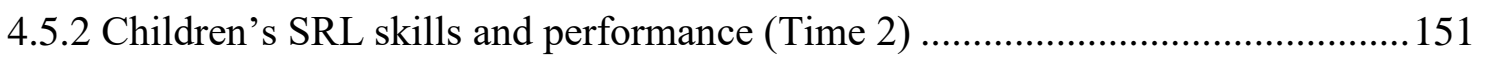

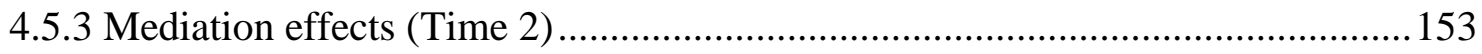

4.6 Longitudinal Findings: Results from Time 1 to Time 2 ......................................... 157

4.6.1 Early maternal supportive and contingent behaviours (T1) and children's later

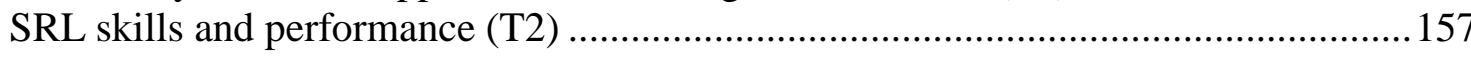

4.6.2 Children's early SRL skills (T1) and children's later SRL skills and performance (T2) 161

4.6.3 Mediation effects 166 
4.7 Longitudinal Observations in Maternal Supportive and Contingent Behaviours and in

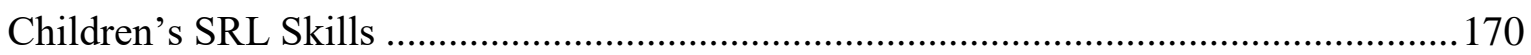

4.8 Differences in the Measures According to the Nature of the Task ............................. 171

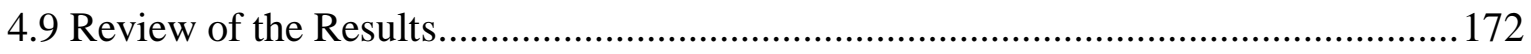

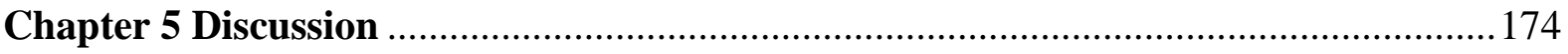

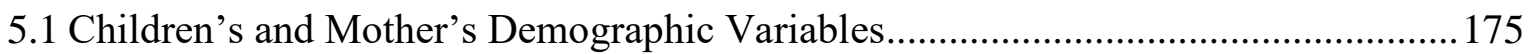

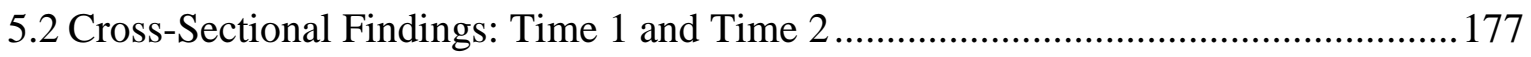

5.2.1 Relations between children's SRL skills and cognitive performance .................. 177

5.2.2 Maternal supportive and contingent behaviours and children's SRL skills and

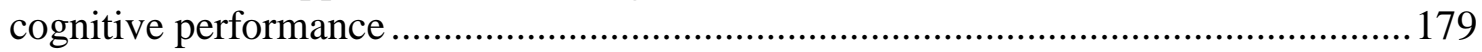

5.3 Children's and Mothers' Characteristics: Relations to Maternal Supportive and Contingent Behaviours and Children's SRL Skills and Cognitive Performance............... 182

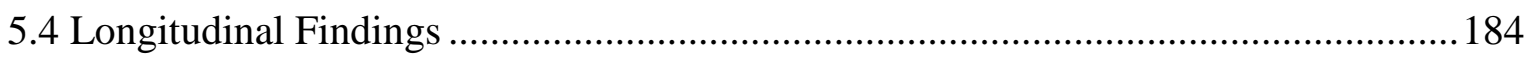

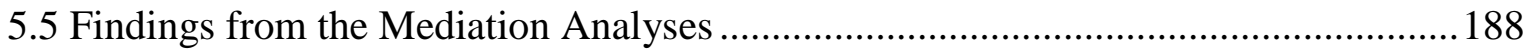

5.6 Short-Term Longitudinal Observations of Maternal Supportive and Contingent

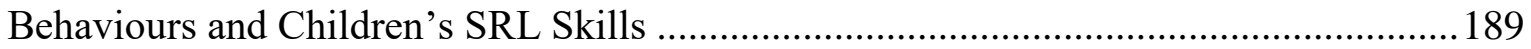

5.7 Differences in the Measures According to the Nature of the Task ............................. 191

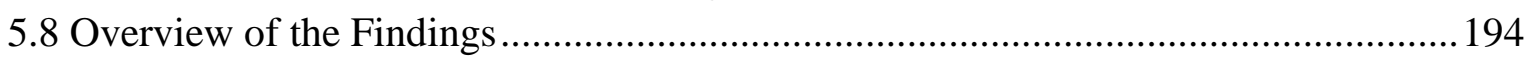

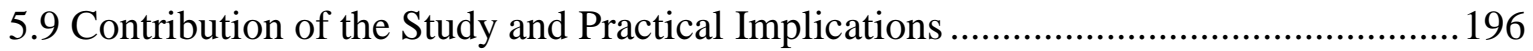

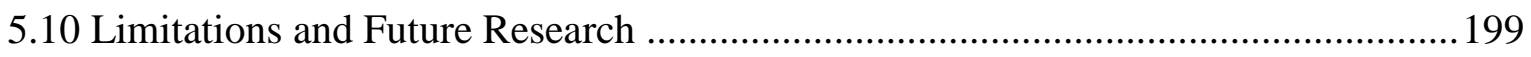

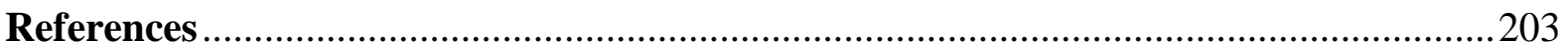




\section{List of Tables}

Number

Title

Page

Table 3.1 Changes in the Maternal Observation System After the Pilot Study

Table 3.2 Scoring Criteria for the Performance in the Language Tasks

Table 3.3 Examples of the Description and Scoring of Children's SRL Skills

100

Table 3.4 Example of Assessing Children's SRL Skills and Performance in the Visual-spatial Tasks

101

Table 3.5. Examples of the Description and Scoring Criteria for the Maternal Supportive Behaviours

Table 3.6 Example of Scoring Maternal Supportive Behaviours in the Visualspatial Tasks

Table 3.7 Maternal Contingency: Mother's Responsiveness to the Child's Understanding and Cognitive Needs

Table 4.1 Inter-rater Reliability for Maternal Supportive Behaviours - Time 1

Table 4.2 Inter-rater Reliability for Children's SRL Skills and Performance in the Language Tasks - Time 1

Table 4.3 Descriptive Statistics of the Measures

Table 4.4 Descriptive Statistics for Mothers' and Children's Scales (Time 1)

Table 4.5 Descriptive Statistics for Aggregate Scores of the Measures

Spearman's Correlations between Maternal Education, Maternal

Table 4.6 Supportive and Contingent Behaviours and Children's SRL Skills and Performance in the Visual-spatial and Language Tasks

Spearman's Correlations between Children's Age, Maternal

Table 4.7 Supportive and Contingent Behaviours and Children's SRL Skills and Performance in the Visual-spatial and Language Tasks

Table 4.8 Spearman's Correlations between Maternal and Children's Characteristics and Maternal Support in the Visual-spatial Tasks

Table 4.9 Spearman's Correlations between Maternal and Children's Characteristics and Maternal Support in the Language Tasks 
Spearman's Correlations between Maternal and Children's

Table 4.10 Characteristics and Children's SRL Skills and Performance in the Visual-spatial Tasks

Spearman's Correlations between Maternal and Children's Table 4.11 Characteristics and Children's SRL Skills and Performance in the Language Tasks

Partial Correlations between Maternal Dimensions and Children's Table 4.12 Skills and Performance in the Visual-spatial and Language Tasks (T1), Controlling for Children's Age

Table 4.13 Multiple Regression Analysis for the Visual-spatial Tasks: Predicting Children's SRL Skills and Performance from Maternal Support (T1)

Multiple Regression Analysis for the Language Task: Predicting Table 4.14 Children's SRL Skills and Performance from Maternal Support (T1)

Table 4.15 Partial Correlations between Children's SRL Skills and Performance Controlling for Children's Age (Time 1)

Multiple Regression Analysis for the Visual-spatial and Language

Table 4.16 Tasks: Predicting Children's Performance from Children's SRL Skills (T1)

Partial Correlations between Maternal Support and Children's Skills Table 4.17 and Performance in the Visual-spatial and Language Tasks (T2), Controlling for Children's Age

Table 4.18 Multiple Regression Analysis for the Visual-spatial Tasks: Predicting Children's SRL Skills and Performance from Maternal Support (T2)

Table 4.19 Multiple Regression Analysis for the Language Task: Predicting Children's SRL Skills and Performance from Maternal Support (T2)

Table 4.20 Partial Correlations between Children's SRL Skills and Performance Controlling for Children's Age (T2)

Multiple Regression Analysis for the Visual-spatial and Language Table 4.21 Tasks: Predicting Children's Performance from Children's SRL Skills (T2)

Partial Correlations between Maternal Support (T1) and Children's

Table 4.22 Skills and Performance in the Visual-spatial and Language Tasks (T2), Controlling for Children's Age

Multiple Regression Analysis for the Visual-spatial Tasks: Predicting Table 4.23 Children's SRL Skills and Performance (T2) from Maternal Support (T1) 
Partial Correlations between Children's SRL Skills (T1) and

Table 4.24 Children's SRL Skills (T2) and Performance (T2) Controlling for

Children's Age (T2)

Multiple Regression Analysis for the Visual-spatial Tasks: Predicting

Table 4.25 each of the Children's SRL Skills and Performance (Time 2) from Children's SRL Skills (T1)

Multiple Regression Analysis for the Language Task: Predicting each Table 4.26 of the Children's SRL Skills and Performance (Time 2) from Children's SRL Skills (T1)

\section{List of Figures}

Number

Title

Page

Parallel mediation model predicting children's Cognitive and

Figure 1 Metacognitive skills from children's age, maternal Contingency and maternal Autonomy support in the Visual-spatial tasks T1

Mediation model predicting children's Emotional and Motivational

Figure 2 skills from children's age and maternal Autonomy support in the Visual-spatial tasks T1

Mediation model predicting children's Cognitive and Metacognitive

Figure 3 skills from children's age and maternal Contingency in the Language tasks T1

Mediation model predicting children's performance from children's age and maternal Contingency in the Language tasks T1

Mediation model predicting children's performance from maternal

Figure 5 Autonomy support through children's Cognitive and Metacognitive skills in the Language tasks T1

Mediation model predicting children's performance from maternal

Figure 6 Autonomy support and children's Cognitive and Metacognitive skills in the Visual-spatial tasks T2

Mediation model predicting children's performance from maternal

Figure 7 Contingency and children's Cognitive and Metacognitive skills in the Visual-spatial tasks T2

Mediation model predicting children's performance from maternal

Figure 8 Autonomy support and children's Cognitive and Metacognitive skills in the Language task $\mathrm{T} 2$ 
Parallel mediation model predicting children's Cognitive and

Figure 9 Metacognitive skills (T2) from maternal Autonomy support (T1) and children's SRL skills (T1) in the Visual-spatial tasks

Parallel mediation model predicting children's performance (T2)

Figure 10 from maternal Contingency (T1) and children's Cognitive and 168 Metacognitive skills (T1 and T2) in the Visual-spatial tasks

Mediation model predicting children's Cognitive and Metacognitive

Figure 11 skills (T2) from maternal Contingency (T1) and children's Cognitive and Metacognitive skills (T1) in the Visual-spatial tasks

Serial mediation model predicting children's performance (T2) from

Figure 12 maternal Contingency and children's Cognitive and Metacognitive 169 skills (T1 and T2) in the Visual-spatial tasks 


\section{Chapter 1 Introduction}

\subsection{General Frame}

Over the past century, significant research emerged from a sociocultural and a sociocognitive point of view regarding children's learning and the impact of the environment on promoting children's skills. From 1980 and beyond, researchers have started focusing their interest on examining children's problem-solving in specific contexts, including adult-child interactions. Previously, Vygotsky (1978) had underlined that children's learning evolves through children's interaction with their environment, and in particular, with more competent individuals. More recently, growing attention aroused around specific parental practices, such as positive parental affect or cognitive demands. In this respect, studies have attempted to identify the way parents could support their children's learning by examining different aspects of parental support.

According to Zimmerman (2008) "Self-Regulated Learning is viewed as proactive processes that students use to acquire academic skill, such as setting goals, selecting and deploying strategies, and self-monitoring one's effectiveness, rather than as a reactive event that happens to students due to impersonal forces" (pp. 166-167). These skills are positively linked to cognitive and academic benefits (Zimmerman, 2002). Children's SRL skills start developing during the early preschool years (Bronson, 2000). The ever-increasing findings that highlight the critical role of SRL skills in different academic outcomes have made this subject an essential aspect of contemporary investigations. Central to the entire field of SRL is how adults can support children in the development of these skills. Therefore, a considerable amount of research has evolved regarding the way teachers and parents or even classmates can help students by taking on an associate, active role.

In the context of family, Wood, Bruner, and Ross (1976) referred to "parental scaffolding" to describe successful parental supportive behaviours for children's developing skills. Recent trends on this matter have led studies to the examination of various aspects of parental support and their impact on children's learning (Johnson, Cowan, \& Cowan, 1999; Stright, Neitzel, \& Herr, 2009). These parenting behaviours were examined mostly during parent-child interactions.

This study investigates possible connections between parental support of learning and children's self-regulation of learning. This connection is guided by bridging three learning theories, the Social Cognitive Theory, the Socio-Cultural Theory and SRL from a socio- 
cognitive point of view. Schunk and Zimmerman (1997) reviewed relevant research on the social origins of students' self-regulatory competence. In this respect, the social-cognitive view of self-regulation considers social models as a significant basis for communicating selfregulatory skills to children and for the development of children's self-efficacy. Selfregulatory competence builds up from the social sources and consequently moves to selfsources. From a sociocultural view, social interactions between adults or mature peers and children assist in the development of self-regulatory skills. Children's self-directed speech, which is viewed as the beginning of self-regulatory control, is assisted by the adult-child "reciprocal teaching" and "social dialogue" (Schunk \& Zimmerman, 1997, p. 200).

The current investigation adopts a microanalytic and longitudinal methodology to explore the relations between different aspects of maternal supportive and contingent behaviours activated during parent-child joint problem-solving and children's SRL skills and performance. In particular, these relations are examined both at one specific time point (cross-sectionally) and longitudinally. The maternal supportive behaviours and children's SRL skills are observed at a micro-level with a focus on actual parent-child interactions episodes at two different time points with a six-month interval between them. This study also takes into account the unique characteristics of the participant mothers and children, such as maternal education, children's age and gender, children's temperament, maternal selfefficacy and parenting style, as these factors have been previously found to be associated with the variables under examination.

This thesis attempts to provide further insights into mother-child interactions during problem-solving, a research area that, so far, is given too little attention. There are limited studies both internationally and in Greece on the above issues, and the existing literature to date has given more emphasis to parental intervention in homework settings and in schoolaged children. Regarding the greek context, the limited number of studies report that Greek parents are involved in children's educational activities taking place at home (e.g., homework, book reading) (Kafoussi, 2009; Natsiopoulou, Souliotis, Kyridis, \& Hatzisavvides, 2000). Petrogiannis (2013) provided some insights about preschool education and parental involvement in the Greek context. Although nowadays significant reforms are taken place regarding the time children should begin a compulsory education, until recently, formal education began at five years old, when children were expected to attend one year of kindergarten before entering to elementary school. At the moment, two years of compulsory pre-school education has been voted and is about to be gradually employed in some regions of the country. Nurseries and kindergartens either private or state provide early childhood 
education. It is reported that parents of very young children seem not to be so much involved in their children's nursery's activities and other educational issues, but they are more concerned for safety or nutrition matters (see, for a review, Petrogiannis, 2013). However, they do hold expectations for the education services.

The present study will focus on mothers and their pre-school children who all attended kindergarten or pre-kindergarten classes. As this period is critical for children's developing skills, it is important to understand whether and how maternal behaviours contribute to this development. Research in the domain of mother-child early problemsolving interactions might provide valuable insights into pre-school education and the significant role of parents in promoting children's learning.

\subsection{The Structure of the Thesis}

Overall, this dissertation has five chapters. The first is the present one in which the general content of the thesis is given, and the broader areas of research are introduced. The second chapter consists of seven sections and in general, addresses the theoretical dimensions of the research. The first section presents the theoretical and conceptual framework of the study. The second section refers to the definitions and the main concepts in the SelfRegulated Learning Tradition. The third section provides the definitions of the parental support of learning and parental contingency. The fourth section provides a review of the literature regarding the links between the parental supportive behaviours and children's learning skills highlighting the need for further studies in this field, especially from a multidimensional perspective. The fifth and sixth sections deal with the investigation of the factors that have been found to be related with the central concepts of this research, such as demographic variables, children's temperament, maternal self-efficacy, parenting styles and the cognitive domain. The last section introduces in detail the study's research aims and the research hypotheses.

The third chapter considers the methodology used to test the research hypotheses. The chapter is being structured in six sections, including the detailed description of the pilot and the main study, information about the participants, the procedure, the measures and the tasks, the assessment and scoring criteria as well as some ethical, validity and reliability issues.

The fourth chapter is concerned with the results of the study and presents the findings of the statistical analyses in nine sections. The relations between children's and mothers' demographic variables and characteristics with the main variables of the research are 
examined. Moreover, the cross-sectional and short-term longitudinal findings of the links between the maternal supportive and contingent behaviours with children's SRL skills and performance are presented.

In the fifth chapter of this thesis, the findings of the study are discussed, comparing the results with previous literature data. It also includes some general conclusions that highlight the importance of the results for interventions addressed to both parents and teachers. Finally, the chapter ends with the study's limitations and some future research suggestions. 


\section{Chapter 2 Literature Review}

\subsection{The Theoretical and Conceptual Framework of the Study}

Previous research examining how adults can influence children's learning have shown an increased interest in one particular concept, that of "scaffolding". The term generally refers to the support or help yielded by an adult or more capable peer to facilitate children's skills and knowledge. This term also encompasses a form of "guidance" which is provided by the child's environment within the Zone of Proximal Development (Vygotsky, 1978); this interaction is based on instructions from a more experienced teacher or adult to a student during problem-solving (Wood, Bruner, \& Ross, 1976; Wood \& Middleton, 1975).

The following paragraphs provide a summary of the fundamental learning theories used by this dissertation as a theoretical and conceptual framework.

The Social Cognitive Theory underlines the significant role of the social environment and its learning conditions for children's learning. According to Bandura (1983), human functioning involves an exchanged interplay between personal variables, behaviours and environmental factors (referred to as triadic reciprocality). The theory supports that children can obtain knowledge, skills and strategies through the observation of other individuals (Schunk, 2012). "The social learning analysis of observational learning (Bandura, 1971) assumes that modeling influences operate principally through their informative function, and that observers acquire mainly symbolic representations of modeled events rather than specific stimulus-response associations" (Bandura \& Jeffery, 1973, p. 122). As Schunk (2012, p.121) mentions, "Learning occurs either enactively through actual doing or vicariously by observing models perform (e.g., live, symbolic, portrayed, electronically)".

The Social Cognitive Theory also supports that individuals can self-regulate their emotions, behaviours, cognitions and environments to achieve their goals. In broad terms, children, in their effort to acquire new skills, observe other individuals (models) analyse and demonstrate these skills to children. Cognitive growth is affected by both maturation and "exploratory experiences", although the most beneficial information is socially communicated (Bandura, 1989). In addition, significant others play an important role in providing knowledge that forges the way children think and their cognitive development through guided instructions and modelling. This type of learning that is socially-driven promotes children's self-directed learning as children have access to the appropriate conceptual tools required to understand and acquire new knowledge and information about 
how to act in new circumstances. As individuals grow up, they rely more on their own experiences and inquire help when they feel uncertain.

The Sociocultural Theory considers "human learning and development as intrinsically social and interactive", and underlines the complex interactions between individuals and their social environment, confronting the position that these two can be viewed as separate factors (Street, 2004, p.8).

From 1980 and beyond, researchers have started focusing on adult-child interactions to examine children's problem-solving in a social context (e.g., Rogoff, Ellis, \& Gardner, 1984; Wertsch, McNamee, McLane, \& Budwig, 1980). Vygotsky (1978) had already stressed the importance of children's interaction with more competent peers or adults for the development of children's learning skills. This joint experience between children and more skilled individuals (e.g., parents) is an essential factor that fosters children's cognitive abilities (Mulvaney, McCartney, Bub, \& Marshall, 2006).

The most popular concept of Vygotsky's theory is the term "Zone of Proximal Development" (ZPD). ZPD was defined as "the distance between the actual developmental level as determined by independent problem-solving and the level of potential development as determined through problem-solving under adult guidance or in collaboration with more capable peers" (Vygotsky, 1978, p.33). This definition can be loosely explained by viewing this term as the discrepancy noticed between what children can achieve based on their skills and what they could accomplish with the support from others. Elucidating more this concept, the Zone of Proximal Development involves a "reciprocal nature of interaction", an interaction between a more capable person and a less capable individual in a problem-solving situation, in such a way that both the adult and the child participate in learning and teaching (Chaiklin, 2003). Cognitive change in the ZPD could appear during the sharing of cultural tools between teachers and students and "when it is internalized in the learner" (Bruning, Schraw, Norby, \& Ronning, 2004; Cobb, 1994, as both cited in Schunk, 2012, p. 244).

As Mulvaney et al. (2006) mentioned, Vygotsky's theory explained how children could develop their cognitive skills through their interpersonal interactions. According to this position, skills related to culture and higher-order cognitive skills (e.g., planning and reasoning) could be encouraged by well-structured interactions. During this mutual time, the more capable individuals share with the novices the knowledge and understanding of the task, problem, concept or strategy while they are both engaged in a "coconstructed, dialectical process". Parents, teachers and capable peers when interacting with the child provide advantageous instruction within the child's Zone of Proximal Development. For example, 
teachers use different strategies to support children's learning and adjust this scaffolding to their student's needs in order for the child to complete a task or understand a new concept or strategy and be able to use this new knowledge to similar tasks in the future (Chatzistamatiou \& Dermitzaki, 2013; Hammond \& Gibbons, 2005). When children's learning takes place in an environment which is high in challenge and support, then students benefit the most (Mariani, 1997).

\subsection{Definitions and Main Concepts in the Self-Regulated Learning (SRL) Tradition}

An extensive amount of literature has highlighted the significance of self-regulated learning skills in students' school and academic life (e.g., Dignath, Buettner, \& Langfeldt, 2008; Järvelä, Järvenoja, \& Malmberg, 2012; Trainin \& Swanson, 2005; Zimmerman, 2008). Bandura's socio-cognitive theory regards individuals as "proactive and self-regulating" operators who are not passively affected by their environment (Pajares \& Valiante, 2002, p. 211). According to Zimmerman (2000, p. 14) SRL "refers to self-generated thoughts, feelings, and actions that are planned and cyclically adapted to the attainment of personal goals". Although there is not one simple definition (Boekaerts \& Corno, 2005), there are some basic components of SRL that are critical for classroom performance, such as cognitive strategies, metacognitive strategies, management and control of the effort (Pintrich \& De Groot, 1990). Paris and Paris (2001) stated that "it is the fusing of skill and will (and dare we add "thrill?") to emphasise that cognition, motivation, and affect are all involved in selfregulated learning" (p. 91). Butler (2011) noted that "over time a consensus has emerged about the nature of SRL that suggests important parameters for its study. Specifically, it is widely agreed that SRL is a multi-componential, dynamic, recursive, contextualised activity that constitutes both individual and social processes" (p. 351). Previously, Pintrich (2000) proposed that SRL "is an active, constructive process whereby learners set goals for their learning and then attempt to monitor, regulate, and control their cognition, motivation, and behaviour, guided and constrained by their goals and the contextual features in their environment" (p. 453). These self-regulatory activities can mediate the relationships between individuals and the context, and their overall achievement. Pintrich (1995) had also stressed the importance of self-reflection, positive motivational beliefs and practice in children's efforts to self-regulate their learning.

SRL theories seek to portray the way that the learning process is affected by cognitive, motivational and contextual determinants. Among the prominent models of SRL is 
the cyclic model of Zimmerman $(1998,2002)$ in which three phases are described in each cycle. "The forethought phase refers to processes and beliefs that occur before efforts to learn; the performance phase refers to processes that occur during behavioural implementation; and the self-reflection phase refers to processes that occur after each learning effort" (Zimmerman, 2002, p. 67). Pintrich (2000) described four phases and areas for regulation, consisting of the Forethought phase, the Monitoring Phase, the Control phase and the Reaction/Reflection phase. Winne and Hadwin (1998) presented a model of SRL, which included four phases; the development of a model of the task, goal setting - planning studying tactics and strategies, the application of the tactics and strategies, and adaptations to metacognition. Five facets are included in each phase: conditions, operations, products, evaluations and standards (COPES model) (Winne, 2004). Boekaerts (1999), in her article "Self-regulated learning: Where we are today", presented the three-layered model of SRL outlining three processes, namely Regulation of the self, Regulation of the learning process and Regulation of processing modes. A more recent approach of SRL is Efklides' (2011) MASRL Model which presents the Metacognitive and Affective Model of Self-regulated Learning (MASRL) illustrating interactions between metacognition, motivation and affect in SRL.

Self-regulated learning strategies. SRL skills are strongly linked with significant cognitive benefits in academic settings (Boekaerts, 1999; Borkowski, Chan, \& Muthukrishna, 2000; Zimmerman, 2002). Previous studies have supported that children who demonstrate higher SRL skills learn more efficiently, are better motivated towards learning, show better adaptation to school and better academic performance (Dermitzaki, Andreou, \& Paraskeva, 2008; Efklides, 2011; Rimm-Kaufman, Curby, Grimm, Nathanson, \& Brock, 2009; Zimmerman \& Schunk, 2008).

In particular, children who self-regulate their learning process seem to actively engage in learning using metacognitive, behavioural and motivational strategies (Zimmerman, 2002). In general, these students are acquainted with different strategies, and they know how to implement these strategies in order to process and organize the information given; they are also capable of planning and controlling their cognitive processes with a view to achieving a goal, they show high levels of self-efficacy, they approach a task with a positive manner, they possess time and effort management techniques, they shape their environment according to their needs, they activate suitable strategies to remain concentrated and motivated to the task and they can isolate possible distractions (Weinstein, Husman, \& Dierking, 2000; Zimmerman, 2002). 
Although SRL is not always apparent in very young children, its development begins early and becomes critical as children grow up and are expected to take responsibility for their learning (Bronson, 2000; Dermitzaki \& Kiosseoglou, 2004). For example, studies have reported the development of both executive function skills (e.g., focused attention, working memory) in preschool children (e.g., Kannass, Oakes, \& Shaddy, 2006; Wiebe et al., 2011; Bull, Espy, \& Senn, 2004) and self-regulatory behaviours, like monitoring and control (e.g., Whitebread et al., 2005; Whitebread, Bingham, Grau, Pino-Pasternak, \& Sangster, 2007). Person factors, such as students' age, goal orientation and self-efficacy (e.g., Chatzistamatiou, Dermitzaki, Efklides, \& Leondari, 2015), and environmental factors, such as the significant others, have been found to affect the development and use of SRL strategies in children.

This dissertation investigates cognitive, metacognitive and motivational/volitional aspects of SRL, based on the methodology proposed by Dermitzaki, Leondari, and Goudas (2009). Specifically, cognitive strategies include rehearsal, elaboration and organisational strategies that support students' learning and understanding (Boekaerts, 1996; Pintrich \& De Groot, 1990; Winne, 1996). Boekaerts (1996) defined cognitive strategies as the "cognitive processes and behaviours that students use during actual learning experiences to complete an assignment or to accomplish a goal implied by the academic task" (pp. 105-106). Cognitive strategies assist students in learning, remembering and comprehending the elements of a task (Frankel, 2016; Pintrich, 2004) and also in activating working memory, attention and encoding processes, making connections, combining and selecting information to previous knowledge (Pintrich, Smith, Duncan, \& Mckeachie, 1991).

Metacognitive strategies refer to planning, monitoring and changing one's cognition as well as to the control and evaluation of actions (Corno, 1986; Zimmerman \& MartinezPons, 1988). "Metacognitive skills is procedural knowledge" (Efklides, 2006, p. 5), that is "knowledge about doing things" (Schraw, 1998, p. 114), and it refers to "what the person deliberately does to control cognition" (Efklides, 2006, p. 5). Metacognitive skills assist learners to "plan, organise, self-instruct and self-evaluate" during different phases of the solution of a task (Zimmerman \& Martinez-Pons, 1988, p. 284). Nonetheless, students' performance and academic achievement do not depend only on cognitive and metacognitive strategies. Students must also be driven and prompted to employ these strategies (Pintrich \& De Groot, 1990).

Motivational and volitional strategies (e.g., goal oriented self-talk, interest enhancement) help the learner to initiate action and uphold or even boost his/her motivation 
towards the task and include behaviours, such as engagement to the task and demonstrating persistence in front of challenges (Mayer, 1998; Wolters, 2003). Students' motivation could vary during the process of solving a task. Thus, it is essential for students' learning to be able to maintain or enhance their willingness when dealing with or trying to accomplish an academic task (Schunk \& Zimmerman, 2008; Wolters, 1999). Volitional strategies, such as emotion regulation, attentional control and motivational control assist students in determining that their goals will be attained and help them to demonstrate higher persistence for academic tasks (Wolters, 1999).

Considering all the above, the importance of SRL skills for students' learning and academic achievement is well established. However, far too little attention has been paid to young children's strategies to regulate their learning. The research to date has tended to focus on school-aged students rather than on preschool children. However, there is some evidence for SRL in preschool years (Schneider, 2008); Some researchers have provided data on young children's efforts to regulate their learning (e.g., Bronson, 2000; Zhang \& Whitebread, 2017). Therefore, further research is needed to enhance our understanding of SRL in preschool age, a critical period in which these skills are developing. Moreover, new methodological approaches are also required when investigating SRL in students. Previous studies in the field relied mostly on teachers' and parents' questionnaires or students' self-reports to assess SRL. Such approaches, however, do not capture SRL skills in situations when students actually employ these skills during problem-solving contexts. Hence, new observational data during actual-online episodes are much needed, providing a more naturalistic approach of SRL. Longitudinal data should also be provided examining children's SRL skills over time. This thesis will attempt to address the above limitations by examining SRL in young children and during on-line problem-solving, providing valuable information for a better understanding of SRL in preschool children and in problem-solving contexts.

\subsection{Definition of Parental Support of Learning and Contingency}

Parental supportive behaviours. Before examining the definitions of parental support of learning, it is necessary to clarify how this thesis defines parental supportive behaviours and how this definition relates to those provided traditionally in the literature. The present study uses the term "parental supportive behaviours" to refer to specific parental behaviours or practices that are activated spontaneously during parent-child joint learning and problem-solving. These parental behaviours tap different aspects of parental support, such as 
cognitive and metacognitive support, emotional-motivational support and autonomy support. Related to the above term is the concept of "scaffolding" that is defined as "an instructional interaction that is aimed at extending the child's knowledge, reducing task complexity, and transferring responsibility while providing emotional support" (Bruner, 1986, as cited in Neitzel \& Stright, 2003, p. 147). In general, the term "scaffolding" has been widely used in studies; Pea (2004) pointed out that "the concept of scaffolding has become so broad in its meaning in the field of educational research and the learning sciences that it has become unclear in its significance" (p. 423), while Puntambekar and Hübscher (2005) commented that "the scaffolding construct is increasingly being used synonymously with support" (p. 1). Therefore, in the following paragraphs, a brief review of the literature of parental scaffolding and parental contingency will be given as these concepts were the foundation of this thesis' conceptualization of parental support of learning.

According to Puntambekar and Hübscher (2005), it is important to know that scaffolding does not constitute an official element of Vygotsky's theory; the term was formally introduced by Wood, Bruner, and Ross (1976). However, its general concept comes from Vygotsky's theory highlighting the significance of providing instructions that precede children's ongoing skills (Vygotsky, 1978). Wood et al. (1976) mentioned that an intervention of a tutor includes a "scaffolding process that enables a child or novice to solve a problem, carry out a task or achieve a goal which would be beyond his unassisted efforts" (p. 90). In this context, the adult's role is to take control of the more challenging aspects of the task (which cannot be solved by the learner) allowing the learner to deal with those issues that are within his/her scope of skills. Wood et al. (1976) described six sub-processes of scaffolding: 1. Recruitment (e.g., attracting the learner's interest), 2. Reduction in degrees of freedom (e.g., making the task easier by decreasing the steps to achieve the goal), 3. Direction maintenance (e.g., keeping the child engaged in the task, emotional support, etc.), 4. Marking critical features (e.g., highlighting relevant characteristics of the task), 5. Frustration control (e.g., the presence of the tutor makes the task less stressful), and 6 . Demonstration (e.g., modelling strategies or solutions).

For optimal scaffolding, children must be fully engaged in the task solution process, and, even better, both the child and the adult should maintain their concentration on the project. In this context, Martin and Reutzel (1996) found that mothers used different strategies, according to their children's age, to keep their children's attention. Toddlers needed more simple strategies, such as shared control, while preschool children needed other types of engagement strategies, such as the dialogue. Finally, children should resume more 
control as they acquire more skills and become more capable of handling the task (Conner \& Cross, 2003).

It should be noticed that throughout this thesis, the term "parental support of learning" will be used to refer to the different aspects of parental behaviours activated to support children's learning when needed. However, as this term comes from the scaffolding literature, the term "scaffolding" will also be mentioned in the text, especially when it is employed by the researchers of a study so that to be consistent with each author's terms and definitions.

Types of parental support of learning. To our knowledge, only a few studies that explored parental-child interactions during learning have studied simultaneously different dimensions of parental support, such as contingency, cognitive and metacognitive support, emotional-motivational and children's autonomy support. The relevant literature, however, is rich. Indeed, some studies have investigated different aspects of parental supportive behaviours and their relation to children's learning skills, executive function or academic performance, providing valuable information on the subject. For example, Robinson, Burns, and Davis (2009), based on the work of Kermani and Brenner (2000), examined different aspects of maternal scaffolding that was defined as "any verbal or physical action that was intended to provide guidance or assistance to the child in the parent-child puzzle-matching task" (p. 89). In their study, the researchers included six parental behaviours: encouragement of independence, explanation, strategic question, verbal hint, verbal prompt and praise. The study of Salonen, Lepola, and Vauras (2007) used both global ratings and micro-analytic approaches for measuring parent-child behaviours. Regarding the global ratings, the examiners' assessment took into account "parental socio-cognitive and socio-emotional scaffolding match vs mismatch", defined as the ability of the parents to effectively adapt to the changes in children's independent functioning and emotional responses. The microanalytic coding included behaviours such as intrusiveness, directiveness, socio-emotional responses and their dyadic co-ordinations and verbalisations of expectations/attributions anticipating success or failure. Neitzel and Stright (2003) coded maternal behaviours using three aspects of parental support, that is, cognitive support, emotional support and transfer of responsibility. Stright et al. (2009) focused on maternal cognitive support, directiveness, praise and criticism. Mattanah et al. (2005) assessed parental responsiveness, demandingness, autonomy support and contingent scaffolding. The work of Pino-Pasternak, Whitebread, and Tolmie (2010) included multidimensional measures of parent-child interaction, such as 
socioemotional behaviours (e.g., affect, responsiveness and control), parental instructional behaviours and parental contingency.

For the purposes of this study, the assessment of parental support follows the work of Neitzel and Stright (2003, 2004). Therefore, three main aspects of parental supportive behaviours will be examined: parental cognitive and metacognitive support, emotionalmotivational support and autonomy support.

Parents' cognitive and metacognitive support during problem-solving refers to the instructions given to children in an appropriate way and a developmentally suitable manner to lighten the cognitive load of the children (Wood et al., 1976, as cited in Stright et al., 2009). According to Neitzel and Stright (2004), "Parents may provide cognitive support by reducing the cognitive demands of the task for their children; providing instructions in discrete steps and extending their children's understanding of the task by sharing metacognitive information" (p. 166). In Neitzel and Stright's work (2003, 2004), parental cognitive support includes two aspects; first, the provision of metacognitive information which refers to any strategy given to the child about ways of managing the task or to further develop the child's understanding, and second, the manner of instruction which incorporates the regulation of the task's difficulty and the review of the progress.

Parents' emotional-motivational support is considered fundamental to the development of children's intrinsic motivation and personal competence (Ginsburg \& Bronstein, 1993). Parental emotional support could be provided using verbal or non-verbal praise and encouragement or conversely, by criticising or rejecting the child's efforts (Neitzel \& Stright, 2004). Even though studies have used different conceptualisations, the most common terms referring to parental emotional support is parental warmth, positive affect, supportive presence, responsiveness, encouragement or sensitivity (e.g., Berlin, BrooksGunn, Spiker, \& Zaslow, 1995; Landry, Smith, \& Swank, 2006; Pino-Pasternak, Whitebread, $\&$ Tolmie, 2010), which indicate a general demonstration of positive feeling from the parent to the child.

Finally, parents' support of children's autonomy or transfer of responsibility refers to parents' ability to gradually hand over the solution process to their children (Neitzel \& Stright, 2003). This shifting requires from parents to provide fewer instructions, permitting children to take on more responsibilities regarding the solution of the task. In other words, the meaning of parental support lies in this transferring of responsibility to the children, as they become increasingly more skilled and competent. Therefore, parents must be able to understand and determine when is the right time for their children to undertake this role, by 
providing assistance when it is needed or by restraining their instructions respectively. Neitzel and Stright (2003) recognised two aspects of autonomy support (or transfer of responsibility, as it was used in their work), that is parental overcontrolling and parental encouragement of active involvement. Parental overcontrolling behaviours point out the parents' attempts to control the child's action while the child shows evidence that $\mathrm{s} /$ he can handle the task while parental encouragement of active involvement refers to parents' attempts to boost the child's cognitive and active role in the problem-solving process by using prompts and questions.

Parental contingency. One more critical concept expressed by Wood and Middleton (1975) is the "region of sensitivity"; the term refers to the adult's acknowledgement of what parts of the task could be independently managed by the child, what parts could be understood by the child but not completed, and what parts the child neither could understand nor perform. Wood and Middleton (1975), examining mother-child interaction during problem-solving, noticed that when children came across difficulties during the solution process, mothers tended to provide more instructions, while the opposite was observed when children were successful at handling the task independently. This pattern of interaction is mentioned as the "contingent shift principle" and includes two complementary requirements, that of supporting the child when confronted with difficulties or failure and that of providing less assistance when the child seems to manage the task efficiently. Along similar lines, Pratt, Green, MacVicar, and Bountrogianni (1992) found that parents provided more assistance to their children in the difficult tasks and to those children who had more difficulties. Both the above studies showed that mothers who employed more the contingent shift principle had children who demonstrated greater skills. In other words, it seems that the extent to which parental scaffolding positively influences children's skills and performance is associated with the contingency with which this support is provided. Accordingly, parents not only have to be challenging but they also have to modify their level of scaffolding to correspond with children's level of understanding or mastery of the task (Pino-Pasternak, Whitebread, \& Tolmie, 2010).

In summary, it could be purported that parental scaffolding refers to the support and assistance offered by parents in the difficult points of the task that is beyond children's range of skills, while at the same time parents let children deal with the parts that are within their ability range. Contingency refers to parents' ability to adequately provide assistance when actually needed or to properly remain uninvolved based on signs of children's ongoing understanding and mastery of the task. 


\subsection{Parental Supportive Behaviours, Children's Learning Skills and Performance}

The literature so far suggests that parent-child interactions during problem-solving or general play activities have a significant impact on children's development of skills and knowledge. Children could benefit from this support and interaction and could gradually develop cognitive, behavioural, social and emotional skills. It has been shown that early parental behaviours (e.g., cognitive assistance) may be positively related to children's later skills and performance in problem-solving tasks (e.g., Fagot \& Gauvain, 1997; Landry, Miller-Loncar, Smith, \& Swank, 2002). The positive contribution of parental supportive behaviours during parent-child interactions is also apparent in children's social skills (Leve \& Fagot, 1997).

This thesis will focus on parental support during joint problem-solving and in particular, on parental supportive behaviours that are hypothesised to affect the development of children's learning skills. Therefore, in the following sections, the literature review will examine the relations between different parental supportive behaviours, children's learning skills and performance.

\subsubsection{Parental involvement in children's school learning, homework and education}

It is supported that parents are involved in their children's education for three main reasons; they believe that their role as parents inludes their involvement in their children's education, "they have a sense of personal efficacy" and they believe that they could help their children in school, and finally, they believe that the school or their children call for or wish their participation (Hoover-Dempsey \& Sandler, 1995, pp. 313-315). In general, parental involvement refers to the behaviours adopted by parents both at home and at school to support children's school progress as well as parental educational expectations (Catsambis, 2001; El Nokali, Bachman, \& Votruba-Drzal, 2010). Grolnick and Slowiaczek (1994) described parental involvement in a multidimensional way including a behavioural aspect (taking place at home or school, such as supporting homework or communication with teachers), a cognitive-intellectual aspect (e.g., bringing the child in contact with challenging learning activities) and a personal aspect (e.g., attitudes and expectations of parents about the importance and usefulness of education).

Within this framework, the beneficial influence of parental involvement on children's motivation and achievement is well established (Grolnick, Friendly, \& Bellas, 2009; Hill \& Tyson, 2009). For instance, Cheung and Pomerantz (2011) investigated parental involvement 
(e.g., discussing schoolwork, or communication with teachers), in students' learning in the United States and China in four waves with a six-month interval between the assessments. The results supported that children's perceived parental involvement was positively linked to children's engagement (e.g., time spent on schoolwork, use of SRL strategies as measured by a 30-item questionnaire assessing children's cognitive and metacognitive strategies) and achievement for both countries. In the same vein, Grolnick, Kurowski, Dunlap, and Hevey (2000) found that maternal cognitive (the frequency that mothers participate in activities with children at home) and personal involvement in the sixth grade (whether mothers are interested and informed about their children's school experiences) were protective factors of students' reading grades over the transition to junior high, and parental cognitive involvement was a protective factor for students' perceived competence. In addition, maternal personal involvement was positively linked with lower learning problems while maternal autonomy support (e.g., offering choices as opposed to being controlling) was positively linked to children's behavioural adjustments at school. However, the meta-analysis of Patall, Cooper, and Robinson (2008) reported mixed findings regarding the links between parental involvement and academic achievement. Moreover, a previous meta-analysis by Fan and Chen (2001) provided evidence that the relationship between parental involvement and children's school performance was stronger when performance included a general indicator of academic achievement and not the achievement scores in a particular course.

Parental involvement is not only related to students' academic achievement but also to their motivation. Puklek Levpušček and Zupančič (2009) examined the effect of students' perceptions about parents' and teachers' involvement in students' motivation and performance in math. Parental involvement included pressure, support (as "parents' interest in adolescent school learning and their encouragement of academic effort") and help. Students' perceptions about their teacher's behaviour involved support (e.g., praise, responsiveness) academic press [e.g., 'teachers' pressure for understanding, thorough thinking” (p.552)] and mastery goal (e.g., teachers' support on students to increase competence). The results showed that both teachers' and parents' involvement predicted students' motivation and math achievement. Concerning parents, their academic support was positively correlated with students' motivation. Parental pressure was negatively correlated with student's achievement, and it was the strongest (negative) predictor of students' mastery goal orientation, academic self-efficacy and performance in maths. It has also been suggested that parental support of children's autonomy and parental controlling behaviours (e.g., pressure, intrusiveness or dominance) during homework were differentially associated with 
students' mastery and performance goal orientations (Gonida \& Cortina, 2014). Specifically, parental autonomy support positively predicted students' mastery goal orientation while parental interference negatively predicted children's mastery goal orientation. Parental control positively predicted students' performance orientation.

Other aspects of students' motivational skills, such as persistence and effort, have also been investigated in relation to parental autonomy support. For example, in their review, Gonzalez-DeHass, Willems, and Holbein (2005) discussed the literature findings concerning the relationship between parental involvement, defined as "parenting behaviours directed towards children's education" (p. 101), and students' motivation. Parental involvement included parent-teacher interactions, involvement in school and home activities (e.g., homework), involvement in student's after-school activities, helping in choosing school courses, being informed about children's progress or grades, parental overcontrolling behaviours or autonomy support at home. The authors reported that parental involvement was positively associated with students' effort, concentration and attention. Students have a more inherent interest in learning and higher perceived skills. Moreover, parental over-control of the school work and the use of external incentives were associated with the development of students' extrinsic motivation, while parental encouragement and praise were associated with students' intrinsic motivation. The authors commented that when parents are involved in their children's education, students are more likely to become mastery oriented, and, therefore, to show persistence towards challenges and more satisfaction with their progress.

With reference to pre-school children's academic skills, the findings are consistent with those mentioned above. For instance, Arnold, Zeljo, Doctoroff, and Ortiz (2008) reported that parental involvement as assessed by the teacher during preschool education (including questions about parent-teacher cooperation and parent-child educational activities) is positively linked to children's preliteracy skills (receptive vocabulary, expressive vocabulary, auditory skills). Likewise, Fantuzzo, McWayne, Perry, and Childs (2004) found that home-based involvement activities (e.g., active encouragement of learning at home, reading to children, provision of learning opportunities) was the most significant predictor of preschool skills, such as teachers' rated motivation (e.g., interest about activities, a drive to understand and be successful in those activities), teachers' rated attention/persistence (e.g. maintain attention to a stimuli and keep on trying in difficult tasks) and receptive vocabulary rated by the examiners' independent assessment.

The above studies indicate that parental involvement in children's learning, especially in the form of providing autonomy support, is positively related with student's achievement 
and academic skills. However, it seems that there should be a distinction between parental involvement in school and parental involvement in learning. Harris and Goodall (2008) mentioned that parental involvement in school has a "social and community function" while parental engagement in children's learning at home is probably more associated with positive outcomes in children's learning. In this frame, studies have attempted to identify the way parents could support their children's learning examining different aspects of parental support, described in the following section.

\subsubsection{Parental cognitive and metacognitive support, children's learning skills and performance}

In this section, the relations between parental cognitive and metacognitive support and children's learning skills and academic performance will be examined. First, the links to children's cognitive and metacognitive skills will be presented followed by children's motivational and emotional skills.

Children's cognitive and metacognitive skills. Much work for the potential role of parental cognitive and metacognitive support in children's cognitive skills and achievement has been carried out. Data from several studies suggest that maternal supportive behaviours (e.g., quality of instruction, structure or manner of instruction) during problem-solving are positively linked to children's attention skills, intellectual ability, general executive function and methods of working or seeking assistance (Englund, Luckner, Whaley, \& Egeland, 2004; Hammond, Müller, Carpendale, Bibok, \& Liebermann-Finestone, 2012; Pianta, Nimetz, \& Bennett, 1997; Stright et al., 2001).

Within this framework, Stright, Herr, and Neitzel (2009) investigated, among other factors, the relationship between maternal cognitive support during problem-solving and children's reasoning abilities in the classroom in Hmong families. Parental support (cognitive support, directiveness of instruction, praise and criticism) was assessed while mothers provided help to their children in problem-solving tasks. Teachers were also asked to evaluate children's reasoning abilities, their conscientious, autonomous behaviour and persistence in work at the end of the kindergarten year by filling out a questionnaire. The results showed that maternal cognitive support was a positive predictor of children's reasoning skills in kindergarten even after controlling for maternal reasoning skills and education. 
The relations between parental scaffolding and children's reading, comprehension and language skills have also been examined. In this context, Clarke-Stewart and Beck (1999) investigated five year's old children's abilities in describing a video story (that they have previously watched and discussed with their mothers) and maternal scaffolding strategies during the joint task. The researchers found a relation between children's quality of stories and maternal scaffolding behaviours. Children in the mother-discussion group (compared to those in the no-discussion group) tended to provide more information about the story and had a greater comprehension of internal states when mothers oriented their joint attention to the story, used questions and clarifications, discussed the heroes emotions, corrected the child's misconceptions, and discussed in depth and detail the events of the story. Skibbe, Behnke, and Justice (2004) examined the verbal interaction between mothers and children (with language difficulties) during storybook reading tasks. The researchers sought to address the way and the stability of maternal assistance during a reading story task ( four times in 1-week period) and the relation between maternal scaffolding behaviours and children's phonological awareness skills in the above domain. Nine questions were provided to the mothers of the study to teach their children phonological awareness concepts. Maternal scaffolding of children's performance in the phonological awareness tasks was either directive (question restatement, the direction of child's attention, multisensory cue, prompting question), responsive (praise, phonological cue, extension, answer restatement), negative response or other. The study showed that mothers, in general, preferred a more responsive scaffolding (phonological cue and praise/affirmation). Moreover, mothers who provided more scaffolding that decreased over time including a decrease in directives had children who had greater performance in the tasks.

Recently, the longitudinal study of Devine, Bignardi, and Hughes (2016) explored children's executive function (e.g., conflict inhibition, working memory) as a mediator in the link between parental behaviour and children's academic ability, such as literacy and numeracy skills. Parental behaviours included parental scaffolding (parents' use of the contingency rule), the negative interplay between parent and child and parental provision of informal learning opportunities. Parents and children participated in two visits almost 1 year away from each other. The findings showed that children's executive function at Time 2, and not general cognitive ability, was a mediator in the relation between parental scaffolding at Time 1, negative parent-child interplay at Time 1 and children's academic skills at Time 2. Regarding parental opportunities for learning at Time 2, it was found that there was a direct association with children's academic skills. The authors concluded that parental scaffolding 
and negative interplay influence children's academic ability through their effect on children's emerging executive function.

The significant role of contingent responsiveness was underlined by Mol and Neuman (2014). Their study explored parent-child interaction during book reading, parental SES, and kindergarten children's receptive and expressive language skills. Parental strategies included labeling, generalizing, repetition and paraphrasing, scaffolding, lexical richness and contingent responsiveness. The findings supported that parental lexical richness, contingent responsiveness and in some cases labeling were positive predictors of children's language skills. Furthermore, contingent responsiveness was of particular importance as it mediated the effect of SES on children's language skills, highlighting the positive impact of both factors on children's language skills. The significance of autonomy promoting speech, contingent and cognitive stimulating language in young children's cognitive development was also highlighted by Mermelshtine and Barnes (2016). An interesting result was found in the study of Robinson, Burns and Davis (2009) who investigated the relations between maternal scaffolding and attention regulation in children living in poverty. Their findings provide evidence that mothers are informed about their children's performance skills and can diversify their scaffolding behaviours taking into account their children's needs at the specific time point. The study revealed significant differences in the type of parental scaffolding according to children's performance on the child-alone task. Children who did not achieve the maximum performance were given more verbal assistance, more questions regarding strategies, more verbal hints and prompts.

Parental cognitive and metacognitive support and the relations with children's metacognitive skills is another area of investigation. Parental support of learning and particularly metacognitive content and manner of instruction constitute a considerably valuable way to demonstrate and spread metacognitive information (Neitzel \& Stright, 2003). In general, the content and the type of parental instructions provide the foundation for children's metacognitive skills needed to regulate their learning, to make references to their way of thinking, to monitor their progress and to seek assistance in the classroom environment. The importance of promoting children's metacognitive thinking was supported by the study of Moss and Strayer's (1990) who investigated the provision of maternal metacognitive support to gifted and non-gifted preschoolers. The findings of their study showed that it was more likely for mothers of gifted children to promote the use of metacognitive strategies early in life as well as to encourage metacognitive thinking compared to mothers of non-gifted children. Other studies have also supported that children 
demonstrate better academic skills (e.g., spatial, arithmetic) and metacognitive skills (e.g., talk about their thinking) when parents give proper instructions, stimulation and assistance, metacognitive information (e.g., parents ideas or justification for the use of a strategy) and details in small manageable steps (Casey, Dearing, Dulaney, Heyman, \& Springer, 2014; Mattanah et al., 2005; Stright et al., 2001). It seems this kind of parental support offers children essential aspects of thinking even in primary school years with relative findings depicting that children whose parents provided appropriate instructions were more likely to control and monitor their learning and discuss their thinking in the classroom (e.g., Neitzel \& Stright, 2003).

Children's emotion regulation and motivation. Even though cognitive and metacognitive aspects of parental support of learning have been shown to be positively associated with children's cognitive-metacognitive skills and achievement, their relations to children's emotion regulation are not granted. For example, Erickson, Duvall, Fuller, Schrader, MacLean, and Lowe (2013) examined maternal "Simple scaffolding" (e.g., labelling the child's or her own actions) and "Complex scaffolding" (e.g., association or comparison of objects) and toddlers' emotion regulation. They found that maternal scaffolding was positively associated with children's emotion regulation as well as with mutual enjoyment during play but only for the preterm children; this relation was not significant in the full term toddlers. In the same way, negative affect was negatively associated with maternal scaffolding but only for the preterm children.

On the other hand, Pianta, Nimetz, and Bennett (1997) reported that maternal quality of instruction (e.g., initial orienting instruction, well-timed hints) was positively correlated with the working habits and overall competence of kindergarten children living in risk (e.g., low family SES or developmental skills). Fender, Richert, Robb, and Wartella (2010) observed parents with their infants discussing during co-watching an educational DVD. Parents were grouped according to their talking, as high teaching focus parents ("high amounts of target word-specific as well as general DVD talk and a low proportion of talk unrelated to the DVD", p. 619), moderate and low teaching focus parents. This study indicated that children's engagement with the task and children's use of target and new words were higher for those children whose parents were in the high teaching focus group. The researchers commented that in children less than two years old, parental intervention is essential for their learning during such activities. Finally, Hustedt and Raver (2002) found that low-income mothers who provided lower levels of verbal support, manual help and 
modelling had children who demonstrated lower levels of persistence and attendance in the task.

Even though, together this evidence underlines a critical role for parental cognitive and metacognitive support on children's learning skills and achievement, some methodological issues need to be discussed as they affect the generalisation of the above findings. For instance, different aspects of parental cognitive and metacognitive support were assessed to each one of these studies, such as the quality and manner of instruction, directiveness, cognitive support, lexical richness and contingent responsiveness. Likewise, children's cognitive and metacognitive skills included intellectual ability, executive function, reasoning, language skills, metacognitive thinking while children's motivational skills mainly involved children's working habits, engagement and competence. Moreover, in some cases, children's skills were assessed by teachers' questionnaires or observation during classroom activities. Furthermore, in a number of studies, it has been shown that the relations of parental behaviours and children's skills were not direct linear, but they were mediated by other factors, such as children's executive function. Concerning the sample, several studies addressed participants with specific characteristics, such as children with language difficulties and families or children living in poverty. These methodological differences challenge the generalization of the findings. This thesis adopted a micro-level approach, investigating different aspects of parental supportive behaviours and children's SRL skills. Concerning the parental cognitive and metacognitive support, this study assessed behaviours such as the mothers' attempts, hints and prompts to help the child understand the task and its characteristics, to strategically plan the activity, to employ strategies, to self-monitor and evaluate the cognitive outcome. These maternal behaviours, as well as the children's SRL skills, were assessed during actual problem-solving tasks. Thus, more information and a better understanding will be provided concerning the relations between specific aspects of maternal cognitive and metacognitive support and SRL skills in preschool children.

\subsubsection{Parental emotional-motivational support and children's learning skills and performance.}

Parental emotional support during joint problem-solving is also critical for children's learning. Ratner and Stettner (1991) wrote that "At a global level, emotional tone may influence whether a task is attended to at all" (p. 8) and they continued supporting that "Children may need to rely most on affective cues when functions are unclear, perhaps often 
in situations when parents do not instruct intentionally because the functions and significance of the adult's behaviour may be more implicit" (p. 9). In particular, taking into account the demanding nature of cognitive management, it seems that by providing emotional support, parents prepare and promote the motivational atmosphere to assure the activation of their children's SRL skills (Eccles, Wigfield, \& Schiefele, 1998, as cited in Neitzel \& Stright, 2003).

Children's cognitive skills. Merz, Landry, Montroy, and Williams (2017) focused their attention on the examination of bidirectional links between parental responsiveness and young children's executive function skills in low SES families. Parental responsiveness included measures of emotional and cognitive support and specifically warm acceptance, contingent responsiveness and verbal scaffolding. The findings indicated that parental responsiveness was a positive predictor of children's delay of inhibition and conflict executive function over time, but it was also depicted that only higher levels of delay of inhibition predicted increases in parental responsiveness over time. Consistent with the above results, the study of Razza and Raymond (2013) found positive relations between early maternal sensitivity and 54 months old children's delay of gratification. However, Bernier, Carlson, and Whipple (2010) reported no relations between early (12-15 months old children) maternal sensitivity and 18 months old children's categorization skills and marginal relations to children's working memory. Early maternal autonomy support was positively correlated with both the above factors at 18 months. No links were found between maternal sensitivity and 26 months old children's impulse control. In addition, even though maternal sensitivity and autonomy support were positively related to 26 months old Conflict EF (eg., working memory, inhibitory) the regression analysis (including as predictors the child's cognitive functioning, maternal sensitivity and autonomy support) showed that only autonomy support was an independent predictor of children's Conflict EF.

The relations between parental emotional response and children's language skills have also been examined. For example, a study that examined the effects of a brief motivational intervention (Family Check-Up) on the parenting behaviours of high-risk families and on young children's skills found, among other results, that parental positive behaviour support (e.g., parental involvement, positive reinforcement, engaged interaction, proactive parenting) when children were two years old was indirectly and positively linked to preschool children's language skills when they were four years old (e.g., following directives and answering questions, describing actions, sequencing events) through children's behavioural selfregulation when they were three years old (Lunkenheimer et al., 2008). In addition, the same 
study showed that parental positive behaviour support when children were three years old was positively associated with children's language skills at age four. Tamis-LeMonda, Kuchirko, and Song (2014) proposed some mechanisms that explain why parental responsiveness promotes young children's language skills. It was argued that parental responsiveness helps children understand that words and meanings can be shared and that are connected with the real world through the contiguity and contingency of the responsiveness. Moreover, responsiveness has also an educational aspect which promotes children's language skills by exposing them to the lexical variety. Finally, parents support their children by adjusting their language to the developmental needs of their children.

Other aspects of emotional-motivational support, that is parental warmth and nurturance (e.g., being loving and responsive), have been found to be positively associated with some aspects of children's help-seeking (Puustinen, Lyyra, Metsäpelto, \& Pulkkinen, 2008). For girls, parental nurturance ("the extent to which parents report being loving, responsive, and involved") and parental warmth were positively associated with longer thinking times before asking for help, negatively associated with questions that aimed to avoid the task and positively to a minor capacity to reuse the help they have previously accepted on similar tasks (hence, they asked for help again). On the other hand, the study showed that paternal emotional warmth was positively related to boys' inefficient helpseeking behaviours (e.g., unnecessary questions, questions not aimed at understanding).

Children's emotion regulation and motivation. Parental emotional support also refers to the encouragement and motivation that children might need to complete difficult tasks. It is possible that parental praise and criticism may affect children's motivation to continue solving the task or to show persistence when dealing with challenging situations. Some studies have focused on the relationship between parental emotions (tenderness, warmth), children's motivation and children's self-perceptions of their competence. For instance, positive relations between parental warmth and children's self-efficacy have been reported (Richman \& Rescorla, 1995). In addition, the study of Salonen, Lepola, and Vauras (2007) reported that parents, whose children were task-oriented, were better at adjusting their emotional reactions in accordance to their children's emotions and provided more positive emotional support.

Similarly, Hokoda and Fincham (1995) examined mother-child interaction patterns in a group of elementary children, grouped as mastery oriented or helpless. The results revealed that mothers of mastery-oriented children significantly differed from mothers of helpless children in the degree of sensitivity and responsiveness towards their children's inquiry for 
help. Mothers of mastery-oriented children showed more positive affect, provided more task focused strategies and redirected the helplessness feelings of children during the difficult tasks. On the contrary, mothers of helpless children were less likely to respond to their children's request for help and were more likely to encourage the feelings of helplessness to their children, suggesting them to quit or move on to the next task while expressing negative feelings. One more study conducted by Devlin (2012) provided more data on the subject investigating the relation between maternal affect (e.g., verbal tone) and sensitivity (e.g., quality of assistance, being supportive) and toddlers' persistence. The results showed that maternal affect was not associated with children's persistence. However, maternal quality of assistance and maternal support of development (defined as "Mother's ability to be encouraging and sensitive to when her child is in need of help while encouraging mastery by refraining from intrusive behaviour"), were significantly associated with children's persistence. On the contrary, Stright et al. (2009) reported that maternal praise and criticism did not predict Hmong children's persistence as assessed by the kindergarten teacher.

Intervention studies. Aspects of maternal emotional support have also been examined in the context of intervention programs. Within this framework, Landry, Smith, and Swank (2006) examined, among other hypotheses, whether increases in mothers' responsiveness (e.g., contingent responsiveness, emotional support, and quality of language) will positively affect the cognitive, communication, affective and social skills of infants born at term or at very low birth weight. In general, the results showed that the increases in maternal responsiveness (noticed in mothers in the intervention group) were positively linked to children's social, emotional, communication and cognitive skills. The researchers highlighted the importance of parenting interventions aiming at supporting and promoting this parenting aspect.

In the same line, Obradović, Yousafzai, Finch, and Rasheed (2016) investigated factors that could account for the positive effects of an early Responsive Stimulation Intervention on four years old children's cognitive skills. Children came from highly disadvantaged families in rural Pakistan (sample consisted of 1302 children at the beginning of the study). In fact, this study supported that early intervention in parents had positive longitudinal effects on preschoolers' cognitive skills. Among other findings the study showed that the current home stimulation quality and the previous and concurrent maternal scaffolding (e.g., sensitivity, contingent responding, helping the child maintain interest) were independent mediators of the intervention effects on four years old children's skills, controlling for SES risk and personal factors (e.g., gender). Moreover, the impact of the 
intervention on children's executive function and performance intelligence was direct and more central compared to other covariates. As in the previous study, the researchers of this study also stressed the importance of implementing programs that target parental scaffolding behaviours to assist the development of children's skills.

The evidence presented in this section indicates that parental emotional and motivational behaviours are aspects of parental support with different conceptualisation across various studies (e.g., warmth, sensitivity, positive reinforcement and involvement, affection and nurturance). As presented in this section, some of the previous research in the field has shown positive relations between parental emotional-motivational support and students' learning, motivational and academic skills. However, other studies either report mediation relationships between them or they did not find such links between maternal sensitivity and aspects of children's executive function, or between maternal affect, praise and criticism and children's persistence. In addition, regarding children's skills, the literature is so broad regarding their assessment. For instance, different aspects of students' emotional and motivational skills were investigated in the studies above; some of them included children's persistence, students' self-efficacy, task or mastery orientation. As discussed in the previous section, these differences in the concepts assessed and the adopted methodologies do not advance the generalization of the findings. Moreover, studies that use an observational and a micro-analytic approach to examine specific parental aspects of parental support and children's SRL skills are scarce. Therefore, the present study will examine maternal emotional and motivational support referring to mother's verbal and non-verbal behaviours, such as the recognition of child's efforts, the mother's attempts to attract and maintain the child's interest to the task and the lack of criticism, disapproval, or rejection of the child's efforts. Adopting a micro-analytic and longitudinal approach, the study will examine the relations between the above maternal behaviours and children's SRL skills.

\subsubsection{Parental boosting the child's autonomy, parental control and children's learning skills and performance}

Parental gradual transferring the task's responsibility to the children constitutes another important aspect of parental support (Stright et al., 2009). It is claimed that parents should aim at encouraging children's autonomy and independent problem-solving by providing opportunities for independent action and by giving more responsibilities and fewer instructions to the child (Neitzel \& Stright, 2003; Wood \& Middleton, 1975). The available 
data show that the use of prompts, questions and hints on behalf of mothers, promote children's dynamic function in the learning process, compared to readily giving the answer to the children, over-controlling the children's steps or taking on the solution of the task (Neitzel \& Stright, 2003). A number of studies have postulated that maternal autonomy support is positively linked to various children's outcomes, such as homework-like task performance, social and academic adaptation, executive function and achievement in reading (e.g., Bernier, Carlson, \& Whipple, 2010; Grolnick, Gurland, DeCourcey, \& Jacob, 2002; Joussemet, Koestner, Lekes, \& Landry, 2005). Likewise, Cooper, Lindsay, and Nye (2000) reported that students' grades in class and in standardized tests were positively associated with parental autonomy support (e.g., allowing the child to do the homework that should be done by the child) and negatively with direct involvement (e.g., the frequency that homework required someone to participate or help).

On the contrary, parental behaviours, such as parents' pressure for obtaining or maintaining grades, limited freedom in decision making, excessive participation in children's activities and low involvement seem to be linked to negative outcomes on some aspects of children's adjustment (e.g., intrinsic motivation) and performance (e.g., Fan \& Williams, 2010; Kramer, 2012). It has been demonstrated that higher maternal help of homework was negatively related to children's academic performance progress in Grade 4, particularly when mothers perceived their children as not capable for autonomous work (Silinskas, Kiuru, Aunola, Lerkkanen, \& Nurmi, 2015). Studies exploring the above link suggest that mothers' controlling instructions (e.g., behaviours that interfere with the child's current activity or the pressure on the child to act as the parent wants) (Deci, Driver, Hotchkiss, Robbins, \& Wilson, 1993; Gurland \& Grolnick, 2005), parents' close supervision of school work (in the form of high surveillance of homework), unconstructive responses to failure (Ginsburg \& Bronstein, 1993) and the use of task-extrinsic consequences (Gottfried, Fleming, \& Gottfried, 1994) are negatively linked to patterns of children's motivation (e.g., intrinsic), interest and low academic performance. Likewise, the study of Karbach, Gottschling, Spengler, Hegewald, and Spinath (2013) explored students' perceived parental involvement (support of autonomy, emotional responsivity, structure and control that focuses on achievement) and students' general cognitive ability as predictors of performance in language and mathematics. The results showed that general cognitive ability was the best predictor of students' academic performance. Moreover, while parental support of autonomy and emotional responsivity did not predict students' performance, parental control focused on achievement and structure (e.g., parental expectations in relation to learning) negatively affected academic performance. 
Some studies suggest an association between children's motivation and parental autonomy support. For instance, Knollmann and Wild (2007) reported interesting relations between students' perceived parental control levels, students' motivation and emotions towards school work. The findings indicated that students adopting an extrinsic motivation perspective were more likely to report negative emotions during homework when they were encouraged by their parents to act autonomously, compared to students with an intrinsic motivation perspective. The researchers suggested that children with extrisic motivation may felt better in firmly organised environments that guarantee that their extrinsic motivation will be fulfilled. On the other hand, children adopting an intrinsic motivation perspective reported positive emotions in the autonomy condition compared to the directive-instruction condition. This study supported that parental quality of support is differentially associated with students' emotions during learning according to children's motivation orientation.

In addition, Gonida and Cortina (2014) found that parent autonomy support (e.g., facilitating hints, promoting self-regulatory practices) mediated the relation between parent mastery goal and students' mastery orientation while parental control slightly mediated the relation between parent performance goal and students' performance orientation. Similarly, Koutsoulis and Campbell (2001) showed among other findings that parental pressure (e.g., parents demanding behaviour on children to maintain high grades) negatively predicted students' motivation (self-concepts, attitude toward school, and educational aspirations) and achievement in math and science. On the other hand, parental psychological support (e.g., creating a psychologically supportive atmosphere at home, parents' feeling of being proud), had a positive and direct impact on students' math and science self-concept, attitude toward school, and educational aspirations and an indirect on students' achievement.

In addition, Mih (2013) explored a model of relations between students' perceptions about their parents' supportive behaviours and children's behavioural engagement in school, strategy use and achievement. This research focused on two distinct aspects of perceived support, a) parental autonomy support and b) parental psychological control defined as "parental controlling behavior that intrudes into the psychological development of the child" (p. 44). The results indicated that parental autonomy support had a positive effect on children's performance through the mediation of children's autonomous motivation, persistence and effort. The impact of parental psychological control on children's performance was entirely mediated by children's procrastination, controlled motivation and disorganisation. 
The above studies indicate that there is a positive association between children's performance, motivational skills (e.g., persistence, effort), executive function and parental autonomy support and a negative association with parental overcontrolling behaviours. In general, the above definitions of parental autonomy support and parental overcontrolling are close to the present study's conceptualisation of parental autonomy support and overcontrolling behaviours. In the context of this thesis, maternal autonomy support refers to the mother's encouragement of the child's independent cognitive involvement in the solution process and the mother's avoidance of interfering and controlling the behaviours of the child. This maternal aspect of supportive behaviour (autonomy support) was found to be consistently and positively related to children's SRL skills and performance. There are rather consistent findings in the literature that underline the significance of this particular aspect of parental support in various learning contexts (e.g., problem-solving, homework involvement, school settings).

\subsubsection{Studies exploring both cognitive-metacognitive and emotional-motivational parental support}

Even though some of the studies described above examined both cognitive and emotional aspects of parental support of learning, there are few multidimensional studies that are worth mentioning in a distinct section, as they are methodologically closer to the present study.

Bearing this in mind, Leerkes et al. (2011) commented that most research in the context of parental scaffolding shows that parental behaviours during problem-solving are associated with children's academic skills. Nonetheless, it is not yet understood the unique importance of the cognitive versus the emotional parental supportive behaviours during problem-solving. In addition, it is not clear if the positive relations between parental cognitive and emotional support during problem-solving and children's learning skills reflect general positive parental characteristics (e.g., parents could be more cognitively challenging and emotionally responsive in general). These researchers also mentioned that it is possible, that parents who offer more cognitive support to their children during problem-solving to have the tendency to provide more cognitive stimulation in general, and, likewise, parents who support their children emotionally, to be in general more sensitive and responsive to the emotional needs of their children. Therefore, these general traits of parenting might be responsible for promoting the development of children's skills. Taking that into account, they 
highlighted the need to examine parental cognitive and emotional support during problemsolving simultaneously considering other factors too, such as the quality of the learning environment at home and the overall parental emotional responsiveness to better understand the specific contribution of different factors. In this respect, Leerkes et al. (2011) studied 263 child - mothers pairs examining whether parents' emotional and cognitive support during joint problem-solving with their three years old children, the quality of the learning environment and maternal emotional response, were predictors of children's academic skills one year later. The findings indicated that maternal emotional and cognitive support during joint problem-solving, the quality of the learning environment at home and the overall emotional response of the mother when the child was three years old were positively associated with children's pre-academic skills (numeracy and language skills, work habits) one year later without controlling for other variables. When children's pre-academic skills at three years of age and demographic variables were controlled, only maternal emotional support and home learning environment were positively correlated with children's preacademic skills. Maternal emotional support and quality of the learning environment at home positively predicted children's pre-academic skills one year later. In addition, when interaction effects were examined, only the interaction effect between maternal emotional support and children's pre-academic skills at three years of age was significant. The positive effect of emotional support on children's pre-academic skills at age four was apparent in children whose abilities were weaker at the age of three.

Such a study that adopted a multidimensional view of parental behaviours in a problem-solving context is a recent work by Zhang and Whitebread (2017). These researchers explored parental scaffolding behaviours and children's self-regulated learning (cognitive, metacognitive, motivational) in 130 Chinese kindergarten children. Parental scaffolding behaviours included parental cognitive support, emotional support and contingency. Cognitive and emotional support was assessed using an adapted version of Neitzel and Stright's (2003) coding tool. Children's self-regulated learning skills were assessed using a coding scheme adapted from the Strategic Behaviour Observation Scale by Dermitzaki et al. (2009) and took place three weeks after the assessment of parental scaffolding behaviours during joint problem-solving. The findings of the study revealed that parental cognitive support was positively and significantly associated with children's cognitive and metacognitive skills, but not with their motivational skills and cognitive performance. Maternal emotional support was positively and significantly correlated with all of the children's SRL skills but not with their cognitive performance. Parental contingency 
was positively and significantly associated with all of the children's SRL skills and performance. However, only parental contingency was an independent predictor of children's SRL skills and performance. At this point, it should be noticed that Zhang and Whitebread's (2017) study is of particular importance for the present thesis, as it examined similar concepts with the use of comparable methodological tools with those employed in this thesis.

Neitzel and Stright (2003) also investigated the impact of maternal cognitive, emotional and autonomy support during problem-solving on children's SRL skills at school. Their study found that the manner of instruction was not related to children's persistence when maternal emotional support was high. Manner of instruction was related to children's help-seeking behaviours in the classroom when maternal emotional support was high. In addition, maternal transfer of responsibility predicted children's persistence. Metacognitive information predicted children's monitoring progress, metacognitive talk and persistence when the information was delivered in an understandable way.

This section could not leave behind two significant studies which examined the relation between parenting behaviours and children's SRL skills. Pino-Pasternak and Whitebread (2010) provided a thorough review of studies that examined socioemotional and instructional parental behaviours and their links to children's metacognitive and motivational skills. Another aim of their work was to build a theoretical model which describes specific parental dimensions and behaviours which seem to be connected in different ways with metacognitive and motivational aspects of SRL. Their findings directed to the development of a model which underlined three parenting dimensions, that is Challenge, Autonomy and Contingency. The authors used a Venn diagram to demonstrate the three parenting areas and the way they were imbricated. Six parenting behaviours were found to be particularly linked to children's SRL skills, that is metacognitive talk, active participation, adult-child shifts in responsibility, understanding of control, emotional responsiveness and contingent instructional scaffolds. In this review, these six parenting behaviours were found to be related to various aspects of children's SRL skills. For example, parents' encouragement of metacognitive talk and encouragement of active participation were linked to children's metacognitive skills; adult-child shifts in responsibility, parental contingent instructional scaffolds, parental responsiveness and parental promoting internal understanding of control were linked to motivational aspects of SRL, such as self-competence and intrinsic motivation (see, for a review, Pino-Pasternak \& Whitebread, 2010).

This model was verified by Pino-Pasternak, Whitebread, and Tolmie (2010). This study is worth mentioning because it adopted a multidimensional approach to parental 
scaffolding. The researchers investigated the relationship between self-regulated learning of underachieving primary Chilean children and parental behaviours in authentic academic tasks during parent-child interaction and during child-alone activities two weeks after the interaction. These parent-child activities were employed in terms of a program of activities that promoted families to "engage in and talk about self-regulatory behaviours" (p. 225). Children's SRL skills were assessed before, during and after the program. They consisted of Metacognitive knowledge, Regulation of cognition (e.g., planning, monitoring), Mastery orientation (e.g., children's interest in learning and understanding), Helpless Orientation (e.g., frustration in the face of difficulties) (p. 226). Socioemotional (e.g., affect, responsiveness) and instructional (cognitive demand used in parental support, children's ongoing level of understanding and parental contingency) behaviours of parents and children were also examined as well as their association with children's SRL during the program's sessions and at posttest (the final assessment of children's SRL).

The findings of the study examining the parents' and children's socioemotional behaviours showed that children's metacognitive knowledge and regulation of cognition during mother-child activities were positively related to parents' positive affect. Mastery orientation was not associated with the parental behaviours. Children's helpless responses were positively related to parental negative affect. At posttest, similar relations were noticed. For instance, metacognitive knowledge at posttest was positively associated with the positive aspects of parental control. However, more clearly observed relations occurred during the interaction and not at posttest, with the authors commented that "these two sets of variables" (p. 251), that is the socioemotional behaviours and children's SRL are more closely related in the context of joint activities.

Regarding the instructional behaviours during the sessions, the study revealed that mastery orientation was correlated with average levels of parental cognitive demand and children's understanding. Metacognitive knowledge and regulation of cognition were correlated with higher levels of cognitive demand and children's understanding. At posttest, the relations remained significant. Metacognitive knowledge, regulation of cognition, and mastery orientation were associated with higher levels of cognitive demand and contingent support and medium to high levels of children's understanding whereas children's helplessness was related to low levels of cognitive demand.

The researchers commented that even though parental behaviours in general (socioemotional and instructional) seem to be associated with children's SRL skills, the posttest showed that the most evident links came out for contingent instructions, medium and 
high cognitive demand and medium and high levels of understanding. As Pino-Pasternak et al. (2010) pointed out "These results, therefore, suggest that while the socioemotional behaviours might have been important for setting the immediate tone of the interaction under which more or less adaptive instructional patterns were likely to emerge, it was mostly behaviours within the instructional dimension that might have carried the weight of SRL change" (p. 262).

The above studies illustrate how complicated the literature is, concerning the relationships between parenting and children's learning. The complexity of these relations is partially attributed to the different definitions and assessment methods of parental supportive behaviours adopted by the researchers. In broad, it is evident that the cognitive and emotional support provided by parents during problem-solving have a positive impact on children's skills, although some studies consider the cognitive support as more important while others highlight the emotional support. One more interesting area of research is the examination of which factors affect these parental behaviours. The following section will attempt to shed light on some variables that have been found to determine the way parents respond during parent-child interactions.

\subsection{Factors Related to Parental Supportive Behaviours}

Belsky (1984) in his process model regarding the determinants of parenting behaviours supported that there are three interacting factors that influence parents' behaviours, that is, Parental personal qualities (e.g., personality), the Social context (e.g., marital relations, work, social network) and the Characteristics of the child (e.g., temperament). In the following sections, factors that have been found to be associated with parental behaviours will be examined. These factors include children's temperament, age and gender, parenting style, parental self-efficacy, maternal education and the nature of the problem-solving tasks.

\subsubsection{Children's temperament and personality}

According to Goldsmith, in a discussion in the roundtable with other four theorists, temperament has some inclusion criteria which identify that "temperament is emotional in nature, that it pertains to individual differences, that it refers to behavioural tendencies rather than actual occurrences of emotional behaviour (and thus is relatively stable), and that it is indexed by the expressive aspects of emotion" (Goldsmith et al., 1987). Most recently, 
temperament was defined as the "constitutionally based individual differences in reactivity and self-regulation, as seen in the emotional, motor and attentional domains" (Rothbart \& Bates, 1998; Rothbart \& Derryberry, 1981, as both cited in Rothbart, Ellis, \& Posner, 2004, p. 357). Research in young children (3-7 years old) revealed three temperamental systems which tap Surgency or Extraversion, Negative Affectivity and Effortful Control (Rothbart, Ahadi, Hershey, \& Fisher, 2001). Rothbart, Posner, and Hershey (2006) supported that personality could initiate from these early discrepancies in temperament and that it is essential to understand the way temperament develops and evolves during the time so that we better understand psychopathology.

Even though previous studies have examined the relation between children's temperament and parenting, only a few have looked for specific parental behaviours during problem-solving, and the way parents adjust their support in reference to their children's temperament. One of them, the study of Fagot and Gauvain (1997), found that maternal ratings of children's temperament at 18 months predicted parenting behaviours 12 months later. In particular, mothers who perceived their children's behaviour as difficult tended to offer more cognitive support and behavioural directives, probably to keep them engaged in the task. This study replicated previous results by Gauvain and Fagot (1995). Their first study depicted that mothers who considered their children as having difficult temperament traits 7 to 15 months before the joint problem-solving tended to provide more cognitive assistance and disapproval to their 2,5 years old children. However, no relations were found between children's temperament and positive support. Their second study showed that children's difficult temperament at 18 months was positively associated with maternal cognitive support and behavioural directives and negatively with positive support when children were 30-35 months old. No relations were found between children's temperament and maternal disapproval. Neitzel and Stright (2004) reported that mothers who had higher levels of education provided more assistance (e.g., regulation of task difficulty, encouragement of children's active participation) to the children who were perceived as difficult.

The work of Stright, Gallagher, and Kelley (2008) indicated that children's temperament moderated the relations between maternal practices at early childhood (e.g., emotional and autonomy support) and children's academic competence, social skills and personal relationships in the first grade. Using longitudinal data, the study showed that the links noticed between parenting practices (e.g., emotional and autonomy support) and children's academic and social outcomes as rated by teachers were stronger in children characterised as difficult by their mothers. Their results provided support to the differential 
susceptibility hypothesis, which states that "children may differ in the degree which parenting experiences may affect development” (Belsky, 1997, 2005, as cited in Stright et al., 2008), supporting that children with difficult temperaments could be more vulnerable when confronted with negative parenting.

Children's compliance has also been investigated in relation to parental instructions. The study of Gauvain and Perez (2008) investigated different types of instruction provided by mothers during planning activities with their four to five years old children. Children formed two groups according to their level of compliance, either normal levels or high levels of noncompliance. The study showed that the way mothers provided instructions was changing according to the level of the child's compliance. In particular, mothers provided a more low level, directive and negative type of instruction in the noncompliance group while it was also noticed that mothers and children of this group shared less task responsibility.

In spite of the research interest in this area, the nature of the above relations has not yet been established. Some studies found evidence for bidirectional relations, proposing that it is possible for children's temperament to have an impact on parenting practices which in turn can influence children's temperament (e.g., Eisenberg, Fabes, Shepard, Guthrie, Murphy, \& Reiser, 1999; Lengua, 2006). On this subject, the literature shows that positive parental practices (e.g., warmth, coordination between parents) have been associated with better effortful control and self-regulation in children (e.g., Colman, Hardy, Albert, Raffaelli, \& Crockett, 2006; Karreman, Van Tuijl, Van Aken, \& Deković, 2008).

In general, research on children's effortful control has well demonstrated positive links to some parenting behaviours (e.g., expressivity, sensitivity) (e.g., Spinrad et al., 2007; Valiente et al., 2006). In another study, Eisenberg et al. (2010) examined the bidirectional relations between children's effortful control and parental teaching strategies (cognitive assistance strategies, directive and questioning) longitudinally. The findings showed that children's effortful control constantly predicted maternal teaching style across time with no any inverse relations. More specifically, children's effortful control at 18 months positively predicted maternal cognitive assistance, questioning approaches and lower maternal directives at 30 months, while children's effortful control at 30 months positively predicted maternal cognitive assistance and fewer maternal directives at 42 months.

Lengua, Honorado, and Bush (2007) examined in a short-term longitudinal study the impact of cumulative contextual risk on effortful control in preschool children and the role of parenting behaviours during interaction tasks in the above relation. The findings revealed that parenting characterised by clear and steady boundaries in noncompliant environments, 
effective responsiveness in children's negative affect as well as the respect for child's autonomy positively predicted children's effortful control. Cipriano and Stifter (2010) also explored whether and how parental behaviours and emotional tone affected toddler's temperament (inhibited or exuberant) and effortful control in preschoolers. The findings supported that mothers of exuberant children who showed more command/prohibited parental behaviours combined with a positive emotional tone, when the child was two years old, considered their 4,5 years old children to have more effortful control compared to mothers who demonstrated more redirection/reason parental behaviours and neutral tone. One more study showed that maternal observed warmth and sensitivity when children were 30 months predicted one year's later children's effortful control (Spinrad et al., 2012). One more study, conducted by Hustedt and Raver (2002) explored the relation between maternal scaffolding behaviours, joint attention and dyadic reciprocity in low-income, ethnic minority families. Maternal strategies included verbal strategies, modelling, manual help, solution, recruitment (e.g., children's attention), direction maintenance (words that help children to maintain their interest), verbal hints, positive and negative feedback, off-topic speech and no verbal instructions. The results revealed that the mothers of the children who were highly attentive were more likely to focus on the solution of the problem and be more involved and less likely to use off-topic speech. The fact that these group of highly attentive children were found to request more verbal assistance might propose that these children asked for their mothers' attention and assistance. Therefore, as the authors suggest, "scaffolding must be viewed from a dyadic rather than individually driven perspective" (p. 118).

Except for children's effortful control, children's negative affectivity, which includes irritability, fear, anger, shyness, frustration and discomfort (Gartstein \& Rothbart, 2003) has also been explored in relation to parenting. In this respect, studies report that negative emotionality (NE) or difficult temperament is linked to higher parental control. For example, the study of Braungart-Rieker, Garwood, and Stifter (1997) investigated the link between toddlers' temperament, type of maternal control and children's compliance behaviours. The findings showed that negative reactivity in children was negatively associated with maternal guidance and positively with maternal control. In addition, one more study examined the relations between children's temperament and parenting styles in Beijing, Chinese parents and western United States parents (Porter et al., 2005). For Chinese and US parents, a positive relation was found between children's emotionality and authoritarian style. However, cultural differences were also found concerning children's activity level, which was more 
associated with the authoritative style and less with the authoritarian style but only for Chinese parents.

Negative relations were also reported between children's difficult temperament and parental sensitivity (e.g., Feldman, Greenbaum, Mayes, \& Erlich, 1997; Mills-Koonce et al., 2007). Scaramella, Sohr-Preston, Mirabile, Robison, and Callahan (2008) explored the longitudinal relations between parenting quality and young children's reactivity. They found that although harsh maternal behaviours when children demonstrated non-compliance were a predictor of children's distress, the latter was not a predictor of harsh parenting. On the other hand, in the same study, supportive and contingent maternal behaviours were not a predictor of children's distress, but the latter negatively predicted maternal supportive behaviours.

Ghera, Hane, Malesa, and Fox (2006) reported positive relations between children's negative reactivity and maternal sensitivity but only when mothers considered their children's as having high soothability and negative relations when they considered their children's as having low levels of soothability. The study of Lengua and Kovacs (2005) explored the relations between children's temperament and parenting behaviours and their influence on children's internalising and externalising problems longitudinally. Children's were about 9.9 years old at Time 1. The results showed that after controlling for former children's temperament and parenting, children's fearfulness and children's positive affectivity were predictors of maternal acceptance and that maternal lack of consistent discipline was a predictor of children's fearfulness and irritability, suggesting the presence of bidirectional associations between children's temperament and parental behaviours.

Overall, it seems that the literature above suggests that there are significant links between children's difficult temperament and more directive parenting behaviours, poor effortful control and parental controlling behaviours, low responsiveness and sensitivity. However, it is also evident that the relations described above are multifaceted; these studies pointed out bidirectional and unidirectional links as well as mediation and moderation paths. Taken this information into account and the fact that these studies have used different tools to assess children's temperament and different aspects of parental behaviours, a general conclusion about how specific aspects of temperament relate to specific parental behaviours during learning could not be safely drawn. Further investigation is needed regarding the directions of these relations and the links between specific parental behaviours during problem-solving and children's temperament. 


\subsubsection{Children's age and gender}

Although the literature has provided some evidence concerning the relations between parental behaviours and children's age or gender, far too little attention has been paid to the links between child's age or gender and specific parental supportive practices during learning.

Studies suggest that some parental practices change according to children's age or gender while other practices remain stable. As an illustration, McNally, Eisenberg, and Harris (1991), in a longitudinal study, explored the stability of maternal practices regarding elevation (change in the mean level of the measures) and consistency (the emphasis that is given over time compared to other parents. The Child Rearing Practices Report was administered to mothers, examining parenting practices, five times, when children were between 9-10 years old, 11-12, 13-14, and 15-16 years old. In general, the results indicated a high level of consistency of maternal practices across time. Be that as it may, some parenting practices did change. Maternal control increased when children were in mid-adolescence while positive affect decreased over time. Regarding children's gender, mothers expressed more negative affect on boys over time. In addition, maternal emphasis on achievement increased as children grew older. One more study of Aunola and Nurmi (2004) examined the role of maternal affection in the relation between maternal psychological control and children's math performance from preschool to $2^{\text {nd }}$ grade (children's age at the beginning of the research was 5-6 years old). Mothers completed a questionnaire three times in three years, assessing their affection (defined as the "positive relation with the child"), behavioral control (defined as the "clear expectations regarding the child's behavior and the value of obedience in children") and psychological control (defined as "parental attitudes appealing to pride and guild and expressing disappointment"). Among other results, the researchers found that even though there were significant differences in the maternal measures individually, there were no significant differences in their "developmental trends". Foreland and Jones (2002) examined the longitudinal differences in maternal monitoring ("parents' perceptions of their knowledge about various aspects of their children's lives") and warmth in low-income innercity African-American families starting when children were almost eight years old. The assessments were implemented 13 months after the first assessment, 19 months after the second and 15 months after the third. The researchers reported an absolute decreased (comparison of mean levels) but not a relative one (correlations across assessments) in 
parental warmth and monitoring. With respect to children's gender, the study indicated that the monitoring of the girls was more consistent than the monitoring of the boys.

Nonetheless, data from several studies have supported the view that parental behaviours such as instructive and directive prompts, supervision and punishment vary in their level according to the age of children. For instance, Fagot and Kavanagh (1993) examined parenting practices in children 12-month-old and in children 18-month-old and found significant differences. Parents were more instructional and directive (e.g., instructed, demonstrated, asked or answered questions) with the older children than with the younger while positive interaction decreased (e.g., favourable comments, associative play). Loeber et al. (2000) reported changes in family practices in a longitudinal study for the antisocial and disruptive behaviours in children and adolescents that included boys. In particular, physical punishment decreased over time (absolute change) while poor supervision (e.g., knowing the friend of the child) and low positive parenting (e.g., smiles, approval) increased in time. Likewise, the study of Fagot and Hagan (1991) also reported that parents demonstrated more positive behaviours (positive physical behaviour, encouragement) with younger children (12 months old) and more directions and instructions as children grew older. More insights into the subject were given from the study of Plumert and Nichols-Whitehead (1996) who examined parental scaffolding behaviours in three and four years old children in regards to their spatial communication skills. They found that parents provided more directive prompts in three years old children than in four years old children. In their second study, they found that the directive prompts were helpful for both children's age groups but for the youngest children nondirective prompts were less effective.

Considering children's gender, some evidence has been already reported in the above studies (e.g., Forehand \& Jones, 2002; McNally et al., 1991) indicating that some but not all of the studied parental behaviours differentiate according to the gender of the child. Likewise, in one more study that explored parental involvement and children's achievement showed that parents were considered to exercise more academic pressure (e.g., consequences if children have bad grades) on boys than girls. Girls, on the other hand, experienced more parental management of their learning environments (e.g., learning activities at home). However, no differences were found regarding participation with homework and encouragement and support for learning (Rogers, Theule, Ryan, Adams, \& Keating, 2009).

Some variations in parental talk according to children's gender and task have also been reported in the literature. For instance, Tenenbaum and Leaper (2003) investigated the conversations between parents and their 11 and 13 years old children about science. Parent- 
child dyads took part in science and non-science tasks. The results showed that during the physic task, the fathers of the study (but not the mothers) used more cognitively demanding talk (e.g., conceptual question, explanation, scientific vocabulary) with the boys than with girls and the same level of cognitively demanding talk during the interpersonal task (e.g., providing solutions to a social problem). However, in the computer task (technology) both parents offered the same level of cognitively demanding speech to both boys and girls.

Given all that has been discussed above, the findings suggest that some of the parenting practices are inconsistent across different periods of children's life. Still, the studies in this section examined general parental behaviours in different children's ages, and they also used different methodologies regarding the assessment of these parental behaviours. Similarly, regarding children's gender, the results are not consistent, with some studies showing that parents differentiate their behaviours according to children's gender while other studies do not mention such kind of differences. Whether and which one of the parental behaviours change in joint learning experiences during the preschool period or according to the child's gender is an interesting area of research. The current study will attempt to detect such changes in maternal behaviours by examining mother-child problem-solving interactions at two-time points. Such information is significant for both teachers and parents in order to be aware and awake for potential changes in their behaviours and the potential effects on children's learning.

\subsubsection{Parenting styles}

The term "parenting style" was defined as "a constellation of attitudes toward the child that are communicated to the child and that, taken together, create an emotional climate in which the parent's behaviours are expressed" (Darling \& Steinberg, 1993, p. 488). According to Baumrind (1978), authoritarian parents are considered to be rigid, instructive and emotionally disconnected. They acquire conformity, and they are irritated with their children's inappropriate behaviours. "The authoritarian parent believes in keeping the child in a subordinate role and in restricting his autonomy, and does not encourage verbal give and take, believing that the child should accept a parent's word for what is right" (p. 244). Permissive parents do not provide sufficient constraints or control to their children. On the contrary, they are characterised as overly loose on their expectations and tolerance levels. "The permissive parent sees him- or herself as a resource for the child to use as he wishes, but not as an active agent responsible for shaping and altering the child's ongoing and future 
behaviour" (p. 244). Finally, authoritative parents provide coherent and rigid instructions, and they are highly demanding, but they combine their discipline methods with compassion, affection, communication support, logical arguments and boost for autonomy (Baumrind, 1978).

In general, it is suggested that when studying parenting styles, the cultural context should be taken into consideration. For example, Rudy and Grusec (2006) explored the link between parental authoritarianism and parents' cognitions and emotions in two groups, Western European (individualist group) and Egyptian, Iranian, Indian and Pakistani (collectivist group). The findings supported the view that authoritarianism has a different meaning in these two groups, as it was correlated with negative parental emotion and cognition ("less adaptive maternal thoughts") only in the individualistic group.

In general, the very few studies that have explored the relations between parenting styles and parental support during learning showing that authoritative parents tend to be more contingent to their children needs and provide more efficient scaffolding during problemsolving. In particular, a study conducted by Pratt, Kerig, Cowan and Cowan (1988) searched for relations between parental style and sensitive tutoring during problem-solving and found that authoritative parents provided more appropriate scaffolding that was contingent with their child's needs than non-authoritative parents. Consistent with the above findings, Pratt, Green, MacVicar, and Bountrogianni (1992) tried to explain the link between parenting styles and children's school achievement by investigating the associations between parenting styles and teaching strategies. The study showed that authoritative parents were better teachers, provided contingent support and used more frequently the child's "region of sensitivity to instruction".

Likewise, Mattanah, Pratt, Cowan, and Cowan (2005) explored the links between authoritative style, parental scaffolding behaviours during math tasks and children's academic achievement. The results suggested that parents who were characterised as affectionate, encouraging, and demanding and who promoted children's autonomy (dimensions of authoritative parenting) were more sensitive to provide the necessary assistance to their children according to their needs during a task. A more recent study by Carr and Pike (2012) also reported similar with the above results. The relation between parenting quality and maternal scaffolding practices during problem-solving was investigated using both cross-sectional and longitudinal data. In particular, the authors examined the links between positive and harsh parenting and parental contingent and non-contingent behaviours. Positive parenting referred to the "expression of affection and positive discipline" (p. 545), 
such as physical affection, being calm with the child. Harsh parenting referred to parent-child conflict and negative discipline such as "give a slap". The findings revealed positive links between positive parenting and mother's contingent scaffolding during problem-solving. Positive links were also found between harsh parenting and non-contingent scaffolding behaviours and negative correlations between harsh parenting and contingent scaffolding. The researchers commented that harsh parenting seems to elicit a particular and strong outcome on parental behaviours during parent-child interactions.

The relations between parenting styles and parental use of language have also been a subject of research. Pratt, Kerig, Cowan, and Cowan (1992) studied, among other relations, the links between parenting style and parental conversations with preschool children during play activities. The findings indicated that mothers with a more authoritarian parenting style used more directives and shorter utterances and less responsive language. Moreover, mothers with a more authoritative parenting style used fewer directives and more responsive speech. Therefore, the language style of authoritarian mothers is less possible to elicit conversations. One more study investigated, among other relations, the link between maternal teaching strategies about home safety and parental style in children between 2-2,5 years old and 3-3,5 years old (Morrongiello, Corbett, Lasenby, Johnston, \& McCourt, 2006). It was found that permissive parents used more explanations and fewer rules about this issue and that these strategies that were used by moderately and highly permissive parents, increased children's danger of medically-attended injury.

Parents' emotional competence goals have been found to mediate the relations between parenting styles and parental approaches (Chan, Bowes, \& Wyver, 2009). In particular, this study investigated the parental styles, parenting practices and emotion socialisation goals in Hong Kong - Chinese mothers. Parenting styles included an authoritative, an authoritarian and a psychologically controlling style, that is "the mothers' tendency to adopt psychological control as a parenting style" (p. 639). Parental approaches in response to children's emotions were categorised as coaching approach, emotion-encouraging approach and emotion-dismissing approach. The findings indicated that authoritative mothers implemented different approaches according to their emotional competence goals (individualistic or relational). Mothers with individualistic goals and an authoritative style adopted a coaching or an emotion-encouraging approach. On the other hand, parents with an authoritarian style and relational, emotional competence goals adopted a coaching and a dismissing approach to their children's emotional expressions. 
Overall, the above studies provide some evidence which underlines the positive relations between the authoritative parenting style and the parental contingent behaviours. It should be noticed that most of the above studies used an observational approach or interviews to assess parental authoritative parenting, considering behaviours such as autonomy support, demandingness, responsiveness. The present study investigated the relations between particular parenting styles (authoritative, authoritarian, permissive, strict) and parental supportive behaviours. Parenting styles were assessed in this thesis by mothers' self-reports (questionnaires). The findings will provide a better understanding of the way that the mothers of this study perceive their parenting style and whether their perceptions are related to their behaviours during mother-child interaction.

\subsubsection{Parental self-efficacy}

Another individual factor that is reported to affect parents' practices and behaviours is self-efficacy. "Parental self-efficacy is defined as beliefs or judgements about one's competence or ability to be successful in the parenting role" (Hess, Teti \& Hussey-Gardner, 2004). Coleman and Karraker (1997) mentioned that parental self-efficacy could be explained as the individual's perceptions about his/her ability to affect positively a child's behaviour or development. They also support that "according to the basic tenets of self-efficacy theory, as outlined by Bandura (1989), parental self-efficacy beliefs should incorporate both the level of specific knowledge pertaining to the behaviors involved in child rearing and the degree of confidence in one's ability to carry out the designated role behaviors" (p. 58). In this respect, in order for parents to feel competent in their role, they must be acquainted with the childcare behaviours, be confident that they can deal with them efficiently, believe that their children will react contingently and their social system will be helpful (Coleman \& Karraker, 1997). Ardelt and Eccles (2001) argued that parental self-efficacy (PSE) refers to the perceptions held by parents concerning their competence to facilitate their child's development by reacting appropriately to the child and the environment.

Previous studies have attempted to detect possible factors that are related to PSE. For example, children's difficult temperament has been positively associated with low levels of PSE (Porter \& Hsu, 2003). Maternal psychological distress has been associated with domain PSE (which includes broader parenting characteristics and behaviours), and it has also been supported that maternal role strain/overload is negatively related to PSE (Erdwins, Buffardi, 
Casper, \& O'Brien, 2001) while social support is positively correlated with PSE (Haslam, Pakenham, \& Smith, 2006).

Some studies supported a mediating role for PSE. In this frame, the study of Giallo, Treyvaud, Cooklin, and Wade (2013) showed that PSE mediates the link between parental distress (e.g., depression), children's difficult temperament and parental involvement. This study investigated factors that influence parents' participation in dyadic play activities. The results showed that parents who experienced psychological distress and who perceived their children's temperament as challenging were more possible to have negative beliefs about their abilities as parents, which influenced their participation in play activities. In addition, De Haan, Prinzie, and Deković (2009) in a prospective longitudinal study showed that both paternal and maternal parental competence (parents' beliefs of their ability in managing difficulties, daily demands, and controlling their child's behaviour) were significant mediators in the link between parental personality and parenting. Extraversion and agreeableness were associated with higher competence which in turn was negatively linked to over-reactivity. The direct links of these two parental personalities to parental warmth were stronger than the indirect link through parental competence.

Concerning the way PSE is related to some general parenting behaviours, studies report almost consistent results. For example, one study supported that mothers with high levels of PSE reported more positive behaviours towards their children (e.g., communication) than those with lower levels who reported more negative practices (e.g., inconsistent discipline, love withdrawal) (Hill \& Bush, 2001). The study of Shumow and Lomax (2002) using information from both adolescents and parents, suggested that PSE was a predictor of parental involvement and monitoring, which in turn encouraged children's academic and socio-emotional competence. Likewise, another study also found that PSE was positively associated with parental behaviours (e.g., encouragement, collaborative activities) that foster the academic achievement but mainly in Black mothers (Ardelt \& Eccles, 2001).

It seems that the way PSE relates to some parenting behaviour differs in mothers and fathers. This finding was mentioned by Murdock (2013) who examined parental self-efficacy in parents of children three to five years old. The study showed that for both mothers and fathers parental self-efficacy was positively linked to positive parental affect and negatively to negative affect but only for mothers. In addition, a positive correlation was found between parental self-efficacy and parental control but only for fathers, and a negative correlation between parental self-efficacy and hostile and coercive parenting but only for mothers. 
Finally, supportive or engaged parenting practices predicted paternal self-efficacy while hostile and coercive parenting predicted maternal self-efficacy.

The relations between PSE and parental involvement in children's education is another area of research. In this framework, PSE (e.g., parents' confidence in their ability to help with homework) is considered to be a key factor (Hoover-Dempsey, Battiato, Walker, Reed, DeJong, \& Jones, 2001) with high levels of PSE to be linked to increases in parental involvement (e.g., help with homework, educational activities with children, communication with the teacher etc.) (Hoover-Dempsey, Bassler, \& Brissie, 1992). In the same vein, Pelletier and Brent (2002) argued that parents who consider themselves to be more competent (e.g., beliefs about their teaching efficacy or their academic, social and motivational influence on the child) are more engaged in their pre-school children's education.

Longitudinal studies have tried to detect causal and reciprocal relations between PSE and parenting behaviours and supported that PSE stronger predicted high levels of positive controlling behaviours (monitoring and consistent discipline) over time as reported by Mexican American mothers and their adolescents than the other way around (Dumka, Gonzales, Wheeler, \& Millsap, 2010). Another study supported that parental sense of competence and parental behaviours were linked bidirectionally, indicating that parental sense of competence was a predictor of both parental supportive behaviours and low inept discipline, which in turn predicted later parental competence (Slagt, Dekovic, de Haan, Van Den Akker, \& Prinzie, 2012). A more recent study by Glatz and Buchanan (2015) explored the relations between three factors, that is, parental self-efficacy, parenting behaviours and externalising behaviours in adolescents. The study provided mainly evidence for a PSE driven process, but this was true only for mothers and during early adolescence. However, the results also showed relations emerging from parents and children during early to middle adolescence. For mothers, parental self-efficacy predicted children's externalising behaviours through parental reported promotive behaviours (parental involvement, positive parenting practices, such as rewards, compliments, kisses and discussion during punishment).

Taken together, these results suggest a general tendency for positive links between parental self-efficacy and parental warmth, affect, responsiveness and engagement. However, many of the above studies assessed parenting behaviours with the use of questionnaires or interview tools completed either by the adolescents, the parents or the examiners. Therefore, whether PSE affects the actual parenting behaviours observed during problem-solving contexts is still a question to be answered. Another important issue regarding PSE refers to the way it is assessed. Some studies above used domain-general assessments, other use 
domain-specific measures (where task-specific items are combined to one broader level) and some task-specific measures of PSE (referring to specific parenting tasks). The present study used a general measure of PSE to examine the relations between PSE and parental supportive behaviours during learning. Limitations regarding the assessment of PSE will also be reported in the Discussion chapter.

\subsubsection{Parental education}

Socio-economic status is one more factor that seems to affect parents' practices and behaviours. "Family socioeconomic status (SES) is an amorphous concept used in different ways by different social science disciplines" (Duncan \& Magnuson, 2003, p. 83). SES includes measures, such as family income, education and occupation (Braveman et al., 2005; Duncan \& Magnuson, 2003). As this thesis examines the links between maternal education and maternal supportive behaviours, the literature below will mainly focus on this specific aspect of SES.

Data from several studies suggest that parental education is positively linked to parental supportive behaviours. For instance, Carr and Pike (2012) supported that maternal education positively predicted contingent scaffolding behaviours through higher positive parental behaviours (affection, firmness and calmness, explaining and reasoning with the child) and lower harsh parenting. Similarly, Hoff-Ginsberg (1991) also supported that mothers from the upper-middle class group communicate with their children in a more contingent way than the mothers of the working class group.

Somewhat similar findings were reported by Thompson and Foster (2014) who examined parental metacognitive questions provided to preschool children during a collaborative task and two possible predictors of this kind of metacognitive support, namely family SES and stress in the parent-child relationship. Parent's educational level and occupation, as well as the stress in parent-child interaction, were linked to parental metacognitive questions after controlling for children's age and language ability. However, only parental education uniquely predicted parental metacognitive questions. Positive correlations between maternal education and maternal autonomy-supportive behaviours towards children have also been reported (Sullivan, McGrath, \& Harrison, 1999). One more research concluded in similar to the above findings (Mokrova et al., 2012). In particular, the study examined the relations between maternal values and quality of parenting (maternal cognitive stimulation and emotional support), family education and occupation (social status) 
and preschoolers' motivation (persistence). The findings of the study suggested that mothers with higher education and occupation backgrounds tended to value more their children's selfdirection and offered more cognitive stimulation and emotional support during challenging tasks. Moreover, it was found that family social status was directly and indirectly associated with parental supportive behaviours through the maternal values. It has also been reported in another study that parents from lower educational backgrounds supported more "didactic teaching methods" (p. 294), gave emphasis in performance and interacted with their children using less informal activities (e.g., reading to their children) (Stipek, Milburn, Clements, \& Daniels, 1992).

Economic difficulties could lead parents to be more hostile to their children (Conger, Ge, Elder, Lorenz, \& Simons, 1994). Shumow, Vandell, and Posner (1998) supported that even in a group of low-income families, parents who had the lowest income and education tended to use more harsh parenting behaviours. Zhang (2005) examined, among other factors, the relation between SES and parental promoting self-determination behaviours in their children. The findings showed that parents with higher education (e.g., college degrees) tended to encourage their children more often to discuss their interests, to make decisions, and set goals. Furthermore, the study indicated that parents who had higher income tended to encourage more their children's self-determination skills.

It seems that parents' differentiate their use of language according to who they address. This view was supported by Rowe (2008) who examined factors that mediate the relation between SES (education and income) and child-directed speech (e.g., amount of speech, vocabulary range) and they also tested the relation between SES and parents' language during discussions with the researcher and their toddlers. The findings showed no significant links between SES and parental speech used with the researcher. However, significant positive correlations were found between parental education and income and child-directed speech. Parental knowledge of child development mediated this relation. It seems that parents with higher SES (education and income) were more likely to have higher parental knowledge about children's development which in turn leads to more talking, greater use of a variety of words and fewer directive utterances. The researchers commented that these results might suggest that parental talk in general, is not affected by SES since there were no significant differences in their discussion with the researcher. Nevertheless, a previous study conducted by Hoff (2003) tested the hypothesis that the SES discrepancies (education and occupation) noticed in children's vocabulary could be attributed to their mothers' speech. The findings showed that the differences found between high SES and mid- 
SES children's vocabulary could be entirely attributed to children's maternal language (e.g., number and length of utterances, words and words types) which acted as a mediator in this relation.

Conversely, the study of Lowe, Erickson, MacLean, Schrader, and Fuller (2013) revealed no significant differences in maternal scaffolding behaviours (e.g., providing strategies, defining cause and effect) in relation to their educational level in children born with normal birth weight. This kind of association was reported only for the children that were born with very low birth weight, indicating that maternal education was linked to verbal scaffolding only for this group of children (Lowe et al., 2013; Lowe, Erickson, MacLean, Duvall, Ohls, \& Duncan, 2014). Lowe et al. (2013) explained that maybe these associations were not found in the normal birth weight children as the mothers in this group already employed more complex scaffolding. A further investigation on the subject was also suggested, including more socio-demographic information as it seems that parental education reflects "wider social and economic influences" (Lowe et al., 2014, p. 592).

The above findings provide insights into the relations between parental education and parental supportive behaviours. Although many of the above studies included in the same measure parental education, occupation and income, positive relations between parental education and parental supportive behaviours were in general supported. Few studies did not show such kind of associations. As suggested by Lowe et al. (2014), education is probably connected to both social and economic aspects of an individual's life. Nonetheless, further investigation is needed in this area regarding the way parents' education, as a distinct measure, is related to their behaviours during parent-child interaction in problem-solving contexts. Another issue that it should also be examined is whether parental education affects their behaviours consistently or during specific periods of a child's life and which particular parenting behaviours are more affective by to this demographic factor. It is possible that, in very young children, these links might not be so strong and the effects of parental education on parental behaviours during problem-solving to become more evident as children grow up.

\subsubsection{Nature of the task}

An interesting research topic is the way parents change their behaviours and attitudes towards their children as a function of the type or nature of the activity. From the ecological models' point of view, the nature of the task and its demands could affect parents' behaviour (O'Dell, 1995, as cited in Korat, 2009). In this respect, some studies explored the effect of 
different types of tasks on parental behaviours. For instance, Korat (2009) examined motherchild interaction during a book reading task and a family photo album task. Children came from Jewish Hebrew-speaking homes. In particularly, it was investigated the way that maternal talk changes according to the educational level of parents and the type of the activity. The findings indicated that the type of the activity influenced the way of maternal talking, that is, mothers used higher teaching levels in book reading compared to the other type of activity (looking at a family photo album). The author commented that these differences could be attributed to the nature of the book reading as it is an activity that enables more "distancing talk" (the extent to which parents go beyond the information) and "decontextualization" discussions.

Kermani and Janes (1999) investigated maternal scaffolding in immigrant Latino population during mother-child interaction in two types of tasks; the first was a home-like (play with homemade dough) and the second a school-like task (reading and looking at a book). The results of the study revealed that in the home-like tasks mothers were more responsive to their children's current stage of understanding, encouraged more their children's responses, used more modelling strategies, direct instruction and task orientation compared to the school-like task. As the researchers noticed, it is possible that both the familiarity with the home-like task as well as the freedom this task allowed concerning the choices and goals to account for this result. On the contrary, the school-like task may have directed mothers to employ more conventional strategies.

Similar findings emerged from one more study conducted by Aram (2002) who also investigated mother-child interaction during joint writing in Hebrew in two different tasks; one characterised as a task taking place at home (a name list for a birthday party) and a task taking place at school (writing words that were reading aloud). The results recognised differences in maternal behaviours during the two tasks. Specifically, in the first activity, the interaction was more pleasant, less pressured, affectionate and more supportive possibly because the mothers were feeling more free and confident while in the second activity, mothers were more directive and demanding.

It seems that parents change their behaviours according to the task demands. For instance, González (1996) attempted to provide an answer to the question about the effect of the type of the task on parent-child interaction. The tasks included the construction of a figure, reading a picture book and playing with household objects. Specifically, it was found that in the more structured tasks parents were more active maybe because children considered them as difficult. In a further ethnographic analysis of some parent-child interactions, it was 
found that parents used more commands in the construction task, more questions or declaratives in the reading task and more activity suggestions in the playing with household objects task. An interesting result emerged concerning parents' perception of their function in the task which further explained the above findings. Parents considered themselves to be "absolutely indispensable" in the construction task and "necessary interlocutors" in the reading task. They also believed that children must be more independent in the household object task.

Pellegrini, McGillicuddy-Delisi, Sigel, and Brody (1986) examined, among other relations, the effect of the task type and children's communicative status (language/communication disorder or not) on parent teaching strategies. The tasks included origami (paper folding) and book-reading tasks. The results showed that even though parents used more didactic strategies in the origami task, they also used some less demanding strategies in the same task, such as statements and conversational management. Highdemanding strategies were more employed in the book-reading task, such as questions. It is possible that high demanding strategies, such as questions are commonly adopted by parents during book reading because they enable them to turn their children's attention to the book. On the other hand, the use of both highly demanding and less demanding strategies in the origami task suggests that parents may use at the beginning more demanding strategies, but they lower their demands when the child needs help.

The relations between maternal autonomy support, maternal style and the nature of the task have also been studied. Grolnick, Gurland, DeCourcey, and Jacob (2002) explored these relations under two experimental conditions, a high pressure (emphasized the evaluation, performance and parental responsibility) and a low-pressure condition also taking into account previous children's ratings about maternal autonomy support, involvement and structure. The tasks involved a map and a poem task. The findings were different according to the type of the task. In the poem task, there were more verbally controlling behaviours elicited by mothers in the high-pressure condition, a result that stressed the importance of parental orientation in parent-child interaction (e.g., focusing on performance). In the map task, although there were no main effects, it was found that mothers with an existing controlling style who were assigned to the high-pressure condition were more controlling in their nonverbal behaviour. Explaining the differences in the two tasks, the authors suggested that for the verbal variables of the study there were more effects in the poem task as the latter was a more verbal task leading to more verbal interventions. Similarly, for the nonverbal variables, there were more effects in the map task. In addition, the map task elicited more 
controlling behaviours as it was a more structured task which may enable mothers to maintain their usual styles.

Mother-child conversations have been found to be affected by different settings, such as book reading, mealtime, dressing and toy play (Hoff-Ginsberg, 1991). This particular study showed that book reading elicited more "lexical diversity", "syntactic complexity" and "topic-continuing replies". In addition, interaction with toy play was more directive compared to the other settings, and it also contained the fewer conversation-eliciting utterances in contrast to mealtime and dressing which had the highest rates in this domain. The multifaceted differences noted in these settings imply that "supportive characteristics of child-directed speech do not vary as a single complex of features, but that different supportive features are maximized in different contexts" (p. 793).

In conclusion, the studies described above provide some evidence of how and why the nature of the task or context elicits different parental behaviours. This means that parents often employ different levels or qualities of parenting practices according to the task at hand. Most studies in the literature have overlooked this parameter when examining parentalsupportive behaviours during joint problem-solving. It is evident that the level of difficulty and the familiarity with a task differentiate parenting behaviours. Parental beliefs about the value of a task but also about its demands seem to affect the quantity and quality of parental supportive behaviours. Therefore, the present study will investigate which specific parental supportive behaviours, are employed when dealing with Visual-spatial and Language tasks. This kind of information is valuable for parents and teachers in order to be aware of how the context of a subject and task may affect their teaching strategies and behaviours towards students.

\subsection{Factors Related to Children's SRL and Achievement}

\subsubsection{Children's temperament and personality}

In the Section 2.3.1, the concept of temperament has been presented. Apart from the links between children's temperament and parental supportive behaviours during learning, a considerable literature has grown up around children's personality, temperament traits and children's learning skills or strategies. This thesis investigated three dimensions of children's temperament, which are Negative Affectivity, Surgency/ Extraversion and Effortful Control. Therefore, in this section, the studies which are closer to these three dimensions will be 
presented first, followed by studies with more general aspects of children's temperament and personality.

Concerning the relation between children's temperamental traits and their academic skills, such as language, reading and mathematics skills, relevant research has reported interesting results. For instance, the link between children's language abilities and temperament has been explored by Dixon and Smith (2000) who found in their first study that persistence, adaptability and positive mood at 13 months old children predicted language productivity at 20 months old when children demonstrated attention skills and positive emotionality. Concerning children's expressive and receptive language, the literature shows that children's positive affect at ten months old positively predicted expressive but not receptive language when children were 14 months old (Laake \& Bridgett, 2014). Other longitudinal studies found that children's temperamental dimensions, such as duration of orienting, smiling and laughter and soothability were positively associated with receptive vocabulary (Morales, Mundy, Delgado, Yale, Neal, \& Schwartz, 2000). Moreover, children from low SES backgrounds who were more likely to be readily stimulated and as a result were characterised as high in emotionality, demonstrated worse performance in their receptive vocabulary (Noel, Peterson, \& Jesso, 2008).

Considering children's effortful control, studies show positive links to language and arithmetic skills and academic achievement. For instance, the study of Blair and Razza (2007) explored the longitudinal relations between three to five years old children's executive function, effortful control, false belief understanding and children's math and literacy skills. The findings showed that teachers' reported effortful control and inhibitory control were positively associated with children's ability in math and letter awareness. Inhibitory control was the only factor that was independently related to mathematics knowledge, phonemic awareness, and letter knowledge. Liew, McTigue, Barrois, and Hughes (2008) discovered that first-grade children's adaptive/effortful control predicted their reading performance in third grade. Moreover, one more study mentioned that children's effortful control was significantly and positively associated with their grades (Valiente, Lemery-Chalfant, Swanson, \& Reiser, 2008). Zhou, Main, and Wang (2010) also argued that effortful control predicted GPA scores and this longitudinally study also revealed that this relation was slightly mediated by social competence, a result that was also supported by another study (Valiente et al., 2011). Deater-Deckard, Mullineaux, Petrill, and Thompson (2009) found a positive relation between effortful control and students' reading skills, but they did not discover any association with children's surgency (high-energy activation), except for 
moderating the effect of effortful control. In other words, the aforementioned positive relations were apparent if the children had lower levels of surgency. The positive links between children's attention skills and academic skills (e.g., vocabulary, reading and arithmetic) have also been mentioned in other studies (e.g., Garello, Viterbori, \& Usai, 2012; Usai, Garello, \& Viterbori, 2009; Welsh, Nix, Blair, Bierman, \& Nelson, 2010).

Similar conclusions emerged from one more study which explored the role of children's behavioural regulation (tapping mainly inhibitory control but also attention and working memory) in the prediction of children's academic skills (McClelland, Cameron, Connor, Farris, Jewkes, \& Morrison, 2007). In particular, this study found that children's behavioural regulation positively predicted children's skills in math, vocabulary and literacy in pre-kindergarten, controlling for children age, gender and language of assessment. Likewise, Espy et al. (2004) found that inhibitory control positively predicted preschool children's arithmetic skills even after controlling for the child's IQ, age, and maternal education.

In addition, gender differences in the relations between children's temperament and learning skills were detected by Davis and Carr (2002). These researchers explored the relations between first-grade children's temperament and their repertoire of strategies in mathematics as well as their ability to implement these strategies properly, taking into account children's gender. They found that boys who were less impulsive tried more to use retrieval strategies and they were more successful in using these strategies. On the contrary, boys who were more impulsive tended to employ more manipulatives (e.g., when children count using fingers or counters). Such relations were not found in girls. Specifically, girls who showed higher inhibition had low success in using retrieval strategies while they also demonstrated the same use of manipulatives compared to less impulsive girls. The study found no relations between girls' level of inhibition and the use of more cautious strategies, such as manipulatives, neither did it find relations between children's temperament and cognitive strategy use. The researchers commented that the young age of the participant children (first grade) possibly made more difficult the detection of such relations.

Finally, some studies turned their attention to the relation between children's delay of gratification and self-regulated learning. In this context, Bembenutty and Karabenick (1998) reported positive associations between delay of gratification and motivation, cognitive and metacognitive strategies in college students. Zhang, Karabenick, Maruno, and Lauermann (2011) depicted similar results, demonstrating associations between elementary Chinese children's motivation, SRL skills (cognitive, metacognitive, rehearsal, organisation) and 
delay of gratification. One more study, conducted by Maruno and Zhang (2010) aimed to determine the link between the academic delay of gratification and elementary Chinese children's motivation, SRL skills and performance. The researchers found positive associations between motivation, metacognition and academic delay of gratification. However, the other SRL skills, such as cognitive strategies, resource management as well as children's school performance were indirectly related to the academic delay of gratification through motivation and metacognition.

Studies have also investigated more general personality characteristics in children and their relation to children's SRL skills. In this context, it was suggested that children's conscientiousness was associated with SRL strategies, such as planning, setting a goal, time management, monitoring (Eilam, Zeidner, \& Aharon, 2009) while openness to experiences has been linked to meaning directed learning (e.g., SRL, critical processing, relating and structuring) and application directed learning [when students "try to employ what they learn to actual, real-world settings" (p. 1099)] (Busato, Prins, Elshout, \& Hamaker, 1999). Conscientiousness has also been reported to be positively linked to fact retention, synthetic and analytic learning, elaborative processing and methodical study in undergraduate college students (e.g., use of systematic study techniques) (Komarraju, Karau, Schmeck, \& Avdic, 2011). In the same study, Komarraju et al. (2011) found that Neuroticism was negatively associated with all the above factors while Openness and Conscientiousness predicted most of them. In the same vein, Bidjerano and Dai (2007) investigated the associations between specific SRL strategies and the Big Five personality dimensions in undergraduate students. The results showed that there were some consistent relations between specific strategies and personality traits. In particular, SRL strategies and skills, such as time management, effort regulation, elaboration, metacognition were positively associated with Consciousness and Intellect. In addition, it was supported that individuals who score high on Consciousness, Agreeableness and Emotional Stability and low on Intellect, tend to show more effective time and effort regulation skills even though they lack efficient critical thinking. The researchers commented that these personality types could offset students' difficulties met in challenging problem-solving situations.

The evidence of the above findings supports the idea that some of the children's temperament characteristics seem to be related to children's academic skills. Most of the above studies with similar dimensions of temperament to those used in the current thesis have investigated relations to students' language, reading and math skills. On the other hand, children's types of personality seem to have been investigated more frequently regarding 
their relation to children's SRL skills. Even so, the way that children's temperament is perceived by their mothers and its relation to children's employment of different aspects of SRL skills during online problem-solving has not attracted much research attention. The current thesis will investigate these associations, providing further data on this issue.

\subsubsection{Parenting styles}

In general, research regarding the impact of parenting styles on multiple aspects of children's development is ample. For instance, some studies have looked for associations between parenting styles and children's academic achievement, adjustment, competence, academic success (e.g., Lamborn, Mounts, Steinberg, \& Dornbusch, 1991; Steinberg, Lamborn, Darling, Mounts, \& Dornbusch 1994; Strage \& Brandt, 1999), motivation (Gonzalez \& Wolters, 2006) and child-mother attachment (Karavasilis, Doyle, \& Markiewicz, 2003).

Regarding parenting styles and students' learning and academic skills, the study of Erden and Uredi (2008) explored the way parenting styles, as perceived by $8^{\text {th }}$ grade students influence their SRL strategies and motivational beliefs (e.g., intrinsic value). The study revealed that, indeed, students' perceptions of parenting styles had an impact on different aspects of SRL (cognitive, metacognitive and motivational). Specifically, authoritative parenting was connected with the employment of more SRL strategies by students, in comparison to those whose parents had a more authoritarian, indulgent and neglectful style. Nonetheless, students who perceived their parents as indulgent used more cognitive and metacognitive strategies than students with authoritarian and neglectful parents.

Regarding children's academic achievement and competence, Aunola and Nurmi (2004) explored the effect of maternal psychological control ["refers to control attempts that intrude into the psychological and emotional development of the child" (Barber, 1996, p. 3296)] on their children's mathematical achievement from preschool to primary school. The results supported that maternal demonstration of higher psychological control was linked to slower progress in math and that maternal affection moderated this impact. Likewise, the study of Bronstein, Ginsburg, and Herrera (2005) revealed that higher levels of external parental control (e.g., demands, criticism), and low levels of guidance were related to $5^{\text {th }}$ grade students' poor achievement which in turn was a predictor of developing extrinsic motivation in $7^{\text {th }}$ grade. The opposite results appeared in the case of providing greater autonomy to children. Baumrind, Larzelere, and Owen's (2010) longitudinal study provided 
evidence for the long-term effects of parenting on children's competence. In particular, they found that the adolescents of authoritative parents when children were in preschool age were more competent and well adjusted compared to those of authoritarian parents. Berk and Spuhl (1995) examined the associations between maternal involvement in preschool children's private speech and task performance. The findings of the study revealed that the authoritative parenting style was a predictor of children's private speech and performance.

Concerning the relations between parenting styles and children's motivation, Leung and Kwan (1998) found that children's perceived authoritative parenting style was connected to Chinese children's intrinsic motivation and neglectful parenting to amotivation, while authoritarian parenting was related to extrinsic motivation and amotivation. Similarly, Gonzalez and Wolters (2006) supported that students' perceived authoritative parenting style was positively linked to students adaptive motivational beliefs (e.g., intrinsic motivation) and autonomy, while permissive parenting style was linked to less adaptive motivational beliefs and performance goal orientation. Authoritarian parenting style was positively linked to performance approach goals. Consistent with the above findings, the study of Boon (2007) also supported that authoritative parenting (as assessed by adolescents) was related to greater students' academic achievement, self-efficacy and mastery goals and neglectful parenting style was associated with low academic achievement and self-efficacy. Aunola, Stattin, and Nurmi (2000) investigated the relation between parenting styles and adolescents' achievement strategies. The findings revealed that authoritative parenting style was positively related to adolescents' adaptive and task-oriented achievement strategies as well as with low failure expectations levels and low passivity. On the other hand, the neglectful style was positively associated with dysfunctional strategies (e.g., task-irrelevant behaviours) and passivity. Finally, the authoritarian and permissive style was positively related to passive behaviour.

Together the above findings suggest that there is a tendency for a positive link between authoritative parenting style and student's learning skills. However, this association is related to the different methodologies used to assess parenting styles, such as adolescents' self-reports, parents' self-reports, observation or a combination of both. These methodological approaches differ from the one followed in this study in which parenting styles were assessed by parents' self-reports (given the young age of the children). Moreover, in the present study children's SRL skills were observed during problem-solving, unlike previous studies that have used different tools (e.g., questionnaires). This area under 
examination will provide information regarding the way that the mothers of our study perceive their parenting style and whether this is linked to children's SRL skills.

\subsubsection{Parental self-efficacy}

The literature concerning the relations between parental self-efficacy and children's SRL skills needs further documentation. There are few studies that have tried to identify such kind of relations both directly and indirectly, including more general aspects of children's learning behaviours. For example, Coleman and Karraker (2003) explored the relation between maternal self-efficacy, maternal competence, children's behaviour and children's

performance on the Mental Scale of the Bayley Scales of Infant Development. Parenting competence was positively related to children's performance and adaptive behaviours. Moreover, domain-specific parenting self-efficacy beliefs positively predicted children's performance and some of the children's behaviours (affection, avoidance, enthusiasm, negativity).

Factors such as maternal self-efficacy, older age and higher education may act as protective factors for children with difficult individual traits (e.g., neonatal threats) from demonstrating regulation problems, and should be taking into consideration for intervention programs (Jusiene, Breidokiene, \& Pakalniskiene, 2015). The study of Lynch (2002) proposed that maternal self-efficacy about helping their children's reading achievement was positively associated with children's self-beliefs as readers but not with their reading achievement. A longitudinal study that examined the psychosocial outcomes in children's lives in relation to PSE levels found that parents with high PSE had adolescents who reported higher self-efficacy in handling learning tasks, fewer depressive signs, better communication with parents, and fewer behavioural difficulties (Steca, Bassi, Caprara, \& Delle Fave, 2011). The same study supported that these adolescents showed higher concentration, engagement and motivation in academic tasks as well as more happiness and freedom in their family interactions.

Furthermore, Coleman, Trent, Bryan, King, Rogers, and Nazir (2002) investigated the extent to which mothers' "competence promoting behaviours" (e.g., constructive assistance, sensitivity) and mothers" "competence inhibiting behaviours" (e.g., negative emotionality, controlling behaviours, nervousness) were associated with their toddlers' performance on the Bayley scale and the role of PSE in this relation. The findings showed that PSE was a mediator between overall parental "competence inhibiting behaviours" and 
children's performance on the Bayley Mental Scale as well as between the parenting behaviour described as "overlook of child's misbehaviour" and children's performance on the Bayley Mental Scale. Ardelt and Eccles (2001) examined the impact of PSE on children's self-efficacy and academic achievement in different family environments. The findings revealed that PSE had a stronger effect on children's achievement in black families than in white families and this effect was both direct and indirect through parental promotive strategies and children's self-efficacy. The study of Shumow and Lomax (2002) resulted in a model of paths between neighbourhood quality, PSE, parental behaviours and academic and socio-emotional competence of adolescents. The findings supported that high neighbourhood quality positively predicted PSE controlling for SES, which then predicted parental involvement and monitoring (controlling for environmental circumstances), which in turn predicted adolescents' academic and socio-emotional adjustment. The study of Purdie, Carroll, and Roche (2004) investigated the predictive role of parental efficacy in academic and non-academic self-regulation. The results revealed that parental efficacy predicted parental involvement (e.g., be loving, responsive and involved) which in turn predicted academic (goal setting or planning, strategies for the task, self-motivation, monitoring, and evaluation) and non-academic self-regulation in adolescents. Finally, Bandura, Barbaranelli, Caprara, and Pastorelli (1996) found, among other results, that maternal self-beliefs about their efficiency to encourage their children's academic development have a positive impact on children's achievement through the academic aspirations they have for their children.

In conclusion, the above studies report both positive direct and indirect links between parental self-efficacy and some general aspects of children's learning. However, very limited information is available regarding the relations between PSE and children's SRL skills. Moreover, the associations found (or not) between PSE and children's learning and achievement are affected by the measures of PSE and by the level of its assessment (i.e., general, domain, task-specific) but also from the role of PSE in mediation paths (either as a mediator, or predictor).

\subsubsection{Parental education}

Various aspects of family SES and parental education levels have been investigated in the literature. There is a body of research that associated the family SES and education levels with children's development, such as their cognitive achievement, language skills and behaviour (Hoff \& Tian, 2005; Magnuson \& Duncan, 2006; Yeung, Linver, \& Brooks-Gunn, 
2002), in favour of children from families with higher SES. In this respect, researchers draw their attention in examining the effect of maternal education on children's language skills. For example, a longitudinal study by Dearing, McCartney, Weiss, Kreider and Simpkins (2004) examined maternal educational level as a moderator in the relationship between maternal involvement at kindergarten and children's achievement in literacy. Children's performance in fifth grade was positively associated with maternal involvement, mainly for children with less educated mothers. Moreover, children's performance did not differ with respect to maternal education if mothers were highly involved. The researchers pointed out that interventions in low SES families should increase parental participation in order to encourage children's feelings and performance in literacy.

The impact of maternal education on maternal teaching talk and 5-6 years old children's developing literacy was investigated by Korat (2009). Children's literacy skills were positively associated with high maternal educational background possibly because mothers from higher education backgrounds had higher teaching talk levels in their activities. In the same vein, Raviv, Kessenich, and Morrison (2004) studied the way SES (education and income) affects three years old children's language skills. This study found that parental factors (maternal sensitivity and cognitive stimulation) partially mediated the relation between SES and children's verbal understanding, expressive language skills and receptive language skills. One more study examined the productive vocabulary of two years old children from different SES (education and occupation) and their learning experiences at two times (Hoff, 2003). Children from higher SES backgrounds had expanded their vocabulary more compared to the rest of the children, and maternal speech acted as a mediator in this relation. Another study revealed a direct relation between SES and children's emergent literacy but also a mediational model was supported in which home learning experiences (e.g., reading activities) acted as mediators in the relationship between SES (education and income) and children's developing literacy skills and social functioning (Foster, Lambert, Abbott-Shim, McCarty, \& Franze, 2005).

Regarding children's cognitive, metacognitive and motivational skills, the literature reports positive links to SES. An interesting study was carried out by Pappas, Ginsburg, and Jiang (2003). Specifically, the impact of SES (assessed mainly by the type of school the children attended) on about 4 to 6 years old children's metacognitive skills (e.g., mistake recognition, adaptability, awareness and expression of thinking) and related language in mathematical type problems were investigated. The findings showed that in general, children did not differ significantly in their metacognitive skills and the amount and type of speech. 
However, children from the upper SES backgrounds demonstrated better skills in expressing their thinking and in offering explanations. Roberts, Bornstein, Slater, and Barrett (1999) explored the link between young children's cognitive development at 9, 12, 15, 18, and 27 months and parental education level. Their results showed that even in very young children, parental education was significantly correlated with several but different measures of children's cognitive development. Other studies provided evidence that parental responsivity, enrichment (e.g., resources to enhance children's development) and family companionship (e.g., the parental participation in child activities) mediated the relation between SES (e.g., education, occupation and income) and children's inhibitory control and working memory (Sarsour, Sheridan, Jutte, Nuru-Jeter, Hinshaw, \& Boyce, 2011). Mokrova et al. (2012) suggested that children from higher social status backgrounds (education and occupation) and whose mothers valued children's self-direction were more persistent in the face of difficulties during problem-solving and that maternal emotional support mediated these associations. The authors commented that maybe children at this age (preschoolers) are based more on their mothers' emotions and less on the quality of information the mothers provide in order to consider the task as worthy to try. In other words, the researchers supported that "it may be the case that motivational development begins at the emotional level whereas cognitive aspects of motivation become important later in childhood" (p. 11).

Data on the relations between children's executive function and parental SES have also been reported. For example, Ardila, Rosselli, Matute, and Guajardo (2005) investigated the relation between 5-14 years old children's executive function, type of school (private or public) and parental education. The researchers supported that parental education significantly and positively correlated with children's executive function measures. The work of Rhoades, Greenberg, Lanza, and Blair (2011) showed that early socioeconomic risk (e.g., exposure to poverty, single-parent families) was significantly associated with 36 months old children's executive function skills and that this link was explained in some cases by parenting behaviours. The work of Noble, Norman, and Farah (2005) went further exploring the way SES (education, occupation and income) is related to specific brain systems or cognitive functions. The study revealed that differences in SES were linked to discrepancies in kindergarten children's performance in language systems (large effect size) and executive function systems (moderate large effect size), followed by fewer differences in visual cognition, visual-spatial skills and memory. The researchers argued that the differences in the magnitude of the impact regarding the relations between SES and cognitive performance for the language and executive function might be due to the lengthened maturation of the 
language and executive function systems that made them vulnerable in possible environmental diversity. Then again, as the researchers mention, "different systems may be differentially reliant on the types of enculturation processes that vary across SES" (p. 84).

Concerning the relations between children's academic achievement and parental SES, the findings are consistent. DeGarmo, Forgatch, and Martinez (1999) tested a mediational model exploring the differential impact of divorced mothers' education, occupation and income on academic outcomes for boys between 6-9 years old. The study showed that maternal education, income and occupation attainment positively predicted the quality of parenting which in turn had an indirect impact on boy's achievement through home activities that enhance children's skills and through adaptive school behaviour. The study also found that the relation between maternal education and occupation also had direct links to children's achievement. In addition, home skill-building activities accounted for the relation between maternal education and children's achievement. The relation between maternal occupation and children's achievement was partially mediated by home activity and school behaviour. Considine and Zappalà (2002) with a sample of almost 3000 students from economically disadvantaged environments examined the effect of socioeconomic level, family, individual, and background factors on children's school performance. The findings highlighted the significant role of parental education on children's academic achievement while the results also show that family income was not a significant predictor of children's performance when controlling for other factors. The researchers supported that the social (e.g., parental education) and the economic elements of SES have a different impact on school achievement, and thus their unique contributions to children's various aspects of development should be explored. Davis-Kean (2005) investigated the indirect path between parental education and income, parental beliefs and behaviours (e.g., play, educational expectations) and children's academic achievement. The results showed that parental education and income had an indirect effect on children's achievement through parental achievement beliefs, the warmth in parent-child interactions and the stimulating home behaviours for African American families while parental education had a direct and indirect effect on children's achievement for the European American sample.

Regarding mathematics, a study conducted by DeFlorio and Beliakoff (2015) investigated SES and children's mathematical development during preschool age as well as parental beliefs about mathematical development and their link to children's existent knowledge. The findings showed that children from middle SES backgrounds had broader mathematical knowledge than children from lower SES backgrounds. In addition, pre- 
kindergarten children from low SES had similar mathematical knowledge to the one of younger children in preschool entry year from middle SES, meaning that these prekindergarten children were "one developmental year behind" the latter. SES discrepancies in the type of math support in the home were also detected. Parents from middle SES participated more often in activities that encourage mathematical skills and used approaches that implemented the use of math skills in daily activities. Moreover, children from middle SES participated in a wider variety of activities weekly.

In general, there are differences in the conceptualization of SES in literature, as it is measured via various indices from study to study. When parental education is measured, then both direct and indirect effects of parental education are observed. Parental education is related to some aspects of children's development and not to other. So, conclusive comments cannot be reached. What is more, some of the studies above have found relations to specific children's skills but not to other, and in some cases, these relations changed over time. Together these studies probably suggest that children's skills may be differentially associated with parental education and that children's age may perhaps play a significant role in these relations. Hence, the present study will explore the link between maternal education and children's different SRL skills, in two cognitive domains and at two different time points, to provide a better understanding of these relations.

\subsubsection{Nature of the task}

Research addressing the role of the nature or type of the task on children's SRL skills is limited. However, the few studies that investigated this area report that the context and the nature of the task seem to elicit different children's behaviours. In this context, Wolters and Pintrich (1998) investigated contextual differences in students' motivation and SRL in math, English and social studies, in $7^{\text {th }}$ and $8^{\text {th }}$ grade. In particular, they included measures of student's cognitive strategy use, regulatory strategy use, self-efficacy, task value, motivational and performance. The findings revealed that the motivational aspects of SRL (reported value, interest, self-efficacy) were to some degree context specific. Mathematics was viewed as more important, interesting and useful compared to the other two subjects. Moreover, children mentioned higher levels of cognitive use in the social studies, then in English and last in mathematics. No differences were found in regulatory strategy use.

One more study investigated SRL in different disciplines (humanities, social science and natural science courses) in college students (Vanderstoep, Pintrich, \& Fagerlin, 1996). 
The findings revealed that performance level was significantly dependent on motivation and strategy use mainly in natural science courses, then in social science courses and much less in humanities. Cognitive strategies were more significant in the natural and social courses. It seemed that the nature of the test exams (a multiple-choice in natural and social sciences compared to essay tests or papers in humanities courses) might explain this difference. On the other hand, performance at the end of the term was positively linked to task value (including interest, importance and utility of the course material), only in the natural and social science courses.

Likewise, Rotgans and Schmidt (2009) also explored SRL in different contexts. Specifically, first-year students completed a general version of the MSLQ (Motivated Strategies for Learning Questionnaire) before entering the Polytechnic and a course-specific version at the end of the first semester for science, mathematics and English courses. The findings, in general, supported that the underlying factorial structure of SRL is not contextdependent at least for the cognitive component. However, significant latent mean differences were detected between mathematics/science and English, with lower scores for English in the measures of metacognitive self-regulation, effort regulation, elaboration and task value beliefs and lower scores for mathematics in the self-efficacy judgments.

The motivational patterns demonstrated by primary school students regarding their values in math, reading and writing and whether these patterns were linked to academic performance and self-concept of ability were investigated by Nurmi and Aunola (2005). Four groups emerged from the analysis including those who highly value the three subjects (math, reading and writing), those with a high motivation in math, those who had low interest in math and those who had low interest in reading and writing. This differentiation suggested that even young students value the various school subjects differently.

The familiarity and specificity of the task also account for the discrepancies noticed in different tasks. For example, Kermani and Janes (1999) studied mother-child interaction in Latino families across a home-like and school task. The home-like task included "a creative play with homemade dough" (p. 140) and the school type task included a picture book to read and look. Children's behaviours included initiation (e.g., asking questions, making comments) and "active engagement with the task and social partner" (p. 142). The findings revealed that children were more actively engaged in the home-like task probably because this task was more specific.

The above studies outline that the nature of the task should be taken into consideration when studying students' learning in different contexts. In general, the limited research in this 
field suggests that students and young children value differently their school courses and employ different levels of strategies according to the task at hand. It seems that both the nature of the task as well as its familiarity and specificity explain some of the discrepancies. However, more studies are needed to examine the way different tasks affect various aspects of SRL skills in young children. This thesis will look into these relations to present information about whether young children employ different levels of SRL skills in Visualspatial and Language tasks. This information is critical for teachers and parents in order to facilitate learning in different cognitive domains.

\subsection{Rationale and Significance of the Study. Aims and Hypotheses of the Study.}

The literature presented in the previous units underlined the significance of children's SRL skills as well as the prominent role of parental supportive behaviours in fostering children's development of SRL. In general, the studies described in the previous sections tend to report positive relations between aspects of parental supportive behaviours, children's learning skills and academic achievement. These links were more consistently mentioned in the case of parental contingent behaviours and autonomy support. At the same time, research in this field underlines the multifaceted nature of parental supportive behaviours. Different methodological approaches (e.g., observational, teachers' or students' self-report, interviews) and conceptualizations of this term are regularly employed which complicates the deep understanding of which specific aspects of parental behaviours are associated with children's distinct SRL skills. Regarding the role of children's and parents' characteristics in these relations, complex networks of relations appeared. The literature indicated both bidirectional and unidirectional relations between parental and children's characteristics (children's temperament, authoritative parenting, parental education, parental self-efficacy), a variety of parenting practices and children's skills. Some studies reported that these attributes might act as mediators or their effect on parents' and children's behaviours may be indirect through other variables (e.g., home learning conditions).

To date, studies have approached the role of parental (i.e., mostly maternal) supportive behaviours in children's SRL skills by examining mainly the socio-emotional aspects of parent-child interaction. Other aspects of parental support, such as the metacognitive support, are so far overlooked. In addition, most research has not explored these links in actual problem-solving contexts and even fewer examined SRL skills in young children. Investigating the actual parental support as it unfolds in authentic problem-solving 
situations might shed further light on what contributes to the development of children's SRL skills and consequent achievement in real life. As past studies document the importance of the kind of parental support for children's developing abilities, learning skills, and academic achievements, further data are needed to understand the multiple dimensions of the supportive parenting behaviours and their role in the various aspects of children's learning skills. Moreover, far too little attention has been given to addressing these relations longitudinally, investigating how the relations between parental behaviours and children's SRL skills develop over time. The examination of these relations longitudinally can contribute to our knowledge of children's developing self-regulation skills at early ages and on the way adults can better support children during learning and achievement efforts.

Taking the above into account, the present study aims to provide insights on the dynamics of the mother-child interaction during actual problem-solving situations and on the links between maternal supportive behaviours and children's developing SRL skills and cognitive performance. The main objective of the study is to explore at the same time multiple dimensions of parental support of learning and their relations with specific SRL skills in young children. This study adopts a micro-analytic methodology to capture the above behaviours in actual situations. Moreover, the relations between maternal supportive behaviours, children's SRL skills and performance are investigated longitudinally, examining one to one dynamic interactions and the way these relations develop over time. Finally, this study also seeks to offer data concerning parents' and children's characteristics that might affect the above relations such as children's temperament, children's age and gender, maternal education, parenting style and parental self-efficacy. This kind of information is crucial for interventions focusing on encouraging positive parent-child interactions that foster children's skills to control and regulate their cognition and motivation during learning. Finally, another objective of this research was to explore whether mothers differentiate their level of support and whether children employ higher or lower levels of SRL skills, according to the nature of the task at hand.

Therefore, this study is expected to make an original contribution both theoretically and methodologically. From a more theoretical view, the study will offer insights into the links between students' SRL skills, maternal behaviours and maternal and children's characteristics that act possibly in combination early in children's learning course. Methodologically, this study will adapt Neitzel and Stright's (2003) observation system for parents to assess maternal supportive behaviours. This tool is a helpful measure for recording the actual on-line adult-child interactions focusing on behaviours that encourage SRL skills. 
Moreover, the present study will undertake a micro-level analysis and a longitudinal analysis, promoting the advancement of our knowledge on how these maternal and children's behaviour develop during problem-solving situations and how they change in time. Regarding the practical implications, this study will provide valuable information for the development of intervention programs with the aim of educating parents and educators about constructive behaviours that support the development of children's learning skills and the encouragement of more positive interactions with the child.

Based on previous studies, the research hypotheses of the thesis were formed as follows:

\section{Demographic variables and their relations to maternal supportive behaviours, children's SRL skills and cognitive performance}

$\mathrm{H}_{1 \mathrm{a}}$. It was expected that maternal education and children's age should be significantly and positively correlated with maternal supportive and contingent behaviours.

$\mathrm{H}_{1 \mathrm{~b}}$. It was hypothesized that maternal education and children's age should be significantly and positively correlated with children's SRL skills.

$\mathrm{H}_{1 \mathrm{c}}$. It was hypothesized that maternal education and children's age should be significantly and positively correlated with children's cognitive performance.

\section{Relations between children's SRL skills and cognitive performance}

$\mathrm{H}_{2}$. It was expected that children's SRL skills (Cognitive-Metacognitive, EmotionalMotivational, Autonomy skills) should be significantly and positively linked to cognitive performance at both research times and tasks. We also assumed that children's Cognitive and Metacognitive skills should have the strongest correlations with cognitive performance, as previous studies suggest.

Maternal supportive and contingent behaviours and their relations to children's SRL skills and cognitive performance at Time 1 and Time 2

$\mathrm{H}_{3 \mathrm{a}}$. It was hypothesised that maternal Contingency, maternal Cognitive and Metacognitive support, maternal Emotional and Motivational support and maternal Autonomy support should be significantly and positively related to children's Cognitive and Metacognitive skills.

$\mathrm{H}_{3 \mathrm{~b}}$. It was hypothesised that maternal Contingency, maternal Cognitive and Metacognitive support, maternal Emotional and Motivational support and maternal 
Autonomy support should be significantly and positively linked to children's Emotional and Motivational skills.

$\mathrm{H}_{3 c}$. It was expected that maternal Contingency, maternal Cognitive and Metacognitive support, maternal Emotional and Motivational support and maternal Autonomy support should be significantly and positively linked to children's Autonomy skills.

$\mathrm{H}_{3 \mathrm{~d}}$. It was expected that maternal Contingency, maternal Cognitive and Metacognitive support, maternal Emotional and Motivational support and maternal Autonomy support should be significantly and positively linked to children's cognitive performance.

\section{Relations between maternal parenting style and maternal supportive behaviours, children's SRL skills and performance}

$\mathrm{H}_{4 a}$. It was hypothesised that maternal authoritative parenting should be significantly and positively linked to maternal supportive and contingent behaviours.

$\mathrm{H}_{4 \mathrm{~b}}$. It was hypothesised that maternal authoritative parenting should be significantly and positively linked to children's SRL skills and cognitive performance.

\section{Relations between maternal self-efficacy and maternal supportive behaviours, children's SRL skills and performance}

$\mathrm{H}_{5 \mathrm{a}}$. It was expected that maternal self-efficacy should be significantly and positively linked to maternal supportive and contingent behaviours.

$\mathrm{H}_{5 b}$. It was hypothesised that maternal self-efficacy should be significantly and positively linked to children's SRL skills and cognitive performance.

\section{Relations between children's temperament and maternal supportive behaviours, children's SRL skills and performance}

$\mathrm{H}_{6 \mathrm{a}}$. It was expected that children' effortful control should be significantly and positively linked to maternal supportive and contingent behaviours.

$\mathrm{H}_{6 \mathrm{~b}}$. It was hypothesised that children's effortful control should be significantly and positively linked to children's SRL skills and performance. 


\section{Longitudinal associations between children's SRL skills at Time 1 (T1) and children's}

SRL skills and cognitive performance at Time 2 (T2)

$\mathrm{H}_{7 \mathrm{a}}$. It was expected that children's SRL skills at Time 1 should significantly predict part of the variance of children's SRL skills at Time 2 in both cognitive domains.

$\mathrm{H}_{7 \mathrm{~b}}$. It was expected that children's SRL skills at Time 1 should significantly predict part of the variance of children's cognitive performance at Time 2 in both cognitive domains.

Longitudinal associations between maternal supportive and contingent behaviours at Time 1, and children's SRL skills and cognitive performance at Time 2.

$\mathrm{H}_{8 \mathrm{a}}$. It was hypothesized that maternal supportive and contingent behaviours at Time 1 should significantly predict part of the variance of children's SRL skills at Time 2 in both cognitive domains.

$\mathrm{H}_{8 \mathrm{~b}}$. It was hypothesized that maternal supportive and contingent behaviours at Time 1 should significantly predict part of the variance of children's cognitive performance at Time 2 in both cognitive domains.

\section{The effects of the nature of the task on the study's variables.}

$\mathrm{H}_{9}$. It was expected that the nature of the task should differentiate the level of maternal supportive and contingent behaviours and the employment of children's SRL skills. No specific hypotheses were made as different types of tasks, different parental behaviours and children's skills are assessed in literature and, therefore, there are no consistent data. Moreover, although it seems plausible to assume that the relations between the study variables will be differentiated as a function of the cognitive domain examined, the lack of previous data does not support such a statement. 


\section{Chapter 3 Methodology}

This chapter presents the methodology underpinning this dissertation in order to answer the research questions. It also describes the two pilot studies undertaken to test the appropriateness of the measurements, questionnaires and tasks as well as the procedures followed during the two phases of the main study. At the end of this chapter, validity and reliability issues and the ethical considerations are also discussed.

\subsection{First Pilot Study - CBQ-VSF}

The first pilot study examined the suitability of the study's questionnaires. In particular, one of the three questionnaires used in this study had not been previously translated into the Greek language. Therefore, Children's Behaviour Questionnaire Very Short Form (Putnam \& Rothbart, 2006) was translated into the Greek language and afterwards a back translation was implemented by an English teacher. The two English versions were compared to each other and changes were made in the statements $2,8,27,28$, 32 and 33. The questionnaire was then administered to six mothers from the city of Volos to test its content validity. Based on the mothers' comments some improvements were further made to the statements of the measurement scale and the items 4, 6, 7, 15, 18, 19, 31 and 36 .

\subsection{Second Pilot Study}

A second pilot study was conducted to test the appropriateness of the tasks, measures and the scoring criteria of the observation forms. This step was necessary to decide what kind of tasks should be used in the mother-child problem-solving condition and the child-alone condition. This pilot study could also detect potential inaccuracies and problems in the scoring criteria used to assess the maternal supportive behaviours and children's SRL strategies.

\subsubsection{Participants}

Nine mother-child dyads took part in the second pilot study. There were five boys and four girls between 48-72 months old (mean age $=64$ months). Mothers were between 30 to 49 years old, and all of them had a university or/and postgraduate degrees. The participants came from a medium-sized town in central Greece (Volos, Karditsa). 


\subsubsection{Measures}

Cognitive tasks. Each child was given two types of tasks to solve alone, that is, Visual-spatial and Language tasks. Analogous tasks were also administered to be jointly solved by the mother-child pair to assess maternal supportive behaviours after the child-alone problem-solving. The cognitive tasks will be described in detail below in the Main study section.

Visual-spatial tasks. Thirty coloured cubes were given to the children. They were then asked to replicate three different patterns of cubes assemblies as they were depicted in relevant card-models. Children had been previously practising with the researcher in four examples. Next, three new tasks of cubes assembly were given to each mother-child pair. The examiner informed the mother to feel free to support her child when necessary to complete the tasks.

Language tasks. For this task, six cards illustrating heroes (three cards), objects (one card), and places (two cards) were given to each child. In this task, children had to develop a story based on these cards. Three different criteria were used to assess children's performance: Logical thinking and coherence of meanings (e.g., connection, logical sequence of meanings); Vocabulary (e.g., children's verbal fluency, reporting all of the information showed in the pictures, the length of the sentences), and Structure of the story (i.e., the story has distinct parts, such as a beginning, a middle and a closure part). After that, each motherchild pair was asked to develop a story using the six new cards given to them, depicting again heroes, objects and places. At the outset, the mother had to appraise the cards and then choose the cards she needed to start narrating a spontaneous story. Then, the child had to continue and finish the story using the rest of the cards. The mother could help the child when required. The instructions given in these tasks were the same as those in the main study, and therefore, are described in the relevant section.

Children's strategic behaviours. For the assessment of children's actual strategic behaviours during problem-solving that are indicative of SRL, the Strategic Behaviour Observation Scale (SBOS) was used (Dermitzaki, Leondari, \& Goudas, 2009). Six children's strategies were assessed in this study during problem-solving including Cognitive, Metacognitive, Motivational/Volitional and Autonomy strategies. This structured observation tool uses a 4-point scale to evaluate children's actual SRL skills during problem-solving. SBOS is described in more detail in the Main Method section. 
Maternal supportive behaviours. To assess mothers' supportive behaviours during joint problem-solving, we used Neitzel and Stright's (2003) observational coding system. This system was implemented in the current pilot study to investigate if it is an appropriate coding scheme for the aims of this particular study. According to Neitzel and Stright (2003), this observation system focuses on three aspects of parental support. Cognitive Support is comprised of (a) Metacognitive Information, the degree that the parent provides metacognitive information to the child in a way that reveals the thinking process of the problem; and by (b) Manner of Instruction, the degree that parents provide directions in small discrete steps (Task Difficulty Regulation) as well as the degree to which the parent summarizes the steps of the tasks and discusses the progress in relation to the ultimate goal of the project (Review). Emotional Support includes an assessment for (a) Encouragement, the degree to which the parent provides comfort and support verbally or non-verbally, and an assessment for (b) Rejection, the degree to which the parent provides redirection in a negative way, criticism, disapproval or disgust, dismissal of the child's efforts, nonverbal gestures of non-support, or other negative or inappropriate reactions to the child. Finally, Transfer of Responsibility is assessed by (a) Parents' Over-controlling, the degree to which the parent exercises control over the child's problem-solving attempts, beyond what appears necessary for the child to complete the task; and (b) Parents' Encouragement of Active Involvement, which refers to the degree to which the parent encourages the child's active cognitive involvement in the problem-solving task. Maternal behaviours were rated on a 5-point scale ranging from 1 (low) to 5 (high), as suggested by Neitzel and Stright (2003).

\subsubsection{Procedure}

The mothers and the children were recruited from state kindergartens and participated voluntarily in the study. In the task-alone condition, the child was first asked to try and solve alone the cognitive tasks. In the mother-child condition, the child had to solve the tasks under the direction and the support of the mother. A voice recorder was used to record each meeting with the permission of the mother. The examination lasted about one hour.

\subsubsection{Changes in the measures after the pilot study}

Cognitive tasks. After the pilot administration of the cognitive tasks, some concerns aroused about the level of difficulty of the Visual-spatial tasks.

Visual-spatial tasks. Regarding the Visual-spatial tasks, it was decided to replace the 
pilot study's tasks with four tasks of gradually increasing difficulty that better corresponded the various levels of children's abilities.

Mother-child tasks. Changes and additions to the Visual-spatial tasks were necessary as many of the initially selected tasks were easy for the children, and, consequently, there was no need for the mother to provide assistance. Hence, regarding the mother-child Visualspatial tasks, new more challenging tasks of gradually increasing difficulty were introduced following the respective changes in the individual tasks. The total number of tasks given to the mother-child pair was four. One extra difficult task was given to the mother-child pair in case that the previous four were relatively easy for the child leading to less maternal supportive behaviours.

The maternal observation system. The pilot study pointed out the need for a better conceptually clarification of the maternal behaviours under observation. Moreover, the observation criteria needed to be more clear and precise. Moreover, a specific endeavour of this study was to match the maternal supportive behaviours assessed with the respective children's strategic behaviours. Hence, modifications and further elaborations proceeded regarding the maternal behaviours and the assessment criteria. These changes were based on previous research on children's SRL and to previous tools for assessing SRL skills (e.g., SBOS).

In particular, "Cognitive support" in the original Neitzel and Stright's (2003) observation system, included both "Metacognitive information" and "Manner of instruction". In the revised observation system, maternal "Cognitive support" formed a distinct category. "Metacognitive support" formed a second distinct group including three maternal behaviours which are considered to activate children's metacognition. Elements of the "Manner of instruction" were incorporated within the Metacognitive supportive behaviours (see the Main study section). Moreover, the original aspect of "Emotional support" changed into "Emotional and motivational support", and it was also referred to the motivational climate of the mother-child interaction. Finally, the original "Transfer of responsibility" was renamed as "Autonomy support" respectively. Regarding the scoring scale of the revised observation system for maternal scaffolding, it was decided to use a 4-point scale similar to the 4-point children's SBOS to make the scoring process more coherent. The observation criteria were adapted accordingly. This revised coding scheme is described in detail in the Main study section. The changes made in the maternal observation system from the pilot to the main study are summarised in the table below. 
Table 3.1

Changes in the Maternal Observation System after the Pilot Study

Pilot Study
Main Study
A. Cognitive support

- Metacognitive information

- Manner of instruction

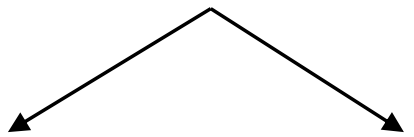

Task difficulty regulation Review

B. Emotional support

- Encouragement

- Rejection

C. Transfer of responsibility

- Mothers overcontrolling

- Mothers' encouragement of active involvement
A. Cognitive support

B. Metacognitive support

- Planning

- Use of strategies and techniques

- Monitoring progress and self-evaluation of the cognitive outcome

C. Emotional and Motivational support

- Positive emotional and motivational support

- Negative criticism-Rejection

D. Autonomy support

- Overcontrolling

- Boosting autonomy and active involvement

\subsection{Main Study}

\subsubsection{Participants}

The participants of this study were thirty-five mother-child dyads who were recruited from state kindergartens. The children's age range was from 54 to 72 months old (mean age = 66 months). Boys comprised the $65.7 \%(N=23)$ of the sample and girls the remaining $34.3 \%$ $(N=12)$. All children attended kindergarten or pre-kindergarten class. Most of the children, that is, $80 \%(N=28)$ attended kindergarten classes, and $20 \%(N=7)$ of the children were in prekindergarten classes.

Maternal age ranged from 30-49 years old. Most of the mothers were 30-39 years old, that is, $82.9 \%$ and the rest $17.1 \%$ were between $40-49$ years old. The mother-child pairs came from two towns in central Greece (Volos and Larissa). All participant mothers were married. 
Mothers formed three groups according to their educational background: Mothers who formed the high educational background group held a postgraduate degree, that is, seven mothers (20\%). The average educational background group had a bachelor's degree and was comprised of 15 mothers (42.85\%). Finally, the low educational background group had a secondary education degree and consisted of 13 mothers (37.14\%).

\subsubsection{Recruitment process}

This study focuses on preschool children recruited from state kindergartens. First, the researcher communicated with several schools in two cities providing information to the school principal and the teachers. The staff was thoroughly informed about the aims of the study and the requirements of the participant children. Then, information flyers were given to the teachers to distribute them to the potential participant mothers considered as suitable candidates (fulfilling the requirements). Children had to meet the following criteria:

1. They had to attend kindergarten or pre-kindergarten classes

2. Children's age at the first phase had to be between 4.5 to 6 years old

3. No children with a diagnosis of developmental, neurological or language difficulties could be included in the research

4. All children and mothers had to speak Greek fluently

After the teacher had informed the mothers and after the distribution of the flyers, mothers expressed their interest to the teacher and signed informed consent, giving their telephone number to be contacted by the researcher. Subsequently, the researcher communicated with the mothers to provide more information about the procedure and the aims of the study and to confirm that they were interested in participating in the study. After mothers have agreed to be involved in the study, an appointment was set either at the school setting or their homes, wherever the mother felt more comfortable. The same procedure was followed in the second phase. The mothers were already informed by the teacher and their interaction with the researcher that almost in five-six months the researcher would reach them again asking to participate in the last phase of the study. Thus, the researcher called the mothers five to six months later, describing the second phase and ensuring their involvement. The meeting place was either the home or the school. 


\subsubsection{Research tools}

This study used questionnaires, observation coding schemes and Visual-spatial and Language tasks to answer the research questions. This unit provides details and information regarding the research tools and measures of the study. First, the questionnaires that were completed by the mothers of the study will be presented. Afterwards, the cognitive tasks that were used for the children-alone condition and the mother-child joint problem-solving will be described for both research times. Finally, the section provides a detailed description of the assessment of children's SRL skills, maternal supportive behaviours and maternal contingency.

Questionnaires. All participant mothers were given oral instructions on how to complete the scales. The majority of questions required a degree of agreement or disagreement in a frequency level, using Likert-type scales. A period of approximately 30 minutes was required to complete all the questionnaires.

Demographic questionnaire. To collect individual information regarding the mothers and children, a demographic questionnaire was developed. Mothers were asked to provide the following information: Date of the participant child's birth, school level, mother's and father's age, occupation and education level.

The tool of Parenting Self-Efficacy (TOPSE) (Kendall \& Bloomfield, 2005). TOPSE

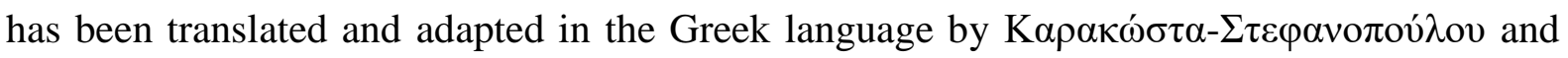

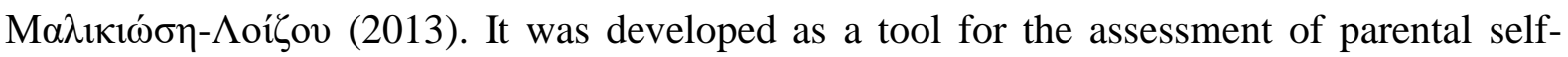
efficacy. It is comprised of 48 items. Kendall and Bloomfield, (2005) hold that the instrument evaluates eight parenting domains, namely Emotion and affection, Play and enjoyment, Empathy and understanding, Control, Discipline and setting boundaries, Pressures, Selfacceptance, and finally, Learning and knowledge. Parents are invited to state their level of agreement for every item on a Likert scale, ranging from 0 (completely disagree) to 10 (completely agree) (e.g., I am consistent in the way I use discipline, It is difficult to cope with other people's expectations of me as a parent, I am able to learn and use new ways of dealing

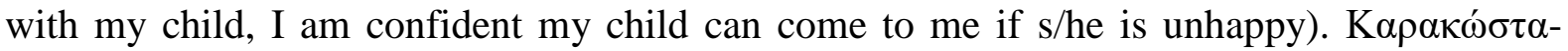

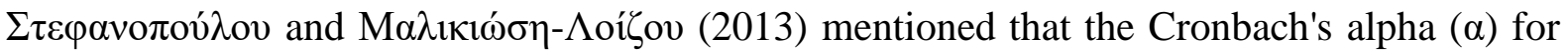
the Greek translated questionnaire was over 0.70 for the most subscales.

The Parenting Styles and Dimensions Questionnaire (PSDQ) (Robinson, Mandleco, Olsen \& Hart, 2001). The Greek adaptation of the maternal PSDQ was carried out by

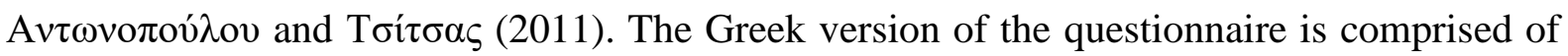


29 items assessing four parenting styles using a Likert scale from 1 (never) to 5 (always). Parents are required to state how often they are engaged in behaviours or situations described in each item [e.g., I explain the consequence of my child's behaviour (authoritative), I slap my child when the child misbehaves (authoritarian), I find it difficult to discipline my child (permissive), I yell or shout when my child misbehaves (strict)]. The internal consistency coefficients for the Greek questionnaires (Cronbach's alpha) were 0.65 for the permissive mother scale, 0.67 for the strict mother scale, 0.83 for the authoritarian mother scale and 0.88

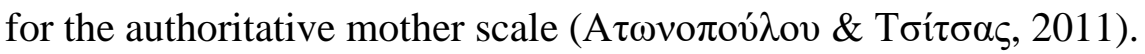

The Children's Behaviour Questionnaire-Very Short Form (CBQ-VSF) (Putnam \& Rothbart, 2006). CBQ-VSF was used for assessing 3-7 years old children's temperament. This tool is comprised of three temperament dimensions, namely Negative Affectivity (e.g., Is afraid of burglars or the "boogie man") Surgency/Extraversion (e.g. Likes rough and rowdy games) and Effortful Control (e.g., When drawing or colouring in a book, shows strong concentration). Parents are asked to answer to 36 items ranging from 1 (completely untrue for the child) to 7 (completely true for the child). The Greek translation and adaptation were

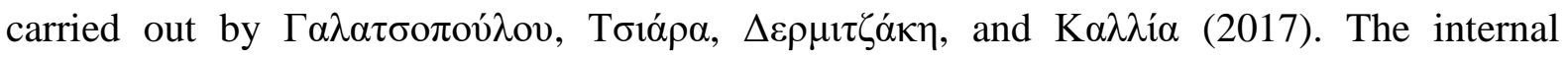
consistency coefficients for the Greek questionnaire (Cronbach's alpha) were 0.74 for Surgency/Extraversion, 0.77 for Negative Affectivity and 0.71 for Effortful Control.

Cognitive tasks. The participant children were asked to perform two different but similar sets of cognitive tasks under two conditions: The first condition referred to the individual problem-solving and the second condition to the joint problem-solving (under the supervision of their mother). The second context was set up only as a necessary condition to record mothers' supportive behaviours. Therefore, the children's cognitive performance was calculated by taking into account only the scores obtained at the first condition, i.e., the individual problem-solving. At the beginning of the examination, just before the individual problem-solving, the child and the researcher practised four examples in the Visual-spatial tasks and one example in the Language task to make sure that the child comprehends what s/he was asked to do.

Time 1 - child alone - Visual-spatial tasks. 30 cubes were given to each child with three different colour sides. The examiner asked the child to reproduce four given patterns of cubes assemblies as they were depicted in cards-models. The tasks required Visual-spatial, arithmetic and analytic abilities. The four different cards presented to the child had increasing difficulty levels (e.g., the last card presented a more difficulty model), from simpler black and white models to more complicated coloured models. Children's performance was calculated 
by the number of the correct fittings (also considering the colour of the cubes). For example, in the first task, the child had to assemble eight cubes to replicate the model depicted in the card-model. For this task, the cubes were not coloured. The child was given a point for each correct placement of the cube (e.g., If the child had placed in the correct place seven cubes the score would be 7 out of 8). The highest score for all the four tasks was 51 .

The following instructions were given for this kind of tasks:

"Do you see these cubes? They are all the same. Each cube has two red sides, two yellow and two blue. This task aims to place the cubes exactly as shown in the picture to form the construction depicted. Now, let's try to assemble these cubes as the figure shows (example). Did you see? This construction we just made is exactly the same as the one shown on this card. Now, try to do the same thing with this figure (example). Now, try to assemble these cubes on your own so that they look exactly as shown in this picture ... Please, do the same with this card".

\section{Picture 3.1}

Examples of Visual-spatial tasks (Time 1)
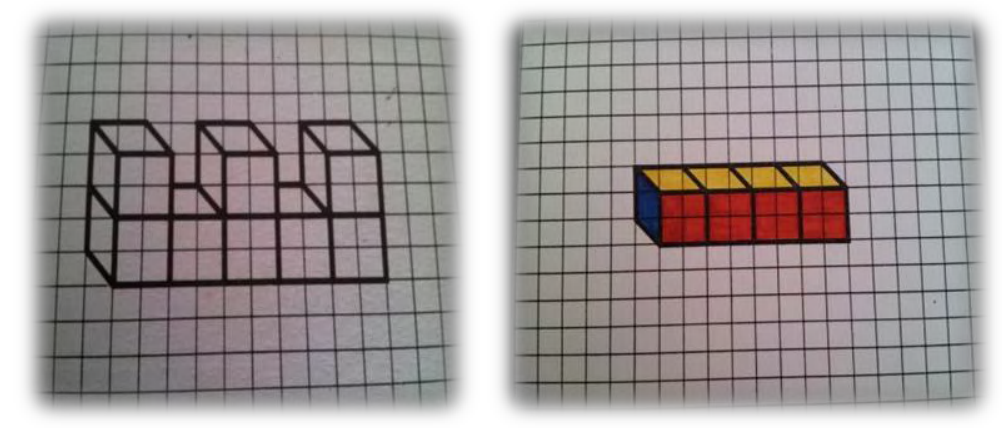

Time 1 - mother-child - Visual-spatial tasks. Four cube tasks were administered to each mother-child pair. The tasks presented gradually more difficult models. If the previous four tasks were too easy for the child to solve, one extra more difficult task was given to the mother-child pair in order to elicit the maternal supportive behaviours. The examiner informed the mother that she had to support her child to come to a solution, when she felt this was necessary, provided with the following instructions:

"There are four tasks to be solved. Some of them might be difficult for children of this age. We do not expect the child to complete all the activities, but we are more interested in the ways your child uses to come to a solution. Please, give instructions to your child or any other help you consider necessary for your child to assemble the cubes as shown in the picture". 
Time 1 - child alone - Language tasks. The Language tasks included one Story Development task. First, the child was given six cards depicting heroes (three cards), objects ( 1 card), and places (two cards). The researcher started developing a story using three of the cards and, next, the child was asked to continue and finish the story using the rest of the cards. Children's performance in the Language task was calculated by three different scores or criteria: Logical thinking and coherence of meanings, Vocabulary, and Structure of the story. Children's performance was rated on a 4-point scale ranging from 1 (low) to 4 (high). The highest score for the Story Development was 12. Detailed information about the scoring criteria is given below in table 3.2.

The following instructions were given for this kind of tasks:

(Story Cards on the table: boy, princess, wolf, mirror, river, shaft)

For the Story Development task: «Do you see these pictures? We are going to develop a fairy tale. We need to be careful so that we use all the cards. I am going to start the story, and then you will continue. Do you understand what you need to do"?

"Now, listen to how the story begins: Once upon a time, there was a poor boy who had no one in his life. He desired to learn how to write and read and be able to go to school. He placed a few things in a bag and left to follow his dream. As he walked for many days and nights, he met a princess. The princess asked the boy why he was barefoot and lonely. The boy explained everything he wanted to do and the princess promised to help him if he could find her golden mirror that someone had stolen. Now, try to continue and finish the story. Please, do not forget: the completed story need to take into account all the six pictures»».

Picture 3.2

The Language Task of the Main Study (Time 1)

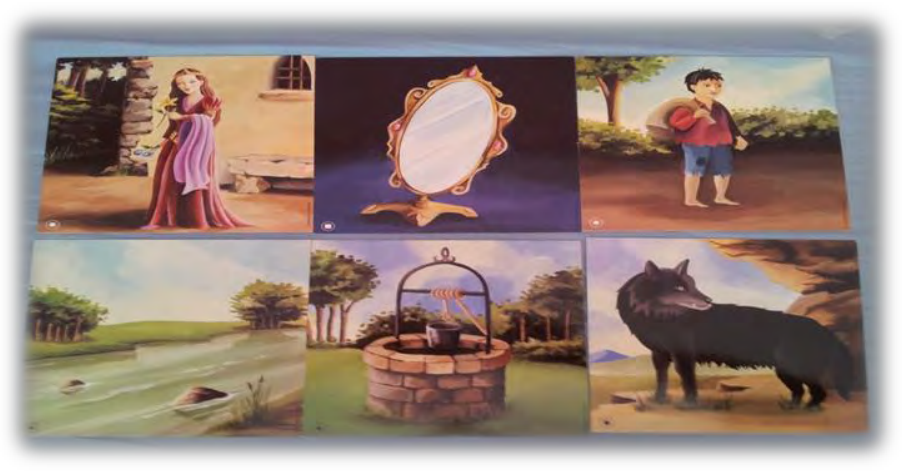


Table 3.2

Scoring Criteria for the Performance in the Language Tasks

\begin{tabular}{|c|c|c|}
\hline Criteria & Definitions & Scoring \\
\hline $\begin{array}{c}\text { Logical thinking } \\
\text { and coherence of } \\
\text { meanings }\end{array}$ & $\begin{array}{l}\text { It includes: } \\
\text { The meaningful continuity, the } \\
\text { rationality of the meanings during } \\
\text { the production of the story, the } \\
\text { cohesive and logical sequence of the } \\
\text { meanings, the connection of the story } \\
\text { with the given pictures and the } \\
\text { child's reference to all of the given } \\
\text { pictures }\end{array}$ & $\begin{array}{l}\text { 1=There is a major lack of coherence in the story. The child describes the pictures } \\
\text { separately one by one, indicating only what he sees, without any attempt to } \\
\text { meaningfully connect them. } \\
\text { 2=The child makes few right connections using the pictures, but the narrative has } \\
\text { many ambiguities. The logic of the story, the coherence of meanings is below } \\
\text { average. During the narrative, there are few referrals to the pictures, and the central } \\
\text { information is missing. } \\
\text { 3=The logical and meaning continuity is good with few ambiguities. The child may } \\
\text { lose some trivial points that generate some confusion or questions. Some interior } \\
\text { information from the pictures is not taken into consideration during the narrative. } \\
\text { 4= The child produces a story with a clear and logical continuity among the } \\
\text { meanings, there is no vagueness in the story or ambiguities (this means that the } \\
\text { child has taken into account logical rules that link the pictures together and places } \\
\text { the cards in a reasonable order). The child develops a story that includes the central } \\
\text { information of the pictures and refers to logical conclusions, telling the story as a } \\
\text { result of a sequence of events. S/he links the six pictures appropriately. }\end{array}$ \\
\hline Vocabulary & $\begin{array}{l}\text { The child's verbal fluency, the } \\
\text { description of the picture with details } \\
\text { while referring to all the significant } \\
\text { parts, heroes, facts, etc. }\end{array}$ & $\begin{array}{l}\text { 1=The vocabulary of the child is generally poor for his/her age. The child uses the } \\
\text { same phrases and words or a minimal range of words. The child has difficulties in } \\
\text { expressing himself/herself, and his/her narrative is restricted to a particular central } \\
\text { point of the story, a single image or hero. } \\
\text { 2=The child uses short sentences that include the basic vocabulary of the pictures } \\
\text { within a limited context. The child may find it hard to express the right words and } \\
\text { often asks for help to recognise and describe the picture's information. }\end{array}$ \\
\hline
\end{tabular}




\begin{tabular}{|c|c|c|}
\hline & & $\begin{array}{l}3=\text { The child describes the pictures with verbal fluency and describes the most of } \\
\text { the important information of the pictures. Sometimes he/she produces short } \\
\text { sentences, but he/she includes complex or primary and secondary information. } \\
4=\text { The child has significant verbal fluency. He/she describes the images in detail } \\
\text { and lists all their important and secondary elements. The child reports information } \\
\text { about the characters, the scenes, their intentions, the initial and final events, their } \\
\text { reactions, etc. }\end{array}$ \\
\hline $\begin{array}{c}\text { Structure of the } \\
\text { story }\end{array}$ & $\begin{array}{l}\text { It examines if the story has distinct } \\
\text { structural parts (e.g. a beginning, a } \\
\text { middle part and an ending) and if the } \\
\text { narration has a closure and if this } \\
\text { ending is connected with the story }\end{array}$ & $\begin{array}{l}\text { 1=The child fails to form distinct parts of the story and is referred randomly to the } \\
\text { picture he/she sees. There is no structure in the story or distinct end or conceptual } \\
\text { "closure". } \\
\text { 2=The narration has one or two parts, but the child often finds difficult to structure } \\
\text { these units. The child opens or closes these parts quickly, and their basic } \\
\text { information is hard to discern. The narration seems to shape a closure, but this } \\
\text { ending is weak to its extent and coherence. } \\
\text { 3=The child understands the important events and connects them in a story where } \\
\text { there are distinct parts. However, sometimes some of these parts are not completed. } \\
\text { The narration has a closure, but it is not completed or equivalent to the previous } \\
\text { parts considering its extent and coherence. } \\
4=\text { The child's story has a clear structure (usually, beginning-middle-end), it } \\
\text { includes the description of all the pictures of the story taking into account the } \\
\text { presented order. The story has a reasonable end, a meaningful "closure". }\end{array}$ \\
\hline
\end{tabular}


Time 1 - mother-child - Language tasks. The examiner informed the mothers that they should develop a story based on the given pictures and, next, to support their child, when necessary, to complete and finish the story. Each pair had to develop one story.

The instructions given to the mothers for the Story Development task were the following:

(Story cards on the table: "Hare, frog, witch, cave, castle, ring")

"We want you to support your child tell a story using all these pictures. You can start by narrating a story while showing the pictures you used to your child. Then, ask your child to continue the story as he/she thinks and finish it. A good idea for the beginning of the story is to start with a hero who lives in a certain place, in a particular situation (for example, he/she is curious, bored, finds no job ...) and wishes to acquire something (e.g., an item). In his/her endeavour to obtain the object, various adventures could happen, or the hero might meet other characters, possible obstacles (at this point you should ask the child to continue the story)".

Time 2 - child alone - Visual-spatial tasks. The block design subtest from WISC-III was used. Each child was given nine cubes and s/he was asked to reproduce four given patterns of cubes assemblies as they were depicted in card-models. The tasks required Visualspatial, arithmetic and analytic abilities. The cubes had two white sides, two red sides and two sides that were half-white and half-red. The four different cards presented gradually more difficult models to be constructed by the child. Two of the cards were exactly as depicted in the WISC-III, and two of the cards were constructed by the researcher. Children's scores were calculated by the number of the correct fittings (also considering the colour of the cubes). The highest score for all the four tasks was 21 .

The following instructions were given for this kind of task:

"Here are nine cubes. They are all the same. Each cube has two red sides, two white sides and two sides that are half-white and half-red. Now, please watch me. I am going to assemble these cubes to form this figure (example). Did you see? This design, I just made, is exactly the same as the one depicted in the picture. Please, try to do the same thing (example). Now, try to assemble these cubes on your own so that they look exactly as this picture shows ... Please, do the same with this card". 


\section{Picture 3.3}

Examples of the Visual-spatial tasks (Time 2)
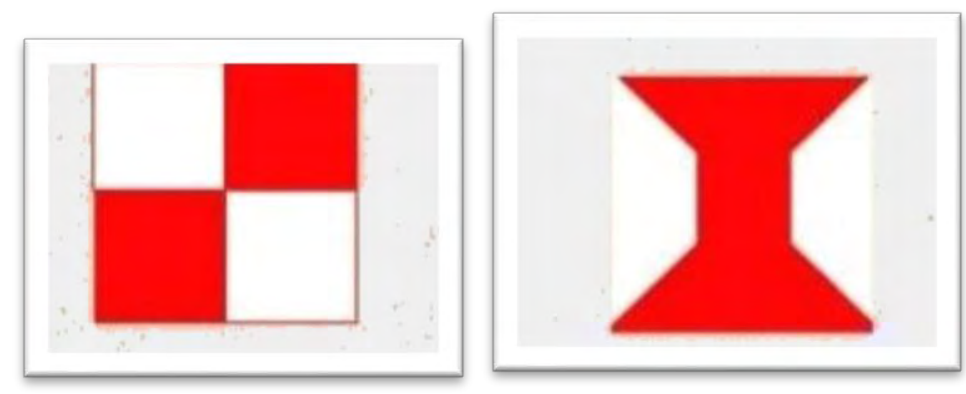

Time 2 - mother-child - Visual-spatial tasks. Each mother-child pair was given five cube tasks of increasing difficulty, taken from WISC-III. As in Time 1, the researcher informed the mother that she could support her child to come to a solution when she felt this was necessary. The following instructions were given to the mother: "We have chosen five tasks, some of them may be difficult for children of this age. We are interested in the ways your child tries to solve these tasks. Please, give instructions to your child or any other help you consider necessary to support your child to assemble the cubes as shown in the picture".

Time 2 - child alone - Language task. The Language task in Time 2 was the same as in Time 1; however, different pictures were given to the child. The child was given again six cards depicting heroes (three cards), objects (1 card), and places (two cards). The researcher began developing an oral story using three of the cards and, next, she asked the child to continue and finish the story using the rest of the cards. The performance criteria were the same as in Time 1, including Logical thinking and coherence of meanings, Vocabulary, and Structure of the story. Children's performance was rated on a 4-point scale ranging from 1 (low) to 4 (high). The highest score for the Story Development was 12.

For the Story Development task, the child was given the following instructions:

(Story Cards on the table: wizard, dwarf, squirrel, a cabin with straws, tree, boots)

"Do you see these pictures? We are going to try to make a fairy tale. We need to be careful so that we use all the cards. I am going to start the story, and then you will continue. Do you understand what to do? Now, listen to how the story begins: Once upon a time, there was a wizard. Wherever he went, he wanted to help those who needed him. He was meeting animals and people asking them "Tell me what do you need to be happy"? One day he arrived in a village where a dwarf lived. The wizard asked him: "Tell me dwarf, what do you need to be happy?" The dwarf told him he would like to help all the forest animals to build their nests. His wish was to be able to run faster so that he could help all the animals in the forest. 
Then, the wizard told him that there was a pair of magic boots hidden in the woods. If the dwarf could find the boots, he could run so fast as lightning".

The researcher continued: "So far, I've used three of the six cards, and I've made the story you heard. You should continue the story using the rest of the cards as you like ...»».

Time 2 - mother-child - Language tasks. As in Time 1, mothers were asked to start developing a story based on the given pictures and, next, to help their child, to complete and finish the story. Again, each pair had to develop one story. The instructions given to mothers were the same as in the first phase, although the pictures were different including a forest, a princess, a prince, a sword, a dragon and a bridge.

Children's SRL skills. To assess children's actual strategic behaviours during problem-solving, that are indicative of SRL, the SBOS (Dermitzaki et al., 2009) was used. Six children's strategic behaviours were assessed during solving the cognitive tasks: 1 . Cognitive strategies, such as Choosing between main and trivial information, Analysing and Combining activities, etc., 2. Planning of the solution process, 3. Self-monitoring during problem-solving, 4. Concentration/interest, 5. Persistence in front of difficulties, and 6. Working autonomously. Examples of the scoring and the description of strategic behaviours are given below in table 3.3. 
Table 3.3

Examples of the Description and Scoring of Children's SRL Skills (Dermitzaki et al., 2009)

\begin{tabular}{|c|l|l|l|}
\hline $\begin{array}{c}\text { Children's } \\
\text { SRL skills }\end{array}$ & \multicolumn{1}{|c|}{ Description } & \multicolumn{1}{c|}{ Scoring } \\
\hline $\begin{array}{c}\text { Cognitive } \\
\text { behaviours }\end{array}$ & $\begin{array}{l}\text { It refers to the child's ability to discern the main } \\
\text { and the trivial information of the task. It also } \\
\text { includes the ability to divide and combine different } \\
\text { components to make a whole. Finally, it involves } \\
\text { the ability of the child to use correctly and } \\
\text { efficiently the card model to construct the task. }\end{array}$ & $\begin{array}{l}\text { 4=The child uses efficiently and effectively the card- } \\
\text { model, he/she systematically chooses to deal with the } \\
\text { important elements, and he/she always succeeds in } \\
\text { combining the individual parts of the activity. }\end{array}$ \\
\hline $\begin{array}{c}\text { Metacognitive } \\
\text { skills }\end{array}$ & $\begin{array}{c}\text { Planning of the } \\
\text { solution } \\
\text { process }\end{array}$ & $\begin{array}{l}\text { It refers mainly to mental processes plans before the } \\
\text { beginning of the problem solving as evident during } \\
\text { the child's efforts. It includes the child's efforts to } \\
\text { understand what the problem is about, the aim of } \\
\text { the problem, how to plan the solution process etc. }\end{array}$ & 4=The child uses a clear plan and makes effective use \\
of the time.
\end{tabular}


Scoring. Two independent judges rated each child's problem-solving skills for each one of the cognitive tasks using a 4-point scale from 1 (clear lack of this strategy) to 4 (constant and systematic use of this strategy). Specifically, the final score for each one of the SRL strategies examined was the mean score of the ratings of the two judges for the particular skill. For example, the final score for the Cognitive skills used by the child in the first task of the Visual-spatial domain was the mean score of the scores given by the two judges in this particular task and skill respectively. Considering that the Visual-spatial tasks given to children were four in phase 1, there were four scores assessing children's Cognitive skills, four scores for their Planning skills, four scores for the Self-monitoring skills and so on. The mean scores of these four ratings were calculated to estimate the total Cognitive skills score, the total Planning score, the total Self-monitoring score and so on, for the Visual-spatial tasks. In the Language task, there was only one score for each one of the assessed skills, as there was only one task. In the end, for every child, there were six total scores for each one of the four tasks in the Visual-spatial domain (one for the Cognitive skills, one for the Planning, one for the Self-monitoring, one for the Concentration, one for the Persistence and one for the ability to Work autonomously) and respectively six scores in the Language domain. The whole process is presented in the following example (table 3.4).

Table 3.4

Example of Assessing Children's SRL Skills in the Visual-spatial tasks

\begin{tabular}{|c|c|c|c|c|c|c|c|c|c|}
\hline Visual-spatial & \multicolumn{2}{|c|}{ Card 1} & \multicolumn{2}{|c|}{ Card 2} & \multicolumn{2}{|c|}{ Card 3} & \multicolumn{2}{|c|}{ Card 4} & \multirow{3}{*}{$\begin{array}{c}\text { Total SRL } \\
\text { Skills } \\
\text { and } \\
\text { performance } \\
\end{array}$} \\
\hline \multirow{2}{*}{$\begin{array}{l}\text { Cognitive } \\
\text { skills }\end{array}$} & Judge1 & Judge2 & Judge 1 & Judge2 & Judge1 & Judge2 & Judge1 & Judge2 & \\
\hline & 1234 & 1234 & 1234 & 1234 & 1234 & 1234 & 1234 & 1234 & \\
\hline $\begin{array}{l}\text { Mean of } \\
\text { Cognitive } \\
\text { skills }\end{array}$ & \multicolumn{2}{|c|}{2.5} & \multicolumn{2}{|c|}{3} & \multicolumn{2}{|c|}{2} & \multicolumn{2}{|c|}{2} & 2.375 \\
\hline \multirow{2}{*}{ Planning } & Judge 1 & Judge2 & Judge 1 & Judge2 & Judge 1 & Judge2 & Judge1 & Judge2 & \\
\hline & 1234 & 1234 & 1234 & 1234 & 1234 & 1234 & 1234 & 1234 & \\
\hline
\end{tabular}

Maternal supportive behaviours. After the pilot study, a revised structured observation scheme was employed to record different maternal supportive behaviours deployed during joint mother-child problem-solving. This tool originated and was adapted from the Neitzel and Stright's observation system (2003) for parents, as described in the Pilot study section. The adapted observation system records the frequency of specific maternal 
behaviours or practices activated spontaneously during mother-child joint learning and problem-solving. The observation system assesses eight different supportive behaviours that presumably tap the following aspects of supportive behaviour: Cognitive support, Metacognitive support, Emotional-Motivational support, and children's Autonomy support.

The first aspect, Cognitive Support, refers to adult's hints, clues and prompts provided to the child in order to better understand the task and its characteristics, to elaborate the cognitive material, to distinguish the main points from the trivial information, to analyze and combine information, etc. (e.g., the mother tells the child "can you count, how many cubes do we need?").

The second aspect was Metacognitive support which includes the adult's hints, clues and prompts to enable the child to strategically plan the activity towards achieving the cognitive goal, to effectively implement strategies, to self-monitor the problem-solving process and to evaluate the cognitive outcome. Maternal Metacognitive support assesses three specific supportive behaviours: (a) Planning takes place before and during dealing with the task and includes the mother's hints, clues and prompts in order to assist her child to organize the steps needed for the solution process, (e.g., the mother asks the child "First, which part of the model should you make? ...Let's make this part of the construction"); (b) Use of strategies and techniques takes place during the child's involvement with the task and includes the maternal information, hints, clues and prompts for supporting the child to produce and use effectively specific strategies and ideas to manage the task and to improve the solution process, (e.g., "We should begin with the easier stuff first"), and justifications on what, why, when, and how to use a particular strategy, (e.g., the mother tells the child "This construction looks like stairs. The second step has to be placed on the first, so you need a base to build it"); (c) Monitoring progress and self-evaluation of the cognitive outcome take place during the involvement with the task and after its end and it refers to the adult's hints, clues and prompts to support the child to focus on and evaluate the progress made so far in relation to the final goal, to discover his/her mistakes in order to correct them, or when the adult summarises the steps made so far (e.g., the mother tells the child "So far, we've built the first and second step, now which step are we going to make?").

The third aspect was Emotional and Motivational support with two different behaviours assessed: (a) Positive emotional and motivational support: This aspect includes adult behaviours, verbal and nonverbal, indicative of a positive emotional and motivational climate of the interaction. Examples are providing positive reinforcement that motivates the child, recognition of the child's effort, prevalence of positive emotions with a verbal or non- 
verbal manner, such as use of encouraging words, positive comments about the work or the child's ability to solve the task, a positive emotional tone of voice, facial expressions, etc. This maternal aspect also refers to the mother's efforts to attract and retain the child's interest and motivation towards the task, such as focusing on enjoying the process, expressing positive expectations, etc. (e.g., "What a great idea", "You are doing great", "Keep on trying, I know it's hard", "Wow, you are really concentrated on that"); (b) Negative criticism rejection focuses on the prevalence of negative affect between adult-child and overt or hidden disapproval of the child's activity or choices. Verbal and nonverbal behaviours indicating criticism, disapproval, rejection of children's efforts, such as redirection of the child in a negative way, non-verbal signs of disapproval (e.g., gestures, facial expressions, disgust, tone of voice) or other negative or inappropriate reactions to the child were recorded (e.g., "You do not pay attention to what I am telling you", "I knew you wouldn't do that").

The last aspect examined was Children's Autonomy support which is comprised of two different behaviours: (a) Boosting autonomy and active Involvement refers to the extent to which the adult encourages the independent involvement of the child in the problemsolving process, uses prompts, hints, and questions to help the child discover some next step or/and to achieve a personal progress towards the desired solution (e.g., "You can begin the construction on your own and I will help you if you need it", "What do you think you should next?"). (b) Over-controlling which is assessed by the extent to which the adult intervenes to control over the child's efforts to solve the task despite the fact that the child can work independently, when s/he tells the child what to do, directs the child into her own way or opinions for solving the task and, generally, when the adult imposes his/her will or own plan or strategies towards a solution (e.g., "Now, put this cube next to the other, I want to check what you are doing", "Don't ask why this is the way it has to be done"). A detailed description of the scoring criteria is presented below in table 3.5. 
Table 3.5

Examples of the Description and Scoring Criteria for the Maternal Supportive Behaviours

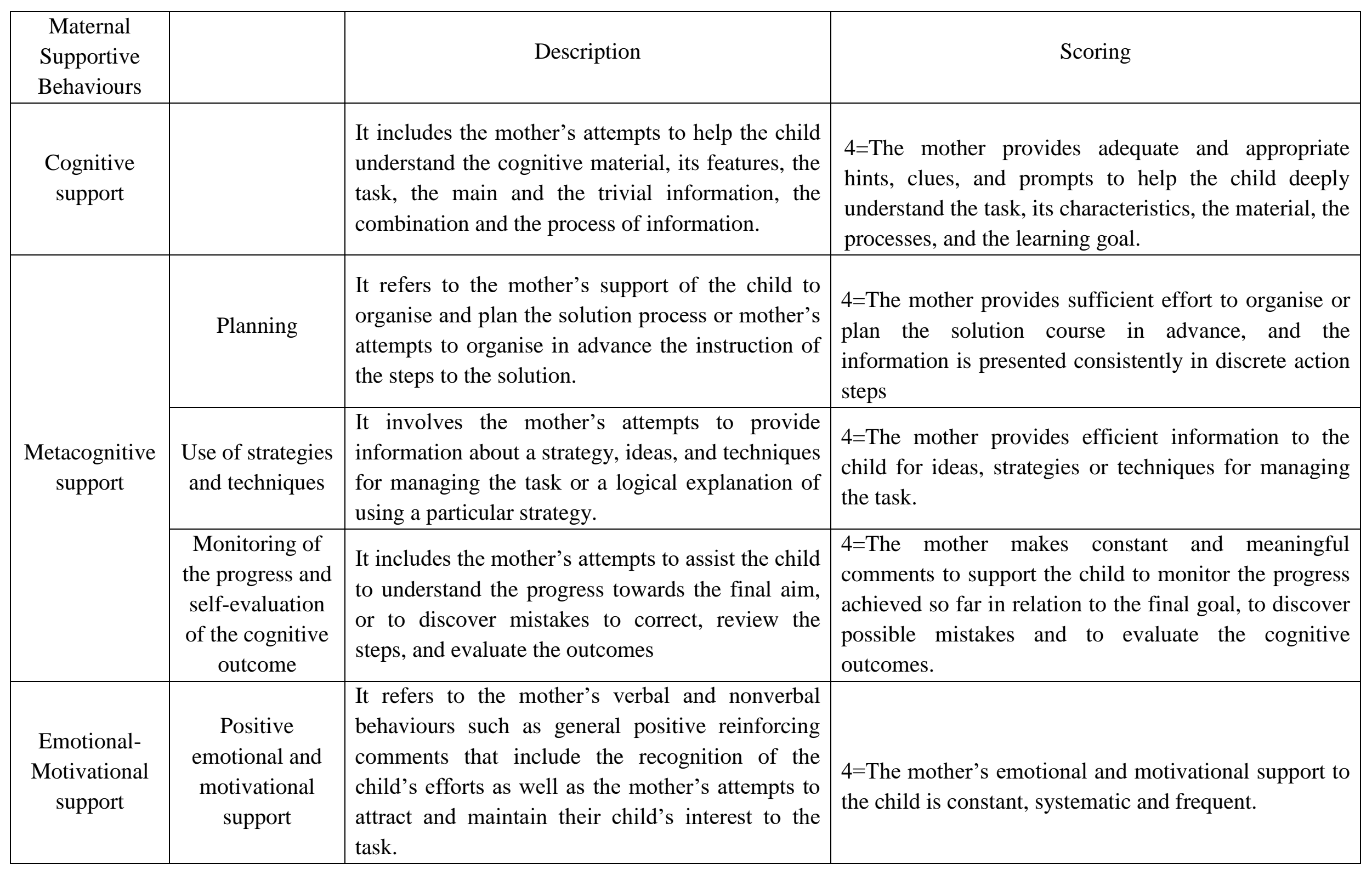




\begin{tabular}{|c|c|c|c|c|}
\hline \multirow{1}{*}{$\begin{array}{c}\text { Autonomy } \\
\text { support }\end{array}$} & $\begin{array}{c}\text { Negative } \\
\text { criticism- } \\
\text { rejection } \\
\text { autonomy and } \\
\text { active }\end{array}$ & $\begin{array}{l}\text { It refers to mother's wording or signs of criticism, } \\
\text { disapproval or rejection of the child's efforts. It } \\
\text { also includes nonverbal responses (e.g., facial, the } \\
\text { tone of voice) or other inappropriate reactions } \\
\text { towards the child. }\end{array}$ & $\begin{array}{l}\text { It refers to the mother's encouragement of the } \\
\text { child's independent cognitive involvement in the } \\
\text { solution process. }\end{array}$ & $\begin{array}{l}\text { 4=The mother systematically and consistently responds } \\
\text { 4hegatively to the child and makes negative comments. } \\
\text { the child's autonomy and active involvement through } \\
\text { questions, prompts, hints and clues. }\end{array}$ \\
\hline Overcontrolling & $\begin{array}{l}\text { It refers to the mother's tendency to interfere and } \\
\text { to control the behaviours of her child and the } \\
\text { solution process instead of helping the child to be } \\
\text { autonomous. }\end{array}$ & $\begin{array}{l}\text { 4=The mother controls the interaction and constantly } \\
\text { interferes with the solution process, despite the fact } \\
\text { that the child can work independently. }\end{array}$ \\
\hline
\end{tabular}


Scoring. The different aspects of maternal supportive behaviours were carefully described to facilitate the observers to discern with adequate precision the behaviours included in each category. Two judges based on these criteria gave their independent ratings for each of the eight maternal behaviours assessed in each joint task on a 4-point scale. First, the two judges studied and then discussed the measures and the scoring criteria. Afterwards, they practised independently in cases from the pilot study and discussed difficulties, doubts or disagreements in scoring. After further agreement and clarification were met, they two judges coded the voice recordings of the main study.

One point was given when the mother did not provide any or very rare support to the child, and four points were given when the mother was giving constant and systematic support to the child. The final score for each maternal behaviour was the mean of the two observers' ratings. For example, maternal Cognitive Support in the first task of the Visualspatial domain was the mean score of the two scores given by the judges. Considering that the Visual-spatial tasks given to the mother-child dyad were four in Time 1, there were four scores assessing maternal Cognitive Support, four scores for maternal Planning, four scores for maternal Negative Criticism and so on. The mean score of these four ratings was calculated to estimate the total maternal Cognitive Support, the total maternal Planning, the total maternal Negative Criticism and so on, for the Visual-spatial tasks. In the Language task, as there was only one task, there was accordingly one score for each one of the assessed maternal supportive behaviours. Therefore, there were eight scores for the supportive behaviours for each one of the Visual-spatial tasks and eight scores for the Language tasks. The process of scoring is presented in the following example table (table 3.6). 
Table 3.6

Example of Scoring Maternal Supportive Behaviours in the Visual-spatial Tasks

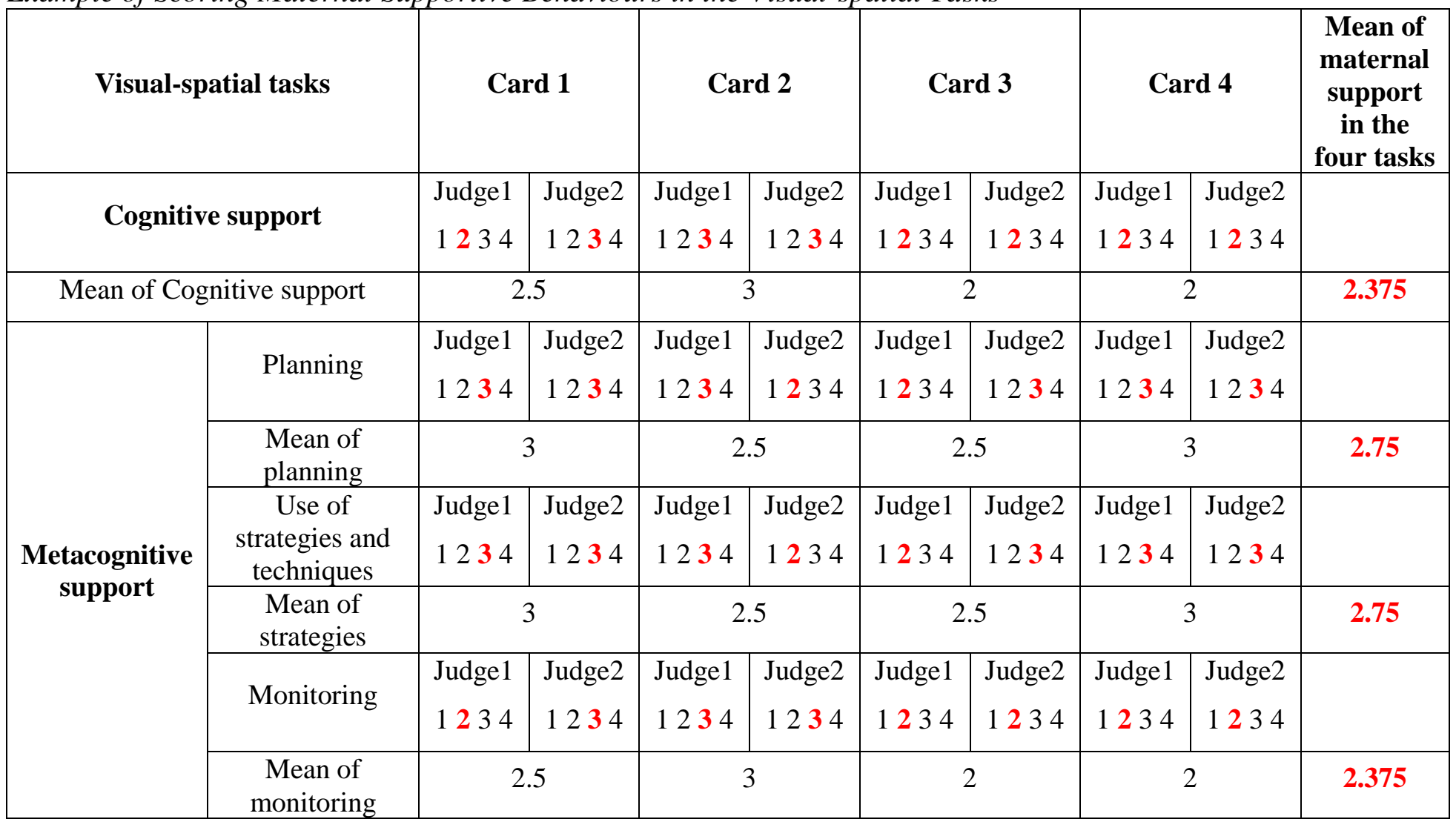

Maternal contingency. The extent to which the maternal supportive behaviours are related to children's SRL skills and performance depends on the contingency with which are provided. In the present context, the mothers not only have to provide cognitive, metacognitive and emotional support but they also have to adjust the level of support to their child's stage of understanding or mastery of the task, to provide effective assistance.

Maternal contingency was assessed in this thesis after each task of a particular cognitive domain. A general impression score was given ranging from 0 (the mother does not respond to the children's needs) to 3 (the mother provides contingent support). This scoring system was used to estimate the extent to which the mother provided support to the child according to the level of his/her understanding and skills. Specifically, maternal contingency was defined as the level to which the mother identifies and responds effectively to the real cognitive and emotional needs of the child to solve the task. In this respect, we were mostly interested in capturing if the mother's support is attuned to the level of the child's understanding and if it is coordinated with the child's possible changes in the degree of his/her understanding. The following table (table 3.7) describes the scoring criteria for maternal contingency. 
Table 3.7

Maternal Contingency: Mother's Responsiveness to the Child's Understanding and Cognitive Needs

\begin{tabular}{|c|c|}
\hline Maternal contingency & Scoring \\
\hline $\begin{array}{l}\text { It refers to the degree to which the mother perceives and } \\
\text { responds successfully to the child's real cognitive needs } \\
\text { in order for the child to solve the task. It refers mainly to } \\
\text { the degree to which the mother's responses are attuned } \\
\text { with the level of the child's understanding and to the } \\
\text { extent to which the mother adapts her behaviour or } \\
\text { coordinates her behaviour to possible fluctuations in her } \\
\text { child's level of understanding, based on the signs of } \\
\text { difficulty experienced by the child. }\end{array}$ & $\begin{array}{l}\mathbf{0}=\text { the mother does not respond or offer support during the solution process, } \\
\text { either because she is not interested, or she can not address the level of her } \\
\text { child's understanding. } \\
\mathbf{1}=\text { the mother tries to participate and provide support, but she cannot provide } \\
\text { appropriate responses, or she cannot adapt appropriately her } \\
\text { instructions/behaviours to the level of her child's understanding or difficulty. } \\
\text { The mother either (a) demands more from the child and continues to provide } \\
\text { instructions that the child cannot understand or (b) insists on helping the child } \\
\text { when the child does not need her assistance or (c) provides less support than the } \\
\text { child needs. } \\
\mathbf{2}=\text { the mother participates and provides appropriate responses according to her } \\
\text { child's level of understanding and level of difficulty. Sometimes, she is not able } \\
\text { to attune her support to the child's needs. She may demand more of her child or } \\
\text { over-facilitate her child's efforts or provide less assistance than the child needs. } \\
\mathbf{3}=\text { the mother offers support when the child needs help and provides } \\
\text { appropriate responses according to her child's level of understanding or level of } \\
\text { difficulty. When the child succeeds, the mother does not intervene, but she } \\
\text { supports (e.g., emotionally or motivationally) her child with an appropriate way } \\
\text { to move on to the next difficulty level. }\end{array}$ \\
\hline
\end{tabular}


Scoring. Scores for the maternal contingency were attributed for every task of both cognitive domains in both research phases by one judge. For example, in the Visual-spatial tasks of the first phase mothers were given four such scores, one for every task, and one score for the Language task. The overall level of contingency was assessed by estimating the mean of the four scores in the Visual-spatial tasks (table 3.11). As there was one task in the Language domain, the contingency score given to this task was also the overall level of contingency.

\subsection{Ethical Considerations}

This study had to reflect on some ethical issues. The first concern of the current study was to secure the safety and rights of the participants. The design of the study and the fact that included children led to a careful examination of the ethical issues that might have been aroused.

For that reason, the first concern was to provide a full description and information about the study procedure to the mothers and the teachers. However, there was an issue on how much details the mothers should know regarding the observation of their behaviour during the interaction with their child. If mothers were informed about the particular aim of the study, then they would likely change the way they usually interact with their child, resulting in low ecological validity. Therefore, the mothers were informed that the researcher was interested in the way children learn with the assistance of their mothers. After that, informed consent from the mother was a necessary step to include the children and the mother to the study in both research times.

A second challenge concerned the use of the voice recordings and the meetings that took place at the mothers' homes or in school settings. Initially, the researcher aimed to use video recordings to better capture the verbal and nonverbal children's and mothers' behaviours. However, the mothers of the pilot study expressed concerns regarding this approach. Therefore, it was decided to use a voice recorder instead, as the mothers felt more comfortable with this method.

The inform consent required from the mothers to state their preference concerning the meeting place in order to solve issues of privacy. The anonymity of the participants was secured as the records were carefully secured and encrypted and no information about the name of participants was used. The mothers were informed that their personal details would not be shared and that the data would be used for research purposes only. In general, the 
procedure was planned to be friendly and familiar to children. The mothers and the children did not express feelings of tiredness or nervousness.

\subsection{Validity and Reliability of the Study}

This section discusses the steps that were made to ensure the quality and status of the study from the view of validity and reliability as well as the attempts to satisfy specific research consistency criteria.

Content validity of the measures. The relevance and representativeness of items were checked during the pilot study, conducted before the main study, involving people with parallel characteristics with those of the main study's participants.

Construct validity. This study relied on two main concepts, that of maternal supportive behaviours during joint problem-solving, and that of children's SRL skills. Specific criteria and definitions were given for both concepts, and both verbal and nonverbal responses were taken into consideration. Neitzel and Stright's (2003) and Dermitzaki et al. (2009) observation systems were used to guide the assessment of specific behaviours.

External validity. The greater limitation of this study concerns its external validity by means of generalisation. First of all, the selection of the sample was not random, and it included a relatively small number of participants. Secondly, mothers came from two cities in Central Greece and were mostly highly educated mothers. This issue constraints the generalisation of the results to other groups and demands a cautious interpretation of the study's outcomes.

Reliability. A specific procedure was followed by the researcher as described above to ensure the reliability of the procedure and data. To increase the reliability of the scoring system used to assess children's and mothers' behaviours, a second judge participated in the assessment of the maternal supportive behaviours and children's SRL skills at Time 1 to estimate the level of agreement. To assure the reliability of the questionnaires used in this study, the internal consistency of the questionnaires was taken into account. All the questionnaires (except for the CBQ) had been translated and adapted in the Greek language, and the Cronbach's alpha coefficient was given. The CBQ was translated and adapted in the

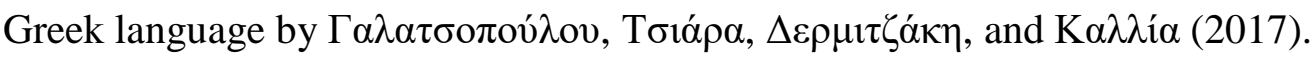




\subsection{Statistical Analyses}

The following chapter describes the statistical analyses, the tasks and the measures used to address the main research questions. In total, 70 audio recordings (35 from time 1 and 35 from time 2) and 35 questionnaires were coded. Descriptive analysis was used to describe or summarise the information gathered and was the starting point for the preliminary depiction of the data (e.g., children's gender, maternal education).

To examine which type of support was activated by mothers more frequently and which SRL skills were employed by pre-school children more frequently during problemsolving, within-subject ANOVAs and the Friedman Test were chosen, depending on the normality of the data.

To explore possible relations between children's and mother's characteristics, demographic variables, maternal supportive and contingent behaviours and children's SRL skills and performance, Spearman's correlation coefficient was used (e.g., for children's age, children's temperament).

To investigate whether and which of the maternal supportive and contingent behaviours were important (cross-sectionally and longitudinally) for young children's SRL and performance partial correlations (controlling for children's age) and multiple regression analyses were run. Mediation analyses were also performed in some cases, to test for indirect effects.

To explore how the maternal supportive and contingent behaviours and children's SRL skills developed from Time 1 to Time 2, Paired t-tests (for the parametric variables) and the Wilcoxon signed rank test (for non-parametric variables) were run. The same statistical tests were employed to investigate whether the participant mothers and children differentiate their behaviours according to the type of task at hand. 


\section{Chapter 4 Results}

This chapter presents the statistical analyses of the data of the two research times of the study. At the beginning of the chapter, the procedure of the data preparation and the preliminary analyses are included such as the Interrater Reliability, the Descriptive Statistics and information about the Normal Distribution of the study's variables. The next section presents the results of the analyses aiming at exploring differences in the types of maternal support and children's SRL skills. Subsequently, demographic, maternal and children characteristics are being studied in order to investigate their relations with the main variables of this study. Over the next section, the possible links between maternal supportive and contingent behaviours and children's SRL skills and performance, at Time 1, at Time 2 and lastly, from Time 1 to Time 2 are presented. The two last sections investigate differences between the maternal contingent and supportive behaviours at Time 1 and at Time 2 and respectively, between children's SRL skills at Time 1 and at Time 2. In addition, the effects of the type of task on the above variables are examined.

\subsection{Preliminary Analyses of the Data}

\subsubsection{Interrater reliability}

To assess the reliability of the scoring systems, the agreement between a first judge (the researcher) and an external judge was computed for the measures at Time 1. Before engaging in independent scoring, the two judges jointly scored and discussed trial cases both from the pilot study and from the main study using the scoring criteria. After reaching agreement on these cases, both judges separately scored the rest of the cases. Each judge scored 35 mother-child interactions for assessing maternal supportive behaviours in the Visual-spatial tasks and 35 mother-child interactions for the Language tasks. For the childalone tasks, only the Language tasks were scored by the second judge, as the voice recordings from the Visual-spatial tasks rarely included verbal behaviours.

Therefore, the intraclass correlation coefficients of the ratings of the two judges for each of the eight maternal behaviours in the Visual-spatial and Language tasks, for each of the six children's SRL skills, and for performance in the Language task were calculated. Table 4.1 presents the inter-rater reliability for the maternal supportive behaviours and table 4.2 the inter-rater reliability for children's SRL skills and performance in the Language task. For the mother-child Visual-spatial tasks, the mean intra-class correlation coefficient for the 
eight maternal behaviours ranged from .89 to 1.0. For the mother-child Language task, the mean intra-class correlation coefficient for the eight maternal behaviours ranged from .96 to 1.0. For the child-alone Language task, the mean intra-class correlation coefficient for the six SRL skills and performance ranged from .94 to .98 . The results show a very high level of agreement between the two judges.

Table 4.1

Inter-Rater Reliability for Maternal Supportive Behaviours - Time 1

\begin{tabular}{|c|c|c|c|}
\hline & & \multicolumn{2}{|c|}{ Intraclass correlation coefficients } \\
\hline & & Visual-spatial tasks & Language tasks \\
\hline \multicolumn{4}{|c|}{ Maternal supportive behaviours } \\
\hline $\begin{array}{l}\text { Cognitive } \\
\text { support }\end{array}$ & Cognitive support & .95 & .97 \\
\hline \multirow{3}{*}{$\begin{array}{l}\text { Metacognitive } \\
\text { support }\end{array}$} & Planning & .99 & .98 \\
\hline & $\begin{array}{c}\text { Use of strategies and } \\
\text { techniques }\end{array}$ & 1.0 & .96 \\
\hline & $\begin{array}{l}\text { Monitoring of the } \\
\text { progress and self- } \\
\text { evaluation of the } \\
\text { cognitive outcome }\end{array}$ & .98 & 1.0 \\
\hline \multirow{2}{*}{$\begin{array}{l}\text { Emotional- } \\
\text { motivational } \\
\text { support }\end{array}$} & $\begin{array}{l}\text { Positive emotional } \\
\text { and motivational } \\
\text { support }\end{array}$ & .99 & .99 \\
\hline & $\begin{array}{l}\text { Negative criticism- } \\
\text { rejection }\end{array}$ & .99 & .97 \\
\hline \multirow[t]{2}{*}{$\begin{array}{l}\text { Autonomy } \\
\text { support }\end{array}$} & $\begin{array}{l}\text { Boosting autonomy } \\
\text { and active } \\
\text { involvement }\end{array}$ & .99 & 1.0 \\
\hline & Overcontrolling & .89 & 1.0 \\
\hline
\end{tabular}


Table 4.2

Inter-Rater Reliability for Children's SRL Skills and Performance in the Language Tasks - Time 1

Intraclass correlation

coefficients

\begin{tabular}{ccc}
\hline Children's SRL behaviours and performance & .98 \\
Cognitive skills & Cognitive skills & .94 \\
$\begin{array}{c}\text { Metacognitive } \\
\text { skills }\end{array}$ & Planning of the solution process & .96 \\
$\begin{array}{c}\text { Emotional- } \\
\text { motivational } \\
\text { skills }\end{array}$ & Self-monitoring during problem-solving & .95 \\
Autonomy skills & Persistence in the face of difficulties & .94 \\
& Working autonomy & .94 \\
Performance & Logical thinking and coherence of \\
& meanings \\
Vocabulary & .98 \\
& Structure of the Story & .98 \\
\hline
\end{tabular}

\subsubsection{Descriptive statistics}

Table 4.3 illustrates the descriptive statistics (means and standard deviations) for the eight maternal supportive behaviours and maternal Contingency and the six children's SRL skills and performance in the Visual-spatial and Language tasks at both research times. The highest score for maternal Contingency was three points, and the lowest score was zero points while the highest score for the maternal supportive behaviours was four points and the lowest score was one point. The scoring system was the same for both research times and both types of tasks. Children's highest rating for the SRL skills was four points, and the lowest was one point for both research times and type of tasks. Children's highest performance score was 51 points for the Visual-spatial tasks at Time 1 and 21 points at Time 2. Children's highest performance score in the Language tasks was 12 points for both research times. 


\section{Table 4.3}

Descriptive Statistics of the Measures

\begin{tabular}{|c|c|c|c|c|c|}
\hline & \multirow[t]{3}{*}{ Time } & & & & \\
\hline & & \multicolumn{2}{|c|}{ Visual-spatial tasks } & \multicolumn{2}{|c|}{ Language task } \\
\hline & & $M$ & $S D$ & $M$ & $S D$ \\
\hline \multicolumn{6}{|l|}{ Mothers' measures } \\
\hline \multirow{2}{*}{ Maternal contingency } & Time 1 & 2.38 & .58 & 2.00 & .59 \\
\hline & Time 2 & 2.22 & .61 & 1.83 & .78 \\
\hline \multirow{2}{*}{ Cognitive support } & Time 1 & 2.62 & .59 & 2.24 & .87 \\
\hline & Time 2 & 3.03 & .47 & 2.6 & .69 \\
\hline \multirow{2}{*}{ Planning } & Time 1 & 2.38 & .62 & 2.05 & .79 \\
\hline & Time 2 & 2.91 & .58 & 2.48 & .89 \\
\hline \multirow{2}{*}{ Use of strategies and techniques } & Time 1 & 2.46 & .54 & 1.87 & .73 \\
\hline & Time 2 & 3.03 & .49 & 2.51 & .82 \\
\hline \multirow{2}{*}{$\begin{array}{l}\text { Monitoring of the progress and self- } \\
\text { evaluation of the cognitive outcome }\end{array}$} & Time 1 & 2.66 & .65 & 2.17 & .82 \\
\hline & Time 2 & 3.1 & 55 & 2.66 & .76 \\
\hline \multirow{2}{*}{ Positive emotional and motivational support } & Time 1 & 2.37 & .74 & 2.07 & .77 \\
\hline & Time 2 & 2.34 & .82 & 1.68 & .83 \\
\hline \multirow{2}{*}{ Negative criticism-rejection } & Time 1 & 1.7 & .47 & 1.57 & .73 \\
\hline & Time 2 & 1.98 & .78 & 1.86 & .84 \\
\hline \multirow{2}{*}{ Boosting autonomy and active involvement } & Time 1 & 2.67 & .70 & 2.66 & .64 \\
\hline & Time 2 & 3.1 & .83 & 2.77 & .97 \\
\hline \multirow{2}{*}{ Overcontrolling } & Time 1 & 2.41 & .62 & 2.48 & 1.07 \\
\hline & Time 2 & 2.37 & .66 & 2.57 & 1.09 \\
\hline \multicolumn{6}{|l|}{ Children's measures } \\
\hline \multirow{2}{*}{ Cognitive skills } & Time 1 & 2.86 & .66 & 2.01 & .99 \\
\hline & Time 2 & 3.35 & .57 & 2.6 & .81 \\
\hline \multirow{2}{*}{ Planning of the solution process } & Time 1 & 2.73 & .79 & 1.9 & .78 \\
\hline & Time 2 & 3.22 & .66 & 2.46 & .78 \\
\hline \multirow{2}{*}{ Self-monitoring during problem-solving } & Time 1 & 2.74 & .80 & 2.02 & .92 \\
\hline & Time 2 & 3.20 & .59 & 2.34 & .87 \\
\hline \multirow{2}{*}{$\begin{array}{l}\text { Concentration/ } \\
\text { Interest }\end{array}$} & Time 1 & 3.36 & .53 & 2.8 & .76 \\
\hline & Time 2 & 3.42 & .54 & 2.66 & .76 \\
\hline \multirow{2}{*}{ Persistence in the face of difficulties } & Time 1 & 3.35 & .63 & 2.56 & .77 \\
\hline & Time 2 & 3.47 & .50 & 2.43 & .85 \\
\hline \multirow{2}{*}{ Working autonomy } & Time 1 & 3.6 & .49 & 2.72 & .78 \\
\hline & Time 2 & 3.76 & .41 & 3.11 & 1.05 \\
\hline \multirow{2}{*}{ Performance } & Time 1 & 34.06 & 9.39 & 6.69 & 2.66 \\
\hline & Time 2 & 15.69 & 3.18 & 7.2 & 2.76 \\
\hline
\end{tabular}


Table 4.4 presents the means and standard deviations of the answers in mother's and children's questionnaires. It seems that the mothers of this study had high levels of selfefficacy (highest score was 10 points) and that they used more authoritative parenting practices to raise their children. Considering children's temperament most of the mothers gave higher ratings in children's Effortful control, followed by Surgency/extraversion and lastly Negative affectivity.

Table 4.4

Descriptive Statistics for Mothers' and Children's Scales (Time 1)

Measures

\begin{tabular}{|c|c|c|c|}
\hline Scales & & $M$ & $S D$ \\
\hline $\begin{array}{l}\text { Tool of Parenting } \\
\text { Self-efficacy }\end{array}$ & Self-efficacy & 8.2 & .62 \\
\hline \multirow{4}{*}{$\begin{array}{c}\text { The Parenting Styles } \\
\text { and Dimensions } \\
\text { Questionnaire } \\
\text { (PSDQ) }\end{array}$} & $\begin{array}{c}\text { Authoritarian } \\
\text { mother }\end{array}$ & 1.76 & .43 \\
\hline & $\begin{array}{c}\text { Authoritative } \\
\text { mother }\end{array}$ & 4.28 & .38 \\
\hline & $\begin{array}{l}\text { Permissive } \\
\text { mother }\end{array}$ & 2.67 & .81 \\
\hline & $\begin{array}{l}\text { Strict } \\
\text { mother }\end{array}$ & 3.93 & .54 \\
\hline \multirow{3}{*}{$\begin{array}{c}\text { Children's } \\
\text { Behaviour } \\
\text { Questionnaire-Very } \\
\text { Short Form (CBQ- } \\
\text { VSF) }\end{array}$} & $\begin{array}{l}\text { Negative } \\
\text { affectivity }\end{array}$ & 4.20 & 1.09 \\
\hline & $\begin{array}{l}\text { Surgency/ } \\
\text { extraversion }\end{array}$ & 4.99 & 1.19 \\
\hline & $\begin{array}{l}\text { Effortful } \\
\text { control }\end{array}$ & 5.54 & .65 \\
\hline
\end{tabular}

Aggregate scores for maternal behaviours and children's SRL skills. For statistical reasons, it was decided to reduce the number of variables by merging some of them into broader meaningful dimensions. Therefore, for the maternal supportive behaviours, three new variables were computed. Specifically, the eight categories of maternal supportive behaviours were grouped into three mean scores. The first represented the Cognitive and Metacognitive support, and it was the mean of the cognitive score and the three metacognitive scores (Planning, Use of strategies and techniques and Monitoring of the progress and self-evaluation of the cognitive outcome). The second aggregate score 
represented the Emotional and Motivational support, and it was based on the scores of the Positive affective and motivational context, and Negative criticism-rejection (reversed scoring). The third mean score was calculated by the scores of Boosting the child's autonomy - active involvement and maternal Overcontrolling (reversed scoring), and it was labelled as Autonomy support.

In the same way, children's six SRL skills were reduced to three new computed variables. First, Cognitive and Metacognitive skills, which was the mean of the cognitive skills and the other two metacognitive skills (Planning of the solution process, and Selfmonitoring during problem-solving). Second, Emotional and Motivational skills, which included children's Concentration/interest and Persistence in the face of difficulties, and third, Autonomy skills, which referred to children's Working autonomously behaviour. Table 4.5 shows the descriptive statistics of the new aggregate scores for the maternal supportive behaviours and children's SRL skills.

Table 4.5

Descriptive Statistics for Aggregate Scores of the Measures

\begin{tabular}{|c|c|c|c|c|c|}
\hline & \multirow[t]{2}{*}{ Time } & \multicolumn{2}{|c|}{ Visual-spatial tasks } & \multicolumn{2}{|c|}{ Language tasks } \\
\hline & & $M$ & $S D$ & $M$ & $S D$ \\
\hline \multicolumn{6}{|l|}{ Mothers' measures } \\
\hline \multirow{2}{*}{ Maternal Contingency } & Time 1 & 2.38 & .58 & 2.00 & .59 \\
\hline & Time 2 & 2.22 & .61 & 1.83 & .78 \\
\hline \multirow{2}{*}{$\begin{array}{c}\text { Cognitive and } \\
\text { Metacognitive support }\end{array}$} & Time 1 & 2.53 & .56 & 2.08 & .72 \\
\hline & Time 2 & 3.02 & .47 & 2.56 & .69 \\
\hline \multirow{2}{*}{$\begin{array}{c}\text { Emotional and } \\
\text { Motivational support }\end{array}$} & Time 1 & 2.83 & .54 & 2.75 & .59 \\
\hline & Time 2 & 2.68 & .73 & 2.41 & .71 \\
\hline \multirow{2}{*}{ Autonomy support } & Time 1 & 2.63 & .60 & 2.58 & .80 \\
\hline & Time 2 & 2.87 & .70 & 2.6 & .96 \\
\hline \multicolumn{6}{|l|}{ Children's measures } \\
\hline Cognitive and & Time 1 & 2.78 & .73 & 1.97 & .85 \\
\hline Metacognitive skills & Time 2 & 3.26 & .59 & 2.47 & .78 \\
\hline \multirow{2}{*}{$\begin{array}{c}\text { Emotional-Motivational } \\
\text { skills }\end{array}$} & Time 1 & 3.35 & .54 & 2.68 & .73 \\
\hline & Time 2 & 3.44 & .51 & 2.54 & .78 \\
\hline \multirow{2}{*}{ Autonomy skills } & Time 1 & 3.59 & .49 & 2.73 & .78 \\
\hline & Time 2 & 3.76 & .41 & 3.11 & 1.05 \\
\hline \multirow{2}{*}{ Performance } & Time 1 & 34.06 & 9.39 & 6.69 & 2.66 \\
\hline & Time 2 & 15.69 & 3.18 & 7.2 & 2.76 \\
\hline
\end{tabular}




\subsubsection{Testing for Normality}

To confirm if the data of our study were appropriate for parametric tests, normal distribution was checked to decide if the mean score could be trusted or non-parametric tests should be used. Since the sample in this study was under 50, the Shapiro-Wilk test was used to check for the normality of the data.

The results indicated that some of the study's variables showed inconsistencies in the normal distribution. Specifically, the three aggregate scores of maternal supportive behaviours were normally distributed, except for Autonomy support in the Language tasks at Time 1, and Autonomy support in the Visual-spatial and Language tasks at Time 2. Maternal Contingency scores were not normally distributed at both times and tasks. Regarding children's scores, all were not normally distributed except for Emotional and Motivational skills in the Language tasks at Time 1 and children's performance in the Visual-spatial tasks at Time 1.

Because parametric tests rely on the mean to detect potential differences in one variable according to another, scores that have not normal distribution may distort their means. Therefore, the use of non-parametric tests, which are not based on mean scores, is preferred. For the above reasons, non-parametric tests were implemented in this study as well as the Bootstrap method in the partial correlation and multiple regression analysis. In bootstrapping "The original sample of size $n$ is treated as a miniature representation of the population originally sampled. Observations in this sample are then "resampled" with replacement, and some statistic of interest is calculated in the new sample of size $n$ constructed through this resampling process. Repeated over and over -thousands of times ideally- a representation of the sampling distribution of the statistic is constructed empirically, and this empirical representation is used for the inferential task at hand" (Hayes, 2018, p. 98).

Bootstrapping is only available for Entry methods in regression analysis in SPSS. As we explained above, some of the study's variables were not normally distributed; therefore, the bias-corrected and accelerated confidence intervals (BCaCI) in partial correlation, regression and mediation analyses were also reported. A genuine effect is likely to exist when this range does not include zero (Field, 2013). Confidence intervals are "a pair of values that define a range within which we expect a population parameter, such as the mean, to fall. In the case of $95 \%$ confidence interval, these values define the range within which there is a 95\% probability that the parameter will fall” (Brace, Kemp, \& Snelgar, 2016, p. 397). 
To investigate whether the mothers of this study were rated to use a particular type of support more or less frequently, as well as to explore if children were rated to employ more or less a particular SRL skill, within-subject ANOVAs and the Friedman Test (nonparametric data) were chosen. To examine possible relations between two variables, Spearman's correlation was used. In addition, Wilcoxon signed-rank test was employed to explore whether there are differences in the measures of a dependent variable (nonparametric) across two conditions of an independent variable (e.g., Cognitive support at Time 1 and Cognitive support at Time 2 or Cognitive support in the Visual-spatial tasks and Cognitive support in the Language task). For the variables with normal distribution, the related t-test was used. 


\subsection{Differences in Maternal Measures and Differences in Children's Measures}

\subsubsection{Maternal supportive behaviours}

To investigate the possible differences between the different types of maternal supportive behaviours, within-subject ANOVAs and the Friedman Test (for non-parametric data) were chosen. The results for Time 1 showed that there were overall significant differences between the three different scores in both cognitive domains. For the Visualspatial tasks, the multivariate effect was: Pillai's trace $=.222, F(2,33)=4.711, p<.05$, partial $\eta^{2}=.222$. The test of within-subject effects was: $F(2,68)=3.666, p<.05$, partial $\eta^{2}=$ .097. The pairwise comparisons were: Cognitive and Metacognitive support $(M=2.53)$ and Emotional and Motivational support $(M=2.83), p<.05$; Cognitive and Metacognitive support $(M=2.53)$ and Autonomy support, $(M=2.63), p>.05$; Emotional and Motivational support $(M=2.83)$ and Autonomy support, $(M=2.63), p>.05$. The results support that mothers were rated significantly higher in Emotional and Motivational support than Cognitive and Metacognitive support in the Visual-spatial tasks at Time 1.

For the Language task, the Friedman Test showed that there was a statistically significant difference in the maternal type of support $\left[x^{2}(2)=8.785, p<.05\right]$. To detect which of the variables differed, paired t-tests were run for the parametric data and Post hoc analysis with Wilcoxon signed-rank tests were used for the non-parametric data. Paired t-tests showed that there were statistically significant differences between maternal Cognitive and Metacognitive support and maternal Emotional and Motivational support $[t(34)=-4.547, p<$ $.001]$, with mothers being rated higher in Emotional and Motivational support compared to Cognitive and Metacognitive support. Post hoc analysis with Wilcoxon signed-rank tests indicated that one more statistically significant difference occurred between maternal Cognitive and Metacognitive support and maternal Autonomy support $(Z=-2.158, p<.05)$, with mothers offering their children more Autonomy support than Cognitive and Metacognitive support. There were no statistically significant differences between maternal Emotional and Motivational support and maternal Autonomy support $(Z=-1.206, p>.05)$.

The results for Time 2 indicated that there was a statistically significant difference in the type of support but only in the Visual-spatial tasks. For the Language task, $x^{2}(2)=1.851$, $\mathrm{p}>.05$; and for the Visual-spatial tasks, $x^{2}(2)=4.887, p=.05$. Thus, for the Visual-spatial tasks, a paired t-test showed that there was a statistically significant difference between maternal Cognitive and Metacognitive support and maternal Emotional and Motivational 
support $[t(34)=2.833, p<.01]$, with mothers being rated higher in Cognitive and Metacognitive support compared to Emotional and Motivational support. For the nonparametric variables, post hoc analysis with Wilcoxon signed-rank tests was conducted. There were no statistically significant differences between maternal Cognitive and Metacognitive support and maternal Autonomy support $(Z=-1.488, p>.05)$ as well as between maternal Emotional and Motivational support and maternal Autonomy support $(Z=$ $-1.736, p>.05)$.

In general, the above results support that at Time 1 mothers were rated higher in Emotional and Motivational behaviours in their efforts to help their children during problemsolving both in the Visual-spatial and Language tasks. However, at Time 2, mothers were rated higher in providing Cognitive and Metacognitive support in comparison to Emotional and Motivational support, especially in the Visual-spatial tasks.

\subsubsection{Children's SRL skills}

To investigate the possible differences between the different groups of children's SRL skills, the Friedman Test (for non-parametric data) was chosen. For the Visual-spatial tasks at Time 1, the Friedman Test showed that there was a statistically significant difference in children's use of SRL skills $\left[x^{2}(2)=51.063, p<.001\right]$. Post hoc analysis with Wilcoxon signed-rank tests was conducted. There was a statistically significant difference between the use of Cognitive and Metacognitive skills and Emotional and Motivational skills $(Z=-5.047$, $p<.001)$. The results showed that children's Cognitive-Metacognitive skills differed significantly from their Autonomy skills $(Z=-5.032, p<.001)$. One more difference was noticed between children's Emotional-Motivational skills and children's Autonomy skills ( $Z$ $=-2.879, p<.01)$. The above results suggest that children demonstrated significantly more Autonomy skills than Emotional-Motivational and Cognitive-Metacognitive skills as well as more Emotional-Motivational skills than Cognitive-Metacognitive skills in the Visual-spatial tasks at Time 1.

In the Language tasks at Time 1, the Friedman Test showed that there was a statistically significant difference between children's use of SRL skills $\left[x^{2}(2)=37.070, p<\right.$ .001]. Post hoc analysis with Wilcoxon signed-rank tests showed that there was a statistically significant difference in the use of Cognitive-Metacognitive skills and EmotionalMotivational skills $(Z=-4.805, p<.001)$. The results also confirmed that children's Cognitive-Metacognitive skills differed significantly from their Autonomy skills $(Z=-4.611$, 
$p<.001)$. There was no statistically significant difference between children's EmotionalMotivational skills and children's Autonomy skills $(Z=-.634, p>.05)$. The above results show that children were rated significantly higher in Autonomy skills than in CognitiveMetacognitive skills as well as more Emotional-Motivational skills than CognitiveMetacognitive skills in the Language tasks at Time 1.

For the Visual-spatial tasks at Time 2, the Friedman Test revealed that there was a statistically significant difference between children's use of SRL skills $\left[x^{2}(2)=37.967, p<\right.$ .001]. Post hoc analysis with Wilcoxon signed-rank tests was conducted and showed that there was a statistically significant difference in the use of Cognitive-Metacognitive skills and Emotional-Motivational skills $(Z=-3.449, p<.001)$. The results also provided evidence that children's Cognitive-Metacognitive skills differed significantly from their Autonomy skills $(Z=-4.418, p<.001)$. One more difference was noticed between children's EmotionalMotivational skills and children's Autonomy skills $(Z=-3.775, p<.001)$. The above results indicate that children employed significantly more Autonomy skills than EmotionalMotivational skills and Cognitive-Metacognitive skills as well as more EmotionalMotivational skills than Cognitive-Metacognitive skills.

In the Language tasks at Time 2, the Friedman Test showed that there was a statistically significant difference between children's use of SRL skills $\left[x^{2}(2)=27.232, p<\right.$ .001)]. Post hoc analysis with Wilcoxon signed-rank tests showed that there was no statistically significant difference in the use of Cognitive and Metacognitive skills and Emotional and Motivational skills $(Z=-1.132, p>.05)$. However, the results revealed that children's Cognitive and Metacognitive skills differed significantly from their Autonomy skills $(Z=-3.909, p<.001)$. There was also a statistically significant difference between children's Emotional and Motivational skills and children's Autonomy skills $(Z=-3.855, p<$ $.01)$. The above results imply that children were rated significantly higher in Autonomy skills than in Cognitive-Metacognitive skills and Emotional-Motivational skills.

Taken together, these results suggest that mothers were rated higher in Emotional and Motivational support and lower in Cognitive and Metacognitive support at Time 1, whereas six months later (Time 2), they were rated higher in Cognitive and Metacognitive support and lower in Emotional and Motivational support. On the other hand, children's use of SRL skills were recorded as more stable: that is, Autonomy and Emotional-Motivational skills were recorded higher than the Cognitive-Metacognitive skills at both research times. 


\subsection{Children's and Mothers' Demographic Variables and Characteristics}

One of the aims of this thesis was to examine possible demographic factors and characteristics of mothers and children that might relate to the supportive behaviours of mothers and children's SRL skills. These factors included demographic information, such as maternal education, children's sex and age as well as information regarding aspects of the children's temperament or parenting. For the parametric data of this study, independent sample T-Tests were run to contrast the means between two independent groups on the same dependent variable. If the dependent variable was not normally distributed for each group of the independent variable, then Mann-Whitney U tests were used. Spearman's correlations were also conducted to discover possible associations between the demographic information (e.g., children's age), the scales' data (e.g., children's temperament) and maternal supportive and contingent behaviours as well as children's SRL skills and performance.

\subsubsection{Demographic variables: Associations with maternal supportive and contingent behaviours, children's SRL skills and performance}

Maternal education. Mothers' education was allocated in three groups; the first group included mothers with a secondary education degree, the second group was comprised by mothers with a bachelor's degree, and the last group consisted of mothers who held a postgraduate degree. To examine if the education of the mothers in this study was associated with maternal support or children's SRL skills, Spearman's correlations were conducted.

Table 4.6 presents the correlations between maternal education and maternal supportive and contingent behaviours as well as the correlations between maternal education and children's SRL skills and performance, in the Visual-spatial and Language tasks, at both research times. The results showed no evidence of associations between maternal education and the dimensions of maternal behaviours and this outcome was consistent for the two types of tasks and the two research times. An interesting result was found regarding the correlations between maternal education and children's SRL skills and performance. Maternal education was significantly and positively correlated with most of the children's skills and performance, but only at Time 1 and for both types of tasks. 
Table 4.6

Spearman's Correlations between Maternal Education, Maternal Supportive and Contingent Behaviours and Children's SRL skills and Performance in the Visual-spatial and Language Tasks

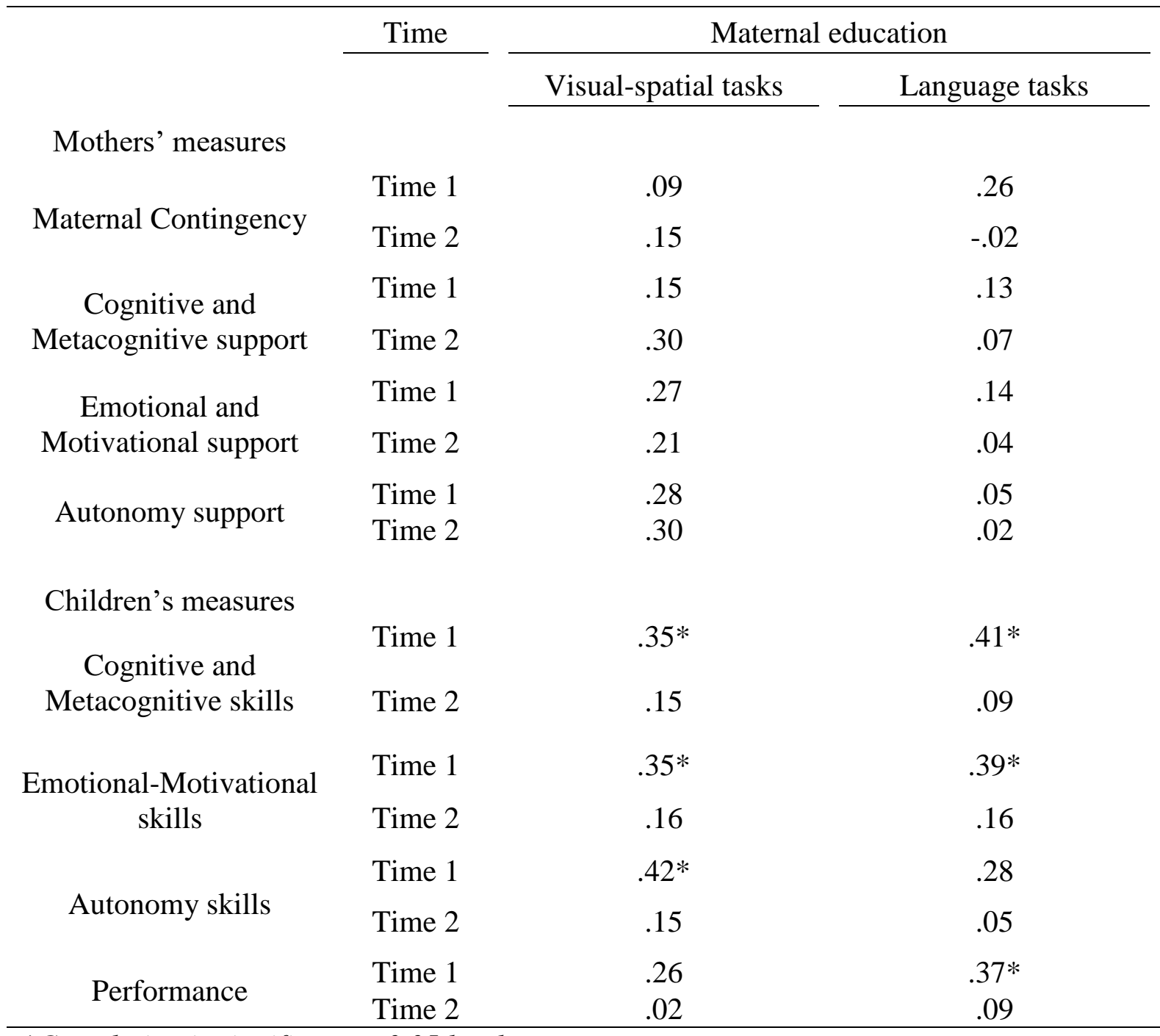

*Correlation is significant at 0.05 level

Children's age. Spearman's correlations were also conducted to explore associations between children's age (measured as a continuous variable) and maternal supportive and contingent behaviours as well as between children's age and children's SRL skills and performance. Children's age at Time 1 ranged from 54 to 72 months and at Time 2 from 59 to 77 months. The results are presented in table 4.7 and indicate that children's age was significantly associated with maternal Contingency in both types of tasks at Time 1, and in the Visual-spatial tasks at Time 2. Children's age also showed a significant correlation with maternal Autonomy in the Visual-spatial tasks at Time 1. The results suggest that the mothers 
of the older children were rated higher in providing Contingent behaviours in their interaction with their child.

The table also shows that children's age was significantly associated with almost all of the children's SRL skills and performance at both types of tasks and at the two research times. In other words, the older children of this study were rated higher in their SRL skills and had better performance than the younger children, and this was consistent with both research times and type of tasks.

Table 4.7

Spearman's Correlations between Children's Age, Maternal Supportive and Contingent Behaviours and Children's SRL skills and Performance in the Visual-spatial and Language Tasks

\begin{tabular}{|c|c|c|c|}
\hline & \multirow[t]{2}{*}{ Time } & \multicolumn{2}{|c|}{ Children's age } \\
\hline & & Visual-spatial tasks & Language tasks \\
\hline \multicolumn{4}{|l|}{ Mothers' Measures } \\
\hline \multirow{2}{*}{ Maternal Contingency } & Time 1 & $.46 * *$ & $.38 *$ \\
\hline & Time 2 & $.34^{*}$ & .22 \\
\hline \multirow{2}{*}{$\begin{array}{c}\text { Cognitive and } \\
\text { Metacognitive support }\end{array}$} & Time 1 & -.04 & .25 \\
\hline & Time 2 & .22 & -.15 \\
\hline \multirow{2}{*}{$\begin{array}{c}\text { Emotional and } \\
\text { Motivational support }\end{array}$} & Time 1 & .06 & .14 \\
\hline & Time 2 & .07 & .07 \\
\hline \multirow{2}{*}{ Autonomy support } & Time 1 & $.61 * * *$ & .09 \\
\hline & Time 2 & .27 & .25 \\
\hline \multicolumn{4}{|l|}{ Children's measures } \\
\hline \multirow{2}{*}{$\begin{array}{c}\text { Cognitive and } \\
\text { Metacognitive skills }\end{array}$} & Time 1 & $.62 * * *$ & $.51 * *$ \\
\hline & Time 2 & $.43 * *$ & $.41 *$ \\
\hline \multirow{2}{*}{$\begin{array}{l}\text { Emotional-Motivational } \\
\text { skills }\end{array}$} & Time 1 & $.56^{* *}$ & .31 \\
\hline & Time 2 & $.36^{*}$ & $.49 * *$ \\
\hline \multirow{2}{*}{ Autonomy skills } & Time 1 & .33 & .19 \\
\hline & Time 2 & .17 & $.38^{*}$ \\
\hline \multirow{2}{*}{ Performance } & Time 1 & $.60 * * *$ & $.44 * *$ \\
\hline & Time 2 & .31 & $.43 *$ \\
\hline
\end{tabular}

Children's gender. In order to test if children's gender differentiates maternal contingent and supporting behaviours, children's SRL skills and performance, Independent $\mathrm{T}$-Tests were used, if the dependent variable was approximately normally distributed for each 
group of the independent variable, and Mann-Whitney $U$ tests were used in the case of no normal distribution.

For maternal supportive and contingent behaviours in the Visual-spatial tasks (Time 1) the results were not statistically significant. Specifically, for maternal Contingency, $U=114.000, Z=-.870, p>.05$; for Cognitive and Metacognitive support, $t(33)=-.154, p>.05$; for Emotional and Motivational support, $t(33)=1.490, p>.05$; and for Autonomy support, $t(33)=-.045, p>.05$. For maternal supportive and contingent behaviours in the Language tasks (Time 1) the findings were not statistically significant. In particular, the results showed that for maternal Contingency, $U=109.000, Z=-1.199, p>.05$; for Cognitive and Metacognitive support, $t(33)=-.412, p>.05$; for Emotional and Motivational support,,$t(33)$ $=.897 . p>.05$; and for Autonomy support, $U=112.000, Z=-.922, p>.05$.

For maternal supportive and contingent behaviours in the Visual-spatial tasks (Time 2) no statistically significant differences were revealed. For Cognitive and Metacognitive support, $t(33)=.743, p>.05$; for Emotional and Motivational support, $t(33)=-.745, p>.05$; for Autonomy support, $U=105.500, Z=-1.135, p>.05$; and for maternal Contingency, $U=$ $96.500, Z=-1.464, \mathrm{p}>.05$. For maternal supportive and contingent behaviours in the Language tasks (Time 2) statistically significant results were found only for maternal Contingency, $U=49.500, Z=-3.291, p<.01$ (Median for boys $=1.00$ and Median for girls = 2.00), effect size $r=.555$; and for Autonomy support $U=81.000, Z=-2.021, p<.05$ (Median for boys $=2.00$ and Median for girls $=2.75$ ), effect size $r=.341$. For Cognitive and Metacognitive support, $t(33)=.393, p>.05$ and for Emotional and Motivational support, $t(33)=-1.824, p>.05$.

For children's SRL skills and performance the results, on the whole, were not statistically significant. In the Visual-spatial tasks (Time 1) Mann - Whitney U tests and Independent $\mathrm{T}$ test indicated that: for Cognitive and Metacognitive skills, $U=110.500, Z=-$ .957, $p>.05$; for Emotional and Motivational skills, $U=93.000, Z=-1.574, p>.05$; for Autonomy skills, $U=124.000, Z=-.533, p>.05$; and for children's performance $t(33)=-$ $.882, p>.05$. For children's SRL skills and performance in the Language tasks (Time 1) the results showed that: for Cognitive and Metacognitive skills, $U=137.000, Z=-.035, p>.05$; for Emotional and Motivational skills, $t(33)=-.908, p>.05$; for Autonomy skills, $U=$ $126.500, Z=-.421, p>.05$; and for children's performance $t(33)=-.168, p>.05$.

For children's SRL skills and performance in the Visual-spatial tasks (Time 2) the results showed that: for Cognitive and Metacognitive skills, $U=113.000, Z=-.871, p>.05$; for Emotional and Motivational skills, $U=129.000, Z=-.316, p>.05$; for Autonomy skills, 
$U=137.000, Z=-.042, p>.05$; and for children's performance, $t(33)=-1.334, p>.05$. For children's SRL skills and performance in the Language task (Time 2) the following were found: for Cognitive and Metacognitive skills, $U=108.000, Z=-1.073, p>.05$; for Emotional and Motivational skills, $t(33)=-1.140, p>.05$; for Autonomy skills, $U=117.000$, $Z=-.793, \mathrm{p}>.05$; and for children's performance, $U=101.000, Z=-1.199, \mathrm{p}>.05$.

In general, no significant differences were found regarding the use of maternal supportive and contingent behaviours and children's SRL skills and performance in boys and girls at both times and type of tasks. An exception was noticed regarding maternal Contingency and maternal Autonomy in the Language tasks at Time 2 where mothers were rated higher in their Contingent behaviours and Autonomy support to girls than boys, with medium (for Autonomy support) and strong effect size (for Contingency).

\subsubsection{Maternal and children's characteristics: Associations with maternal supportive and contingent behaviours, children's SRL skills and performance}

To investigate whether children's temperament, maternal parenting style and maternal self-efficacy were correlated with maternal supportive and contingent behaviours or children's SRL skills and performance, Spearman's correlation was conducted.

Tables 4.8 and 4.9 depict the correlations between maternal supportive and contingent behaviours, maternal self-efficacy, maternal parenting style and children's temperament in the Visual-spatial and Language tasks at both research times. The results indicate that none of the above factors were significantly correlated with the maternal dimensions, except for some random and scattered correlations. The results were consistent for both research times and both types of tasks.

Tables 4.10 and 4.11 illustrate Spearman's correlations between children's SRL skills and performance, maternal self-efficacy, maternal parenting style and children's temperament in the Visual-spatial and Language tasks at both research times. Maternal self-efficacy and children's temperament showed no significant correlations with children's SRL skills and performance. Peculiarly, from the maternal parenting styles, maternal authoritarian parenting was positively correlated with all of the children's SRL skills and performance in the Visualspatial tasks but only at Time 2, and negatively correlated with almost all of the children's SRL skills and performance in the Language tasks but only at Time 1. 
Table 4.8

Spearman's Correlations between Maternal and Children's Characteristics and Maternal Support in the Visual-spatial Tasks

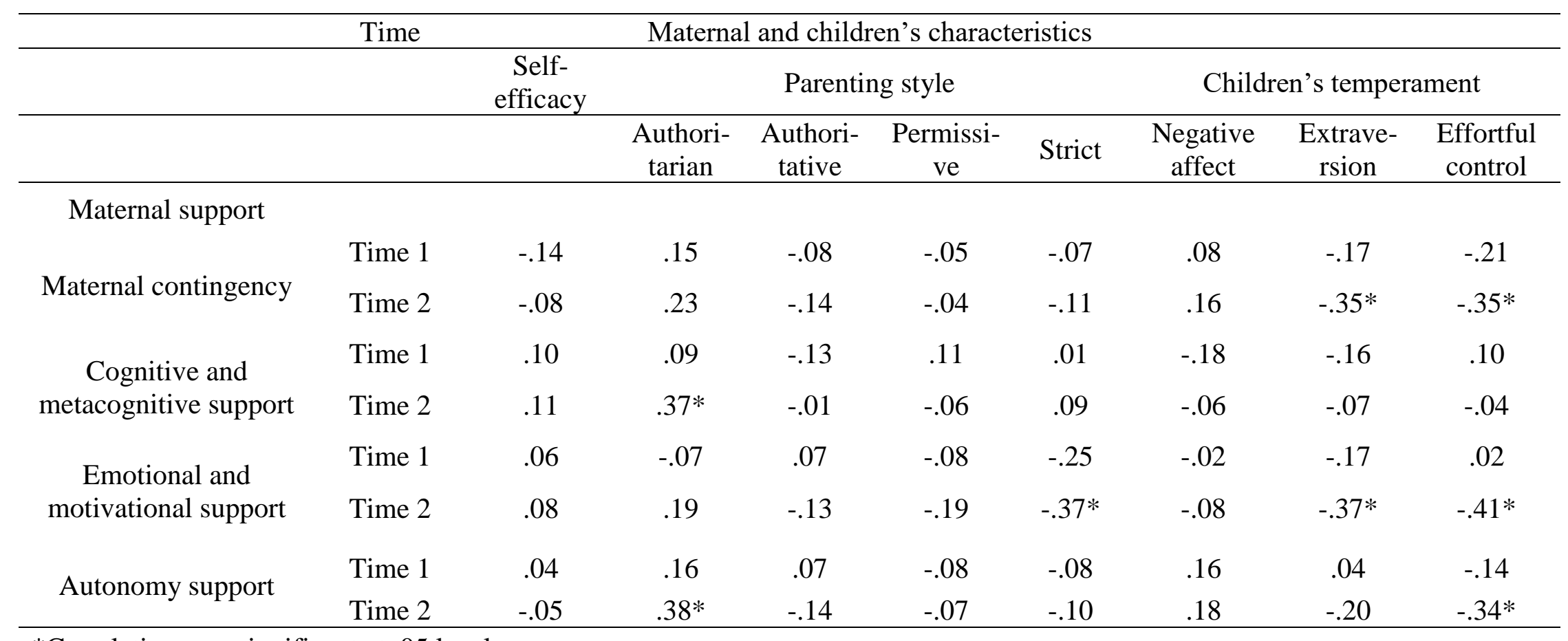

*Correlations are significant at .05 level 
Table 4.9

Spearman's Correlations between Maternal and Children's Characteristics and Maternal support in the Language Tasks

\begin{tabular}{|c|c|c|c|c|c|c|c|c|c|}
\hline \multicolumn{3}{|c|}{ Time } & \multicolumn{7}{|c|}{ Maternal and children's factors } \\
\hline & & \multirow[t]{2}{*}{$\begin{array}{c}\text { Self- } \\
\text { efficacy }\end{array}$} & \multicolumn{4}{|c|}{ Parenting style } & \multicolumn{3}{|c|}{ Children's temperament } \\
\hline & & & $\begin{array}{l}\text { Authori- } \\
\text { tarian }\end{array}$ & $\begin{array}{c}\text { Authori- } \\
\text { tative }\end{array}$ & $\begin{array}{l}\text { Permissi- } \\
\text { ve }\end{array}$ & Strict & $\begin{array}{l}\text { Negative } \\
\text { affect }\end{array}$ & $\begin{array}{l}\text { Extrave- } \\
\text { rsion }\end{array}$ & $\begin{array}{c}\text { Effortful } \\
\text { control }\end{array}$ \\
\hline \multicolumn{10}{|l|}{ Maternal support } \\
\hline \multirow[b]{2}{*}{ Maternal contingency } & Time 1 & .30 & -.10 & .14 & -.32 & -.07 & .16 & .03 & .15 \\
\hline & Time 2 & -.17 & .16 & -.08 & .18 & -.06 & .12 & -.23 & .01 \\
\hline \multirow{2}{*}{$\begin{array}{c}\text { Cognitive and } \\
\text { Metacognitive Support }\end{array}$} & Time 1 & .08 & .09 & .05 & -.02 & .11 & -.06 & .04 & .17 \\
\hline & Time 2 & .15 & -.05 & .05 & -.07 & -.11 & -.13 & .11 & .08 \\
\hline Emotional and & Time 1 & .27 & -.09 & .17 & -.26 & -.29 & -.01 & .14 & .27 \\
\hline Motivational Support & Time 2 & .04 & .21 & -.09 & .02 & -.17 & -.12 & $-.35^{*}$ & -.16 \\
\hline \multirow{2}{*}{ Autonomy support } & Time 1 & .23 & -.10 & .04 & -.23 & -.22 & .13 & -.19 & -.07 \\
\hline & Time 2 & -.14 & .23 & -.28 & .19 & .03 & .02 & -.20 & -.20 \\
\hline
\end{tabular}

*Correlations are significant at .05 level 
Table 4.10

Spearman's Correlations between Maternal and Children's Characteristics and Children's SRL Skills and Performance in the Visual-spatial Tasks

\begin{tabular}{|c|c|c|c|c|c|c|c|c|c|}
\hline \multicolumn{4}{|c|}{ Time } & \multicolumn{6}{|c|}{ Maternal and children's factors } \\
\hline & & \multirow[t]{2}{*}{$\begin{array}{c}\text { Self- } \\
\text { efficacy }\end{array}$} & \multicolumn{4}{|c|}{ Parenting style } & \multicolumn{3}{|c|}{ Children's temperament } \\
\hline & & & $\begin{array}{l}\text { Authori- } \\
\text { tarian }\end{array}$ & $\begin{array}{l}\text { Authori- } \\
\text { tative }\end{array}$ & Permissive & Strict & $\begin{array}{l}\text { Negative } \\
\text { affect }\end{array}$ & $\begin{array}{l}\text { Extrave- } \\
\text { rsion }\end{array}$ & $\begin{array}{c}\text { Effortful } \\
\text { control }\end{array}$ \\
\hline \multicolumn{10}{|l|}{$\begin{array}{l}\text { Children's skills and } \\
\text { performance }\end{array}$} \\
\hline \multirow{2}{*}{$\begin{array}{l}\text { Cognitive and } \\
\text { metacognitive skills }\end{array}$} & Time 1 & -.04 & .13 & .05 & -.11 & -.12 & $.39 *$ & -.07 & -.01 \\
\hline & Time 2 & .03 & $.39 *$ & .05 & -.10 & .08 & $.35^{*}$ & -.19 & -.12 \\
\hline \multirow{2}{*}{$\begin{array}{l}\text { Emotional and } \\
\text { motivational skills }\end{array}$} & Time 1 & .09 & -.01 & .05 & -.02 & -.13 & $.35^{*}$ & -.23 & .10 \\
\hline & Time 2 & .01 & $.39 *$ & -.06 & -.11 & -.04 & .20 & -.29 & -.12 \\
\hline \multirow{2}{*}{ Autonomy skills } & Time 1 & -.07 & .27 & -.14 & .04 & -.14 & .20 & -.22 & -.21 \\
\hline & Time 2 & .03 & $.43^{*}$ & .04 & -.21 & -.03 & .11 & -.02 & -.13 \\
\hline \multirow{2}{*}{ Performance } & Time 1 & -.18 & $.37 *$ & -.02 & .05 & -.05 & .17 & -.00 & -.02 \\
\hline & Time 2 & -.04 & $.40^{*}$ & .02 & -.12 & .06 & .16 & -.22 & -.25 \\
\hline
\end{tabular}

*Correlations are significant at .05 level 
Table 4.11

Spearman's Correlations between Maternal and Children's Characteristic and Children's SRL Skills and Performance in the Language Tasks

\begin{tabular}{|c|c|c|c|c|c|c|c|c|c|}
\hline \multicolumn{4}{|c|}{ Time } & \multicolumn{6}{|c|}{ Maternal and children's factors } \\
\hline & & \multirow[t]{2}{*}{$\begin{array}{c}\text { Self- } \\
\text { efficacy }\end{array}$} & \multicolumn{4}{|c|}{ Parenting style } & \multicolumn{3}{|c|}{ Children's temperament } \\
\hline & & & $\begin{array}{c}\text { Authorita } \\
\text { rian }\end{array}$ & $\begin{array}{c}\text { Authori- } \\
\text { tative }\end{array}$ & Permissive & Strict & $\begin{array}{l}\text { Negative } \\
\text { affect }\end{array}$ & $\begin{array}{c}\text { Extrave- } \\
\text { rsion }\end{array}$ & $\begin{array}{c}\text { Effortful } \\
\text { control }\end{array}$ \\
\hline \multicolumn{10}{|l|}{$\begin{array}{l}\text { Children's skills and } \\
\text { performance }\end{array}$} \\
\hline \multirow{2}{*}{$\begin{array}{l}\text { Cognitive and } \\
\text { metacognitive skills }\end{array}$} & Time 1 & .18 & -.29 & .17 & .02 & .00 & .28 & .21 & .24 \\
\hline & Time 2 & -.02 & -.08 & .01 & .16 & -.04 & .12 & .03 & .02 \\
\hline \multirow{2}{*}{$\begin{array}{l}\text { Emotional and } \\
\text { motivational skills }\end{array}$} & Time 1 & $.40^{*}$ & $-.40^{*}$ & .28 & -.09 & -.10 & .04 & -.05 & .19 \\
\hline & Time 2 & -.07 & -.04 & -.00 & .12 & -.08 & .07 & -.01 & .02 \\
\hline \multirow{2}{*}{ Autonomy skills } & Time 1 & .31 & $-.39 *$ & .21 & -.01 & -.05 & .18 & -.03 & .12 \\
\hline & Time 2 & .04 & -.18 & -.02 & .05 & -.03 & -.08 & -.02 & -.00 \\
\hline \multirow{2}{*}{ Performance } & Time 1 & .19 & $-.42 *$ & .19 & .05 & -.14 & .22 & .11 & .17 \\
\hline & Time 2 & -.07 & -.04 & .01 & .11 & -.01 & .08 & .15 & .04 \\
\hline
\end{tabular}

*correlations are significant at .05 level 


\subsection{Results from Time 1}

\subsubsection{Maternal supportive and contingent behaviours and children's SRL skills and performance (Time 1)}

To investigate whether the maternal dimensions are associated with children's SRL skills and performance, partial correlations were conducted controlling for children's age. For these analyses, the bootstrap method was used reporting the 95\% bias-corrected and accelerated confidence intervals for B in a parenthesis (BCa CI), based on 1000 samples.

Table 4.12 depicts the correlations between maternal supportive and contingent behaviours and children's skills. What is apparent from the table is that two of the maternal dimensions (maternal Contingency and maternal Autonomy support) were significantly associated with most of the children's SRL skills and performance. Maternal Cognitive and Metacognitive support, as well as maternal Emotional and Motivational support, showed no significant correlations with children's measures. For both types of tasks, maternal Autonomy support was positively and significantly associated with all of the children's skills. Maternal Contingency was significantly and positively associated with all of the children's skills and performance in the Language task, and with children's Cognitive and Metacognitive skills in the Visual-spatial tasks. The correlations were moderate to high.

Based on the correlations found above, multiple regression analyses with the Enter method and the use of the bootstrap method were implemented to test how much of the variance in the children's skills scores was explained by the maternal supportive and contingent behaviours which presented significant correlations with them. The bootstrap method was used reporting the $95 \%$ bias-corrected and accelerated confidence intervals for B (BCa CI), based on 1000 samples. Due to the small number of participants in this study, the maximum number of predictors for each regression model was three (those with highest correlation with the outcome variable), following the rule of thumb that there should be at least ten times as many participants as predictor variables (Brace, Kemp, \& Snelgar, 2016).

Table 4.13 presents the multiple regression analyses for all of the children's skills and performance in the Visual-spatial tasks at Time 1. For children's Cognitive and Metacognitive skills, maternal Contingency and children's age significantly contributed to the model which predicted $57.7 \%$ of its variance, and it was statistically significant $F(3,31)=$ 14.113, $p<.001$. However, even though the bias-corrected and accelerated confidence intervals for B concerning maternal Contingency and children's age did not contain zero, the 
$p$ values of the confidence intervals were .068 and .056 respectively, which yields concerns about the genuineness of the result. The second model was statistically significant in predicting $44.3 \%$ of the variance of children's Emotional and Motivational skills, but only maternal Autonomy support independently contributed to the model $[F(3,31)=8.205, p<$ .001]. However, even though the bias-corrected and accelerated confidence intervals for B concerning maternal Autonomy support did not contain zero, the $p$ value of the confidence intervals were .062 which yields some serious concerns about the genuineness of the result. The third model indicates that only maternal Autonomy support was an independent predictor of children's Autonomy skills. The model was able to predict $30.7 \%$ of the outcome variance $[F(3,31)=4.581, p<.01]$. Finally, in the last model, none of the predictors contributed independently to children's performance. Nonetheless, the model was statistically significant $[F(3,31)=7.172, p<.01]$. There were strong effect sizes for the first, second and last regression outcome and a moderate effect size for the third regression outcome $\left(f^{2}=1.15, f^{2}=\right.$ $0.64, f^{2}=0.31, f^{2}=0.54$ respectively).

Collinearity statistics were checked for possible problems with multicollinearity. The lowest Tolerance level was .465, and the highest variance inflation factor (VIF) was 2.152 for the first and last model. The lowest Tolerance level was .587, and the highest variance inflation factor (VIF) was 1.704 for the second and third model. Tolerance values can vary between 0 and 1 . Tolerance values closer to zero and a large value of VIF indicate problems with multicollinearity (Brace, Kemp, \& Snelgar, 2016). Thus, no problems with multicollinearity were detected.

In the same line, table 4.14 portrays the multiple regression analyses for the Language tasks at Time 1. Based on the correlations between maternal supportive and contingent behaviours and children's SRL skills and performance, the predictors entered to the model were three of the following variables: maternal Contingency, maternal Autonomy support, children's age and maternal education. The results indicated that all models were statistically significant at predicting each one of children's SRL skills and performance. For children's Cognitive and Metacognitive skills, maternal Contingency significantly contributed to the model which predicted $46.1 \%$ of its variance, and it was statistically significant $F(3,31)=$ $8.855, p<.001$. For the second model, although it was statistically significant at predicting children's Emotional and Motivational skills $[F(3,31)=5.541, p<.01]$, none of the predictor variables contributed independently. In the same line, none of the predictors independently contributed to the third model, yet it was statistically significant $[F(2,32)=6.670, p<.01]$. Considering children's performance, the overall model was statistically significant $[F(3,31)$ 
$=7.265, p<.01]$ and predicted $41.3 \%$ of the outcome variance. Maternal Autonomy and children's age were independent predictors of children's performance. There were strong effect sizes for the first, second and last regression outcomes and a moderate effect size for the third regression outcome, $\left(f^{2}=0.69, f^{2}=0.40, f^{2}=0.33, f^{2}=0.55\right.$ respectively $)$.

The lowest Tolerance level was .799, and the highest variance inflation factor (VIF) was 1.252 for the first model. The lowest Tolerance level was .731 , and the highest variance inflation factor (VIF) 1.368 for the second model. The lowest Tolerance level was .784, and the highest variance inflation factor (VIF) was 1.275 for the third model. The lowest Tolerance level was .853, and the highest variance inflation factor (VIF) was 1.172 for the last model, indicating no concerns of multicollinearity.

Summarizing the results for Time 1, it seems that maternal Contingency and maternal Autonomy were significantly correlated with most of the children's SRL skills and performance in both types of tasks. In addition, the multiple regression analyses showed that for children's Cognitive and Metacognitive skills, maternal Contingency and children's age were the most important predictors in the Visual-spatial tasks (this finding should be interpreted with caution, also taking into account the results of the bootstrap for coefficients) while maternal Contingency was the most important predictor in the Language task. For children's Emotional and Motivational skills, maternal Autonomy support was an independent predictor but only for the Visual-spatial tasks (this finding should be interpreted with caution, also taking into account the results of the bootstrap for coefficients). For children's Autonomy skills, maternal Autonomy support was an independent predictor but only in the Visual-spatial tasks. Finally, children's performance was significantly predicted by maternal Autonomy and children's age but only in the Language task. 


\section{Table 4.12}

Partial Correlations between Maternal Support and Children's Skills and Performance in the Visual-spatial and Language Tasks (T1), Controlling for Children's Age

\begin{tabular}{|c|c|c|c|c|c|c|c|c|}
\hline & \multicolumn{6}{|c|}{ Children's SRL skills } & \multicolumn{2}{|c|}{ Performance } \\
\hline & \multicolumn{2}{|c|}{$\begin{array}{c}\text { Cognitive and Metacognitive } \\
\text { skills }\end{array}$} & \multicolumn{2}{|c|}{$\begin{array}{c}\text { Emotional and Motivational } \\
\text { skills }\end{array}$} & \multicolumn{2}{|c|}{$\begin{array}{l}\text { Autonomy } \\
\text { skills }\end{array}$} & \multirow[b]{2}{*}{$\begin{array}{c}\text { Visual- } \\
\text { spatial tasks } \\
\end{array}$} & \multirow[b]{2}{*}{$\begin{array}{c}\text { Language } \\
\text { tasks }\end{array}$} \\
\hline & $\begin{array}{c}\text { Visual-spatial } \\
\text { tasks } \\
\end{array}$ & $\begin{array}{c}\text { Language } \\
\text { tasks }\end{array}$ & $\begin{array}{c}\text { Visual-spatial } \\
\text { tasks } \\
\end{array}$ & $\begin{array}{c}\text { Language } \\
\text { tasks }\end{array}$ & $\begin{array}{c}\text { Visual-spatial } \\
\text { tasks } \\
\end{array}$ & $\begin{array}{c}\text { Language } \\
\text { tasks }\end{array}$ & & \\
\hline \multicolumn{9}{|l|}{ Maternal support } \\
\hline $\begin{array}{c}\text { Maternal } \\
\text { Contingency }\end{array}$ & $\begin{array}{c}.51 * * \\
(0.21,0.76)\end{array}$ & $\begin{array}{c}.45^{* *} \\
(0.17,0.68)\end{array}$ & $\begin{array}{c}.31 \\
(0.03,0.62)\end{array}$ & $\begin{array}{c}.41^{*} \\
(0.03,0.67)\end{array}$ & $\begin{array}{c}.24 \\
(-0.14,0.6)\end{array}$ & $\begin{array}{c}.44 * * \\
(0.10,0.68)\end{array}$ & $\begin{array}{c}.33 \\
(0.001,0.62)\end{array}$ & $\begin{array}{c}.37 * \\
(0.10,0.60)\end{array}$ \\
\hline $\begin{array}{l}\text { Cognitive and } \\
\text { Metacognitive } \\
\text { support }\end{array}$ & $\begin{array}{c}-.10 \\
(-0.54,0.43)\end{array}$ & $\begin{array}{c}.13 \\
(-0.16,0.43)\end{array}$ & $\begin{array}{c}.06 \\
(-0.44,0.55)\end{array}$ & $\begin{array}{c}-.11 \\
(-0.42,0.19)\end{array}$ & $\begin{array}{c}.04 \\
(-0.37,0.44)\end{array}$ & $\begin{array}{c}-.23 \\
(-0.54,0.07)\end{array}$ & $\begin{array}{c}.09 \\
(-0.3,0.5)\end{array}$ & $\begin{array}{c}.04 \\
(-0.23,0.30)\end{array}$ \\
\hline $\begin{array}{c}\text { Emotional and } \\
\text { Motivational support }\end{array}$ & $\begin{array}{c}.22 \\
(-0.14,0.60)\end{array}$ & $\begin{array}{c}.04 \\
(-0.30,0.33)\end{array}$ & $\begin{array}{c}.18 \\
(-0.15,0.57)\end{array}$ & $\begin{array}{c}.06 \\
(-0.30,0.37)\end{array}$ & $\begin{array}{c}.29 \\
(-0.03,0.62)\end{array}$ & $\begin{array}{c}.02 \\
(-0.28,0.30)\end{array}$ & $\begin{array}{c}.23 \\
(-0.09,0.55)\end{array}$ & $\begin{array}{c}-.02 \\
(-0.35,0.32)\end{array}$ \\
\hline Autonomy support & $\begin{array}{c}.44 * * \\
(0.13,0.72)\end{array}$ & $\begin{array}{c}.36 * \\
(0.06,0.59)\end{array}$ & $\begin{array}{c}.36^{*} \\
(0.03,0.66)\end{array}$ & $\begin{array}{c}.37 * \\
(0.05,0.63)\end{array}$ & $\begin{array}{c}.35^{*} \\
(0.10,0.58)\end{array}$ & $\begin{array}{c}.44 * * \\
(0.14,0.68)\end{array}$ & $\begin{array}{c}.33 \\
(-0.02,0.61)\end{array}$ & $\begin{array}{c}.41^{*} \\
(0.13,0.63)\end{array}$ \\
\hline
\end{tabular}

*Correlation is significant at the 0.05 level (2-tailed). ** Correlation is significant at the 0.01 level (2-tailed)

Note: Confidence Intervals: 95\% Bias Corrected and Accelerated. Number of samples: 1000 Bootstrap Samples 


\section{Table 4.13}

Multiple Regression Analysis for the Visual-spatial Tasks: Predicting Children's SRL Skills and Performance from Maternal Support (T1).

Independent variables

\begin{tabular}{|c|c|c|c|c|c|c|c|c|c|c|c|c|c|c|c|c|c|c|c|c|c|c|c|c|}
\hline & \multirow[b]{2}{*}{$\mathrm{R}^{2}$} & \multirow[b]{2}{*}{$\begin{array}{l}\text { Adj. } \\
\mathrm{R}^{2}\end{array}$} & \multirow[b]{2}{*}{$\mathrm{F}$} & \multirow[b]{2}{*}{$\mathrm{p}$} & \multicolumn{5}{|c|}{ Maternal Contingency } & \multicolumn{5}{|c|}{ Autonomy support } & \multicolumn{5}{|c|}{ Children's age } & \multicolumn{5}{|c|}{ Maternal education } \\
\hline & & & & & $B$ & $\begin{array}{c}S E \\
B\end{array}$ & $\beta$ & $t$ & $p$ & $B$ & $S E B$ & $\beta$ & $t$ & $p$ & $B$ & $\begin{array}{c}S E \\
B\end{array}$ & $\beta$ & $t$ & $p$ & $B$ & $S E B$ & $\beta$ & $t$ & $p$ \\
\hline \multicolumn{25}{|l|}{$\begin{array}{l}\text { Outcome } \\
\text { variables }\end{array}$} \\
\hline $\begin{array}{l}\text { Cognitive } \\
\& \text { Metaco- } \\
\text { gnitive } \\
\text { skills }\end{array}$ & .577 & .536 & 14.113 & $<.001$ & $\begin{array}{l}.418 \\
(.043 \\
.884)\end{array}$ & .207 & $.328^{*}$ & 2.158 & $<.05$ & $\begin{array}{l}.297 \\
(-.087 \\
.842)\end{array}$ & .210 & .244 & 1.422 & $>.05$ & $\begin{array}{c}.047 \\
(.008, \\
.079)\end{array}$ & .023 & $.334^{*}$ & 2.268 & $<.05$ & - & - & - & - & - \\
\hline $\begin{array}{c}\text { Emotional \& } \\
\text { Motivational } \\
\text { skills }\end{array}$ & .443 & .389 & 8.205 & $<.001$ & - & - & - & - & - & $\begin{array}{l}.328 \\
\mathbf{( . 0 5 3} \\
.651)\end{array}$ & .166 & $.362^{*}$ & 2.147 & $\begin{array}{c}< \\
.05\end{array}$ & $\begin{array}{c}.032 \\
(-.020 \\
.073)\end{array}$ & .021 & .307 & 1.756 & $>.05$ & $\begin{array}{c}.106 \\
(-.114 \\
.280)\end{array}$ & .111 & .145 & 1.007 & $>.05$ \\
\hline $\begin{array}{l}\text { Autonomy } \\
\text { skills }\end{array}$ & .307 & .240 & 4.581 & $<.01$ & - & - & - & - & - & $\begin{array}{c}.323 \\
\mathbf{( . 1 3 5}, \\
. \mathbf{5 5 8})\end{array}$ & .101 & $.395^{*}$ & 2.098 & $\begin{array}{c}< \\
.05\end{array}$ & $\begin{array}{l}.000 \\
(- \\
.043 \\
.032)\end{array}$ & .019 & .003 & -.015 & $>.05$ & $\begin{array}{c}.199 \\
(-.026 \\
.394)\end{array}$ & .102 & .301 & 1.870 & $>.05$ \\
\hline Performance & .410 & .353 & 7.172 & $<.01$ & $\begin{array}{c}3.381 \\
(-1.569, \\
9.939)\end{array}$ & $\begin{array}{c}2.85 \\
0\end{array}$ & .208 & 1.155 & $>.05$ & $\begin{array}{c}3.482 \\
(-2.579, \\
10.263)\end{array}$ & 2.928 & $\begin{array}{l}.2 \\
24\end{array}$ & 1.105 & $>.05$ & $\begin{array}{c}.584 \\
(-.031 \\
1.078)\end{array}$ & .310 & .328 & 1.884 & $>.05$ & - & - & - & - & - \\
\hline
\end{tabular}

$* p<.05$ 
Table 4.14

Multiple Regression Analysis for the Language Task: Predicting Children's SRL Skills and Performance from Maternal Support (T1).

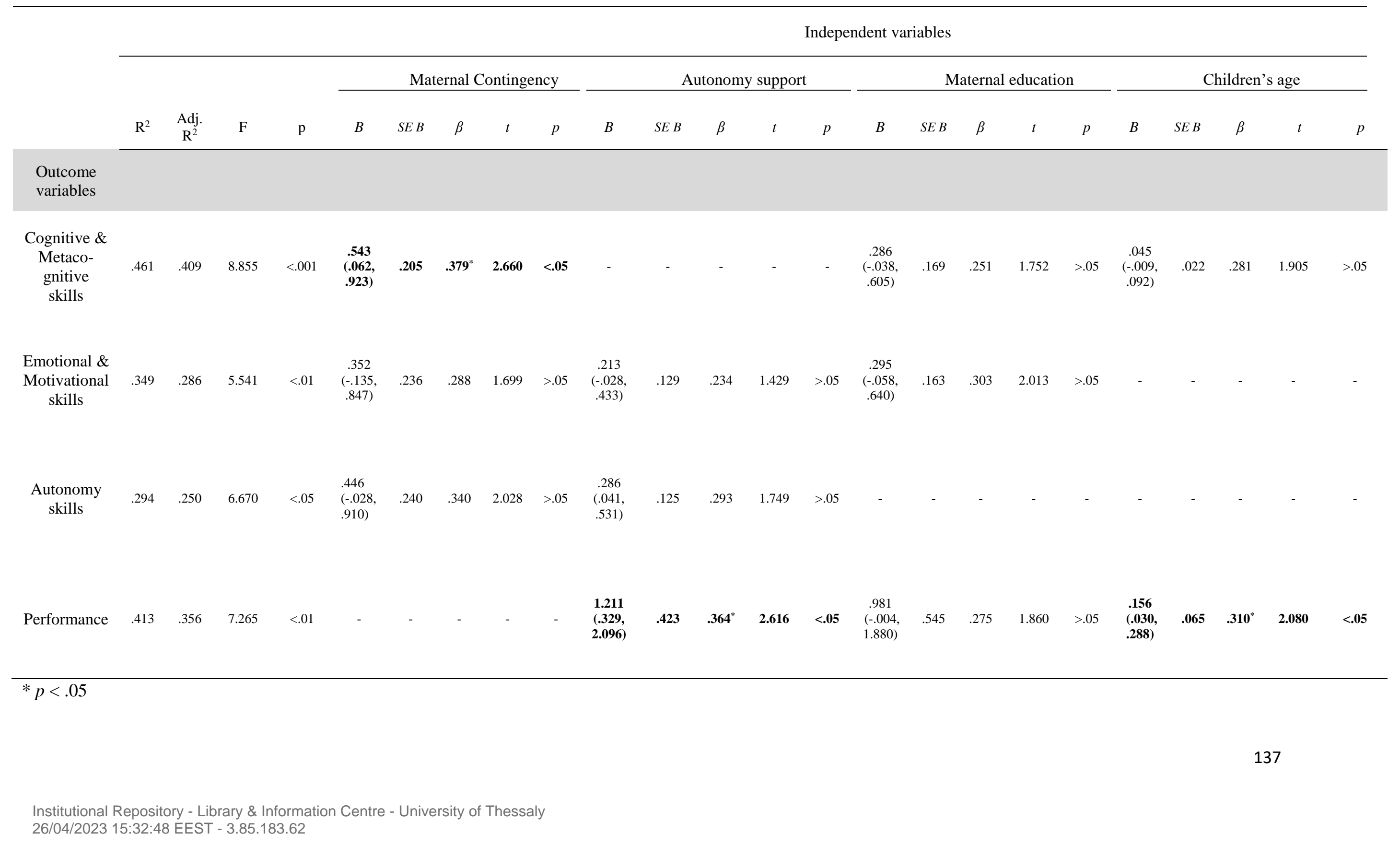




\subsubsection{Children's SRL skills and performance (Time 1)}

To examine whether and which of the children's SRL skills could predict children's performance, partial correlations were first performed controlling for children's age and then multiple regression analysis was run.

Table 4.15 depicts the correlations between children's SRL skills and performance for the Visual-spatial and Language tasks at Time 1. As expected, the results highlighted the significant positive correlations between these factors. Children's Cognitive and Metacognitive skills had the strongest correlation with children's performance, followed by children's Emotional and Motivational skills and by children's Autonomy skills (for the Visual-spatial tasks: $r=.60, p<.001 ; r=.47, p<.01 ; r=.40, p<.05$ respectively. For the Language tasks: $r=.94, p<.001 ; r=.77, p<.001 ; r=.70, p<.001$ respectively).

Based on the correlations found above, two multiple regression analyses were employed (for each one of the types of tasks) using the Bootstrap method, with children's Cognitive and Metacognitive skills, Emotional and Motivational skills and children's age as independent variables in the Visual-spatial tasks and with children's Cognitive and Metacognitive skills, Emotional and Motivational skills and Autonomy skills as independent variables in the Language tasks. Children's performance was the outcome variable. The bootstrap method was used reporting the $95 \%$ bias-corrected and accelerated confidence intervals for B (BCa CI), based on 1000 samples. The results, as shown in the table 4.16 are the same for both types of tasks. Children's Cognitive and Metacognitive skills independently predicted children's performance. For the Visual-spatial tasks, the model was able to predict $55.8 \%$ of the variance in children's performance $[F(3,31)=13.040, p<.001]$. For the Language task, the model was able to predict $91.6 \%$ of the variance in children's performance $[F(3,31)=112.627, p<.001]$. There were very strong effect sizes for the two regression outcomes $\left(f^{2}=1.06, f^{2}=9.8\right.$ respectively).

Collinearity statistics were run to check for possible problems with multicollinearity. The lowest Tolerance level was .263, and the highest variance inflation factor (VIF) was 3.797 for the first regression. The lowest Tolerance level was .224, and the highest variance inflation factor (VIF) was 4.474 for the second regression. 
Table 4.15

Partial Correlations between Children'SRL Skills and Performance Controlling for Children's Age (Time 1)

\begin{tabular}{|c|c|c|}
\hline & \multicolumn{2}{|c|}{ Cognitive Performance } \\
\hline & Visual-spatial tasks & Language tasks \\
\hline \multicolumn{3}{|l|}{ Children's skills } \\
\hline Cognitive and Metacognitive skills & $\begin{array}{c}.60 * * * \\
(0.38,0.77)\end{array}$ & $\begin{array}{c}.94 * * * \\
(0.90,0.96)\end{array}$ \\
\hline Emotional and Motivational skills & $\begin{array}{c}.47 * * \\
(0.17,0.70)\end{array}$ & $\begin{array}{c}.77 * * * \\
(0.57,0.90)\end{array}$ \\
\hline Autonomy skills & $\begin{array}{c}.40 * \\
(0.13,0.60)\end{array}$ & $\begin{array}{c}.70 * * * \\
(0.51,0.85)\end{array}$ \\
\hline
\end{tabular}

$* p<.05, * * p<.01, * * * p<.001$

Table 4.16

Multiple Regression Analysis for the Visual-spatial and Language Tasks: Predicting Children's Performance from Children's SRL Skills (T1)

Independent variables

\begin{tabular}{|c|c|c|c|c|c|c|c|c|c|c|c|c|c|c|c|c|c|c|c|c|c|c|c|c|}
\hline & \multirow[b]{2}{*}{$\mathrm{R}^{2}$} & \multirow[b]{2}{*}{$\begin{array}{l}\text { Adj. } \\
\mathrm{R}^{2}\end{array}$} & \multirow[b]{2}{*}{$\mathrm{F}$} & \multirow[b]{2}{*}{$\mathrm{p}$} & \multirow[b]{2}{*}{$B$} & \multicolumn{4}{|c|}{$\begin{array}{c}\text { Cognitive and Metacognitive } \\
\text { skills }\end{array}$} & \multirow[b]{2}{*}{$B$} & \multicolumn{4}{|c|}{$\begin{array}{l}\text { Emotional and Motivational } \\
\text { skills }\end{array}$} & \multicolumn{5}{|c|}{$\begin{array}{l}\text { Autonomy } \\
\text { skills }\end{array}$} & \multicolumn{5}{|c|}{ Age } \\
\hline & & & & & & $S E B$ & $\beta$ & $t$ & $p$ & & $S E B$ & $\beta$ & $t$ & $p$ & $B$ & $S E B$ & $\beta$ & $t$ & $p$ & $B$ & $S E B$ & $\beta$ & $t$ & $p$ \\
\hline \multicolumn{25}{|l|}{$\begin{array}{l}\text { Outcome } \\
\text { variables }\end{array}$} \\
\hline $\begin{array}{l}\text { Performa- } \\
\text { nce } \\
\text { (Visual- } \\
\text { spatial ) }\end{array}$ & .558 & .515 & 13.040 & $<.001$ & $\begin{array}{l}7.809 \\
(\mathbf{2 . 0 6 6} \\
\mathbf{1 5 . 7 6 5 )}\end{array}$ & 3.155 & $.611 *$ & 2.626 & $<.05$ & $\begin{array}{c}.637 \\
(-8.637 \\
8.092)\end{array}$ & 4.187 & .037 & .166 & $>.05$ & - & - & - & - & - & $\begin{array}{l}.269 \\
(-.307 \\
.666)\end{array}$ & .295 & .151 & .976 & $>.05$ \\
\hline $\begin{array}{l}\text { Performa- } \\
\quad \text { nce } \\
\text { (Language) }\end{array}$ & .916 & .908 & 112.627 & $<.001$ & $\begin{array}{l}2.604 \\
(2.124, \\
3.114)\end{array}$ & .241 & $.833^{*}$ & 9.994 & $<.01$ & $\begin{array}{l}.346 \\
(-.282 \\
1.209)\end{array}$ & .339 & .094 & .856 & $>.05$ & $\begin{array}{c}233 \\
(-.431 \\
.763)\end{array}$ & .301 & .068 & .732 & $>.05$ & - & - & - & - & - \\
\hline
\end{tabular}

$* p<.05$ 


\subsubsection{Mediation effects (Time 1)}

Following the above results, two mediation analyses were undertaken to understand better the role of both children's age and maternal supportive and contingent behaviours in predicting children's SRL skills and performance. Previous statistical analyses indicated that, in the Visual-spatial tasks, children's age was significantly associated with maternal Contingency, maternal Autonomy support, children's Cognitive and Metacognitive skills, Emotional and Motivational skills and performance. In addition, maternal Contingency was significantly associated with children's Cognitive and Metacognitive skills while maternal Autonomy support was significantly associated with all of the children's SRL skills. Hence, the first mediation analysis explored the relations between children's age, maternal Contingency, maternal Autonomy support and children's Cognitive and Metacognitive skills. The second mediation analysis investigated the relations between children's age, maternal Autonomy support and children's Emotional and Motivational skills.

For the Language tasks, children's age was significantly correlated with maternal Contingency and children's Cognitive and Metacognitive skills and performance. Moreover, maternal Contingency was also significantly correlated with children's Cognitive and Metacognitive skills and performance. Therefore, the first mediation analysis in the Language task investigated the relations between children's age, maternal Contingency and children's Cognitive and Metacognitive skills and the second mediation analysis explored the relations between children's age, maternal Contingency and children's performance. Furthermore, as maternal Autonomy support and children's Cognitive and Metacognitive skills were significantly related to children's performance in the Language task a third mediation analysis in the Language task was planned.

For this analysis, the PROCESS v3.0 tool for SPSS was used (Hayes, 2018) with 1000 Bootstrap Samples and 95\% Bias Corrected and Accelerated Confidence Intervals (BCa $\mathrm{CI})$. The results in the figures below include the regression coefficient for a path (independent variable predicting the mediator), the regression coefficient for $b$ path (predicting the dependent variable from the mediator), the regression coefficient for the c'path or direct path (independent variable predicting dependent variable with the mediator in the model) and the regression coefficient of the total effect or $\mathrm{c}$ path (independent variable predicting dependent variable without the mediator). If the $\mathrm{c}^{\prime}$ path is smaller than the $\mathrm{c}$ path $\left(0<\mathrm{c}^{\prime}<\mathrm{c}\right)$ then partial mediation occurs. 
For the Visual-spatial tasks, figure 1 shows that there was a significant indirect effect of children's age on children's Cognitive and Metacognitive skills through maternal Contingency (but not through autonomy support), $b=0.02$, BCaCI $(0.0015,0.0578), a b_{c s}=$ $0.15, \mathrm{BCaCI}(0.0117,0.3997)$, where $\mathrm{b}$ and $\mathrm{ab}_{\mathrm{cs}}$ refer to the indexes of the unstandardized and completely standardized indirect effect respectively. Even though the effect of children's age on children's Cognitive and Metacognitive skills was reduced when maternal contingency was included in the model, it remained statistically significant; maternal Contingency partially mediated the relation between children's age and children's Cognitive and Metacognitive skills. In the same way, figure 2 indicates that there was a significant indirect effect of children's age on children's Emotional and Motivational skills through maternal Autonomy support, $b=0.02$, BCaCI $(0.0029,0.0537), a b_{c s}=0.22$, BCaCI $(0.031$, 0.51). Even though the effect ( $c^{\prime}$ path) of children's age on children's Emotional and Motivational skills was reduced when maternal Autonomy support was included in the model, the effect remained statistically significant; maternal Autonomy support partially mediated the relation between children's age and children's Emotional and Motivational skills.

For the Language task, figure 3 and figure 4 supported that maternal Contingency partially mediated the effect of children's age on Cognitive and Metacognitive skills, $b=$ $0.02, \mathrm{BCaCI}(0.0017,0.054), a b_{c s}=0.14, \mathrm{BCaCI}(0.012,0.31)$ and on children's performance $\mathrm{b}=0.06, \mathrm{BCaCI}(0.0023,0.162), \mathrm{b}_{\mathrm{cs}}=0.12, \mathrm{BCaCI}(0.004,0.29)$. Finally, figure 5 shows that children's Cognitive and Metacognitive skills mediated the effect of maternal Autonomy support on children's performance, $\mathrm{b}=1.15, \mathrm{BCaCI}(0.25,2.09), a b_{c s}=0.35, \mathrm{BCaCI}(0.08$, 0.57). Regarding this mediation, the effect of maternal Autonomy on children's performance became insignificant when children's Cognitive and Metacognitive skills were included in the model (though, this does not mean that there was a full mediation).

On the whole, the mediation analyses revealed that the effect of children's age on some of the children's skills (Cognitive-Metacognitive skills, Emotional-Motivational skills and performance in the Language task) was partially mediated by these two maternal factors, implying that maternal Contingency and Autonomy support accounted for some of the relation between children's age, SRL skills and performance. Children's performance in the Visual-spatial tasks was predicted independently by children's Cognitive and Metacognitive skills. In the Language tasks, maternal Autonomy support predicted children's Cognitive and Metacognitive skills which in turn predicted children's performance. 


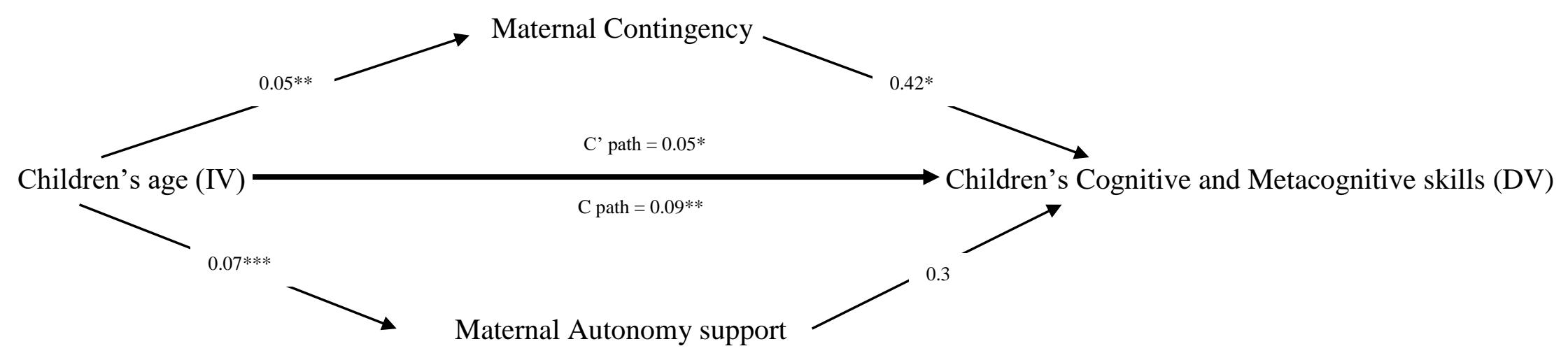

Figure 1. Parallel mediation model predicting children's Cognitive and Metacognitive skills from children's age, maternal Contingency and maternal Autonomy support in the Visual-spatial tasks T1

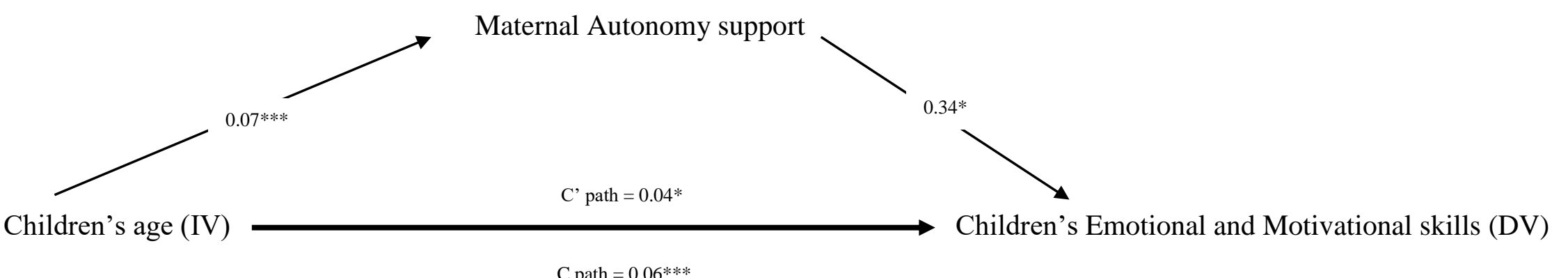

Figure 2. Mediation model predicting children's Emotional and Motivational skills from children's age and maternal Autonomy support in the Visual-spatial tasks T1 


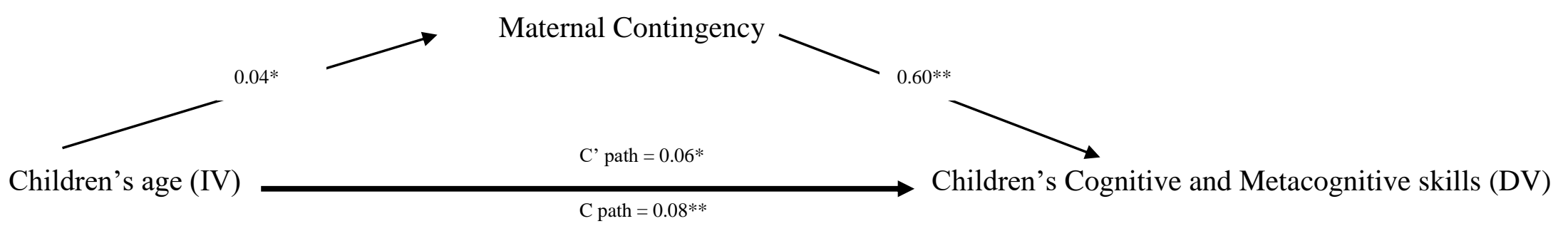

Figure 3. Mediation model predicting children's Cognitive and Metacognitive skills from children's age and maternal Contingency in the Language tasks T1

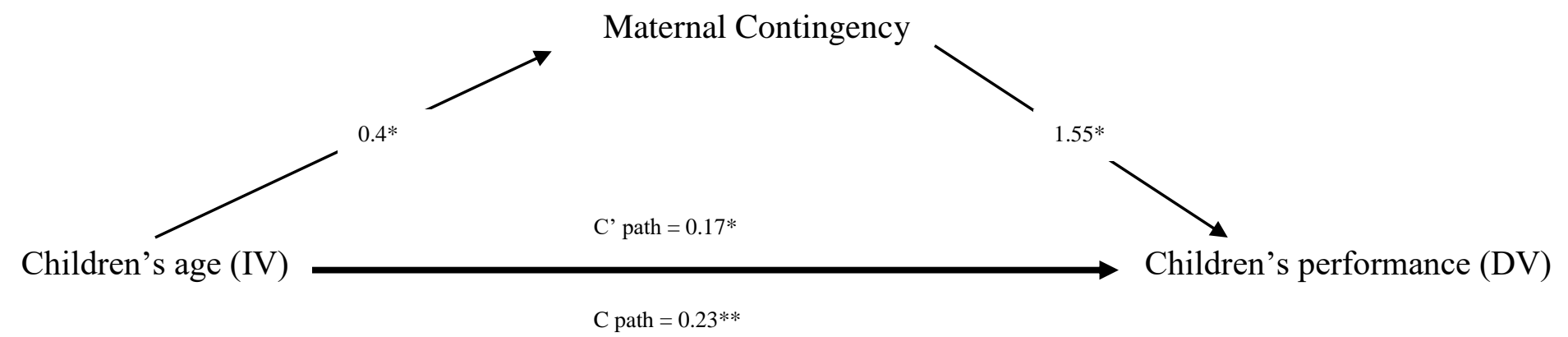

Figure 4. Mediation model predicting children's performance from children's age and maternal Contingency in the Language tasks T1 


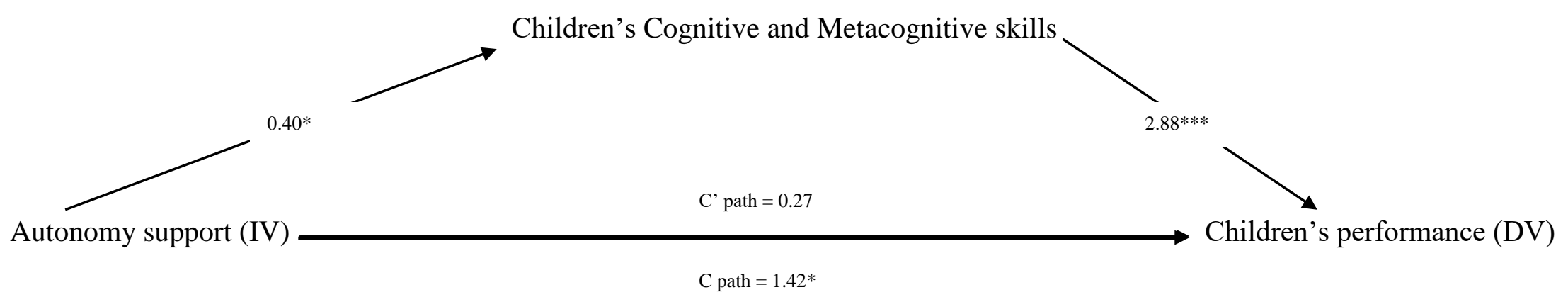

Figure 5. Mediation model predicting children's performance from maternal Autonomy support through children's Cognitive and Metacognitive skills in the Language tasks T1 


\subsection{Results from Time 2}

\subsubsection{Maternal supportive and contingent behaviours and children's SRL skills and performance (Time 2)}

The links between the different maternal dimensions and children's SRL skills and performance were additionally examined six months later (Time 2). To test the relations between maternal supportive and contingent behaviours (Time 2) and children's SRL skills and performance (Time 2), partial correlations were conducted controlling for children's age. Table 4.17 presents the correlations in the Visual-spatial and the Language tasks. What is interesting about the results in this table is that the results are similar to those in Time 1, but the correlations are stronger. In contrast to Time 1, maternal Contingency showed significant correlations with all of the children's SRL skills and performance in the Visual-spatial tasks, and maternal Emotional and Motivational support was significantly related to children's Motivational-Emotional and Autonomy skills but only in the Language task. Maternal Contingency and maternal Autonomy support were positively, significantly and strongly associated with almost all of the children's SRL skills and performance in both types of task.

To compare the significance of the difference in the correlations between Time 1 and 2, the Fisher r-to-z transformation was performed. The results showed that the correlations between children's Cognitive and Metacognitive skills and maternal Autonomy support in the Language tasks at Time 1 differed significantly from the corresponding at Time 2 . The correlation between these factors became significantly stronger after six months $(Z=-2.21, p$ $<.05)$. The rest of the studied correlations, although stronger at Time 2, were not significantly different from those at Time 1 .

Based on the correlations found above, four multiple regression analyses with the Enter Method and the use of the Bootstrap method were conducted with 1000 Bootstrap Samples and 95\% Bias Corrected and Accelerated Confidence Intervals for B (BCa CI). As mentioned in the previous section, the maximum number of the loaded predictors for each model was three due to the sample's restrictions. Therefore, the predictors were chosen following the higher correlations.

Table 4.18 reports the results from the multiple regressions performed in the Visualspatial tasks at Time 2. Three maternal predictors were loaded to the first two models (maternal Contingency, Autonomy support and children's age) and two predictors to the third and fourth model (maternal Contingency, Autonomy support). Overall, the four models were 
statistically significant. In particular, for children's Cognitive and Metacognitive skills, maternal Autonomy support and children's age were independent predictors. A significant model $F(3,31)=9.119, p<.001$, explained $46.9 \%$ of the outcome variable. However, the $p$ value of the confidence intervals for maternal Autonomy support was .092 and the biascorrected and accelerated confidence intervals for B contained zero, which yields concerns about the genuineness of the result. The $p$ value of the confidence intervals for children's age was .051, and the bias-corrected and accelerated confidence intervals for B did not contain zero. For children's Emotional and Motivational skills, maternal Autonomy support contributed independently to the model, $F(3,31)=9.715, p<.001$. However, the $p$ value for the confidence intervals of the maternal Autonomy support was .078 and the bias-corrected and accelerated confidence intervals for B contained zero, a finding that raises concerns about the genuineness of the result. In the third model, maternal Autonomy support and children's age were independent predictors of children's Autonomy skills. A significant model $[F(2,32)$ $=6.394, p<.01]$ predicted $28.6 \%$ of the variance in children's Autonomy behaviours. For children's performance, none of the predictors contributed independently to the model $[F(2$, $32)=11.357, p<.001]$. There were strong effect sizes for the first, second and fourth regression outcomes and a moderate effect size for the third regression outcome $\left(f^{2}=0.71, f^{2}\right.$ $=0.77, f^{2}=0.32, f^{2}=0.61$ respectively). Collinearity statistics showed that the lowest Tolerance value was .401 and the highest VIF value was 2.493 for the first and second regression model. For the third and fourth regression model, the lowest Tolerance value was .414 , and the highest VIF value was 2.416 . The collinearity statistics indicated no concerns with multicollinearity.

Table 4.19 presents the multiple regression analyses regarding the Language task. The predictors entered into the models were maternal Contingency, maternal Autonomy support and children's age. The four models were statistically significant in predicting children's SRL skills and performance. The results were almost consistent in the four models showing that maternal Autonomy support and children's age significantly contributed to each model. For children's Cognitive and Metacognitive skills, the model predicted $65.3 \%$ of its variance, and it was statistically significant $F(3,31)=19.421, p<.001$. For children's Emotional and Motivational skills, the model predicted $61.2 \%$ of its variance, $F(3,31)=16.324, p<.001$. The third model predicted $51.4 \%$ of the outcome variance $[F(3,31)=10.928, p<.001]$. Even though the bias-corrected and accelerated confidence intervals for B concerning children's age did not contain zero, the $p$ value for the confidence intervals was .055 which yields concerns about the genuineness of the result. Finally, in the last model, $54.9 \%$ of the variance 
was explained by the model $[F(3,31)=12.599, p<.001]$. There were strong effect sizes for all regression outcomes $\left(f^{2}=1.62, f^{2}=1.35, f^{2}=0.88, f^{2}=1.02\right.$ respectively). Collinearity statistics showed that the lowest Tolerance value was .749 and the highest VIF value was 1.334 for all models. No problem with multicollinearity was detected.

In general, the results of Time 2 of the study showed stronger and more complex correlations between maternal Contingency, maternal Autonomy support and children's SRL skills and performance in the Visual-spatial and Language tasks, in comparison to the correlations found at Time 1. Further statistical analyses (multiple regression) revealed that, in the Visual-spatial tasks, children's age and maternal Autonomy support were independent predictors of children's Cognitive and Metacognitive skills and maternal Autonomy support of children's Emotional-Motivational and Autonomy skills. Regarding the Language tasks, maternal Autonomy support and children's age consistently predicted almost all of the children's SRL skills and performance. 
Table 4.17

Partial Correlations between Maternal Support and Children's Skills and Performance in the Visual-spatial and Language Tasks (T2), Controlling for Children's Age

\begin{tabular}{|c|c|c|c|c|c|c|c|c|}
\hline & \multicolumn{6}{|c|}{ Children's SRL skills } & \multicolumn{2}{|c|}{ Performance } \\
\hline & \multicolumn{2}{|c|}{$\begin{array}{l}\text { Cognitive and Metacognitive } \\
\text { skills }\end{array}$} & \multicolumn{2}{|c|}{$\begin{array}{l}\text { Emotional and Motivational } \\
\text { skills }\end{array}$} & \multicolumn{2}{|c|}{$\begin{array}{l}\text { Autonomy } \\
\text { skills }\end{array}$} & \multirow[b]{2}{*}{$\begin{array}{c}\text { Visual-spatial } \\
\text { tasks } \\
\end{array}$} & \multirow[b]{2}{*}{$\begin{array}{l}\text { Language } \\
\text { tasks }\end{array}$} \\
\hline & $\begin{array}{c}\begin{array}{l}\text { Visual-spatial } \\
\text { tasks }\end{array} \\
\end{array}$ & $\begin{array}{l}\text { Language } \\
\text { tasks }\end{array}$ & $\begin{array}{l}\text { Visual-spatial } \\
\text { tasks } \\
\end{array}$ & $\begin{array}{c}\text { Language } \\
\text { tasks }\end{array}$ & $\begin{array}{c}\text { Visual-spatial } \\
\text { tasks } \\
\end{array}$ & $\begin{array}{c}\text { Language } \\
\text { tasks }\end{array}$ & & \\
\hline \multicolumn{9}{|l|}{ Maternal support } \\
\hline Maternal Contingency & $\begin{array}{c}.50 * * \\
(0.23,0.74)\end{array}$ & $\begin{array}{c}.24 \\
(-0.12,0.57)\end{array}$ & $\begin{array}{c}.54 * * \\
(0.25,0.76)\end{array}$ & $\begin{array}{c}.46^{* *} \\
(0.18,0.71)\end{array}$ & $\begin{array}{c}.36^{*} \\
(-0.04,0.66)\end{array}$ & $\begin{array}{c}.39^{*} \\
(0.01,0.69)\end{array}$ & $\begin{array}{c}.58 * * * \\
(0.37,0.76)\end{array}$ & $\begin{array}{c}.35^{*} \\
(-.03,0.64)\end{array}$ \\
\hline $\begin{array}{c}\text { Cognitive and } \\
\text { Metacognitive support }\end{array}$ & $\begin{array}{c}.20 \\
(-0.27,0.66)\end{array}$ & $\begin{array}{c}-.15 \\
(-0.44,0.22)\end{array}$ & $\begin{array}{c}.25 \\
(-0.21,0.70)\end{array}$ & $\begin{array}{c}-.14 \\
(-0.48 \\
0.22)\end{array}$ & $\begin{array}{c}.29 \\
(-0.09,0.74)\end{array}$ & $\begin{array}{c}-.11 \\
(-0.45,0.24)\end{array}$ & $\begin{array}{c}.16 \\
(-0.24,0.54)\end{array}$ & $\begin{array}{c}-.07 \\
(-0.38,0.25)\end{array}$ \\
\hline $\begin{array}{c}\text { Emotional and } \\
\text { Motivational support }\end{array}$ & $\begin{array}{c}.24 \\
(-0.10,0.55)\end{array}$ & $\begin{array}{c}.31 \\
(0.04,0.52)\end{array}$ & $\begin{array}{c}.26 \\
(-0.10,0.56)\end{array}$ & $\begin{array}{c}.44 * * \\
(0.11,0.71)\end{array}$ & $\begin{array}{c}.11 \\
(-0.32,0.54)\end{array}$ & $\begin{array}{c}.37^{*} \\
(0.10,0.59)\end{array}$ & $\begin{array}{c}.31 \\
(0.01,0.57)\end{array}$ & $\begin{array}{c}.21 \\
(-0.07,0.42)\end{array}$ \\
\hline Autonomy support & $\begin{array}{c}.58 * * * \\
(.0 .25,0.79)\end{array}$ & $\begin{array}{c}.73 * * * \\
(0.49,0.88) \\
\end{array}$ & $\begin{array}{c}.62 * * * \\
(0.40,0.77) \\
\end{array}$ & $\begin{array}{c}.66 * * * \\
(0,37,0.88) \\
\end{array}$ & $\begin{array}{c}.51 * * \\
(0.11,0.78) \\
\end{array}$ & $\begin{array}{c}.63 * * * \\
(0.38,0.81) \\
\end{array}$ & $\begin{array}{c}.57 * * * \\
(0.28,0.75) \\
\end{array}$ & $\begin{array}{c}.64 * * * \\
(0.38,0.85) \\
\end{array}$ \\
\hline
\end{tabular}

* Correlation is significant at the 0.05 level (2-tailed). **Correlation is significant at the 0.01 level (2-tailed). *** Correlation is significant at the 0.001 level.

Note: Confidence Intervals: 95\% Bias Corrected and Accelerated. Number of samples: 1000 Bootstrap Samples 
Table 4.18

Multiple Regression Analysis for the Visual-spatial Tasks: Predicting Children's SRL Skills and Performance from Maternal Support (T2).

Independent variables

\begin{tabular}{|c|c|c|c|c|c|c|c|c|c|c|c|c|c|c|c|c|c|c|}
\hline $\mathrm{R}^{2}$ & $\begin{array}{l}\text { Adj. } \\
\mathrm{R}^{2}\end{array}$ & $\mathrm{~F}$ & $\mathrm{p}$ & $B$ & $S E B$ & $\beta$ & $t$ & $p$ & $B$ & $S E B$ & $\beta$ & $t$ & $p$ & $B$ & $S E B$ & $\beta$ & $t$ & $p$ \\
\hline
\end{tabular}

Outcome

variables

Cognitive \&

Metacognitive

skills

.469

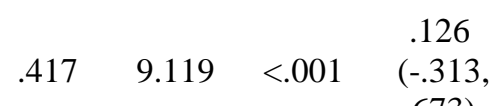

.262

.131

$.632>.05$

.376

(-.086,

$.213 .447 * 2.196<.05$

.032

.015

$.287 * 2.097$

$<.05$

Emotional \&

Motivational

$$
\text { skills }
$$

.485

$\begin{array}{cccc} & & & .134 \\ .435 \quad 9.715 & <.001 & (-.229\end{array}$

$.219 \quad .160 \quad .788 \quad>.05$

.339

(-.047,

.188

$.467 *$

$2.331<.05$

.023

$.731)$

$(-.004$,

.013

.233

.332

$(.045, \quad .139 \quad .574 * 2.470<.05$

Autonomy
skills

.286

$241-6.394<.01-035$

$\begin{array}{lllll}(-.276, & .143 & -.053 & -.227 & >.05\end{array}$

$.609)$

$\begin{array}{llllll}1.965 & 1.26 & & & & 1.379 \\ (-.685, & 1.26 & 379 & 1.804 & >05 & (-.736,\end{array}$

Performance

$\begin{array}{ccccc}.415 & .379 & 11.35 & <.001 & 1.965 \\ & & 7 & & (-.685 \\ & & & 4.734)\end{array}$
$3.502)$

$* p<.05$ 
Table 4.19

Multiple Regression Analysis for the Language Tasks: Predicting Children's SRL Skills and Performance from Maternal Support (T2)

Independent variables

\begin{tabular}{|c|c|c|c|c|c|c|c|c|c|c|c|c|c|c|}
\hline \multicolumn{5}{|c|}{ Maternal Contingency } & \multicolumn{5}{|c|}{ Autonomy support } & \multicolumn{5}{|c|}{ Children's age } \\
\hline$B$ & $S E B$ & $\beta$ & $t$ & $p$ & $B$ & $S E B$ & $\beta$ & $t$ & $p$ & $B$ & $S E B$ & $\beta$ & $t$ & $p$ \\
\hline
\end{tabular}

Outcome

variables

Cognitive \&

Metacognitive

skills

$\begin{array}{lllllllll}.653 & .619 & 19.421 & <.001 & \begin{array}{l}-.096 \\ (-.291, \\ .138)\end{array} & .104 & -.097 & -.794 & >.05\end{array}$

$\begin{aligned} .567 & \\ & 053\end{aligned}$

$\begin{array}{llllll}(.343, & .094 & .702 * & 5.822 & <.01 & \begin{array}{l}.019 \\ .724)\end{array} \\ & & & & & .085)\end{array}$

$\begin{array}{lll}.017 & .358 * & 3.252\end{array}$

$<.01$

\section{Emotional \&}

Motivational

$\begin{array}{lllll}.612 & .575 & 16.324 & <.001 & (-.005 \\ (185 & \end{array}$

.185

$.094 \quad .186 \quad 1.443 \quad>.05$

.404

$.404-085-498 \div-3.909$

.054

skills

$(-.005$,
$.346)$

$\begin{array}{lll}.186 & 1.443->.05\end{array}$

$.567)$

$.498 * \quad 3.909$

$\begin{array}{ll}<.01 \quad(.009 \\ & .093)\end{array}$

.093)

$.018 \quad .363 * 3.115<.05$

Autonomy

skills

$\begin{array}{lllll}.514 \quad .467 & 10.928 \quad<.001 & \begin{array}{l}.177 \\ (-.260 \\ .619)\end{array}\end{array}$

$\begin{array}{llll}.208 \quad .132 \quad .914 & >.05\end{array}$

.573
$(.276$
$.822)$

$\begin{array}{lll}.150 \quad .524 * & 3.671\end{array}$

$<.01$

.055

$\begin{array}{lllll}.001, & .027 & .278 * & 2.132 & <.05\end{array}$

.118)

\begin{tabular}{|c|c|c|c|c|c|c|c|c|c|c|c|c|c|c|c|c|c|c|c|}
\hline Performance & .549 & .506 & 12.599 & $<.001$ & $\begin{array}{c}.269 \\
(-.550 \\
1.334)\end{array}$ & .488 & .076 & .549 & $>.05$ & $\begin{array}{l}1.565 \\
(.805 \\
2.144)\end{array}$ & .337 & $.544 *$ & 3.962 & $<.01$ & $\begin{array}{c}.179 \\
(.044,\end{array}$ & .065 & $.342 *$ & 2.726 & $<.01$ \\
\hline
\end{tabular}

$* p<.05$ 


\subsubsection{Children's SRL skills and performance (Time 2)}

The next step of the statistical analysis was to explore whether and which of the children's SRL skills could predict children's performance. Once again, partial correlations controlling for children's age and multiple regressions were conducted with 1000 Bootstrap Samples and 95\% Bias Corrected and Accelerated Confidence Intervals for B(BCa CI).

Table 4.20 depicts the associations between children's SRL skills and performance for the Visual-spatial and Language tasks at Time 2 controlling for children's age. Similar to the results found at Time 1, children's Cognitive and Metacognitive skills, Emotional and Motivational skills and Autonomy skills were strongly associated with children's performance in the Visual-spatial and Language tasks (for the Visual-spatial tasks: $r=.88, p$ $<.001 ; r=.70, p<.001 ; r=.46, p<.01$ respectively. For the Language tasks: $r=.89, p<$. $001 ; r=.81, p<.001 ; r=.69, p<.001$ respectively).

To compare the significance of the difference in the correlations between Time 1 and Time 2, the Fisher r-to-z transformation was performed. The results showed that only the correlations between children's Cognitive and Metacognitive skills and performance in the Visual-spatial tasks at Time 1 differed significantly from the corresponding at Time 2 . The correlation between these factors became significantly stronger after six months $(Z=-2.73, p$ $<.01)$.

Based on the correlations found above, two multiple regression analyses were conducted using the Enter method and the Bootstrap method, with children's SRL skills as independent variables and children's performance as the outcome variable. The results, as at Time 1, underline the importance of children's Cognitive and Metacognitive skills in predicting children's performance in both types of tasks. Indeed, children's Cognitive and Metacognitive skills was the only independent predictor of children's performance, as shown in table 4.21. For the Visual-spatial task, the model was able to predict $78.6 \%$ of the variance in children's performance $[F(3,31)=37.881, p<.001]$. For the Language task, the model predicted $86.1 \%$ of the variance in children's performance $[F(3,31)=63.989, p<.001]$. There were very strong effect sizes for the two regression outcomes $\left(f^{2}=3.25, f^{2}=5.57\right.$ respectively). Collinearity statistics reported that the lowest Tolerance level was .223, and the highest variance inflation factor (VIF) was 4.488 for the first model. The lowest Tolerance level was .198, and the highest variance inflation factor (VIF) was 5.043 for the second model. 


\section{Table 4.20}

Partial Correlations between Children' SRL skills and Performance Controlling for Children's Age (T2)

\begin{tabular}{ccc}
\hline & \multicolumn{2}{c}{ Cognitive Performance } \\
\cline { 2 - 3 } Children's Skills & Visual-spatial tasks & Language tasks \\
\cline { 2 - 3 } Cognitive and Metacognitive skills & $.88^{* * *}$ & $.89^{* * * *}$ \\
Emotional and Motivational skills & $(0.77,0.95)$ & $(0.81,0.95)$ \\
Autonomy skills & $.70^{* * *}$ & $.81^{* * *}$ \\
& $(0.53,0.83)$ & $(0.68,0.89)$ \\
& $.46^{* *}$ & $.69 * * *$ \\
\end{tabular}

$* p<.05, * * p<.01, * * * p<.001$

Table 4.21

Multiple Regression Analysis for the Visual-spatial and Language Tasks: Predicting Children's Performance from Children's SRL Skills (T2)

Independent variables

\begin{tabular}{|c|c|c|c|c|c|c|c|c|c|c|c|c|c|c|c|c|c|c|}
\hline \multirow[b]{2}{*}{$\mathrm{R}^{2}$} & \multirow[b]{2}{*}{ Adj. $R^{2}$} & \multirow[b]{2}{*}{$\mathrm{F}$} & \multirow[b]{2}{*}{$\mathrm{p}$} & \multicolumn{5}{|c|}{$\begin{array}{l}\text { Cognitive and metacognitive } \\
\text { skills }\end{array}$} & \multicolumn{5}{|c|}{$\begin{array}{l}\text { Emotional and Motivational } \\
\text { skills }\end{array}$} & \multicolumn{4}{|c|}{$\begin{array}{l}\text { Autonomy } \\
\text { skills }\end{array}$} & \\
\hline & & & & $B$ & $S E B$ & $\beta$ & $t$ & $p$ & $B$ & $S E B$ & $\beta$ & $t$ & $p$ & $B$ & $S E B$ & $\beta$ & $t$ & $p$ \\
\hline
\end{tabular}

Outcome Variables

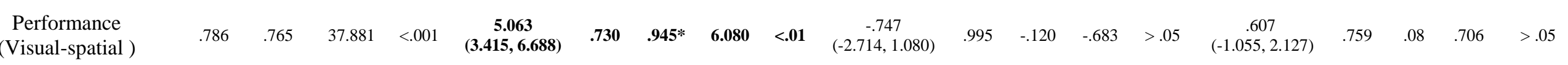

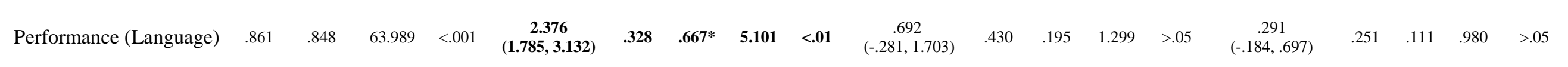




\subsubsection{Mediation effects (Time 2)}

Following the above results, two mediation analyses were undertaken for the Visualspatial tasks to clarify the role of both children's age and maternal supportive and contingent behaviours in predicting children's SRL skills and performance. Based on the correlations shown in the previous section, children's age was significantly associated with maternal Contingency, children's Cognitive and Metacognitive skills and children's Emotional and Motivational skills. In addition, maternal Contingency was also significantly associated with children's Cognitive and Metacognitive skills and Emotional and Motivational skills. However, the mediation analysis did not show that children's age significantly predicted maternal Contingency (a path). Therefore, the above mediation analyses were not completed.

Maternal Autonomy support and maternal Contingency were significantly related to children's Cognitive and Metacognitive skills and performance. It was also found that children's Cognitive and Metacognitive skills significantly predicted children's performance. Hence, the first mediation analysis explored the relations between maternal Autonomy support, children's Cognitive and Metacognitive skills and children's performance. The second mediation analysis investigated the relations between maternal Contingency, children's Cognitive and Metacognitive skills and children's performance.

Regarding the Language tasks, maternal Autonomy support was significantly correlated with children's Cognitive and Metacognitive skills and performance. It was also found that children's Cognitive and Metacognitive skills predicted children's performance. Thus, the mediation analysis in the Language task investigated the relations between maternal Autonomy support, children's Cognitive and Metacognitive skills and performance.

For the Visual-spatial tasks, figure 6 shows that there was a significant indirect effect of maternal Autonomy support on children's performance through children's Cognitive and Metacognitive skills, $b=2.29, \mathrm{BCaCI}(1.19,3.69), a b_{c s}=0.51, \mathrm{BCaCI}(0.28,0.75)$. The effect of maternal Autonomy support on performance became insignificant when children's Cognitive and Metacognitive skills were included in the model; children's Cognitive and Metacognitive skills mediated the relation between maternal Autonomy support and children's performance (this, however, does not support a full mediation). In the same way, figure 7 indicates that there was a significant indirect effect of maternal Contingency on children's performance through children's Cognitive and Metacognitive skills, $b=2.26$, $\mathrm{BCaCI}(1.11,3.9), a b_{c s}=0.44, \mathrm{BCaCI}(0.24,0.66)$. Again, the effect of maternal Contingency 
on performance became insignificant when children's Cognitive and Metacognitive skills were included in the model; children's Cognitive and Metacognitive skills mediated the relation between maternal Contingency and children's performance.

For the Language task, figure 8 supports that children's Cognitive and Metacognitive skills mediated the relation between maternal Autonomy support and children's performance, $\mathrm{b}=1.98, \mathrm{BCaCI}(1.38,2.68), a b_{c s}=0.69, \mathrm{BCaCI}(0.49,0.88)$. The effect of maternal Autonomy support on performance became insignificant when children's Cognitive and Metacognitive skills were included in the model.

Summarizing the results of Time 2, it was found that, as at Time 1, children's age, maternal Contingency and maternal Autonomy support were three factors that correlated significantly with children's SRL skills. In addition, the correlations noticed between these two maternal dimensions and children's SRL skills and performance were stronger at Time 2. Contrary to the results of Time 1 , mediation analysis did not show that these maternal dimensions were mediators in the relations between children's age and SRL skills. Children's Cognitive and Metacognitive skills were significant mediators in the relation between maternal Contingency, maternal Autonomy and children's performance for both types of tasks. 


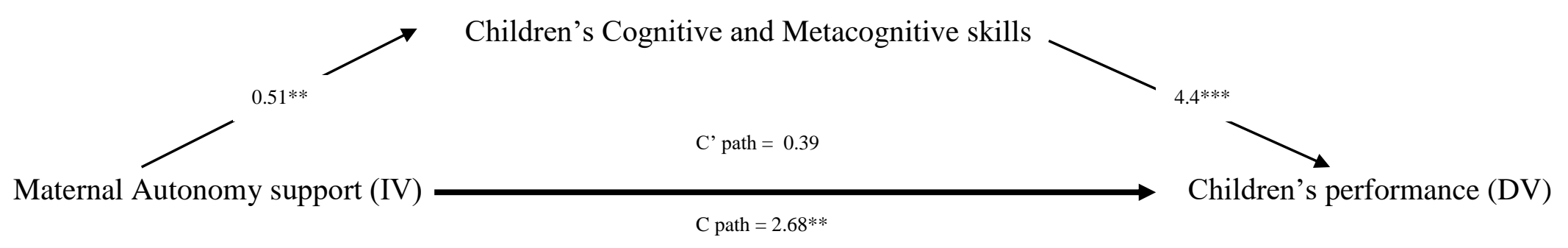

Figure 6. Mediation model predicting children's performance from maternal Autonomy support and children's Cognitive and Metacognitive skills in the Visual-spatial tasks T2

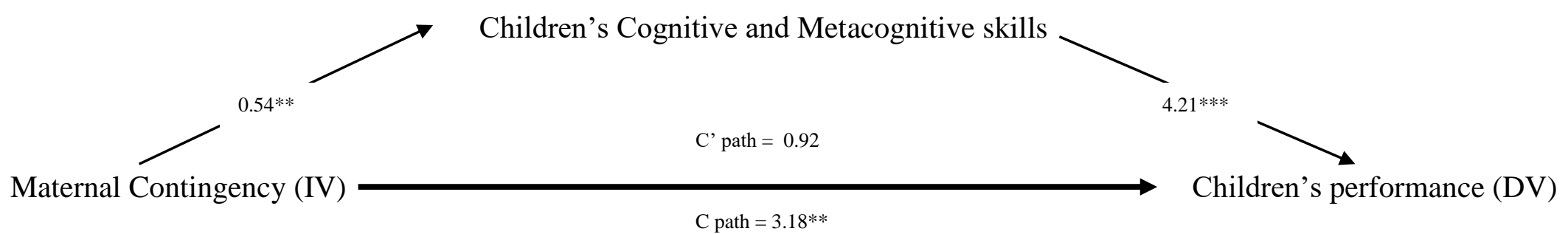

Figure 7. Mediation model predicting children's performance from maternal Contingency and children's Cognitive and Metacognitive skills in the Visual-spatial tasks T2 


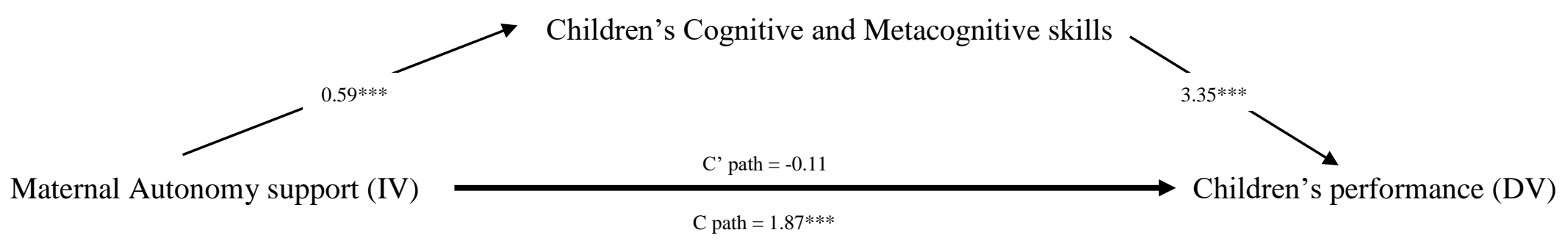

Figure 8. Mediation model predicting children's performance from maternal Autonomy support and children's Cognitive and Metacognitive skills in the Language task $\mathrm{T} 2$ 


\subsection{Longitudinal Findings: Results from Time 1 to Time 2}

\subsubsection{Early maternal supportive and contingent behaviours (T1) and children's later SRL skills and performance (T2)}

One of the main aims of this thesis was to study the longitudinal relations between maternal supportive and contingent behaviours and children's SRL skills and performance. To examine the above relations, the maternal dimensions from Time 1 and the children's SRL skills and performance from Time 2 were introduced in the analyses. Partial correlation, controlling for children's age at Time 2 and multiple regression analyses were employed. Table 4.22 demonstrates the correlations between maternal supportive and contingent behaviours at Time 1 and children's SRL skills and performance at Time 2.

Closer inspection of the table 4.22 reveals that statistically significant correlations were found, but only for the Visual-spatial tasks. Specifically, maternal Contingency (T1) was significantly and positively correlated with children's Cognitive-Metacognitive skills (T2) and performance (T2). Maternal Autonomy support (T1) was significantly and positively correlated with all of the children's SRL skills (T2) in the Visual-spatial tasks.

Given the correlations above, four multiple regression analyses were conducted with reference to the Visual-spatial domain, to investigate the predictive role of maternal Contingent (T1) and Autonomy support (T1) in children's SRL skills (T2) and performance (T2). Three predictors, that is, maternal Contingency, maternal Autonomy support and children's age were loaded into the first and last model using the Enter method. Two predictors were used for the second model (maternal Autonomy and children's age) and one predictor for the third model (Autonomy support). For these analyses, the bootstrap method was used reporting the $95 \%$ bias-corrected and accelerated confidence intervals for $\mathrm{B}$ in a parenthesis (BCa CI), based on 1000 samples.

Table 4.23 indicates that the four models significantly predicted children's SRL skills and performance in the Visual-spatial domain. Only one of the predictor variables, that is maternal Autonomy support (T1) significantly contributed to children's Emotional and Motivational skills (T2) and children's Autonomy skills (T2). Maternal Contingency (T1) was an independent predictor of children's performance (T2). For children's Cognitive and Metacognitive skills (T2), none of the predictor variables significantly and independently contributed to the model, which nevertheless was statistically significant, $F(3,31)=5.043, p$ $<.01$. For children's Emotional and Motivational skills (T2), the model explained $26.7 \%$ of 
the variance, and it was statistically significant, $F(2,32)=5.842, p<.01$. A significant model $[F(1,33)=6.197, p<.05]$ predicted $15.8 \%$ of children's Autonomy skills (T2). Finally, $28.9 \%$ of the variance in children's performance was explained by the model $[F(3,31)=$ 4.196, $p<.05]$. There were medium effect sizes for the first, second and last regression outcome $\left(f^{2}=0.36, f^{2}=0.28, f^{2}=0.28\right.$ respectively), and a small effect size for the third regression outcome $\left(f^{2}=0.15\right)$.

Collinearity statistics were run to check for possible problems with multicollinearity. The lowest Tolerance level was .462, and the highest variance inflation factor (VIF) was 2.165 for the first and last model. The lowest Tolerance level was .635 , and the highest variance inflation factor (VIF) was 1.575 for the second model, suggesting that there was no concern for multicollinearity issues.

Since the correlations between maternal supportive and contingent behaviours (T1) and children's SRL skills and performance (T2) were under .30 in the Language task, there was no suitable predictor for running a multiple regression analysis for this type of task. 
Table 4.22

Partial Correlations between Maternal Support (T1) and Children's Skills and Performance in the Visual-spatial and Language Tasks (T2), Controlling for Children's Age

Children's SRL skills (T2)

Performance (T2)

\begin{tabular}{|c|c|c|c|c|c|c|c|}
\hline \multicolumn{2}{|c|}{$\begin{array}{c}\text { Cognitive and Metacognitive } \\
\text { skills }\end{array}$} & \multicolumn{2}{|c|}{$\begin{array}{l}\text { Emotional and Motivational } \\
\text { skills }\end{array}$} & \multicolumn{2}{|c|}{$\begin{array}{l}\text { Autonomy } \\
\text { skills }\end{array}$} & \multirow[b]{2}{*}{$\begin{array}{l}\text { Visual-spatial } \\
\text { tasks }\end{array}$} & \multirow[b]{2}{*}{$\begin{array}{l}\text { Language } \\
\text { tasks }\end{array}$} \\
\hline $\begin{array}{l}\text { Visual-spatial } \\
\text { tasks }\end{array}$ & $\begin{array}{l}\text { Language } \\
\text { tasks }\end{array}$ & $\begin{array}{l}\text { Visual-spatial } \\
\text { tasks }\end{array}$ & $\begin{array}{l}\text { Language } \\
\text { tasks }\end{array}$ & $\begin{array}{l}\text { Visual-spatial } \\
\text { tasks }\end{array}$ & $\begin{array}{l}\text { Language } \\
\text { tasks }\end{array}$ & & \\
\hline
\end{tabular}

Maternal support (T1)

\begin{tabular}{|c|c|c|c|c|c|c|c|c|}
\hline Maternal Contingency & $\begin{array}{c}.38 * \\
(0.09,0.64)\end{array}$ & $\begin{array}{c}.04 \\
(-0.29,0.36)\end{array}$ & $\begin{array}{c}.27 \\
(-0.05,0.56)\end{array}$ & $\begin{array}{c}-.01 \\
(-0.35,0.26)\end{array}$ & $\begin{array}{c}-.02 \\
(-0.34,0.33)\end{array}$ & $\begin{array}{c}-.05 \\
(-0.38,0.23)\end{array}$ & $\begin{array}{c}.47 * * \\
(0.21,0.7)\end{array}$ & $\begin{array}{c}-.01 \\
(-0.34,0.29)\end{array}$ \\
\hline $\begin{array}{l}\text { Cognitive and } \\
\text { Metacognitive support }\end{array}$ & $\begin{array}{c}.02 \\
(-0.40,0.49)\end{array}$ & $\begin{array}{c}-.18 \\
(-0.49,0.13)\end{array}$ & $\begin{array}{c}.01 \\
(-0.36,0.45)\end{array}$ & $\begin{array}{c}-.06 \\
(-0.42,0.26)\end{array}$ & $\begin{array}{c}-.01 \\
(-0.36,0.46)\end{array}$ & $\begin{array}{c}-.15 \\
(-0.47,0.23)\end{array}$ & $\begin{array}{c}.02 \\
(-0.35,0.41)\end{array}$ & $\begin{array}{c}-.08 \\
(-0.42,0.25)\end{array}$ \\
\hline $\begin{array}{l}\text { Emotional and } \\
\text { Motivational support }\end{array}$ & $\begin{array}{c}.001 \\
(-0.38,0.40)\end{array}$ & $\begin{array}{c}-.15 \\
(-0.48,0.17)\end{array}$ & $\begin{array}{c}.01 \\
(-0.37,0.41)\end{array}$ & $\begin{array}{c}-.12 \\
(-0.42,0.16)\end{array}$ & $\begin{array}{c}-.24 \\
(-0.56,0.17)\end{array}$ & $\begin{array}{c}-.22 \\
(-0.47,0.06)\end{array}$ & $\begin{array}{c}-.01 \\
(-0.38,0.42)\end{array}$ & $\begin{array}{c}-.16 \\
(-0.46,0.14)\end{array}$ \\
\hline Autonomy support & $\begin{array}{c}.35^{*} \\
(0.07,0.62)\end{array}$ & $\begin{array}{c}.25 \\
(-.10,0.54)\end{array}$ & $\begin{array}{c}.37 * \\
(0.09,0.62)\end{array}$ & $\begin{array}{c}.18 \\
(-0.16,0.43)\end{array}$ & $\begin{array}{c}.37^{*} \\
(0.02,0.60)\end{array}$ & $\begin{array}{c}.08 \\
(-0.31,0.40)\end{array}$ & $\begin{array}{c}.33 \\
(0.01,0.63)\end{array}$ & $\begin{array}{c}.20 \\
(-0.10,0.48)\end{array}$ \\
\hline
\end{tabular}

*Correlation is significant at the 0.05 level (2-tailed). **Correlation is significant at the 0.01 level (2-tailed)

Note: Confidence Intervals: 95\% Bias Corrected and Accelerated. Number of samples: 1000 Bootstrap Samples 
Table 4.23

Multiple Regression Analysis for the Visual-spatial Tasks: Predicting Children's SRL Skills and Performance (T2) from Maternal Support (T1)

Independent Variables (T1)

\begin{tabular}{|c|c|c|c|c|c|c|c|c|c|c|c|c|c|c|c|c|c|c|c|}
\hline \multirow[b]{3}{*}{$\begin{array}{c}\text { Outcome } \\
\text { Variables (T2) }\end{array}$} & \multirow{3}{*}{$\mathrm{R}^{2}$} & \multirow{3}{*}{$\begin{array}{l}\text { Adj. } \\
\mathrm{R}^{2}\end{array}$} & \multirow{3}{*}{$\mathrm{F}$} & \multirow{3}{*}{$\mathrm{p}$} & \multicolumn{5}{|c|}{ Maternal Contingency } & \multicolumn{5}{|c|}{ Autonomy support } & \multicolumn{5}{|c|}{ Children's age (T2) } \\
\hline & & & & & $B$ & $S E B$ & $\beta$ & $t$ & $p$ & $B$ & $S E B$ & $\beta$ & $t$ & $p$ & $B$ & $S E B$ & $\beta$ & $t$ & $p$ \\
\hline & & & & & & & & & & & & & & & & & & & \\
\hline $\begin{array}{c}\text { Cognitive \& } \\
\text { Metaco- } \\
\text { gnitive skills }\end{array}$ & .328 & .263 & 5.043 & $<.01$ & $\begin{array}{c}.284 \\
(-.094 \\
.655)\end{array}$ & .198 & .276 & 1.439 & $>.05$ & $\begin{array}{l}.232 \\
(- \\
.106 \\
.722)\end{array}$ & .194 & .236 & 1.088 & $>.05$ & $\begin{array}{c}.019 \\
(-.026 \\
.062)\end{array}$ & .022 & .165 & .891 & $>.05$ \\
\hline $\begin{array}{c}\text { Emotional \& } \\
\text { Motivational } \\
\text { skills }\end{array}$ & .267 & .222 & 5.842 & $<.01$ & - & - & - & - & - & $\begin{array}{l}.366 \\
(.127, \\
.663)\end{array}$ & .143 & $.431 *$ & 2.272 & $<.05$ & $\begin{array}{c}.012 \\
(-.029 \\
.046)\end{array}$ & .019 & .126 & .662 & $>.05$ \\
\hline $\begin{array}{l}\text { Autonomy } \\
\text { skills }\end{array}$ & .158 & .133 & 6.197 & $<.05$ & - & - & - & - & - & $\begin{array}{l}.270 \\
(.082, \\
.448)\end{array}$ & .092 & $.398 *$ & 2.489 & $<.05$ & - & - & - & - & - \\
\hline Performance & .289 & .220 & 4.196 & $<.05$ & $\begin{array}{l}2.354 \\
(.254, \\
4.088)\end{array}$ & .991 & $.427 *$ & 2.166 & $<.05$ & $\begin{array}{c}.796 \\
(- \\
1.298\end{array}$ & 1.165 & .151 & .677 & $>.05$ & $\begin{array}{c}.002 \\
(-.260 \\
.228)\end{array}$ & .125 & .003 & .017 & $>.05$ \\
\hline
\end{tabular}

$* p<.05$ 


\subsubsection{Children's early SRL skills (T1) and children's later SRL skills and performance}

(T2)

To test whether children's early (T1) SRL skills would significantly predict children's later (T2) SRL skills and performance, partial correlations were first conducted controlling for children's age at Time 2.

As shown in table 4.24, there were some significant and positive correlations between children's early SRL skills and children's later SRL skills and performance but only for the Visual-spatial tasks. Specifically, children's early Cognitive and Metacognitive skills were significantly correlated to children's later Cognitive and Metacognitive skills $(r=.55, p<$ $.01)$, later Emotional and Motivational skills $(r=.43, p<.05)$ and later performance $(r=.56$, $p<.01)$. Children's early Emotional and Motivational skills were significantly correlated to children's later Cognitive and Metacognitive skills $(r=.36, p<.05)$, later Emotional and Motivational skills $(r=.34, p<.05)$, and later performance $(r=.35, p<.05)$. Finally, children's early Autonomy skills were significantly associated with children's later Cognitive and Metacognitive skills $(r=.47, p<.01)$ and later Emotional and Motivational skills $(r=$ $.46, p<.01)$. There was only one significant correlation in the Language tasks, concerning children's early Autonomy skills and children's later Cognitive and Metacognitive skills ( $r=$ $.35, p<.05)$.

Table 4.25 presents the multiple regression analyses conducted for the Visual-spatial tasks, to examine further the role of each one of the early SRL skills in predicting children's later SRL skills and performance. Since the correlations for children's Autonomy skills were weak (<.30), there were no predictors for this outcome variable. For the other two children's SRL skills and performance, three predictors were loaded into the model according to the correlations found above. For these analyses, the bootstrap method was used reporting the 95\% bias-corrected and accelerated confidence intervals for $\mathrm{B}$ in a parenthesis (BCa $\mathrm{CI}$ ), based on 1000 samples.

The results suggested that the three models were statistically significant for predicting children's later SRL skills and performance. However, only children's early Cognitive and Metacognitive skills was an independent predictor of children's later Cognitive and Metacognitive skills and performance. For children's later Cognitive and Metacognitive skills, the model explained $48.1 \%$ of the variance, which was found to significantly predict the outcome $F(3,31)=9.559, p<.001$. For children's Emotional and Motivational skills, the 
model was significant, $F(3,31)=6.467, p<.01$, but none of the predictor variables contributed independently to the model. For children's performance (T2), a significant model explained $38 \%$ of the outcome's variance, $F(3,31)=6.330, p<.01$. There were large effect sizes for the three regression outcomes $\left(\mathrm{f}^{2}=0.75, f^{2}=0.48\right.$ and $f^{2}=0.47$ respectively). Collinearity statistics showed that the lowest Tolerance level was .506, and the largest variance inflation factor (VIF) was 1.975 for the first and second regression model, and .272 and 3.679 respectively for the last regression model, which suggests no concerns for multicollinearity issues.

With reference to the Language task (table 4.26), two multiple regression analyses were conducted with three predictors (children's early Cognitive and Metacognitive skills, children's early Autonomy skills and children's age at Time 2) for the first model and two predictors (children's early Cognitive and Metacognitive skills and children's age at Time 2) for the last model. The outcome variables of the two regressions were children's later Cognitive and Metacognitive skills and children's later performance respectively. Even though the models were statistically significant, none of the predictors significantly and independently contributed to the model. For children's Cognitive and Metacognitive skills (T2), the model predicted $33.3 \%$ of the variance, $F(3,31)=5.151, p<.01$. For children's performance (T2), the model explained $26.1 \%$ of the variance, $F(2,32)=7.005, p<.05$. There were moderate effect sizes for the two regression outcomes $\left(f^{2}=0.37\right.$ and $f^{2}=0.35$ respectively). Collinearity statistics were run to check for possible problems with multicollinearity. The lowest Tolerance level was .397, and the largest variance inflation factor (VIF) was 2.516 for the first model and .725 and 1.378 for the last model, indicating no multicollinearity issues. 
Table 4.24

Partial Correlations between Children's SRL skills (Time 1) and Children's SRL skills and Performance (Time 2) Controlling for Children's Age

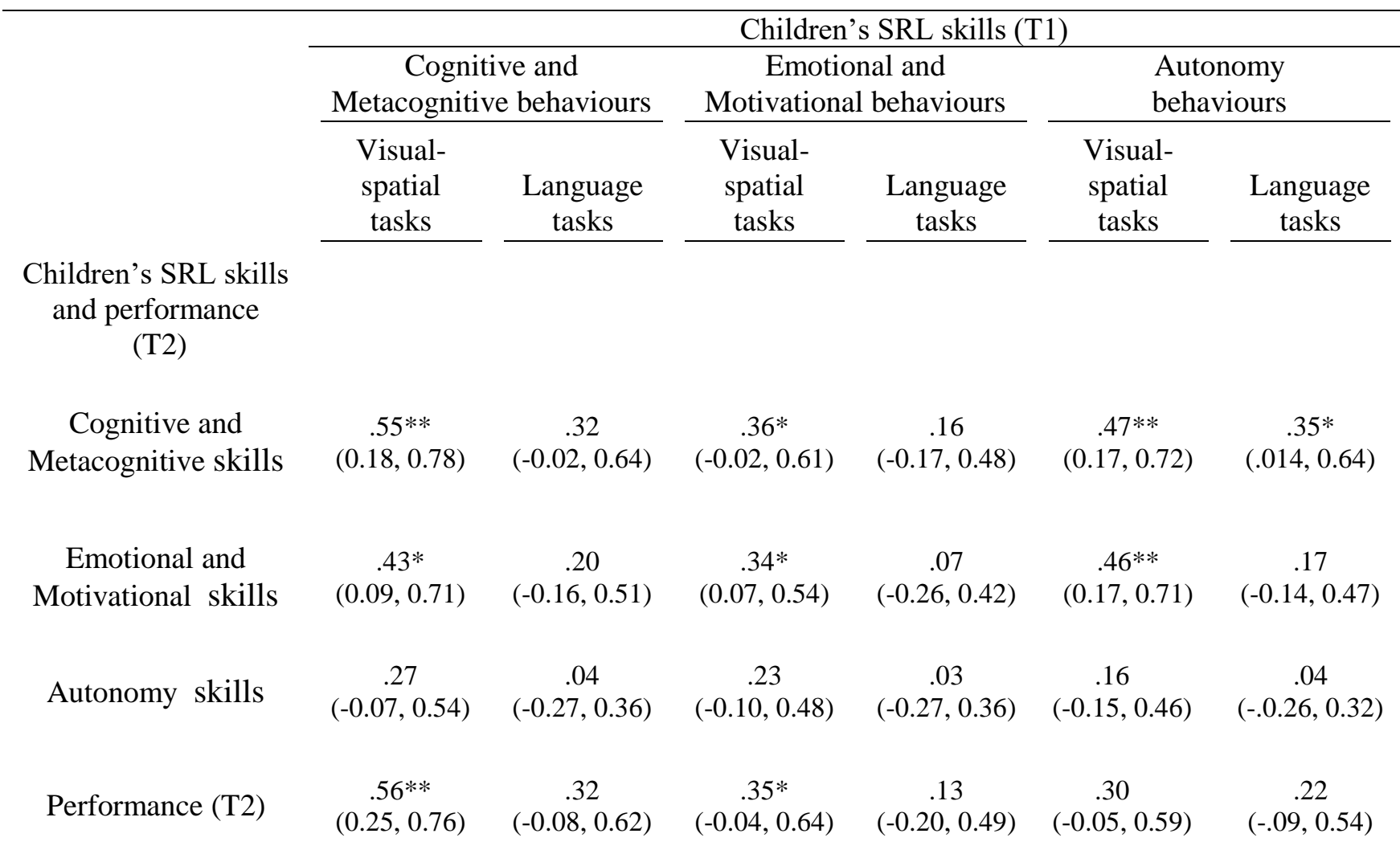

*Correlation is significant at the 0.05 level (2-tailed). **Correlation is significant at the 0.01 level (2-tailed)

Note: Confidence Intervals: 95\% Bias Corrected and Accelerated. Number of samples: 1000 Bootstrap

Samples 


\section{Table 4.25}

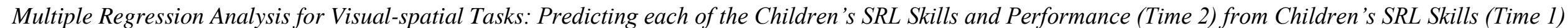

\begin{tabular}{|c|c|c|c|c|c|c|c|c|c|c|c|c|c|c|c|c|c|c|c|c|c|c|c|c|}
\hline & \multirow[b]{3}{*}{$R^{2}$} & \multicolumn{23}{|c|}{ Independent variables } \\
\hline & & \multirow[b]{2}{*}{$\begin{array}{r}\text { Adj } \\
R^{2}\end{array}$} & \multirow[b]{2}{*}{$F$} & \multirow[b]{2}{*}{$p$} & \multicolumn{5}{|c|}{$\begin{array}{c}\text { Cognitive and Metacognitive } \\
\text { skills (T1) }\end{array}$} & \multicolumn{5}{|c|}{$\begin{array}{c}\text { Emotional and Motivational } \\
\text { skills (T1) }\end{array}$} & \multicolumn{5}{|c|}{ Autonomy skills (T1) } & \multicolumn{5}{|c|}{ Age (T2) } \\
\hline & & & & & $B$ & $S E B$ & $\beta$ & $t$ & $p$ & $B$ & $S E B$ & $\beta$ & $t$ & $p$ & $B$ & $\begin{array}{l}S E \\
B\end{array}$ & $\beta$ & $t$ & $p$ & $B$ & $S E B$ & $\beta$ & $t$ & $p$ \\
\hline \multicolumn{25}{|l|}{$\begin{array}{l}\text { Outcome } \\
\text { Variables }\end{array}$} \\
\hline $\begin{array}{l}\text { Cognitive \& } \\
\text { Metacognitive } \\
\text { skills (T2) }\end{array}$ & .481 & .430 & 9.559 & $<.001$ & $\begin{array}{l}.386 \\
(.043, \\
.806)\end{array}$ & .186 & $.478^{*}$ & 2.629 & $<.05$ & - & - & - & - & - & $\begin{array}{c}.328 \\
(-.120 \\
.727)\end{array}$ & .220 & .273 & 1.749 & $>.05$ & $\begin{array}{r}.004 \\
(-.027 \\
.043)\end{array}$ & .019 & .040 & .245 & $>.05$ \\
\hline $\begin{array}{c}\text { Emotional \& } \\
\text { Motivational } \\
\text { skills (T2) }\end{array}$ & .385 & .325 & 6.467 & $<.01$ & $\begin{array}{c}.228 \\
(-.086 \\
.677)\end{array}$ & .178 & .327 & 1.650 & $>.05$ & - & - & - & - & - & $\begin{array}{c}.343 \\
(-.094 \\
.695)\end{array}$ & .216 & .330 & 1.948 & $>.05$ & $\begin{array}{c}.007 \\
(-.028 \\
.043)\end{array}$ & .017 & .070 & .394 & $>.05$ \\
\hline $\begin{array}{l}\text { Autonomy } \\
\text { skills (T2) }\end{array}$ & - & - & - & - & - & - & - & - & - & - & - & - & - & - & - & - & - & - & - & - & - & - & - & - \\
\hline $\begin{array}{l}\text { Performance } \\
\quad(\mathrm{T} 2)\end{array}$ & .380 & .320 & 6.330 & $<.01$ & $\begin{array}{l}3.637 \\
(.479 \\
6.324)\end{array}$ & 1.116 & $.841^{*}$ & 3.098 & $<.01$ & $\begin{array}{c}-1.259 \\
(-3.610 \\
1.786)\end{array}$ & 1.502 & -.216 & -.816 & $>.05$ & - & - & - & - & - & $\begin{array}{c}-.062 \\
(-.253 \\
.117)\end{array}$ & .107 & -.103 & -.573 & $>.05$ \\
\hline
\end{tabular}

$* p<.05$ 


\section{Table 4.26}

Multiple Regression Analysis for Language Tasks: Predicting each of the Children's SRL Skills and Performance (T2) from Children's SRL Skills (T1)

\begin{tabular}{|c|c|c|c|c|c|c|c|c|c|c|c|c|c|c|c|c|c|c|c|}
\hline & \multirow[b]{3}{*}{$R^{2}$} & \multirow[b]{3}{*}{$\underset{R^{2}}{A d j}$} & \multirow[b]{3}{*}{$F$} & \multirow[b]{3}{*}{$p$} & \multicolumn{15}{|c|}{ Independent variables } \\
\hline & & & & & \multicolumn{5}{|c|}{$\begin{array}{c}\text { Cognitive and metacognitive } \\
\text { behaviours (T1) }\end{array}$} & \multicolumn{5}{|c|}{$\begin{array}{c}\text { Autonomy } \\
\text { behaviours (T1) }\end{array}$} & \multicolumn{5}{|c|}{ Age } \\
\hline & & & & & $B$ & $S E B$ & $\beta$ & $t$ & $p$ & $B$ & $S E B$ & $\beta$ & $t$ & $p$ & $B$ & $S E B$ & $\beta$ & $t$ & $p$ \\
\hline \multicolumn{20}{|l|}{$\begin{array}{l}\text { Outcome } \\
\text { Variables }\end{array}$} \\
\hline $\begin{array}{l}\text { Cognitive \& } \\
\text { metacognitive } \\
\text { skills (T2) }\end{array}$ & .333 & .268 & 5.151 & $<.01$ & $\begin{array}{c}.141 \\
(-.379 \\
.573)\end{array}$ & .235 & .155 & .666 & $>.05$ & $\begin{array}{c}.222 \\
(-.184 \\
.644)\end{array}$ & .220 & .223 & 1.100 & $>.05$ & $\begin{array}{l}.051 \\
(-.001 \\
.114)\end{array}$ & .026 & .350 & 1.980 & $>.05$ \\
\hline $\begin{array}{c}\text { Emotional \& } \\
\text { motivational } \\
\text { skills (T2) }\end{array}$ & - & - & - & - & - & - & - & - & - & - & - & - & - & - & - & - & - & - & - \\
\hline $\begin{array}{l}\text { Autonomy skills } \\
\text { (T2) }\end{array}$ & - & - & - & - & - & - & - & - & - & - & - & - & - & - & - & - & - & - & - \\
\hline $\begin{array}{l}\text { Performance } \\
\text { (T2) }\end{array}$ & .305 & .261 & 7.005 & $<.05$ & $\begin{array}{l}1.070 \\
(-.105 \\
2.495)\end{array}$ & .655 & .330 & 1.905 & $>.05$ & - & - & - & - & - & $\begin{array}{l}.158 \\
(.012 \\
.314\end{array}$ & .084 & .302 & 1.747 & $>.05$ \\
\hline
\end{tabular}




\subsubsection{Mediation effects}

Following the above results, four mediation analyses were undertaken for the Visualspatial tasks to examine the relations between maternal supportive and contingent behaviours (T1), children's SRL skills (T2) and performance (T2). Based on the correlations above, maternal Autonomy support (T1) was significantly associated with children's Cognitive and Metacognitive skills at Time 2. Moreover, maternal Autonomy support (T1) was significantly associated with all of the children's skills (T1) which were also significantly related to children's Cognitive and Metacognitive skills (T2). Therefore, the first parallel mediation explored the relation between the above factors. The same mediation analysis, but with children's Emotional and Motivational skills (T2) as a dependent variable, was conducted. However, for this mediation, none of the children's SRL skills (T1) significantly predicted the dependent variable, and therefore these variables could not be considered as mediators in the above relation.

In addition, maternal Contingency (T1) was significantly associated with both children's Cognitive and Metacognitive skills (T1 and T2) and children's performance (T2). Taking into account that children's Cognitive and Metacognitive skills (T1 and T2) significantly predicted children's performance (T2), the second parallel mediation explored these relations. Finally, maternal Contingency (T1) was significantly related to children's Cognitive and Metacognitive skills (T1 and T2), and children's Cognitive and Metacognitive skills (T1) significantly predicted children's Cognitive and Metacognitive skills (T2). Therefore, the third mediation explored the relation between the above factors.

Figure 9 shows that there was a significant indirect effect of maternal Autonomy support (T1) on children's Cognitive and Metacognitive skills (T2) through children's Cognitive and Metacognitive skills (T1), $b=0.45, \mathrm{BCaCI}(0.07,0.86), a b_{c s}=0.46, \mathrm{BCaCI}$ $(0.08,0.82)$. Children's Emotional and Motivational skills (T1) did not significantly predict children's Cognitive and Metacognitive skills (T2) as shown in figure 9. Therefore, this variable did not act as a mediator. Even though, children's Autonomy skills seemed to act as a mediator (considering the $p$ values), the BCa CI of the indirect effect included zero. Thus, the result could not be considered as genuine, $b=0.17$, BCaCI $(-0.02,0.47)$. Children's Cognitive and Metacognitive skills (T1) partially mediated the relation between maternal Autonomy support (T1) and children's Cognitive and Metacognitive skills (T2). 
Figure 10 indicates that there was a significant indirect effect of maternal Contingency (T1) on children's performance (T2) through children's Cognitive and Metacognitive skills at Time 2 but not through children's Cognitive and Metacognitive skills at Time $1, b=2.31, \mathrm{BCaCI}(0.99,4.0), a b_{c s}=0.41, \mathrm{BCaCI}(0.20,0.68)$. Children's Cognitive and Metacognitive skills (T2) partially mediated the relation between maternal Contingency (T1) and children's performance (T2). Finally, figure 11 shows that there was a significant indirect effect of maternal Contingency (T1) on children's Cognitive and Metacognitive skills (T2) through children's Cognitive and Metacognitive skills (T1), $b=0.37, \mathrm{BCaCI}(0.14$, $0.72), a b_{c s}=0.36, \mathrm{BCaCI}(0.15,0.66)$.

One last mediation analysis was tested which was the result of the findings of the second and third mediation analysis. These two mediation analyses showed that the effect of maternal Contingency (T1) on children's Cognitive and Metacognitive skills (T2) was mediated by children's Cognitive and Metacognitive skills (T1) (Figure 11) and that the effect of maternal Contingency on children's performance (T2) was mediated by children's Cognitive and Metacognitive skills (T2) (Figure 10). Therefore, the serial mediation tested whether maternal Contingency (T1) positively predicted children's Cognitive and Metacognitive skills (T1) which in turn positively predicted children's Cognitive and Metacognitive skills (T2) which in turn positively predicted children's performance (T2). The results confirmed the above hypothesis and indicated that there was a significant indirect effect, $b=1.66$, BCaCI $(0.62,3.57), a b_{c s}=0.30$, BCaCI $(0.12,0.58)$.

Bringing together the findings from the short-term longitudinal study, it seems that significant paths emerged only with reference to the Visual-spatial domain. Maternal Contingency and maternal Autonomy at Time 1 were the main factors that were significantly correlated with almost all of the children's SRL skills and performance at Time 2. Children's SRL skills at Time 1 were also significantly linked to children's SRL skills and performance at Time 2 (except for Autonomy skills at Time 2). Further mediation analyses indicated that children's Cognitive and Metacognitive skills at Time 1 mediated the relations between maternal Contingency (Time 1) and children's Cognitive and Metacognitive skills at Time 2 as well as between maternal Autonomy support at Time 1 and children's Cognitive and Metacognitive skills at Time 2. In addition, it was found that children's Cognitive and Metacognitive skills at Time 1 and children's Cognitive and Metacognitive skills at Time 2 mediated the relations between maternal Contingency (T1) and children's performance at Time 2 in a serial mediation model. 


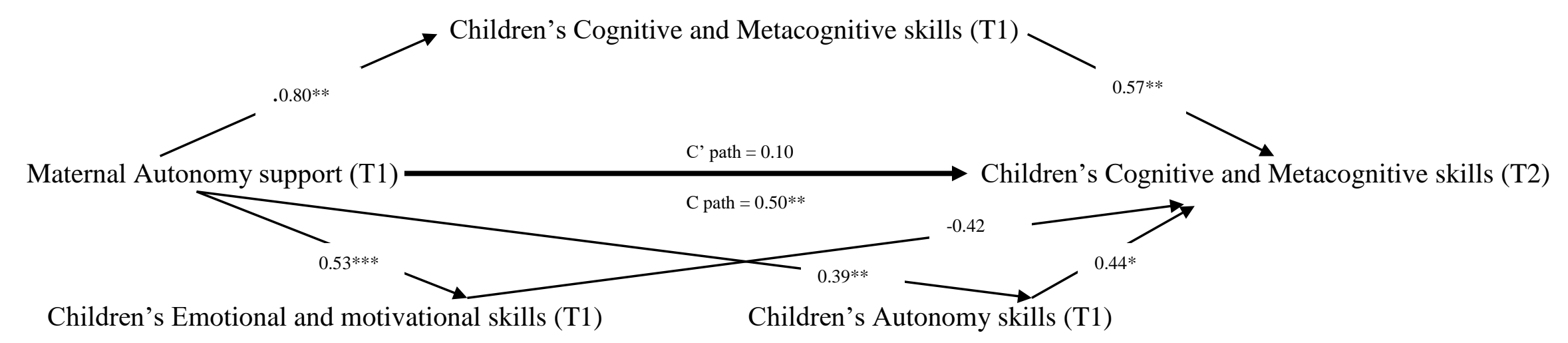

Figure 9. Parallel mediation model predicting children's Cognitive and Metacognitive skills (T2) from maternal Autonomy support (T1) and children's SRL skills (T1) in the Visual-spatial tasks

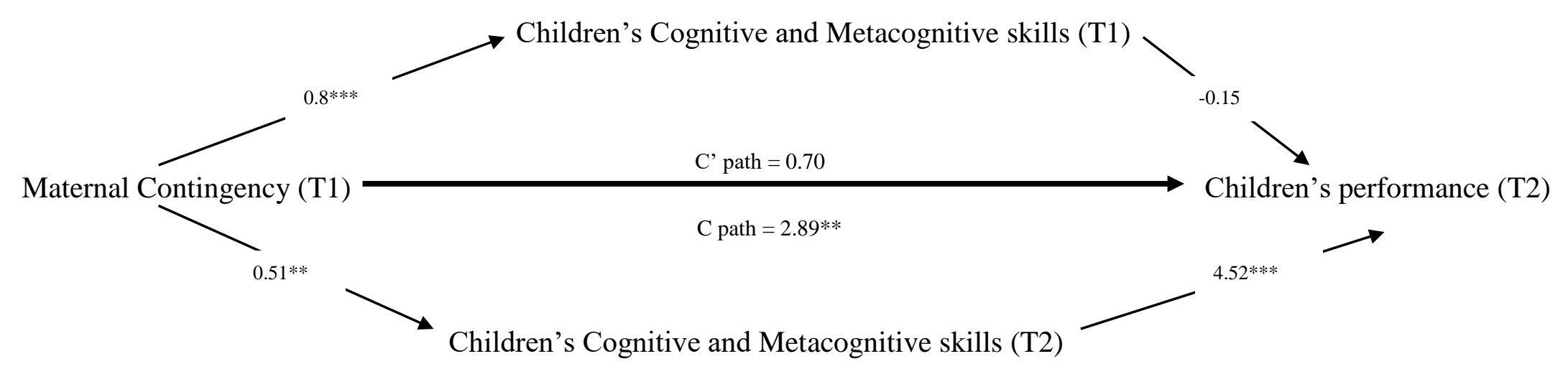

Figure 10. Parallel mediation model predicting children's performance (T2) from maternal Contingency (T1) and children's Cognitive and Metacognitive skills (T1 and T2) in the Visual-spatial tasks 
Children's Cognitive and Metacognitive skills (T1)

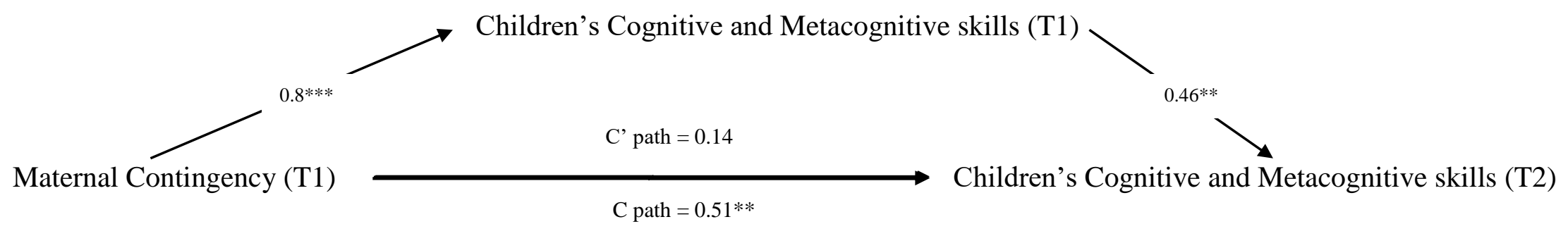

Figure 11. Mediation model predicting children's Cognitive and Metacognitive skills (T2) from maternal Contingency (T1) and children's Cognitive and Metacognitive skills (T1) in the Visual-spatial tasks

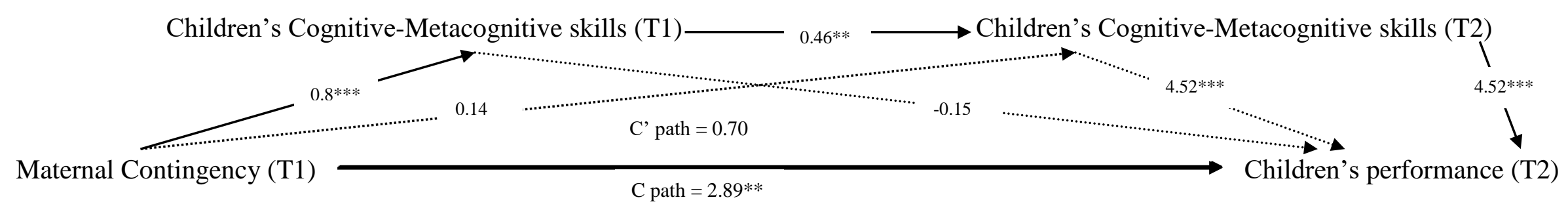

Figure 12. Serial mediation model predicting children's performance (T2) from maternal Contingency (T1) and children's Cognitive and Metacognitive skills (T1 and T2) in the Visual-spatial tasks 


\subsection{Longitudinal Observations in Maternal Supportive and Contingent Behaviours and in Children's SRL Skills}

Paired t-tests (for the parametric variables) and the Wilcoxon signed rank test (for non-parametric variables) were employed to determine whether there were statistically significant differences in maternal supportive and contingent behaviours and children's SRL skills over time.

Paired t-tests were implemented for maternal Cognitive and Metacognitive support (T1 and T2) and maternal Emotional and Motivational support (T1 and T2) for both cognitive domains. The results showed that maternal Cognitive and Metacognitive support was rated significantly higher at $\mathrm{T} 2$ compared to $\mathrm{T} 1$ for both cognitive domains (Visual-spatial tasks, $t(34)=-5.884, p<.01$ and for the Language tasks, $t(34)=-3.248, p<.01)$. Maternal Emotional and Motivational support was rated significantly lower but only for the Language tasks $[t(34)=2.377, p<.05$, for the Visual-spatial tasks $t(34)=1.365, p>.05]$.

The Wilcoxon signed rank test was conducted for maternal Contingency and maternal Autonomy support. The results showed that there was no significant difference between maternal Contingent behaviours from T1 to T2 (for the Visual-spatial tasks: $Z=-1.746, p>$ .05 . For the Language tasks: $Z=-.953, p>.05)$. The same results hold for maternal Autonomy support with no significant change at the two-time points (for the Visual-spatial tasks: $Z=-1.823, p>.05$, Language tasks: $Z=-.187, p>.05)$.

Concerning children's SRL skills, the result showed that children's Cognitive and Metacognitive skills were rated significantly higher at T2 compared to T1 (for the Visualspatial tasks: $Z=-4.099, p<.001$, for the Language tasks: $Z=-3.057, p<.001$ ). Children's Emotional and Motivational skills did not show significant change (for the Visual-spatial tasks: $Z=-.884, p>.05$, for the Language tasks: $Z=-.619, p>.05)$. Children's Autonomy skills did not differ from T1 to T2 (for the Visual-spatial tasks: $Z=-1.703, p>.05$. For the Language tasks: $Z=-1.644, p>.05)$.

Overall, these results suggest that for both mothers and children, only the Cognitive and Metacognitive support/skills increased significantly in six months while the other measures remained relatively stable over the six months period. 


\subsection{Differences in the Measures According to the Nature of the Task}

Paired t-tests (for the parametric variables) and the Wilcoxon signed rank test (for non-parametric variables) were conducted to examine whether there was a statistically significant difference in maternal supportive and contingent behaviours and children's SRL skills according to the nature of the task.

As the scores of maternal Cognitive and Metacognitive support (T1 and T2) and maternal Emotional and Motivational support (TI and T2) were normally distributed in both cognitive domains, paired t-test was chosen for these variables. The results showed that mothers provided significantly more Cognitive and Metacognitive support to their children in the Visual-spatial tasks than in the Language task at Time $1, t(34)=4.014, p<.001$ and at Time 2, $t(34)=3.770, p<.01$. Maternal Emotional and Motivational support was reported as significantly higher in the Visual-spatial tasks compared to the Language task but only at Time 2, $t(34)=2.784, p<.01$. For Time $1, t(34)=.902, p>.05$.

The Wilcoxon signed rank test was then conducted for maternal Contingency and maternal Autonomy support between the two cognitive domains. The results showed that there was a significant difference in maternal Contingent behaviours with mothers being rated as more contingent in their support in the Visual-spatial tasks compared to the Language task at both research times (for Time $1: Z=-2.936, p<.01$. For Time $2: Z=-2.187, p<.05$ ). Maternal Autonomy support was not significantly different in the Visual-spatial tasks compared to the Language task at both research times (for Time 1: $Z=-.487, p>.05$. For Time 2: $Z=-1.695, p>.05)$.

Concerning children's SRL skills, the result showed that children's SRL skills were recorded to be used significantly higher in the Visual-spatial tasks compared to the Language tasks at both research Times. For the Cognitive and Metacognitive skills in the Visual-spatial and Language tasks at Time $1: Z=-4.137, p<.001$, for the Cognitive and Metacognitive skills in the Visual-spatial and Language tasks at Time 2: $Z=-4.163, p<.001$. For the Emotional and Motivational skills in the Visual-spatial and Language tasks at Time 1: $Z=-$ 4.099, $p<.001$, and for Emotional and Motivational skills in the Visual-spatial and Language tasks at Time 2: $Z=-4.354, p<.001$. For the Autonomy skills in the Visual-spatial and Language tasks at Time 1: $Z=-4.225, p<.001$, and for Autonomy skills in the Visual-spatial and Language tasks at Time 2: $Z=-3.109, p<.01$. 
Overall, these results suggest that mothers and children were rated higher in their supportive behaviours (except for Autonomy support) and SRL skills respectively in the Visual-spatial tasks rather than in the Language task.

\subsection{Review of the Results}

This section summarizes the main findings of this study. From all the maternal and children's demographic variables and characteristics explored in this study, maternal education was significantly and positively associated with children's SRL skills and performance at Time 1. Children's age was significantly associated with maternal Contingency at Time 1 and Time 2 and with Autonomy support at Time 1 in the Visualspatial tasks. Children's age was also significantly and positively related to most of the children's SRL skills at both research times and tasks. In general, no significant results were found for mothers' and children's characteristics.

The results from both research times highlighted the significance of children's Cognitive and Metacognitive skills at predicting children's performance in both types of tasks. Of the children's distinct groups of SRL skills, Cognitive and Metacognitive skills were the most important factor for children's performance.

Considering the relations between the different maternal aspects of maternal support and children's SRL skills and performance, the results differed for each time of the research and type of tasks. However the study pointed out three main factors that affected children's SRL skills and performance, that is, children's age, maternal Contingency and maternal Autonomy support. In the Visual-spatial tasks at Time 1, maternal Contingency and children's age predicted children's Cognitive and Metacognitive skills and maternal Autonomy support predicted children's Emotional-Motivational and Autonomy skills. In the Language task at Time 1, maternal Contingency predicted children's Cognitive and Metacognitive skills and maternal Autonomy support and children's age predicted children's performance. In the Visual-spatial task at Time 2, children's age and maternal Autonomy support predicted children's Cognitive and Metacognitive skills and maternal Autonomy support predicted children's Emotional-Motivational and Autonomy skills. In the Language task at Time 2, maternal Autonomy support and children's age predicted all of the children's SRL skills and performance.

Mediation analyses for Time 1 showed that in some cases maternal Contingency and maternal Autonomy support partially mediated the relations between children's age and some 
of the children's SRL skills and performance. The most consistent result, however, of the mediation analyses in general, refers to the mediating role of children's Cognitive and Metacognitive skills in the relation between maternal Autonomy support and Contingency and children's performance. This finding implies that the maternal provision of Autonomy support and Contingent support help children to employ more Cognitive and Metacognitive skills which in turn lead to greater performance.

Regarding the short-term longitudinal results, significant results occurred only for the Visual-spatial tasks. Children's Cognitive and Metacognitive skills (T1) predicted children's Cognitive and Metacognitive skills (T2) and performance (T2). As at Time 1 and Time 2, maternal Contingency and maternal Autonomy support were positively linked to most of the children's SRL skills and performance. Maternal Autonomy support (T1) predicted children's Emotional and Motivational skills (T2) and Autonomy skills (T2), and maternal Contingency (TI) predicted children's performance (T2).

The findings also revealed that maternal perceived parenting style, maternal perceived children's temperament and maternal general self-efficacy were not significantly related to the main variables of this study.

Finally, the last result of this study refers to the effect of the nature of the task on the maternal and children's measures. In sum, it seems that in the Visual-spatial tasks in comparison to the Language task, both the mothers and the children in this study were rated higher in their supportive behaviours (except for Autonomy support) and SRL skills respectively. 


\section{Chapter 5 Discussion}

The main research aims of the current thesis were to investigate the actual interactions between mothers and their children during joint problem-solving and to explore the relations between the maternal supportive and contingent behaviours, children's SRL skills and cognitive performance both concurrently and longitudinally. Different maternal supportive behaviours (Cognitive-Metacognitive, Emotional-Motivational, Autonomy support) during joint problem-solving were investigated with a focus on their relations to children's SRL skills and cognitive performance. Moreover, children's and mothers' demographic factors (maternal education, children's age and gender) and characteristics (children's temperament, maternal parenting style, maternal self-efficacy) and their relations to the maternal supportive and contingent behaviours, children's SRL skills and cognitive performance were examined. Finally, this thesis also investigated whether the nature of the tasks differentiated the level of mothers' support and children's SRL skills.

This chapter is comprised of ten sections in which the research findings are discussed and compared with the existent literature. The first section of this chapter provides interpretations of the correlational outcomes found between the central variables of the study and the demographic variables of the participants (e.g., education, age, temperament, etc.). In the second section, the discussion focuses on the associations between the main variables of the study at Time 1 and Time 2. In particular, the results of the relations between children's SRL skills and cognitive performance are presented as well as the relations between the maternal supportive and contingent behaviours, children's SRL skills and cognitive performance. The third section focuses on the relations between the main variables of the study and maternal and children's characteristics. In the fourth section, the longitudinal relations between children's SRL skills (T1), children's SRL skills (T2) and cognitive performance (T2) are discussed as well as the longitudinal associations between the maternal supportive and contingent behaviours (T1) and children's SRL skills and cognitive performance (T2). The fifth section of the chapter presents and interprets the findings of the mediation analyses for both research times and from Time 1 to Time 2 . The sixth section deals with the development of the maternal supportive behaviours and children's SRL skills over a six months period. In the seventh section, the effects of the nature of the task on the examined variables are discussed. An overview of the findings is presented in the eighth section of the chapter. Finally, the contributions and the limitations of the study will also be discussed in the ninth and tenth section respectively. 


\subsection{Children's and Mother's Demographic Variables}

One of the research aims of this study was to examine whether children's and mother's demographic variables were related to the maternal supportive and contingent behaviours and children's SRL skills and cognitive performance. We expected that maternal education and children's age should be significantly and positively correlated with the maternal supportive and contingent behaviours $\left(\mathrm{H}_{1 \mathrm{a}}\right)$, children's SRL skills $\left(\mathrm{H}_{1 \mathrm{~b}}\right)$ and cognitive performance $\left(\mathrm{H}_{1 \mathrm{c}}\right)$. For children's gender, no specific hypothesis was made, due to the lack of evidence for this age of the children and these particular types of tasks.

With regard to mothers' and children's demographic characteristics, the current study detected no evidence for a link between maternal education and the maternal supportive and contingent behaviours. However, the analysis revealed some significant correlations between maternal education and children's SRL skills and cognitive performance at Time 1 for both cognitive domains. Concerning children's age and its relation to the maternal behaviours and children's skills, the study found significant correlations with maternal Contingency at almost both research Times and tasks and with maternal Autonomy support at Time 1 in the Visualspatial tasks. Children's age was also significantly associated with nearly all of the children's SRL skills and cognitive performance at both research Times and tasks. Therefore, the hypotheses $\mathrm{H}_{1 \mathrm{a}}, \mathrm{H}_{1 \mathrm{~b}}$ and $\mathrm{H}_{1 \mathrm{c}}$ were partially confirmed. Finally, considering children's gender, the findings did not show significant results except for maternal Contingency and maternal Autonomy in the Language tasks at Time 2; mothers were rated higher in their Contingent behaviours and Autonomy support towards the girls compared to the boys.

In general, this study did not find positive relations between maternal education level and maternal supportive and contingent behaviours as well as a consistent relation between maternal education and children's SRL skills. This outcome seems in contrast to that of a group of studies which have underlined the positive contribution of maternal education in children's learning outcomes, executive function and maternal behaviours (e.g., Ardila et al., 2005; Korat, 2009; Thompson \& Foster, 2014). However, at the same time, there are also few studies that did not trace such kind of links. For instance, the studies of Lowe et al. (2013, 2014) found no differences in maternal scaffolding behaviours (e.g., providing strategies, defining cause and effect) according to the educational level of mothers of children born with normal birth weight. On the contrary, maternal education affected the scaffolding behaviours of mothers whose children were born with very low birth weight. The study of Meins, Fernyhough, Fradley, and Tuckey (2001) also reported that maternal education was not 
related to some maternal practices, such as sensitivity, responsiveness to infant's objectdirected action, imitation of infant's voices, autonomy support, proper mind-related remarks and infants' Bayley Scale scores. Even if the above studies provide some backing to our findings, it must be highlighted that the limited number of the participant mothers of this study came from high educational background (the majority hold a university degree). This fact could also explain the reason why the supportive behaviours of this group of mothers and children's SRL skills were not significantly differentiated as a function of the maternal educational background. Future research with large sample sizes and a more varied maternal educational profile should examine mother-child interactions during problem-solving and whether maternal education has a significant role in mothers' behaviours, especially in this young age of children. It is possible that these particular associations not to be noticeable yet and to become more apparent as children grow up. Future research should address this issue.

With regard to children's age, the findings of this study are, for the most part, in agreement with the literature. Children's age ranged from 54 to 72 months at Time 1 and respectively 59 to 77 month at Time 2. The findings indicated that children's age was consistently and significantly related to maternal Contingency and to most of the children's SRL skills and performance. This outcome implies that the older was the child, the more contingent the behaviour of his/her mother was during problem-solving. It seems that with older children, mothers were progressively more able to better attune their behaviours with their children's needs. This finding is in line with those of Conner and Cross (2003), who commented that parents when interacting with their children for a longer time, they can better understand and assess the children's abilities. Older and therefore more experienced children can facilitate their parents' understanding of their need for support or independence.

The same positive relations were also found in this thesis regarding the associations between children's age and children's SRL skills. This result was expected as SRL skills are more evident as children grow up (Zimmerman \& Schunk, 2011). The results are also in agreement with the study of Zhang and Whitebread (2017) who found significant positive correlations between children's age and children's metacognitive strategic behaviours, motivational strategic behaviours and task performance. The above findings are of particular importance as they suggest that when examining very young children's efforts to regulate their learning, their age should be taken into consideration even if there is a few months difference.

Regarding children's gender and its relations to maternal supportive and contingent behaviours as well as children's SRL skills and performance, this study, in general, provided 
no evidence of this kind of association. This finding corroborates previous research which did not detect differences in parental behaviours and children's SRL skills and performance according to children's gender. For instance, Neitzel and Stright (2003) found no significant differences in maternal scaffolding behaviours (e.g., metacognitive content, regulating task difficulty, monitoring) in boys and girls, neither did they report such kind of differences in children's self-regulatory behaviours (metacognitive talk, task persistence, help seeking). Similarly, Carr and Pike (2012) did not found gender differences. Zhang and Whitebread (2017), however, reported significant differences in Chinese children, with girls employing more cognitive, metacognitive and motivational strategic behaviours than boys. Studies with older children also mentioned mixed findings (e.g., Hong, Peng \& Rowell, 2009; Wolters \& Pintrich, 1998). Further longitudinal research is needed to determine whether and when parents and children employ different behaviours and skills as a function of children's gender. The cultural background should also be taken into consideration.

\subsection{Cross-Sectional Findings: Time 1 and Time 2}

\subsubsection{Relations between children's SRL skills and cognitive performance}

Another goal of the present study was to investigate the relations between children's SRL skills and cognitive performance at both research times. We expected that children's SRL skills should be significantly and positively associated with their cognitive performance at both research times and tasks $\left(\mathrm{H}_{2}\right)$. We also assumed that children's Cognitive and Metacognitive skills should have the strongest correlations with performance. The findings were consistent for both type of tasks and both research times. Children's SRL skills were positively and significantly related to children's performance. As it was expected, children's Cognitive-Metacognitive skills were the only independent predictor of children's cognitive performance. Therefore, the hypothesis $\mathrm{H}_{2}$ was fully confirmed. Although the children of our study were rated higher in their Emotional-Motivational and Autonomy skills (persistence, concentration, autonomy) compared to their Cognitive-Metacognitive skills, it was the latter that played the most significant role on their performance at both cognitive domains. The Cognitive and Metacognitive strategies employed by the participant children in both cognitive domains, such as analysing and combining activities, planning and monitoring were fundamental for their cognitive performance.

This thesis investigated cognitive, metacognitive and motivational/volitional aspects of SRL and found significant positive correlations with children's cognitive performance. 
The critical role of SRL skills in academic settings has been previously well established (Boekaerts, 1999; Borkowski et al., 2000; Dermitzaki et al., 2008; Rimm-Kaufman et al., 2009; Zimmerman \& Schunk, 2008). The importance of children's Cognitive and Metacognitive skills on their performance has also been supported by other studies. For instance, Dermitzaki et al. (2009) found that children's cognitive and metacognitive skills, in contrast to motivational/volitional skills, directly affected children's performance. Zhang and Whitebread (2017) indicated that children's metacognitive strategic behaviours directly predicted children's performance. Bakracevic, Vukman, and Licardo (2010) have also studied the contribution of cognitive, metacognitive, motivational and emotional self-regulation on students' performance in three age groups (14-15, 17-18, and 22-23) and found that metacognitive self-regulation was a constant and the strongest predictor of achievement. The same study showed that motivational self-regulation contributed less but significantly to children's performance in the first two age groups. Bahri and Corebima (2015) also found that the metacognitive skills contributed more to the cognitive learning outcome than learning motivation (e.g., attention, confidence, and satisfaction).

One possible explanation about why children's Cognitive-Metacognitive skills and not Emotional-Motivational skills were found to be closely related to cognitive performance may also be detected in what Wolters (2003) mentioned about the different purposes served by these regulatory activities: "Strategies for the regulation of cognition are meant to affect students' use of cognitive learning strategies or how students are processing, constructing, or understanding the material with which they are interacting. In contrast, strategies for regulating motivation ultimately are meant to affect students' willingness to process information, to construct meaning, or to continue working. Strategies for regulating motivation do not necessarily influence how students are completing activities, but why they are completing them, or for how long they are completing them" (p. 192).

Considering that the development of SRL begins early in time (even though it is not always apparent) and the fact that too little attention has been paid to young children's efforts to regulate their learning, the findings of this study contributes significantly to the existence knowledge of the links between SRL skills and performance during children's preschool age. This study provided new observational data captured during actual-online episodes, in a more naturalistic setting, and in young children, offering new insights into this particular area of research. 


\subsubsection{Maternal supportive and contingent behaviours and children's SRL skills and cognitive performance}

The present study was designed to determine the relations between different maternal supportive behaviours and contingency to various children's SRL skills and cognitive performance. Based on previous studies, we hypothesised (H3a, H3b, H3c and H3d) that maternal Contingency, maternal Cognitive-Metacognitive support, maternal EmotionalMotivational support and maternal Autonomy support should be positively linked to children's Cognitive-Metacognitive, Emotional-Motivational and Autonomy skills and cognitive performance at both research times and tasks.

The findings from Time 1 showed that two of the maternal variables were related to children's SRL and cognitive performance. Maternal Contingency was significantly correlated with all of the children's SRL skills and cognitive performance in the Language task, and with children's Cognitive-Metacognitive skills in the Visual-spatial tasks (controlling for children's age). In addition, maternal Autonomy support was positively and significantly related to all of the children's SRL skills in both types of tasks, and to children's performance in the Language task. The results of the regression analyses in the Visual-spatial tasks at Time 1 indicated that maternal Contingency and children's age were independent predictors of children's Cognitive-Metacognitive skills and that maternal Autonomy support significantly and independently predicted children's Emotional-Motivational skills and Autonomy skills. As mentioned in the chapter of the results (section 4.4.1), the findings of the regression analyses concerning children's Cognitive-Metacognitive skills and EmotionalMotivational skills should be interpreted with caution due to the $p$ values of the biascorrected and accelerated confidence intervals for B. For the Language tasks, maternal Contingency was an independent predictor of children's Cognitive and Metacognitive skills while maternal Autonomy support and children's age significantly predicted children's cognitive performance in this cognitive domain. Contrary to our expectations, maternal Cognitive-Metacognitive support and maternal Emotional-Motivational support did not show any significant correlations with children's skills and cognitive performance.

A more complex network of closer relations was observed at Time 2. The findings indicated significant and positive correlations between maternal Contingency and Autonomy support with all of the children's SRL skills and cognitive performance in both types of tasks. Maternal Cognitive-Metacognitive behaviours did not show any significant correlations with children's SRL skills. Maternal Emotional-Motivational support was positively and 
significantly related to children's Emotional-Motivational and Autonomy skills but only in the Language task. Further regression analyses showed that in the Visual-spatial tasks, children's age and maternal Autonomy support were significant predictors of children's Cognitive-Metacognitive behaviours and that maternal Autonomy support significantly and independently predicted children's Emotional-Motivational and Autonomy skills. As mentioned in the chapter of the results (section 4.5.1), the findings of the regression analyses concerning children's Cognitive-Metacognitive skills and Emotional-Motivational skills should be interpreted with caution due to the $p$ values of the bias-corrected and accelerated confidence intervals for B and the 95\% Bias Corrected and Accelerated Confidence Intervals that contained zero for maternal Autonomy support. In the Language task, maternal Autonomy support and children's age significantly and positively predicted all of the children's SRL skills and performance. Hence, our hypotheses (H3a, H3b, H3c and H3d) were only partially confirmed.

The above findings have significant implications as it seems that progressively, as time goes by, and according to the type of cognitive task, children's SRL skills benefit from different aspects of maternal support. At Time 1, maternal Contingency was an important predictor of children's Cognitive and Metacognitive skills both for the Visual-spatial and Language tasks. In addition, maternal Autonomy support contributed significantly to children's Emotional-Motivational skills and Autonomy skills in the Visual-spatial tasks and to children's performance in the Language tasks. At Time 2, maternal Contingency although contributed to the general regression models, appeared not to play a central role, as an independent predictor of children's skills. Instead, maternal Autonomy support played a more prominent role for almost all of the children's skills and performance in both cognitive domains.

This study assessed maternal Autonomy support as the mean score of two maternal behaviours, that is Boosting the child's Autonomy and Active Involvement and Overcontrolling (reverse coding). The extent to which the mother encouraged the independent involvement of the child in the problem-solving process, using prompts, hints and questions and the extent to which the mother intervened or not to control the child's effort despite the fact that the child could work independently, appeared to be of particular importance in promoting children's SRL skills. Mothers' ability to gradually hand over the solution process to their children, providing fewer instructions when it was needed and allowing their children to take on more responsibilities emerged to be the most significant maternal behaviour in this study for fostering young children's SRL skills. 
Maternal Contingency, which was more apparent at Time 1, referred to the degree that the mother perceived and responded successfully to her child's real cognitive needs, by attuning and coordinating her responses with the level of the child's understanding. Wood and Middleton (1975) outlined the significance of parental contingency, referring to two particular concepts, that of the "region of sensitivity" and the "contingent shift principle". This thesis indicated that the mother's ability to acknowledge what parts of the task could be managed and completed (or not) by the child (the "region of sensitivity") and her ability to increase her support when the child was facing difficulties, and gradually remove the support when the child succeeded (the "contingent shift principle") were critical patterns of interaction between the mothers and their children, especially at Time 1 when mothers and children were dealing for the first time with a new examination procedure and novel, for them, tasks. The prominent role of parental contingency is also underlined in the study of Zhang and Whitebread (2017) where, among the parental dimensions assessed, only parental contingency was found to independently predict children's strategic behaviours.

Our findings that maternal Contingency and maternal Autonomy are significant factors for children's SRL skills and cognitive performance are in line with those of previous research. Even though these factors are not usually examined simultaneously, both of them have been found to be consistently and positively linked to children's learning outcomes (e.g., Bernier et al., 2010; Mih, 2013; Pino-Pasternak et al., 2010; Zhang \& Whitebread, 2017). Taking these two maternal dimensions into account, this study underlined that maternal supportive behaviours during learning activities are useful for the children's skills if they are provided in a contingent manner (according to the child's level of understanding) that also encourages children's active involvement in the solution process.

Contrary to our expectations, maternal Cognitive-Metacognitive and EmotionalMotivational support were not linked to children's SRL skills and performance. Some studies in the literature, when examining these factors, have highlighted their valuable supporting role in children's learning (e.g., Fender et al., 2010; Puustinen et al., 2008; Robinson et al., 2009; Salonen et al., 2007; Stright et al., 2009). The different results mentioned in the literature are likely to be related to the diverse methodological approaches and tools used in previous research and to the similar but different concepts examined. Most of the studies have addressed different aspects of maternal cognitive-metacognitive (e.g., directiveness, manner of instruction) and emotional-motivational support (e.g., warmth, responsiveness, sensitivity) as well as different children's learning skills (e.g., mathematical and reading skills, executive function), in different time points (e.g., school-age children, adolescents). 
Many of these studies have also used different ways to assess these behaviours (e.g., observation in the classroom, teachers' or parents' reports). As this study used a microanalytic approach to record maternal supportive behaviours and children's SRL skills during problem-solving in young children, more research is needed in this specific area to shed more light on these relations. Another possible explanation may be that the effects of parental supportive behaviours on children's skills at a very young age have not been established yet. The regularity and quality of these interactions are expected to influence the child's cognitive skills, motivation and achievement over time as some longitudinal studies confirm (e.g., Dieterich, Assel, Swank, Smith, and Landry, 2006; Taylor, Anthony, Aghara, Smith, \& Landry, 2008). So, it may be too early to expect stable and one-to-one correlations between parental behaviours and children's skills. Long-term studies that will examine these relationships over more extended time periods could better interpret such associations.

\subsection{Children's and Mothers' Characteristics: Relations to Maternal Supportive and Contingent Behaviours and Children's SRL Skills and Cognitive Performance}

The hypotheses H4a, H4b, H5a, H5b, H6a and H6b of this study regarding children's and mothers' characteristics stated that maternal authoritative parenting, maternal selfefficacy, and children's effortful control should be significantly and positively linked to the maternal supportive and contingent behaviours, children's SRL skills and cognitive performance. In general, no significant associations were found in this study. Therefore, the above hypotheses were not confirmed.

Regarding the maternal parenting styles and their relation to the main variables of this study, the findings did not support those of previous studies which indicated a positive link between an authoritative parenting style and positive parental behaviours or children's academic skills (e.g., Aunola et al., 2000; Berk \& Spuhl, 1995; Boon, 2007; Leung \& Kwan, 1998; Mattanah et al., 2005; Pratt et al., 1992). These differences in the findings could be explained in part by the different methodologies followed to assess parenting styles, parental behaviours and children's skills. For example, some studies have used adolescents' selfreports, other used observational approaches and interviews to assess parenting styles. Different aspects of parenting styles were reported (e.g., positive parenting, harsh parenting, psychological control) as well as different aspects of children's skills (e.g., academic, mathematics and reading, intrinsic motivation and self-efficacy). The present study examined mothers' self-reports about their parenting style and tried to examine possible associations 
with specific groups of behaviours and children's distinct SRL skills during problem-solving. It is possible that the different assessments of parenting styles and the use of different aspects of children's and parents' behaviours to account for the different findings. In any case, our knowledge about the links between parenting styles as assessed by parents and parental supportive behaviours and children's SRL skills as employed during problem-solving is still limited. This is an important issue for future research. Future studies should provide more data on this topic.

Regarding maternal self-efficacy, the literature general reports positive links between parental self-efficacy and general parental practices as well as children's skills (e.g., Ardelt \& Eccles, 2001; Coleman \& Karraker, 2003; Hill \& Bush, 2001; Steca et al., 2011). However, very few evidence is available regarding the relation of maternal self-efficacy with specific maternal supportive behaviours and specific children SRL skills during joint problemsolving. On the other hand, there are also a few studies that did not detect associations between parental self-efficacy and parenting behaviours. For example, Hess, Teti, and Hussey-Gardner (2004) found that maternal self-efficacy was not directly related to maternal behavioural competence (e.g., enjoyment/pleasure, quality and quantity of involvement, amount of quality of visual contact and verbalizations). In addition, Leerkes and Crockenberg (2002) did not discover direct links between maternal self-efficacy and maternal sensitivity. Halpern and McLean (1997) also reported no correlations between maternal self-efficacy and maternal play competence (affect and behaviour towards the infant such as patience, expressions of positive feelings, responsivity). In the case of the relations between parental self-efficacy and children's outcomes, some studies have mentioned indirect effects (e.g., Ardelt \& Eccles, 2001; Purdie et al., 2004; Shumow \& Lomax, 2002). At this point, it should be noted that the assessment of maternal self-efficacy in this study did not refer to the specific mother-child problem-solving tasks, which would probably generate different results. Maternal self-efficacy was assessed at a general level in contrast with the maternal behaviours that were assessed at a task-specific level. Therefore, further work is required to determine the links between maternal self-efficacy with reference to particular problemsolving tasks, specific maternal supportive behaviours and children's SRL skills.

Concerning children's temperament and the relations with the maternal supportive behaviours and children's SRL skills, the findings were not consistent with those of some past studies (e.g., Dixon \& Smith, 2000; Fagot \& Gauvain, 1997, Laake \& Bridgett, 2014). The literature has reported both unidirectional and bidirectional relations between children's temperament and parents' behaviours (e.g., Cipriano \& Stifter, 2010; Eisenberg et al., 2010, 
Feldman et al., 1997; Kochanska et al., 2010). Past studies have also provided information about the links between children' temperament and general academic skills, such as reading skills, arithmetic and language skills (e.g., Deater-Deckard et al., 2009; Noel et al., 2008). Even though past research has paid attention to children's personality traits and SRL skills (e.g., Bidjerano \& Dai, 2007; Busato et al., 1999; Eilam et al., 2009), our knowledge of how children's temperament (effortful control, negative affectivity, surgency) is associated with specific children's SRL skills employed during problem-solving is still unclear. The above studies have examined mostly the relations between children's temperament and academic skills or the relations between children's personality and SRL skills. In addition, the discrepancies noticed between our results and those of other studies could also be attributed to the fact that different methodological tools have been used to assess these factors and different aspects of children's temperament have been examined (e.g., extraversion, neuroticism, soothability, positive emotionality, difficult temperament). Future research should examine these associations with larger samples, taking into account the directions of these links.

\subsection{Longitudinal Findings}

\subsubsection{Relations between children's SRL skills at Time 1, children's SRL skills at Time 2, and cognitive performance at Time 2}

This study also examined the short-term longitudinal relations between early children's SRL skills (T1) and later children's SRL skills (T2) and cognitive performance (T2). It was expected $\left(\mathrm{H}_{7 \mathrm{a}}, \mathrm{H}_{7 \mathrm{~b}}\right)$ that children's SRL skills at Time 1 should significantly predict part of the variance of children's SRL skills and cognitive performance at Time 2 in both cognitive domains. These hypotheses were confirmed only for the Visual-spatial tasks. The findings showed that children's early Cognitive-Metacognitive and EmotionalMotivational skills (Time 1) were significantly and positively correlated with all of the children's later SRL skills and cognitive performance (Time 2) except for Autonomy skills (T2). Children's early Autonomy skills (Time 1) were significantly and positively correlated with children's later Cognitive-Metacognitive and Emotional-Motivational skills (T2). Further regression analyses indicated that only children's early Cognitive-Metacognitive skills (T1) independently predicted children's later Cognitive-Metacognitive skills (T2) and cognitive performance (T2). 
The longitudinal findings are in agreement with those at Time 1 and at Time 2, concerning the primary and fundamental role of children's Cognitive and Metacognitive skills in their cognitive performance. As it was indicated by the results of this study, in the Visual-spatial tasks children's Cognitive and Metacognitive skills at Time 1 independently predicted children's cognitive performance both concurrently and longitudinally. In the Language tasks, although the regression model was statistically significant, neither children's Cognitive and Metacognitive skills (T1) nor their age independently contributed to the model. Therefore, the Hypotheses $\mathrm{H} 7 \mathrm{a}$ and $\mathrm{H} 7 \mathrm{~b}$ were only partially confirmed.

The above findings are in agreement with some studies that have also reported significant longitudinal associations between children's SRL skills, executive function skills and later achievement (e.g., Blair \& Razza, 2007; Fuhs, Nesbitt, Farran, \& Dong, 2014; McClelland, Acock, \& Morrison, 2006; Welsh, Nix, Blair, Bierman, \& Nelson, 2010). Blair and Razza (2007) examined the role of some aspects of executive function assessed in preschool and kindergarten in math and literacy ability in kindergarten. Discussing the apparent relations between children's executive function and mathematics ability, the researchers explained that: "Proficiency in mathematics at all levels requires the individual to reason actively about problem elements when arriving at possible solutions. The problemsolving process requires the individual to represent information in working memory, to shift attention appropriately between problem elements, and to inhibit a tendency to respond to only the most salient or most recent aspect of a given problem", "Even for very young children, however, mathematics is inherently effortful and makes explicit demands on executive function abilities" (pp. 658-659). About the relations between executive function and some aspects of emergent literacy, the researchers explained that "In contrast to mathematics, phonemic awareness and letter knowledge are aspects of emergent literacy that are becoming less effortful and more automatic as children develop", "perhaps letter identification and phonemic awareness abilities make demands of executive function as this knowledge is being acquired" (p. 659). The findings of this study suggested that executive function has a role in mathematics, phonemic awareness and letter knowledge, but in mathematics, this role is more substantial. The differences noticed in the two types of tasks of the present study will be analysed further in a following section. 


\subsubsection{Maternal supportive and contingent behaviours (T1) and children's SRL skills}

\section{(T2) and cognitive performance (T2)}

The hypotheses for the longitudinal relations between the early maternal supportive and contingent behaviours (T1) and children's later SRL skills (T2) and cognitive performance (T2) stated that the maternal supportive and contingent behaviours at Time 1 should significantly predict part of the variance of children's SRL skills and cognitive performance at Time 2 in both cognitive domains $\left(\mathrm{H}_{8 \mathrm{a}}\right.$ and $\left.\mathrm{H}_{8 \mathrm{~b}}\right)$. The findings of the shortterm longitudinal study showed that significant results emerged only for the Visual-spatial domain regarding the links between aspects of the maternal supportive and contingent behaviours at Time 1, children's SRL skills at Time 2 and cognitive performance at Time 2. In particular, maternal Contingency (T1) was significantly and positively related to children's Cognitive and Metacognitive skills (T2) and cognitive performance (T2) while maternal Autonomy support (T1) was positively and significantly correlated with all of the children's SRL skills (T2). The regression analyses supported that maternal Autonomy support (T1) was an independent predictor of children's Emotional-Motivational and Autonomy behaviours (T2), while maternal Contingency (T1) independently predicted children's cognitive performance (T2). With reference to the Language domain, no significant associations were observed.

Regarding the Visual-spatial tasks, this study points out the importance of early maternal Contingency and Autonomy support in children's later SRL skills and performance. The findings are in accordance with previous studies that detected longitudinal associations between parental behaviours and children's skills (e.g., Cheng, Lu, Archer, \& Wang, 2017; Dieterich et al., 2006; Hammond et al., 2012; Hubbs-Tait, Culp, Culp, \& Miller, 2002; Taylor et al., 2008; Smith, Landry, \& Swank, 2000; Zeytinoglu, Calkins, \& Leerkes, 2018). However, different aspects of parenting (e.g., contingency, cognitive-emotional-autonomy support, positive feedback) and different aspects of children skills (e.g., cognitive and language skills, perceptual skills, reading or executive function skills) were investigated in most of these studies. Thus, which particular parental supportive behaviours predict longitudinally specific children's SRL skills during problem-solving contexts, in which cognitive domain, is an area of research that needs further documentation and similar methodological tools. Therefore, the Hypotheses H8a and H8b were confirmed only with reference to the Visual-spatial domain and for specific aspects of maternal supportive behaviours. 
An interesting question that emerged from these findings refers to why early maternal Contingent and Autonomy supportive behaviours seem to influence children's later SRL skills only in the Visual-spatial domain and not in the Language domain. These differences between the two cognitive domains were also noticed in the associations between children's early SRL skills (T1) and children's later SRL skills (T2). This study did not find longitudinal relations in the Language task even though the findings at Time 1 and Time 2 had shown significant associations between the maternal supportive and contingent behaviours and children's SRL skills, especially at Time 2.

One possible reason for these results may be found in the tasks' characteristics. In the Visual-spatial tasks mothers and their children were asked to reproduce patterns of cubes assemblies as they were depicted in card-models. This type of task is somehow familiar in parents and children at this age, as it looks similar to many construction games (e.g., puzzles, building tiles). The Visual-spatial tasks were also a more structured activity with more specific steps to be followed in order to come to a solution. On the contrary, the Language task included the development of a story based on given pictures where the mother and the child had to create a story, to connect meanings, to provide a structure for this story, to offer logical explanations and links about how the events unfolded. This type of activity was more abstract than the one in the Visual-spatial tasks. Therefore, one likely explanation why there was a general trend to detect only crossectional relations in the Language tasks could be that this type of task may involve more automatic processes and the demands to specific SRL skills are taken place as the information is being attained (see, also, Blair \& Razza, 2007, section 5.4.1). In addition, the fact that the correlations in the longitudinal study were in general weaker than those examined concurrently for both cognitive domains should also be taken into consideration. This finding could mean that maybe the efforts of the participant mothers to support their children at Time 1 might have been affected by the unfamiliar process and the novelty of the tasks. At Time 2, both the mothers and children knew about the procedure, the tasks' demands and how to better work with each other. Therefore, these first maternal supportive behaviours (at Time 1) in combination with the characteristics of the Language task described above could explain the findings in the longitudinal study. Future research is much needed in this area to provide information about how the demands and characteristics of the task could affect parental behaviours and children's skills. Moreover, the above findings could also be interpreted in combination with the findings of the unit 5.7 on the effects of the nature of the tasks. 


\subsection{Findings from the Mediation Analyses}

Interesting results emerged from the mediation analyses and referred to the effect of children's age on children's use of Cognitive-Metacognitive skills and the effect of maternal Autonomy support and Contingency on children's performance. The mediation analyses at Time 1 showed that the effect of children's age on children's use of Cognitive and Metacognitive skills was partially mediated by the maternal Contingency in both types of tasks. This finding is important as it underlines the significance of maturation and biological processes as well as environmental factors and their interaction on children's learning outcomes. As Kolb and Gibb (2011) argued in their review about "brain plasticity and behaviour in the developing brain" where they studied various factors that affect the development of the brain including parent-child interactions, "The development of the brain reflects more than the simple unfolding of a genetic blueprint but rather reflects a complex dance of genetic and experiential factors that shape the emerging brain" (p. 265). The findings at Time 1 depicted that children's age predicted children's Cognitive and Metacognitive skills both directly but also indirectly through maternal Contingency; in older children mothers were more effective at providing Contingent behaviours which in turn positively affected children's use of Cognitive and Metacognitive skills. It seems that maternal Contingent behaviours are more successful in older children where both the mother and the child have learnt how to attune with each other.

Another finding from the mediation analyses reported that in the Language task at Time 1 and in the Visual-spatial and Language tasks at Time 2, the effect of maternal Autonomy support (and Contingency in some cases) on children's cognitive performance was mediated by children's use of Cognitive and Metacognitive skills. Furthermore, the mediation analyses in the short-term longitudinal study revealed a serial mediation in the Visual-spatial tasks, that is, maternal early Contingency (T1) predicted children's early use of Cognitive and Metacognitive skills (T1), which in turn predicted children's later use of Cognitive and Metacognitive skills (T2), which in turn predicted children's performance (T2) in the Visualspatial tasks. These findings support that in most cases, the supportive behaviours of the participant mothers did not directly affect children's performance; in turn, mothers Contingent and Autonomy behaviours positively affected children's performance by supporting children's Cognitive and Metacognitive skills.

The literature which explores children's SRL skills as a mediator factor between parental behaviours and children's performance is scarce. Few recent studies, however, 
provide such evidence. For instance, similar results were reported by Zhang and Whitebread (2017) who found that children's metacognitive behaviours mediated the relations between maternal contingency and children's achievement. The study of Daniel, Wang, and Berthelsen (2016) reported that the relation between school-based parental involvement at Grade 1 (e.g., visits at school, communication with teachers, etc.) and children's reading and numeracy achievement at Grade 3 was fully mediated by student's SRL skills. Xu, Benson, Mudrey-Camino, and Steiner (2010) also mentioned that children's SRL skills mediated the relation between parental involvement (e.g., parent-child communication, homework help, school involvement, etc.) and reading achievement in the fifth Grade. Therefore, future research should consider further investigation of the role of SRL skills between parenting behaviours and children's performance, providing further information on this matter.

\subsection{Short-Term Longitudinal Observations of Maternal Supportive and Contingent Behaviours and Children's SRL Skills}

This study also explored the short-term developmental course of the maternal supportive and contingent behaviours and children's SRL skills. Overall, maternal Cognitive and Metacognitive support, as well as children's Cognitive and Metacognitive skills, were rated higher in the assessment scale over time for both types of tasks. The other variables did not significantly change over the six-month period (except for maternal EmotionalMotivational support in the Language task which was significantly reduced).

What seems interesting in these findings is that both the mothers and the children of this study were rated higher in their Cognitive and Metacognitive behaviours at Time 2, and yet these two factors were not significantly related to each other. A possible explanation of these findings could be that the maternal acquaintance and familiarity with the tasks and the examination procedure at Time 2 , may have urged mothers to use more challenging talk and instructions during joint problem-solving. As mentioned above (section 5.4.2) mothers at Time 2 were more acquainted with the procedure and with the tasks' demands, and this might have created a more favourable attitude towards the tasks.

Concerning the examination of the development of specific maternal supportive practices in time, the findings in the literature are mixed, possibly because of the different time periods under investigation and the various measures used to assess maternal and children's behaviours. Nonetheless, some differences in maternal behaviours over time were reported in the literature (e.g., Fagot \& Kavanagh, 1993; Plumert \& Nichols-Whitehead, 
1996). However, general conclusions are difficult to be drawn due to the restrictions mentioned above. More longitudinal research is needed in this field to shed light on the trajectories of the maternal supportive and contingent behaviours during joint problemsolving.

In the case of children's SRL skills, the above findings were expected. During children's early development, fundamental changes are happening in their cognitive functioning (Whitebread \& Basilio, 2012). Wigfield, Klauda, and Cambria (2011, p. 34) discussed three essential developments in self-regulation that is, the development of the capacity to regulate (biologically), the development of children's knowledge, strategies and expertise in various areas, and the factors that relate to self-regulation (e.g., self-efficacy, goals, language skills). According to children's age, there are some biological restrains on their self-regulation ability and the refinement of children's knowledge and strategies could be linked to children's age and biological maturation; however, differences can also be detected to children of the same age. Alexander, Graham, and Harris (1998, pp. 137-138), mentioned that as children's experiences increase (familiarity and practice), changes in the development of strategies appear; children are becoming more efficient, their strategic behaviour is more effective, their "strategies are applied in more flexible ways", children are relying less on strategic solutions, and there is a qualitative shift in their strategies. More recently, Bryce and Whitebread (2012) studied the development of metacognitive skills (e.g., monitoring, control behaviours) in five and seven years old children. They found both quantitative and qualitative changes in favour of the older children when task difficulty was controlled. On the other hand, one study that attempted to track the developmental trajectories of children's (5-10 years old) attention focusing and attentional and behavioural persistence reported somewhat stable results for attention and for the most part for persistence (except for children with low persistence at the beginning of the study who later showed a significant increase) (Zhou et al., 2007). The researchers suggested that the children's ability to focus and maintain their attention matures and becomes stable by early to middle years of children's lives.

In conclusion, the findings of the present study regarding children's short-term longitudinal changes in SRL skills are in agreement with past studies and could be attributed to maturation, environmental and educational factors. 


\subsection{Differences in the Measures According to the Nature of the Task}

The last Hypothesis of this thesis stated that the nature of the task should differentiate the level of maternal support and the employment of children's SRL skills $\left(\mathrm{H}_{9}\right)$; moreover, one could assume that some differences in the relations between the variables of the study should emerge as a function of the different cognitive domain they refer to. However, no specific hypotheses were made as different types of tasks, different parental behaviours and children's skills are assessed in literature and, therefore, there are no consistent data. In addition, due to the lack of previous data, a specific hypothesis on the effects of the cognitive domain examined on the relations among the study variables could not be formulated.

The findings revealed that maternal Cognitive and Metacognitive support and maternal Contingency were rated significantly higher in the Visual-spatial tasks. Maternal Emotional and Motivational support was rated higher in the Visual-spatial tasks, compared to the Language task, but only at Time 2. Maternal Autonomy support did not significantly change at both research times. Regarding children's SRL skills, the findings supported that children were rated higher in their SRL skills in the Visual-spatial tasks compared to the Language task at both research times.

The effect of the type of the task on parental behaviours during parent-child interactions has been previously investigated. However, studies usually have used different tasks, such as a photo album, reading a book or home-like activities (e.g., Kermani \& Janes, 1999; Korat, 2009; Aram, 2002). González (1996) reported similar to this study's findings that were attributed to the task demands: parents felt that they had to be more active and to use more commands, questions or declaratives in the more structured tasks (constructions, reading tasks) than in more free-play tasks (play with household objects) maybe because children find the first tasks more challenging. Neitzel and Stright (2004) found that mothers who perceived a task negatively (in terms of its familiarity, interest and difficulty for their child) provided less metacognitive information and regulation of task difficulty and more over-controlling behaviours. The researchers suggested that perhaps feelings of frustration or children's negative behaviours when dealing with unfamiliar, uninterested and difficult tasks had a negative impact on the maternal behaviours during the activities. These findings may stress the need to examine separately maternal and children's perceptions of the tasks, including distinct measures of familiarity, interest and difficulty of the task both for the children and parents. The fact that the participant mothers in this study were rated lower in Contingency and Cognitive-Metacognitive support in the Language task could also be 
attributed to the specific type of Language task used in this study and its demands. Creating and telling a story probably has different language demands compared to reading and discussing a story. In this respect, Curenton, Craig, and Flanigan (2008) found that mothers provided their children with more decontextualized talk during story-telling than during story-reading probably because they were engaged in more discourse in the first activity. Storytelling provides more sophisticated language to children. They also explain that decontextualised language includes discussion about past or future events as well as about abstract concepts or events and requires high order reasoning. Therefore, it is also possible that the mothers and the children of this study were not familiar with the particular type of Language task, and perhaps they were participating in more traditional activities, such as book reading which, however, does not provide the level of skills needed in creating a story

Taking the above into account, it could be supported that the differences noticed between the two cognitive domains regarding the maternal Contingency and CognitiveMetacognitive support could be explained by children's and mothers' perceptions of these tasks. It is possible that the participant mothers might have considered the Visual-spatial tasks to be more structured, familiar, less automatic, and more interesting than the Language task both for them and their children, and, as a consequence, they participated more in the Visualspatial tasks. In addition, maternal beliefs about the value of a task and which type of task is more important for them and their children is another possible interpretation of the above findings. Future studies should take into consideration the nature of the tasks and parental perceptions of the tasks before examining such kind of relations.

The findings of the study also showed that the maternal Emotional-Motivational behaviours at Time 1 and Autonomy behaviours at both research times did not differ significantly with regards to the nature of the task. Perhaps, the level of encouragement and positive commenting the child as well as the level of supporting the child's independent cognitive involvement in the solution process, express more stable maternal characteristics, not so much affected by the context. The literature has reported links between personality traits and parental behaviours such as sensitivity and affection, positive support, warmth and encouragement of autonomy (e.g., Belsky, Crnic, \& Woodworth, 1995; Losoya, Callor, Rowe, \& Goldsmith, 1997). The present study also showed that the maternal EmotionalMotivational behaviours in the Visual-spatial tasks and Autonomy behaviours in both tasks did not change during the six-month period of time (from Time 1 to Time 2), indicating that in most cases the level of maternal Emotional-Motivational and Autonomy support did not differ both between the two research times and between the two different cognitive domains. 
Future research could further elucidate these relations and provide information regarding the development of these behaviours over time.

In the case of children's SRL skills, the findings are in agreement with previous research suggesting that the nature and the type of the task influence the employment of children's learning skills and strategies. Such differences in the employment of SRL skills have also been noticed in various school subjects and disciplines (e.g., Vanderstoep, Pintrich \& Fagerlin, 1996; Wolters \& Pintrich, 1998). The different cognitive demands and the features of the tasks might explain the findings of this study referring to children's higher levels of SRL skills in the Visual-spatial tasks of this study. The specificity of a task has been found to be linked to the employment of higher levels of children's skills, such as asking questions, making comments and active engagement (Kermani \& Janes, 1999). In the same vein, Alexander et al. (1998, p. 144) explaining domain differences in students' strategic processes wrote that "In mathematics, for example, students are frequently exposed to rather well-structured problem-solving tasks, such as long division or multiplication problems. Such well-structured problems are typically tackled by domain-specific strategies that are rather linear and algorithmic. By contrast, the domain of reading is better characterised as an illstructured domain in which learners often cope with amorphous problems, such as identifying or constructing the main idea or summarising text. In such a domain, strategies of choice are frequently general strategies in the form of heuristics. Given the relationship between domains and problem-solving tasks, the influence of the domain in strategy development is, thus, understandable". Except for the specificity and structure of the Visual-spatial tasks, these findings may also be the result of children's interest and familiarity with the material presented to them. Children were more enthusiastic about the Visual-spatial tasks rather than the Language task. They demonstrated a higher level of attention and persistence in the Visual-spatial tasks. Children's interest has been previously linked to achievement, motivational aspects of learning, attentional processes, persistence and positive affect (e.g., Ainley, Corrigan, \& Richardson, 2005; Ainley, Hidi, \& Berndorff, 2002; Hidi \& Renninger, 2006; Schiefele, \& Csikszentmihalyi, 1995). In addition, the Language task needed muchdecontextualised talk, which in the case of non-practice drove children to quickly lose their interest and unsuccessfully deal with this type of task. Future research should examine children's SRL skills in Visual-spatial and Language tasks, including a variety of tasks in both domains, also taking into account children's familiarity and interest. 


\subsection{Overview of the Findings}

This study investigated the role of different aspects of maternal supportive behaviours in preschool children's SRL and, at the same time, it explored the contribution of various factors, such as demographic and personality characteristics in the above relations.

Regarding the links between children's and mothers' demographic variables to the maternal supportive and contingent behaviours, the findings of this study showed that only children's age (and not maternal education) was positively and significantly associated with maternal Contingency at almost both research Times and tasks and with maternal Autonomy support at Time 1 in the Visual-spatial tasks. Concerning children's SRL skills and cognitive performance and their links to children's and mothers' demographic variables, the results depicted that there were positive and significant correlations between maternal education and children's SRL skills at Time 1 for both cognitive domains, and between children's age and children's SRL skills and cognitive performance at both research Times and tasks. Considering children's gender, the results, in general, did not reveal significant associations with the maternal supportive and contingent behaviours or with children's SRL skills and cognitive performance.

This study also examined the relations between children's SRL skills and cognitive performance both cross-sectionally and longitudinally. The findings of the study indicated that at Time 1 and at Time 2 children's SRL skills were positively and significantly associated with children's cognitive performance in both cognitive domains. However, only children's Cognitive and Metacognitive skills uniquely predicted children's cognitive performance concurrently in both cognitive domains and longitudinally in the Visual-spatial tasks.

The cross-sectional and longitudinal findings of the present study have identified two factors as the most significant in promoting young children's SRL skills and performance during joint problem-solving. Maternal Contingency and Autonomy support of the child were found to be the most important practices for pre-school children skills both concurrently and longitudinally. In particular, in the Visual-spatial tasks at Time 1, maternal Contingency and children's age predicted children's Cognitive and Metacognitive skills and maternal Autonomy support predicted children's Emotional-Motivational and Autonomy skills. In the Language task at Time 1, maternal Contingency predicted children's Cognitive and Metacognitive skills and maternal Autonomy support and children's age predicted children's performance. In the Visual-spatial task at Time 2, children's age and maternal Autonomy 
support predicted children's Cognitive and Metacognitive skills and maternal Autonomy support predicted children's Emotional-Motivational and Autonomy skills. In the Language task at Time 2, maternal Autonomy support and children's age predicted all of the children's SRL skills and performance.

To sum up, the results from Time 1 highlighted the importance of the adult's understanding and subsequent attunement of which parts of the tasks are difficult or not for the child so that to provide appropriate levels of support. The tendency of the mothers to increase their assistance (cognitive, metacognitive or emotional) when children show signs of difficulty in handling some parts of the task, and the tendency to withdraw this assistance when children appear to succeed, was critical for children at Time 1. This Contingency in mothers' behaviours was found in this study to be one of the most significant factors that foster children's cognitive and metacognitive behaviours. Perhaps, maternal Contingency was more important at Time 1 as the participants were not acquainted with the examination procedure. Thus, mothers' efforts were more focused on coordinating their behaviours with their children's level of understanding, in an attempt to understand the task characteristics and demands. At Time 2, the mothers and the children were more familiar with the tasks and further acquainted with each other, and might this mother-child coordination has become easier.

In addition, the results from Time 1 and Time 2 pointed out as an important factor the maternal Autonomy support of the child. The fundamental function of maternal autonomy support versus over-controlling behaviours to children's learning is well established in the literature (e.g., Fan \& Williams, 2010; Grolnick, Gurland, DeCourcey, \& Jacob, 2002; Joussemet, Koestner, Lekes, \& Landry, 2005). Furthermore, maternal Autonomy support became more influential at Time 2 compared to Time 1 , in contrast with the effects of maternal Contingency, which was not an independent predictor of children's SRL skills. This finding is important, since maternal Autonomy support was not significantly increased at Time 2 compared to Time 1, but its effect on children's SRL skills was more prominent. This contribution of Autonomy support at Time 2 versus maternal Contingency was more apparent in the Language domain.

Regarding the longitudinal findings of the present study, it has been shown that the two above mentioned maternal dimensions at Time 1 were also critical for children's later SRL skills and performance at Time 2 only in the Visual-spatial domain. As at Time 1 and Time 2, earlier maternal Contingency and maternal Autonomy support were important factors for children's later SRL skills and cognitive performance. Maternal Autonomy support (T1) 
predicted children's Emotional and Motivational skills (T2) and Autonomy skills (T2), and maternal Contingency (T1) predicted children's performance (T2) in the Visual-spatial tasks.

Moreover, this thesis explored the relations between children's and mothers' characteristics with the study's variables. Children's temperament, maternal parenting style and maternal self-efficacy were included. The findings of this study showed no statistically significant correlations neither with the maternal supportive and contingent behaviours nor with children's SRL skills and performance. The differences in the methodological tools used between studies, the various conceptualizations of the terms, and the multifaceted role of these variables in direct and indirect links or as mediators were discussed as possible interpretations of the above findings.

The results of the mediation analyses revealed two significant outcomes. At Time 1 children's age predicted children's Cognitive and Metacognitive skills both directly and indirectly through maternal Contingency. Mediation analyses also showed that mothers' Contingent and Autonomy behaviours had an additional indirect effect on children's cognitive performance through children's Cognitive and Metacognitive skills during problem-solving.

This study also investigated the effect of the nature of the task on the maternal supportive and contingent behaviours and children's SRL skills. The findings supported that the participant mothers were rated higher in Contingency and Cognitive-Metacognitive behaviours in the Visual-spatial tasks compared to the Language tasks. In addition, the participant children were rated higher in all SRL skills in the Visual-spatial tasks compared to the Language task. Children's and mothers' interest on the task, the familiarity with the tasks, the demands and the structure of the tasks, as well as the maternal perceptions of the task have been discussed as factors that might explain the above findings.

\subsection{Contribution of the Study and Practical Implications}

From a theoretical view, the study contributes to our understanding of the way mothers can support their children's early learning experiences. The empirical findings in this study offer a new perspective on how to approach mother-child interaction and children's learning. New data are provided about young children's SRL, a research area that has not received much attention. In addition, this study examined the mother-child problem-solving efforts in two different cognitive domains. This research suggests that when examining joint problem-solving in young children, the time and the nature of the task should be taken into 
consideration as it was shown that these factors were differentially associated to the various aspects of maternal behaviours and children's skills.

Methodologically, the study highlighted the need for more multidimensional studies which will examine parenting behaviours through a broader spectrum. Up to date, studies in this field have used different concepts of parental supportive behaviours and children's SRL skills, assessing different aspects compared to other studies. More often, research has paid more attention to a specific dimension of parenting, such as socio-emotional behaviours or autonomy support, and the links to some children's skills (e.g., delay of gratification, academic skills). Nonetheless, the literature has called for more multidimensional studies that should explore at the same time different aspects of parental behaviours and different aspects of children's skills. This study's unique contribution lies in the fact that it explored different aspects of maternal supportive behaviours and children's SRL skills during on-line problem-solving to examine how these variables unfold and develop through this critical period for children's learning. Such multidimensional studies provide valuable information about which parenting behaviours are the most important for the development of children's learning skills.

Furthermore, very few studies so far have approached at the same time parental behaviours and children's SRL skills by applying a micro-analytic methodology. This research adopted an observational methodology to assess maternal behaviours and children's SRL during joint interactions. Mothers and children were observed during actual episodes that allowed capturing these behaviours and skills the time they were employed in a more naturalistic setting. Further investigations should include a combination of research methods to further analyse maternal supportive behaviours and to examine whether and how they develop over different time periods to support children's learning efforts.

More importantly, this study examined the relations between maternal supportive behaviours and children's SRL skills both concurrently and longitudinally. The crosssectional and longitudinal analysis of these links enhanced the understanding of the way maternal supportive and contingent behaviours were related to children's SRL skills over time. In addition, new evidence was provided about which early maternal supportive and contingent behaviours (Time 1) were more important for the development of children's later SRL skills and performance (Time 2). The findings of the study suggested that over time different aspects of parenting were important to children's SRL skills and this information should be taken into account when investigating parent-child problem-solving, especially in preschool years. 
There is, therefore, a need to further study the frequency and quality of these interactions, as well as a need for reliable tools and a common conceptualisation of maternal behaviours during problem-solving. Valid and reliable tools that allow researchers and educators to capture the actual adult practices at a micro-level are needed. Structured observation tools could be useful in recording and investigating such behaviours enacted during joint learning. A more detailed exploration of these relationships will provide useful insights into how parents and teachers can support children's learning efforts in more effective ways and can help design interventions that encourage the use of specific practices. Furthermore, it is necessary to explore further the relationship between parental characteristics, maternal behaviour and children's learning skills to detect factors that influence these relations.

Practical implications. This study underlines the role of children's SRL skills in their performance, a finding that has been found in academic contexts too. One of the issues emerged is that SRL skills should be an essential element of education both at home and in school settings. During this period of children's development (4.5 to 6 years old), where usually formal education begins, parents and teachers could contribute considerably to children's developing skills. In this line, the present study raises the issue that young children's SRL skills are substantially assisted by particular maternal behaviours emerged during educational and play interactions. The evidence of this research could be a useful source of knowledge about the way parents and teachers can foster SRL skills to their children/students. For instance, this study may suggest that when parents and children deal with cognitive tasks, contingency is more important at the beginning of the problem-solving process. Parents should not be concerned about the level of their support, but about how to adjust their support to their children's level of understanding. When the mother-child pair becomes more familiar with the task characteristics and demands, maternal autonomy support seems to become more significant in fostering children's SRL skills. The fact that the current investigation also pointed out the longitudinal effects of early maternal behaviours to later children's skills should be seriously taken into consideration for early interventions. These maternal behaviours should be the target of intervention programs and the objective of future research.

In particular, the insights gained may be of assistance to educators and educational psychologists to design and implement more focused and efficient educational programs addressed to adults involved in children's learning (e.g., Dermitzaki \& Kriekouki, 2017). The present findings could allow such practical implications. Greek parents are involved in 
children's education especially in the context of homework. The findings regarding the importance of parental Contingency and Autonomy support could be generalised in such settings and provide parents with useful strategies on how to support their children's learning. These findings are also a valuable tool for psychologists, teachers and other practitioners, suggesting that parents should employ more Autonomy supportive behaviours when interacting with their children in learning activities. Considering the concept of Contingency and its significance in children's learning, especially in their early efforts, practitioners should inform and teach parents effective ways to coordinate their instructions with children's cognitive needs. In this vein, the same implications are applied in school settings; teachers should understand the importance of granting autonomy to their students as well as the magnitude of being aware of each child's level of understanding in order to adjust their level of instruction. Both parents and teachers should be supported to better recognise their children's developmental and educational needs in order to provide effective strategies that correspond to the child's level of understanding.

This study took into consideration the limitations of previous research and moved forward to examine both multidimensionally and longitudinally mother-child interactions during problem-solving in two different cognitive domains while examining various factors that affect parenting and children's outcomes. However, to go beyond these relations, future research should utilise the findings and limitations of the present study to develop a better understanding of the factors that affect children's learning skills at each development stage and type of task to effectively support children's developing skills and learning.

\subsection{Limitations and Future Research}

Even though interesting results emerged from the present study, there are some restrictions that should be taken into consideration. The first limitation refers to our sample's characteristics. The fact that the study included a complex micro-analytic and longitudinal design justifies the small number of mother-child dyads participated in the study. However, the scope of this study was limited due to the number of participants. In particular, the small sample size did not allow the inclusion of more predictors in the multiple regression analysis. In addition, further statistical analyses (e.g., path analysis) were not possible to be performed as they require larger sample sizes. Therefore, the nature of the relations examined in this study was mainly correlational, and even if mediation and regression analyses were run, the direction of the relations should be interpreted with caution. Future studies should also 
examine the bidirectional relations between parental supportive behaviours and children's SRL skills.

One more important limitation lies in the participants' characteristics. Specifically, the sample size, the origin of the sample, the educational level and the gender of the parents, yield concerns about the generalization of the findings. The group of the participant mothers consisted of mothers from two cities in central Greece with mainly high educational levels. Mothers with lower educational level or mothers who live in bigger prefectures or inversely in urban areas may interact differently with their children during joint problem-solving. In addition, it is highly possible for fathers to react differently from mothers in problem-solving conditions. The fact that our study included only mothers excluded this hypothesis from further investigation. In addition, the preschool age of the children constrains the findings of the study. The age of the children was found to be related to both mothers' supportive behaviours and children's SRL skills. Therefore, future studies should provide data from older children to investigate how these relations develop later in school.

This study included both demographic and other maternal and child's characteristics to investigate possible factors that affect children's SRL skills. However, it is possible, that other variables could also influence the relations found between maternal supportive and contingent behaviours and children's SRL skills and performance. For example, such possible factors may be mothers' and children's general intellectual abilities, maternal stress, mothers' and children's interpretation of the tasks, as well as teachers teaching and instructional behaviours in the class. These factors were not controlled in our study, but future research should also take into account the impact of those factors on children's SRL skills.

Moreover, because of the sample's limitations, we grouped the initial eight maternal behaviours into three categories, that is, cognitive-metacognitive, emotional-motivational, and autonomy-supportive behaviours. This grouping may have limited the discovery of significant results between other maternal behaviours such as maternal over-controlling and maternal negative criticism and children's skills. These behaviours may have a greater impact on mother-child interaction than the other positive aspects (e.g., encouragement, autonomy support).

The short-term longitudinal design is another factor that constraints our findings. This study included measures only at two-time points (Time 1 and Time 2). Even though this time was enough to reveal significant developmental trajectories, for children especially, it is not enough for general conclusions about how maternal behaviours change during the preschool 
years or whether and how different maternal supportive behaviours affect later children's skills. Therefore, future research should examine these relations longitudinally including more research shots of data gathering over time. This limitation also brings another point, that of the time point and the context of the interactions. This study investigated mother-child interaction during problem-solving tasks at a specific time point. Therefore, conclusions and generalisations about another type of mother-child interaction which is unfolded during longer periods of time (e.g., during homework, everyday tasks, etc.) should be made with much attention.

One last issue that should be noted for this study is the use of the self-report questionnaires for assessing children's temperament, parenting style and parental selfefficacy. The lack of significant results between these factors and the central variables of the study may be due to the small sample size, but also to the limitations emerged by the questionnaires. Self-report questionnaires have low flexibility and are subject to social desirability bias. In addition, the specific scales used in this study measure more general behaviours and traits than those appear in problem-solving conditions. For example, the CBQ instruments assess children's temperament which may be different from the one that appears in specific time-points when children interact with their mothers during problem-solving with the presence of the examiner. The above tools referred to a general and not to a task-specific level, as maternal support and children's skills.

These limitations described above provide insights for future studies. Research exploring these factors should include a large sample size to assure the implementation of more complex statistical analysis. Longitudinal studies could identify the directions between the examined relations. In addition, including more predictors will allow the investigation of which particular factors that act simultaneously have the most important role in supporting children's SRL. Further research is required with samples from different educational and cultural backgrounds that could elucidate how and whether these factors affect parental behaviours or children's skills (e.g., see, Zhang \& Whitebread, 2017 with Chinese parents and children), or if they have a mediation role in the examined relations. Future studies should also consider examining the contribution of fathers' behaviours and general attitudes in children's learning as well as the general effect of both parental supportive behaviours in children's skills and performance.

The issue of the nature of the task is an intriguing one and should be considered for further research. This study showed that the nature of the task differentiates mothers' and children's behaviours. Future studies should investigate parents' and students' behaviours in 
different cognitive domains (e.g., reading a book, puzzles, constructions, watching a movie). Different assessments of the measures should also be taken into account, such as daily observation, observation during other tasks (e.g., watching a video, playing a board game) as well as observation in the classroom to form a more accurate assessment of the actual parental and children behaviours. Finally, longitudinal designs which will track these behaviours for longer time intervals will give essential information of how parental behaviours change over time and how or whether they affect children's SRL. This knowledge will support researchers to establish a better degree of understanding in this matter. Finally, given the fact that this study explored typically developing children, one research area worth examining is how the relations found in this study change, if they do, when parents interact with children with learning difficulties. 


\section{References}

Ainley, M., Corrigan, M., \& Richardson, N. (2005). Students, tasks and emotions: Identifying the contribution of emotions to students' reading of popular culture and popular science texts. Learning and Instruction, 15(5), 433-447.

Ainley, M., Hidi, S., \& Berndorff, D. (2002). Interest, learning, and the psychological processes that mediate their relationship. Journal of Educational Psychology, 94(3), 545.

Alexander, P. A., Graham, S., \& Harris, K. R. (1998). A perspective on strategy research: Progress and prospects. Educational Psychology Review, 10(2), 129-154.

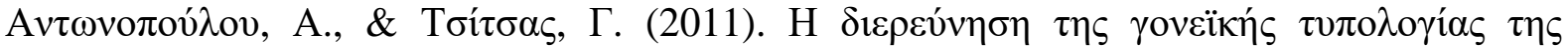

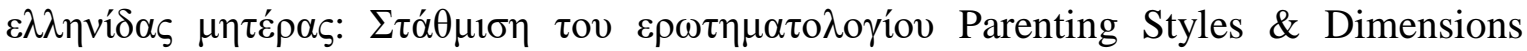

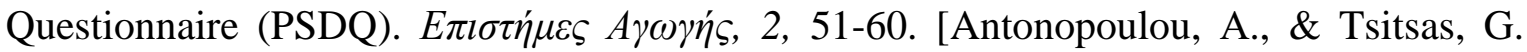
(2011). The investigation of Greek mothers' parenting styles: Adaptation of the Parenting Styles \& Dimensions Questionnaire (PSDQ). Science of Education, 2, 51-60].

Aram, D. (2002). Joint writing in Hebrew of dictated words versus proper names: Analysis of low SES mother-kindergartner dyads. Journal of Research in Childhood Education, 17(1), 47-61.

Ardelt, M., \& Eccles, J. S. (2001). Effects of mothers' parental efficacy beliefs and promotive parenting strategies on inner-city youth. Journal of Family Issues, 22(8), 944972.

Ardila, A., Rosselli, M., Matute, E., \& Guajardo, S. (2005). The influence of the parents' educational level on the development of executive functions. Developmental Neuropsychology, 28(1), 539-560.

Arnold, D. H., Zeljo, A., Doctoroff, G. L., \& Ortiz, C. (2008). Parent involvement in preschool: Predictors and the relation of involvement to preliteracy development. School Psychology Review, 37(1), 74.

Aunola, K., \& Nurmi, J. E. (2004). Maternal affection moderates the impact of psychological control on a child's mathematical performance. Developmental Psychology, 40(6), 965.

Aunola, K., Stattin, H., \& Nurmi, J. E. (2000). Parenting styles and adolescents' achievement strategies. Journal of Adolescence, 23(2), 205-222.

Bahri, A., \& Corebima, A. D. (2015). The contribution of learning motivation and metacognitive skill on cognitive learning outcome of students within different learning strategies. Journal of Baltic Science Education, 14(4). 
Bakracevic Vukman, K., \& Licardo, M. (2010). How cognitive, metacognitive, motivational and emotional self-regulation influence school performance in adolescence and early adulthood. Educational Studies, 36(3), 259-268.

Bandura, A. (1983). Temporal dynamics and decomposition of reciprocal determinism: A reply to Phillips and Orton. Psychological Review, 90(2), 166-170.

Bandura, A. (1989). Social cognitive theory. In R. Vasta (Ed.), Annals of child development. Vol. 6. Six theories of child development (pp. 1-60). Greenwich, CT: JAI Press. Retrieved from https://www.uky.edu/ eushe2/Bandura/Bandura1989ACD.pdf

Bandura, A., Barbaranelli, C., Caprara, G. V., \& Pastorelli, C. (1996). Multifaceted impact of self-efficacy beliefs on academic functioning. Child Development, 1206-1222.

Bandura, A., \& Jeffrey, R. W. (1973). Role of symbolic coding and rehearsal processes in observational learning. Journal of Personality and Social Psychology, 26(1), 122.

Barber, B. K. (1996). Parental psychological control: Revisiting a neglected construct. Child Development, 67(6), 3296-3319.

Baumrind, D. (1978). Parental disciplinary patterns and social competence in children. Youth \& Society, 9(3), 239-267.

Baumrind, D., Larzelere, R. E., \& Owens, E. B. (2010). Effects of preschool parents' power assertive patterns and practices on adolescent development. Parenting: Science and Practice, 10(3), 157-201.

Belsky, J. (1984). The determinants of parenting: A process model. Child Development, 55, 83-96.

Belsky, J., Crnic, K., \& Woodworth, S. (1995). Personality and parenting: Exploring the mediating role of transient mood and daily hassles. Journal of Personality, 63(4), 905-929.

Bembenutty, H., \& Karabenick, S. A. (1998). Academic delay of gratification. Learning and Individual Differences, 10(4), 329-346.

Berk, L. E., \& Spuhl, S. T. (1995). Maternal interaction, private speech, and task performance in preschool children. Early Childhood Research Quarterly, 10(2), 145-169.

Berlin, L. J., Brooks-Gunn, J., Spiker, D., \& Zaslow, M. J. (1995). Examining observational measures of emotional support and cognitive stimulation in black and white mothers of preschoolers. Journal of Family Issues, 16(5), 664-686.

Bernier, A., Carlson, S. M., \& Whipple, N. (2010). From external regulation to self-regulation: Early parenting precursors of young children's executive functioning. Child Development, 81(1), 326-339. 
Bidjerano, T., \& Dai, D. Y. (2007). The relationship between the big-five model of personality and self-regulated learning strategies. Learning and Individual Differences, 17(1), 69-81.

Blair, C., \& Razza, R. P. (2007). Relating effortful control, executive function, and false belief understanding to emerging math and literacy ability in kindergarten. Child Development, 78(2), 647-663.

Boekaerts, M. (1996). Self-regulated learning at the junction of cognition and motivation. European Psychologist, 1(2), 100-112.

Boekaerts, M. (1999). Self-regulated learning: Where we are today. International Journal of Educational Research, 31(6), 445-457.

Boekaerts, M., \& Corno, L. (2005). Self-regulation in the classroom: A perspective on assessment and intervention. Applied Psychology, 54(2), 199-231.

Boon, H. J. (2007). Low-and high-achieving Australian secondary school students: Their parenting, motivations and academic achievement. Australian Psychologist, 42(3), 212225.

Borkowski, J. G., Chan, L. K., \& Muthukrishna, N. (2000). 1. A process-oriented model of metacognition: Links between motivation and executive functioning. Issues in the Measurement of Metacognition, 2.

Brace, N., Snelgar, R., \& Kemp, R. (2016). SPSS for Psychologist and Everybody Else. Palgrave Macmillan.

Braungart-Rieker, J., Garwood, M. M., \& Stifter, C. A. (1997). Compliance and noncompliance: The roles of maternal control and child temperament. Journal of Applied Developmental Psychology, 18(3), 411-428.

Braveman, P. A., Cubbin, C., Egerter, S., Chideya, S., Marchi, K. S., Metzler, M., \& Carr, A., \& Pike, A. (2012). Maternal scaffolding behavior: Links with parenting style and maternal education. Developmental Psychology, 48(2), 543.

Bronson, M. (2000). Self-regulation in early childhood: Nature and nurture. New York: Guilford Press.

Bronstein, P., Ginsburg, G. S., \& Herrera, I. S. (2005). Parental predictors of motivational orientation in early adolescence: A longitudinal study. Journal of Youth and Adolescence, 34(6), 559-575.

Bryce, D., \& Whitebread, D. (2012). The development of metacognitive skills: Evidence from observational analysis of young children's behavior during problem-solving. Metacognition and Learning, 7(3), 197-217. 
Bull, R., Espy, K. A., \& Senn, T. E. (2004). A comparison of performance on the Towers of London and Hanoi in young children. Journal of Child Psychology and Psychiatry, 45(4), 743-754.

Busato, V. V., Prins, F. J., Elshout, J. J., \& Hamaker, C. (1999). The relation between learning styles, the Big Five personality traits and achievement motivation in higher education. Personality and Individual Differences, 26(1), 129-140.

Butler, D. L. (2011). Investigating self-regulated learning using in-depth case studies. In B. J. Zimmerman, \& D. H. Schunk (Eds.), Handbook of Self-Regulation of Learning and Performance (pp. 346-360). New York: Routledge.

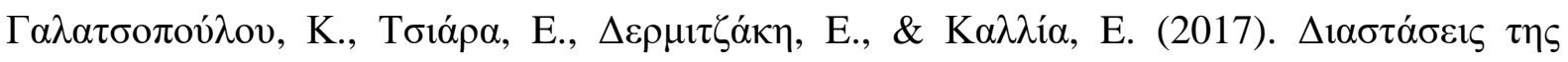

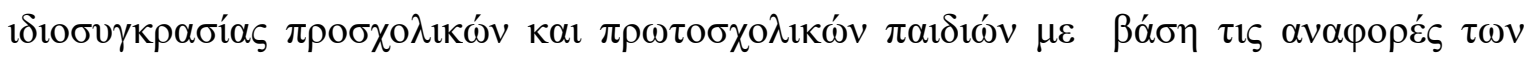

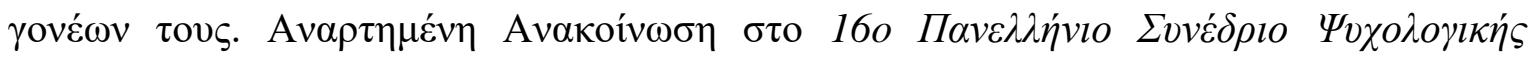

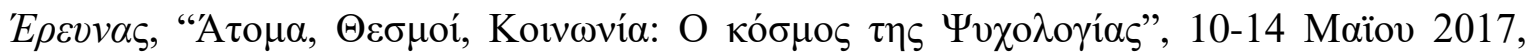

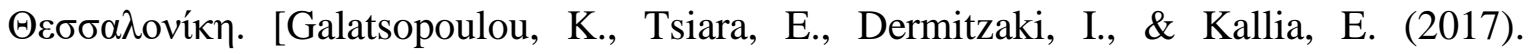
Dimensions of temperament of preschool and primary school children according to the parents' reports. Poster presentation at the $16^{\text {th }}$ Panhellenic Conference oof Psychological Research, "Individuals, Insitution, Society: The World of Phychological Research" 10-14 of May 2017, Thessaloniki].

Carr, A., \& Pike, A. (2012). Maternal scaffolding behavior: Links with parenting style and maternal education. Developmental Psychology, 48(2), 543.

Casey, B. M., Dearing, E., Dulaney, A., Heyman, M., \& Springer, R. (2014). Young girls' spatial and arithmetic performance: The mediating role of maternal supportive interactions during joint spatial problem solving. Early Childhood Research Quarterly, 29(4), 636648.

Catsambis, S. (2001). Expanding knowledge of parental involvement in children's secondary education: Connections with high school seniors' academic success. Social Psychology of Education, 5(2), 149-177.

Chaiklin, S. (2003). The zone of proximal development in Vygotsky's analysis of learning and instruction. In Kozulin, A., Gindis, B., Ageyev, V. S., \& Miller, S. M. (Eds.), Vygotsky's educational theory in cultural context (pp. 39-64). Cambridge University Press.

Chan, S. M., Bowes, J., \& Wyver, S. (2009). Parenting style as a context for emotion socialization. Early Education and Development, 20(4), 631-656.

Chatzistamatiou, M., \& Dermitzaki, I. (2013). Teaching Mathematics with self-regulation and for self-regulation: Teachers' reports. Hellenic Journal of Psychology, 10(3), 253-274. 
Chatzistamatiou, M., Dermitzaki, I., Efklides, A., \& Leondari, A. (2015). Motivational and affective determinants of self-regulatory strategy use in elementary school mathematics. Educational Psychology, 35(7), 835-850.

Cheng, N., Lu, S., Archer, M., \& Wang, Z. (2017). Quality of Maternal Parenting of 9Month-Old Infants Predicts Executive Function Performance at 2 and 3 Years of Age. Frontiers in Psychology, 8, 2293.

Cheung, C. S. S., \& Pomerantz, E. M. (2011). Parents' involvement in children's learning in the United States and China: Implications for children's academic and emotional adjustment. Child Development, 82(3), 932-950.

Cipriano, E. A., \& Stifter, C. A. (2010). Predicting preschool effortful control from toddler temperament and parenting behavior.Journal of Applied Developmental Psychology, 31(3), 221-230.

Clarke-Stewart, K. A., \& Beck, R. J. (1999). Maternal scaffolding and children's narrative retelling of a movie story. Early Childhood Research Quarterly, 14(3), 409-434.

Coleman P. K. \& Karraker K. H. (1997). Self-efficacy and parenting quality: Findings and future applications. Developmental Review 18, 47-85.

Coleman, P. K., \& Karraker, K. H. (2003). Maternal self-efficacy beliefs, competence in parenting, and toddlers' behavior and developmental status. Infant Mental Health Journal, 24(2), 126-148.

Coleman, P. K., Trent, A., Bryan, S., King, B., Rogers, N., \& Nazir, M. (2002). Parenting behavior, mothers' self-efficacy beliefs, and toddler performance on the Bayley Scales of Infant Development. Early Child Development and Care, 172(2), 123-140.

Colman, R. A., Hardy, S. A., Albert, M., Raffaelli, M., \& Crockett, L. (2006). Early predictors of self-regulation in middle childhood. Infant and Child Development, 15(4), 421-437.

Conger, R. D., Ge, X., Elder, G. H., Lorenz, F. O., \& Simons, R. L. (1994). Economic stress, coercive family process, and developmental problems of adolescents. Child Development, 65(2), 541-561.

Conner, D. B., \& Cross, D. R. (2003). Longitudinal analysis of the presence, efficacy and stability of maternal scaffolding during informal problem-solving interactions. British Journal of Developmental Psychology, 21(3), 315-334.

Considine, G., \& Zappalà, G. (2002). The influence of social and economic disadvantage in the academic performance of school students in Australia. Journal of Sociology, 38(2), $129-148$ 
Cooper, H., Lindsay, J. J., \& Nye, B. (2000). Homework in the home: How student, family, and parenting-style differences relate to the homework process. Contemporary Educational Psychology, 25(4), 464-487.

Corno, L. (1986). The metacognitive control components of self-regulated learning. Contemporary Educational Psychology, 11(4), 333-346.

Curenton, S. M., Craig, M. J., \& Flanigan, N. (2008). Use of decontextualized talk across story contexts: How oral storytelling and emergent reading can scaffold children's development. Early Education and Development, 19(1), 161-187.

Daniel, G. R., Wang, C., \& Berthelsen, D. (2016). Early school-based parent involvement, children's self-regulated learning and academic achievement: An Australian longitudinal study. Early Childhood Research Quarterly, 36, 168-177.

Darling, N., \& Steinberg, L. (1993). Parenting style as context: An integrative model. Psychological Bulletin, 113(3), 487-496.

Davis, H., \& Carr, M. (2002). Gender differences in mathematics strategy use: The influence of temperament. Learning and Individual Differences, 13(1), 83-95.

Davis-Kean, P. E. (2005). The influence of parent education and family income on child achievement: the indirect role of parental expectations and the home environment. Journal of Family Psychology, 19(2), 294.

De Haan, A. D., Prinzie, P., \& Deković, M. (2009). Mothers' and fathers' personality and parenting: The mediating role of sense of competence. Developmental Psychology, 45(6), 1695.

Dearing, E., McCartney, K., Weiss, H. B., Kreider, H., \& Simpkins, S. (2004). The promotive effects of family educational involvement for low-income children's literacy. Journal of School Psychology, 42(6), 445-460.

Deater-Deckard, K., Mullineaux, P. Y., Petrill, S. A., \& Thompson, L. A. (2009). Effortful control, surgency, and reading skills in middle childhood. Reading and Writing, 22(1), 107-116.

Deci, E. L., Driver, R. E., Hotchkiss, L., Robbins, R. J., \& Wilson, I. M. (1993). The relation of mothers' controlling vocalizations to children' $\mathrm{s}$ intrinsic motivation. Journal of Experimental Child Psychology, 55(2), 151-162.

DeFlorio, L., \& Beliakoff, A. (2015). Socioeconomic status and preschoolers' mathematical knowledge: The contribution of home activities and parent beliefs. Early Education and Development, 26(3), 319-341. 
DeGarmo, D. S., Forgatch, M. S., \& Martinez Jr, C. R. (1999). Parenting of divorced mothers as a link between social status and boys' academic outcomes: Unpacking the effects of socioeconomic status. Child Development, 70(5), 1231-1245.

Dermitzaki, I., Andreou, G., \& Paraskeva, V. (2008). High and low reading comprehension achievers' strategic behaviors and their relation to performance in a reading comprehension situation. Reading Psychology, 29(6), 471-492.

Dermitzaki, I., \& Kiosseoglou, G. (2004). Self-regulation during problem solving in secondgraders: Relations with students' performance and goal orientation. Hellenic Journal of Psychology, 1(2), 128-146.

Dermitzaki, I., \& Kriekouki, M. (2017). Implementing a teacher education program on fostering students' self-regulated learning. In D-M. Kakana, \& P. Manoli, P. (Eds.), Digital Proceedings from the 3rd International Symposium on New Issues on Teacher Education-ISNITE 2015 (pp. 302-309). Volos: University of Thessaly Press.

Dermitzaki, I., Leondari, A., \& Goudas, M. (2009). Relations between young students' strategic behaviours, domain-specific self-concept, and performance in a problem-solving situation. Learning and Instruction, 19(2), 144-157.

Devine, R. T., Bignardi, G., \& Hughes, C. (2016). Executive function mediates the relations between parental behaviors and children's early academic ability. Frontiers in Psychology, 7, 1902.

Devlin, B. (2012). The effect of maternal quality of instruction and support of development on toddler's mastery motivation. Honors Projects. Paper 158.

https://scholarworks.gvsu.edu/honorsprojects/158

Dieterich, S. E., Assel, M. A., Swank, P., Smith, K. E., \& Landry, S. H. (2006). The impact of early maternal verbal scaffolding and child language abilities on later decoding and reading comprehension skills. Journal of School Psychology, 43(6), 481-494.

Dignath, C., Buettner, G., \& Langfeldt, H. P. (2008). How can primary school students learn self-regulated learning strategies most effectively? A meta-analysis on self-regulation training programmes. Educational Research Review, 3(2), 101-129.

Dixon Jr, W. E., \& Smith, P. H. (2000). Links between early temperament and language acquisition. Merrill-Palmer Quarterly (1982-), 417-440.

Dumka, L. E., Gonzales, N. A., Wheeler, L. A., \& Millsap, R. E. (2010). Parenting selfefficacy and parenting practices over time in Mexican American families. Journal of Family Psychology, 24(5), 522. 
Duncan, G. J., \& Magnuson, K. A. (2003). Off with Hollingshead: Socioeconomic resources, parenting, and child development. In M. Bornstein \& R. Bradley (Eds.), Socioeconomic status, parenting, and child development, (pp. 83-106). Mahwah, NJ: Lawrence Erlbaum. Retrieved from http://citeseerx.ist.psu.edu/viewdoc/download?doi=10.1.1.591.6686\&rep=rep1\&type=pdf

Efklides, A. (2006). Metacognition and affect: What can metacognitive experiences tell us about the learning process? Educational Research Review, 1(1), 3-14.

Efklides, A. (2011). Interactions of metacognition with motivation and affect in self-regulated learning: The MASRL model. Educational Psychologist, 46(1), 6-25.

Eilam, B., Zeidner, M., \& Aharon, I. (2009). Student conscientiousness, self-regulated learning, and science achievement: An explorative field study. Psychology in the Schools, 46(5), 420-432.

Eisenberg, N., Fabes, R. A., Shepard, S. A., Guthrie, I. K., Murphy, B. C., \& Reiser, M. (1999). Parental reactions to children's negative emotions: Longitudinal relations to quality of children's social functioning. Child Development, 70(2), 513-534.

Eisenberg, N., Vidmar, M., Spinrad, T. L., Eggum, N. D., Edwards, A., Gaertner, B., \& Kupfer, A. (2010). Mothers' teaching strategies and children's effortful control: A longitudinal study. Developmental Psychology, 46(5), 1294.

El Nokali, N. E., Bachman, H. J., \& Votruba-Drzal, E. (2010). Parent involvement and children's academic and social development in elementary school. Child Development, 81(3), 988-1005.

Englund, M. M., Luckner, A. E., Whaley, G. J., \& Egeland, B. (2004). Children's achievement in early elementary school: Longitudinal effects of parental involvement, expectations, and quality of assistance. Journal of Educational Psychology, 96(4), 723.

Erden, M., \& Uredi, I. (2008). The effect of perceived parenting styles on self-regulated learning strategies and motivational beliefs. International Journal about Parents in Education, 2(1), 25-34.

Erdwins, C. J., Buffardi, L. C., Casper, W. J., \& O'Brien, A. S. (2001). The relationship of women's role strain to social support, role satisfaction, and self-efficacy. Family Relations, 50(3), 230-238.

Erickson, S. J., Duvall, S. W., Fuller, J., Schrader, R., MacLean, P., \& Lowe, J. R. (2013). Differential associations between maternal scaffolding and toddler emotion regulation in toddlers born preterm and full term. Early Human Development, 89(9), 699-704. 
Espy, K. A., McDiarmid, M. M., Cwik, M. F., Stalets, M. M., Hamby, A., \& Senn, T. E. (2004). The contribution of executive functions to emergent mathematic skills in preschool children. Developmental Neuropsychology, 26(1), 465-486.

Fagot, B. I., \& Gauvain, M. (1997). Mother-child problem solving: Continuity through the early childhood years. Developmental Psychology, 33(3), 480.

Fagot, B. I., \& Hagan, R. (1991). Observations of parent reactions to sex-stereotyped behaviors: Age and sex effects. Child Development, 62(3), 617-628.

Fagot, B. I., \& Kavanagh, K. (1993). Parenting during the second year: Effects of children's age, sex, and attachment classification. Child Development, 64(1), 258-271.

Fan, W., \& Williams, C. M. (2010). The effects of parental involvement on students' academic self-efficacy, engagement and intrinsic motivation. Educational Psychology, $30(1), 53-74$.

Fan, X., \& Chen, M. (2001). Parental involvement and students' academic achievement: A meta-analysis. Educational Psychology Review, 13(1), 1-22.

Fantuzzo, J., McWayne, C., Perry, M. A., \& Childs, S. (2004). Multiple dimensions of family involvement and their relations to behavioral and learning competencies for urban, lowincome children. School Psychology Review, 33(4), 467.

Feldman, R., Greenbaum, C. W., Mayes, L. C., \& Erlich, S. H. (1997). Change in motherinfant interactive behavior: Relations to change in the mother, the infant, and the social context. Infant Behavior and Development, 20(2), 151-163.

Fender, J. G., Richert, R. A., Robb, M. B., \& Wartella, E. (2010). Parent teaching focus and toddlers' learning from an infant DVD. Infant and Child Development, 19(6), 613-627.

Field, A. (2013). Discovering statistics using IBM SPSS statistics. London: Sage.

Forehand, R., \& Jones, D. J. (2002). The stability of parenting: A longitudinal analysis of inner-city African-American mothers. Journal of Child and Family Studies, 11(4), 455467.

Foster, M. A., Lambert, R., Abbott-Shim, M., McCarty, F., \& Franze, S. (2005). A model of home learning environment and social risk factors in relation to children's emergent literacy and social outcomes. Early Childhood Research Quarterly, 20(1), 13-36.

Frankel, R. (2016). After the test: What now? Post assessment reflection. AURCO Journal, 22, (82-93).

Fuhs, M. W., Nesbitt, K. T., Farran, D. C., \& Dong, N. (2014). Longitudinal associations between executive functioning and academic skills across content areas. Developmental Psychology, 50(6), 1698. 
Garello, V., Viterbori, P., \& Usai, M. C. (2012). Temperamental profiles and language development: A replication and an extension. Infant Behavior and Development, 35(1), 71-82.

Gartstein, M. A., \& Rothbart, M. K. (2003). Studying infant temperament via the revised Infant Behavior Questionnaire. Infant Behavior and Development, 26(1), 64-86.

Gauvain, M., \& Fagot, B. (1995). Child Temperament as a mediator of mother-toddler problem solving. Social Development, 4(3), 257-276.

Gauvain, M., \& Perez, S. M. (2008). Mother-child planning and child compliance. Child Development, 79(3), 761-775.

Ghera, M. M., Hane, A. A., Malesa, E. E., \& Fox, N. A. (2006). The role of infant soothability in the relation between infant negativity and maternal sensitivity. Infant Behavior and Development, 29(2), 289-293.

Giallo, R., Treyvaud, K., Cooklin, A., \& Wade, C. (2013). Mothers' and fathers' involvement in home activities with their children: Psychosocial factors and the role of parental selfefficacy. Early Child Development and Care, 183(3-4), 343-359.

Ginsburg, G. S., \& Bronstein, P. (1993). Family factors related to children's intrinsic/extrinsic motivational orientation and academic performance. Child Development, 64(5), 14611474.

Glatz, T., \& Buchanan, C. M. (2015). Over-time associations among parental self-efficacy, promotive parenting practices, and adolescents' externalizing behaviors. Journal of Family Psychology, 29(3), 427.

Goldsmith, H. H., Buss, A. H., Plomin, R., Rothbart, M. K., Thomas, A., Chess, S., ... \& McCall, R. B. (1987). Roundtable: What is temperament? Four approaches. Child Development, 58(5), 529.

Gonida, E. N., \& Cortina, K. S. (2014). Parental involvement in homework: Relations with parent and student achievement-related motivational beliefs and achievement. British Journal of Educational Psychology, 84(3), 376-396.

Gonzalez, A. L., \& Wolters, C. A. (2006). The relation between perceived parenting practices and achievement motivation in mathematics. Journal of Research in Childhood Education, 21(2), 203-217.

González, M. M. (1996). Tasks and activities. A parent-child interaction analysis. Learning and Instruction, 6(4), 287-306. 
Gonzalez-DeHass, A. R., Willems, P. P., \& Holbein, M. F. D. (2005). Examining the relationship between parental involvement and student motivation. Educational Psychology Review, 17(2), 99-123.

Gottfried, A. E., Fleming, J. S., \& Gottfried, A. W. (1994). Role of parental motivational practices in children's academic intrinsic motivation and achievement. Journal of Educational Psychology, 86(1), 104.

Grolnick, W. S., \& Slowiaczek, M. L. (1994). Parents' involvement in children's schooling: A multidimensional conceptualization and motivational model. Child Development, 65(1), 237-252.

Grolnick, W. S., Friendly, R. W., \& Bellas, V. M. (2009). Parenting and children's motivation at school. In Wentzel, K. R., \& Miele, D. B. (Eds.), Handbook of motivation at school (pp. 279-300). New York, NY, US: Routledge.

Grolnick, W. S., Gurland, S. T., DeCourcey, W., \& Jacob, K. (2002). Antecedents and consequences of mothers' autonomy support: An experimental investigation. Developmental Psychology, 38(1), 143.

Grolnick, W. S., Kurowski, C. O., Dunlap, K. G., \& Hevey, C. (2000). Parental resources and the transition to junior high. Journal of Research on Adolescence, 10(4), 465-488.

Gurland, S. T., \& Grolnick, W. S. (2005). Perceived threat, controlling parenting, and children's achievement orientations. Motivation and Emotion, 29(2), 103-121.

Halpern, L. F., \& Mclean Jr, W. E. (1997). Hey mom, look at me! Infant Behavior and Development, 20(4), 515-529.

Hammond, J., \& Gibbons, P. (2005). What is scaffolding. Teachers' Voices, 8, 8-16.

Hammond, S. I., Müller, U., Carpendale, J. I., Bibok, M. B., \& Liebermann-Finestone, D. P. (2012). The effects of parental scaffolding on preschoolers' executive function. Developmental Psychology, 48(1), 271.

Harris, A., \& Goodall, J. (2008). Do parents know they matter? Engaging all parents in learning. Educational Research, 50(3), 277-289.

Haslam, D. M., Pakenham, K. I., \& Smith, A. (2006). Social support and postpartum depressive symptomatology: The mediating role of maternal self-efficacy. Infant Mental Health Journal, 27(3), 276-291.

Hayes, A. F. (2018). Introduction to mediation, moderation, and conditional process analysis: A regression-based approach. New York: Guilford Publications. 
Hess, C. R., Teti, D. M., \& Hussey-Gardner, B. (2004). Self-efficacy and parenting of highrisk infants: The moderating role of parent knowledge of infant development. Journal of Applied Developmental Psychology, 25(4), 423-437.

Hidi, S., \& Renninger, K. A. (2006). The four-phase model of interest development. Educational Psychologist, 41(2), 111-127.

Hill, N. E., \& Bush, K. R. (2001). Relationships between parenting environment and children's mental health among African American and European American mothers and children. Journal of Marriage and Family, 63(4), 954-966.

Hill, N. E., \& Tyson, D. F. (2009). Parental involvement in middle school: A meta-analytic assessment of the strategies that promote achievement. Developmental Psychology, 45(3), 740.

Hoff, E. (2003). The specificity of environmental influence: Socioeconomic status affects early vocabulary development via maternal speech. Child Development, 74(5), 1368-1378.

Hoff, E., \& Tian, C. (2005). Socioeconomic status and cultural influences on language. Journal of Communication Disorders, 38(4), 271-278.

Hoff-Ginsberg, E. (1991). Mother-child conversation in different social classes and communicative settings. Child Development, 62(4), 782-796.

Hokoda, A., \& Fincham, F. D. (1995). Origins of children's helpless and mastery achievement patterns in the family. Journal of Educational Psychology, 87(3), 375.

Hong, E., Peng, Y., \& Rowell, L. L. (2009). Homework self-regulation: Grade, gender, and achievement-level differences. Learning and Individual Differences, 19(2), 269-276.

Hoover-Dempsey, K. V., Bassler, O. C., \& Brissie, J. S. (1992). Explorations in parentschool relations. The Journal of Educational Research, 85(5), 287-294.

Hoover-Dempsey, K. V., Battiato, A. C., Walker, J. M., Reed, R. P., DeJong, J. M., \& Jones, K. P. (2001). Parental involvement in homework. Educational Psychologist, 36(3), 195209.

Hoover-Dempsey, K. V., \& Sandler, H. M. (1995). Parental involvement in children's education: Why does it make a difference? Teachers College Record, 95, 310-331.

Hubbs-Tait, L., Culp, A. M., Culp, R. E., \& Miller, C. E. (2002). Relation of maternal cognitive stimulation, emotional support, and intrusive behavior during Head Start to children's kindergarten cognitive abilities. Child Development, 73(1), 110-131.

Hustedt, J. T., \& Raver, C. C. (2002). Scaffolding in low-income mother-child dyads: Relations with joint attention and dyadic reciprocity. International Journal of Behavioral Development, 26(2), 113-119. 
Järvelä, S., Järvenoja, H., \& Malmberg, J. (2012). How elementary school students' motivation is connected to self-regulation. Educational Research and Evaluation, 18(1), 65-84.

Johnson, V. K., Cowan, P. A., \& Cowan, C. P. (1999). Children's classroom behavior: The unique contribution of family organization. Journal of Family Psychology, 13(3), 355.

Joussemet, M., Koestner, R., Lekes, N., \& Landry, R. (2005). A longitudinal study of the relationship of maternal autonomy support to children's adjustment and achievement in school. Journal of Personality, 73(5), 1215-1236.

Jusiene, R., Breidokiene, R., \& Pakalniskiene, V. (2015). Developmental trajectories of mother reported regulatory problems from toddlerhood to preschool age. Infant Behavior and Development, 40, 84-94.

Kafoussi, S. (2009). Parents' and students' interaction in mathematics: Designing home mathematical activities. In Proceedings of CIEAEM 58 - SRNI, Czech Republic, 209-214.

Kannass, K. N., Oakes, L. M., \& Shaddy, D. J. (2006). A longitudinal investigation of the development of attention and distractibility. Journal of Cognition and Development, 7(3), 381-409.

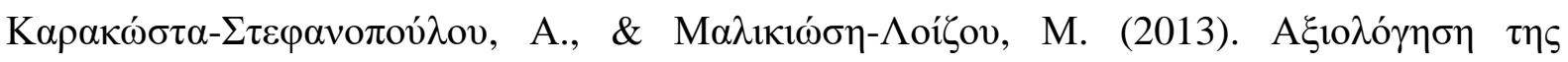

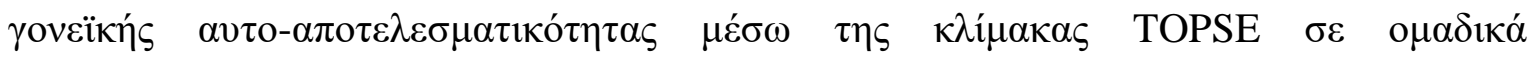

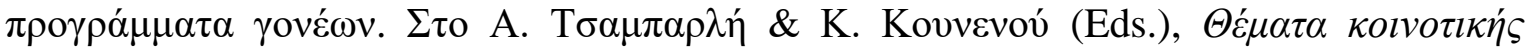

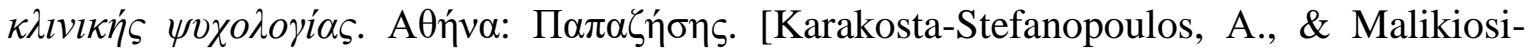
Loizos, M. (2013). Evaluation of parental self-efficacy using the TOPSE scale in group parent education programs. In A. Tsamparli \& K. Kounenou (Eds.), Issues of Community Clinical Psychology (in Greek). Athens: Papazisis]. Retrieved from: https://www.researchgate.net/publication/266394616.

Karavasilis, L., Doyle, A. B., \& Markiewicz, D. (2003). Associations between parenting style and attachment to mother in middle childhood and adolescence. International Journal of Behavioral Development, 27(2), 153-164.

Karbach, J., Gottschling, J., Spengler, M., Hegewald, K., \& Spinath, F. M. (2013). Parental involvement and general cognitive ability as predictors of domain-specific academic achievement in early adolescence. Learning and Instruction, 23, 43-51.

Karreman, A., Van Tuijl, C., Van Aken, M. A., \& Deković, M. (2008). Parenting, coparenting, and effortful control in preschoolers. Journal of Family Psychology, 22(1), 30. 
Kendall, S. \& Bloomfield, L. (2005). Developing and validating a tool to measure parenting self-efficacy. Journal of Advanced Nursing, 51(2), 174-181

Kermani, H., \& Brenner, M. E. (2000). Maternal scaffolding in the child's zone of proximal development across tasks: Cross-cultural perspectives. Journal of Research in Childhood Education, 15(1), 30-52.

Kermani, H., \& Janes, H. A. (1999). Adjustment across task in maternal scaffolding in lowincome Latino immigrant families. Hispanic Journal of Behavioral Sciences, 21(2), 134153.

Knollmann, M., \& Wild, E. (2007). Quality of parental support and students' emotions during homework: Moderating effects of students' motivational orientations. European Journal of Psychology of Education, 22(1), 63.

Kochanska, G., Murray, K. T., \& Harlan, E. T. (2000). Effortful control in early childhood: continuity and change, antecedents, and implications for social development. Developmental Psychology, 36(2), 220.

Komarraju, M., Karau, S. J., Schmeck, R. R., \& Avdic, A. (2011). The Big Five personality traits, learning styles, and academic achievement. Personality and Individual Differences, 51(4), 472-477.

Kolb, B., \& Gibb, R. (2011). Brain plasticity and behaviour in the developing brain. Journal of the Canadian Academy of Child and Adolescent Psychiatry, 20(4), 265.

Korat, O. (2009). The effect of maternal teaching talk on children's emergent literacy as a function of type of activity and maternal education level. Journal of Applied Developmental Psychology, 30(1), 34-42.

Koutsoulis, M. K., \& Campbell, J. R. (2001). Family processes affect students' motivation, and science and math achievement in Cypriot high schools. Structural Equation Modeling, $8(1), 108-127$.

Kramer, K. Z. (2012). Parental behavioural control and academic achievement: striking the balance between control and involvement. Research in Education, 88(1), 85-98.

Laake, L. M., \& Bridgett, D. J. (2014). Happy babies, chatty toddlers: Infant positive affect facilitates early expressive, but not receptive language. Infant Behavior and Development, 37(1), 29-32.

Lamborn, S. D., Mounts, N. S., Steinberg, L., \& Dornbusch, S. M. (1991). Patterns of competence and adjustment among adolescents from authoritative, authoritarian, indulgent, and neglectful families. Child Development, 62(5), 1049-1065. 
Landry, S. H., Miller-Loncar, C. L., Smith, K. E., \& Swank, P. R. (2002). The role of early parenting in children's development of executive processes. Developmental Neuropsychology, 21(1), 15-41.

Landry, S. H., Smith, K. E., \& Swank, P. R. (2006). Responsive parenting: Establishing early foundations for social, communication, and independent problem-solving skills. Developmental Psychology, 42(4), 627.

Leerkes, E. M., Blankson, A. N., O'Brien, M., Calkins, S. D., \& Marcovitch, S. (2011). The relation of maternal emotional and cognitive support during problem solving to pre-academic skills in preschoolers. Infant and Child Development, 20(6), 353-370.

Leerkes, E. M., \& Crockenberg, S. C. (2002). The development of maternal self-efficacy and its impact on maternal behavior. Infancy, 3(2), 227-247.

Lengua, L. J. (2006). Growth in temperament and parenting as predictors of adjustment during children's transition to adolescence. Developmental Psychology, 42(5), 819.

Lengua, L. J., Honorado, E., \& Bush, N. R. (2007). Contextual risk and parenting as predictors of effortful control and social competence in preschool children. Journal of Applied Developmental Psychology, 28(1), 40-55.

Lengua, L. J., \& Kovacs, E. A. (2005). Bidirectional associations between temperament and parenting and the prediction of adjustment problems in middle childhood. Journal of Applied Developmental Psychology, 26(1), 21-38.

Leung, P. W., \& Kwan, K. S. (1998). Parenting styles, motivational orientations, and selfperceived academic competence: A mediational model. Merrill-Palmer Quarterly (1982-), $1-19$.

Leve, L. D., \& Fagot, B. I. (1997). Prediction of positive peer relations from observed parent-child interactions. Social Development, 6(2), 254-269.

Liew, J., McTigue, E. M., Barrois, L., \& Hughes, J. N. (2008). Adaptive and effortful control and academic self-efficacy beliefs on achievement: A longitudinal study of 1st through 3rd graders. Early Childhood Research Quarterly, 23(4), 515-526.

Loeber, R., Drinkwater, M., Yin, Y., Anderson, S. J., Schmidt, L. C., \& Crawford, A. (2000). Stability of family interaction from ages 6 to 18. Journal of Abnormal Child Psychology, 28(4), 353-369.

Losoya, S. H., Callor, S., Rowe, D. C., \& Goldsmith, H. H. (1997). Origins of familial similarity in parenting: A study of twins and adoptive siblings. Developmental Psychology, 33(6), 1012. 
Lowe, J., Erickson, S. J., MacLean, P., Duvall, S. W., Ohls, R. K., \& Duncan, A. F. (2014). Associations between maternal scaffolding and executive functioning in 3 and 4year olds born very low birth weight and normal birth weight. Early Human Development, 90(10), 587-593.

Lowe, J. R., Erickson, S. J., MacLean, P., Schrader, R., \& Fuller, J. (2013). Association of maternal scaffolding to maternal education and cognition in toddlers born preterm and full term. Acta Paediatrica, 102(1), 72-77.

Lunkenheimer, E. S., Dishion, T. J., Shaw, D. S., Connell, A. M., Gardner, F., Wilson, M. N., \& Skuban, E. M. (2008). Collateral benefits of the family check-up on early childhood school readiness: Indirect effects of parents' positive behavior support. Developmental Psychology, 44(6), 1737.

Lynch, J. (2002). Parents' self-efficacy beliefs, parents' gender, children's reader self-perceptions, reading achievement and gender. Journal of Research in Reading, 25(1), 54-67.

Magnuson, K. A., \& Duncan, G. J. (2006). The role of family socioeconomic resources in the black-white test score gap among young children. Developmental Review, 26(4), 365-399.

Mariani, L. (1997). Teacher support and teacher challenge in promoting learner autonomy. Perspectives: A Journal of TESOL Italy, XXIII (2). Retrieved from http://www.learningpaths.org/papers/papersupport.htm

Martin, L. E., \& Reutzel, D. R. (1996). Scaffolding books for children: Mothers' metacognitive decisions. Reading Psychology: An International Quarterly, 17(2), 159180.

Maruno, S. I., \& Zhang, L. (2010). Causal relationships among academic delay of gratification, motivation, and self-regulated learning in elementary school children. Perceptual and Motor Skills, 111(2), 631-642.

Mattanah, J. F., Pratt, M. W., Cowan, P. A., \& Cowan, C. P. (2005). Authoritative parenting, parental scaffolding of long-division mathematics, and children's academic competence in fourth grade. Journal of Applied Developmental Psychology, 26(1), 85-106.

Mayer, R. E. (1998). Cognitive, metacognitive, and motivational aspects of problem solving. Instructional Science, 26(1-2), 49-63.

McClelland, M. M., Acock, A. C., \& Morrison, F. J. (2006). The impact of kindergarten learning-related skills on academic trajectories at the end of elementary school. Early Childhood Research Quarterly, 21(4), 471-490. 
McClelland, M. M., Cameron, C. E., Connor, C. M., Farris, C. L., Jewkes, A. M., \& Morrison, F. J. (2007). Links between behavioral regulation and preschoolers' literacy, vocabulary, and math skills. Developmental Psychology, 43(4), 947.

McNally, S., Eisenberg, N., \& Harris, J. D. (1991). Consistency and change in maternal child-rearing practices and values: A longitudinal study. Child Development, 62(1), 190198.

Meins, E., Fernyhough, C., Fradley, E., \& Tuckey, M. (2001). Rethinking maternal sensitivity: Mothers' comments on infants' mental processes predict security of attachment at 12 months. The Journal of Child Psychology and Psychiatry and Allied Disciplines, 42(5), 637-648.

Mermelshtine, R., \& Barnes, J. (2016). Maternal responsive-didactic caregiving in play interactions with 10-month-olds and cognitive development at 18 months. Infant and Child Development, 25(3), 296-316.

Merz, E. C., Landry, S. H., Montroy, J. J., \& Williams, J. M. (2017). Bidirectional associations between parental responsiveness and executive function during early childhood. Social Development, 26(3), 591-609.

Mih, V. (2013). Role of parental support for learning, autonomous/control motivation, and forms of self-regulation on academic attainment in high school students: A path analysis. Cognition, Brain, Behavior, 17(1), 35.

Mills-Koonce, W. R., Gariépy, J. L., Propper, C., Sutton, K., Calkins, S., Moore, G., \& Cox, M. (2007). Infant and parent factors associated with early maternal sensitivity: A caregiver-attachment systems approach. Infant Behavior and Development, 30(1), 114126.

Mokrova, I. L., O'Brien, M., Calkins, S. D., Leerkes, E. M., \& Marcovitch, S. (2012). Links between family social status and preschoolers' persistence: The role of maternal values and quality of parenting. Infant and Child Development, 21(6), 617-633.

Mol, S. E., \& Neuman, S. B. (2014). Sharing information books with kindergartners: The role of parents' extra-textual talk and socioeconomic status. Early Childhood Research Quarterly, 29(4), 399-410.

Morales, M., Mundy, P., Delgado, C. E., Yale, M., Neal, R., \& Schwartz, H. K. (2000). Gaze following, temperament, and language development in 6-month-olds: A replication and extension. Infant Behavior and Development, 23(2), 231-236.

Morrongiello, B. A., Corbett, M., Lasenby, J., Johnston, N., \& McCourt, M. (2006). Factors influencing young children's risk of unintentional injury: Parenting style and strategies for 
teaching about home safety. Journal of Applied Developmental Psychology, 27(6), 560570.

Moss, E., \& Strayer, F. F. (1990). Interactive problem-solving of gifted and non-gifted preschoolers with their mothers. International Journal of Behavioral Development, 13(2), 177-197.

Mulvaney, M. K., McCartney, K., Bub, K. L., \& Marshall, N. L. (2006). Determinants of dyadic scaffolding and cognitive outcomes in first graders. Parenting: Science and Practice, 6(4), 297-320.

Murdock, K. W. (2013). An examination of parental self-efficacy among mothers and fathers. Psychology of Men \& Masculinity, 14(3), 314.

Natsiopoulou, T., Souliotis, D., Kyridis, A., \& Hatzisavvides, S. (2000). Reading children's books to the preschool children in Greek families. International Journal of Early Childhood, 38(2), 69-79.

Neitzel, C., \& Stright, A. D. (2003). Mothers' scaffolding of children's problem solving: establishing a foundation of academic self-regulatory competence. Journal of Family Psychology, 17(1), 147.

Neitzel, C., \& Stright, A. D. (2004). Parenting behaviours during child problem solving: The roles of child temperament, mother education and personality, and the problem-solving context. International Journal of Behavioral Development, 28(2), 166-179.

Noble, K. G., Norman, M. F., \& Farah, M. J. (2005). Neurocognitive correlates of socioeconomic status in kindergarten children. Developmental Science, 8(1), 74-87.

Noel, M., Peterson, C., \& Jesso, B. (2008). The relationship of parenting stress and child temperament to language development among economically disadvantaged preschoolers. Journal of Child Language, 35(4), 823-843.

Nurmi, J. E., \& Aunola, K. (2005). Task-motivation during the first school years: A personoriented approach to longitudinal data. Learning and Instruction, 15(2), 103-122.

Obradović, J., Yousafzai, A. K., Finch, J. E., \& Rasheed, M. A. (2016). Maternal scaffolding and home stimulation: Key mediators of early intervention effects on children's cognitive development. Developmental Psychology, 52(9), 1409.

Pajares, F., \& Valiante, G. (2002). Students'self-efficacy in their self-regulated learning strategies: A developmental perspective. Psychologia, 45(4), 211-221.

Pappas, S., Ginsburg, H. P., \& Jiang, M. (2003). SES differences in young children's metacognition in the context of mathematical problem solving. Cognitive Development, 18(3), 431-450. 
Paris, S. G., \& Paris, A. H. (2001). Classroom applications of research on self-regulated learning. Educational Psychologist, 36(2), 89-101

Patall, E. A., Cooper, H., \& Robinson, J. C. (2008). Parent involvement in homework: A research synthesis. Review of Educational Research, 78(4), 1039-1101.

Pea, R. D. (2004). The social and technological dimensions of scaffolding and related theoretical concepts for learning, education, and human activity. The Journal of the Learning Sciences, 13(3), 423-451

Pellegrini, A. D., McGillicuddy-DeLisi, A. V., Sigel, I. E., \& Brody, G. H. (1986). The effects of children's communicative status and task on parents' teaching strategies. Contemporary Educational Psychology, 11(3), 240-252.

Pelletier, J., \& Brent, J. M. (2002). Parent participation in children's school readiness: The effects of parental self-efficacy, cultural diversity and teacher strategies. International Journal of Early Childhood, 34(1), 45-60.

Petrogiannis, K. (2013). Early childhood education and care in Greece: In search of an identity. Tidsskrift for Nordisk Barnehageforskning, 6 (29), 1-9.

Pianta, R. C., Nimetz, S. L., \& Bennett, E. (1997). Mother-child relationships, teacher-child relationships, and school outcomes in preschool and kindergarten. Early Childhood Research Quarterly, 12(3), 263-280.

Pino-Pasternak, D., \& Whitebread, D. (2010). The role of parenting in children's selfregulated learning. Educational Research Review, 5(3), 220-242.

Pino-Pasternak, D., Whitebread, D., \& Tolmie, A. (2010). A multidimensional analysis of parent-child interactions during academic tasks and their relationships with children's selfregulated learning. Cognition and Instruction, 28(3), 219-272.

Pintrich, P. R. (1995). Understanding self-regulated learning. New Directions for Teaching and Learning, 1995(63), 3-12.

Pintrich, P. R. (2000). The role of goal orientation in learning and achievement. In M. Boekaerts, P. R. Pintrich, \& M. Zeidner (Eds.), Handbook of self-regulation (pp. 451-502). San Diego: Academic Press.

Pintrich, P. R. (2004). A conceptual framework for assessing motivation and self-regulated learning in college students. Educational Psychology Review, 16(4), 385-407.

Pintrich, P. R., \& De Groot, E. V. (1990). Motivational and self-regulated learning components of classroom academic performance. Journal of Educational Psychology, $82(1), 33$. 
Pintrich, P. R., Smith, D. A. F., Duncan, T., \& Mckeachie, W. J. (1991). A Manual for the Use of the Motivated Strategies for Learning Questionnaire (MSLQ). Retrieved from https://files.eric.ed.gov/fulltext/ED338122.pdf

Plumert, J. M., \& Nichols-Whitehead, P. (1996). Parental scaffolding of young children's spatial communication. Developmental Psychology, 32(3), 523.

Pomerantz, E. M., \& Eaton, M. M. (2001). Maternal intrusive support in the academic context: transactional socialization processes. Developmental Psychology, 37(2), 174.

Porter, C. L., \& Hsu, H. C. (2003). First-time mothers' perceptions of efficacy during the transition to motherhood: Links to infant temperament. Journal of Family Psychology, 17(1), 54.

Porter, C. L., Hart, C. H., Yang, C., Robinson, C. C., Olsen, S. F., Zeng, Q., ... \& Jin, S. (2005). A comparative study of child temperament and parenting in Beijing, China and the western United States. International Journal of Behavioral Development, 29(6), 541-551.

Pratt, M. W., Green, D., MacVicar, J., \& Bountrogianni, M. (1992). The mathematical parent: Parental scaffolding, parenting style, and learning outcomes in long-division mathematics homework. Journal of Applied Developmental Psychology, 13(1), 17-34.

Pratt, M. W., Kerig, P., Cowan, P. A., \& Cowan, C. P. (1988). Mothers and fathers teaching 3-year-olds: Authoritative parenting and adult scaffolding of young children's learning. Developmental Psychology, 24(6), 832.

Pratt, M. W., Kerig, P. K., Cowan, P. A., \& Cowan, C. P. (1992). Family worlds: Couple satisfaction, parenting style, and mothers' and fathers' speech to young children. MerrillPalmer Quarterly (1982-), 245-262.

Puklek Levpušček, M., \& Zupančič, M. (2009). Math achievement in early adolescence: The role of parental involvement, teachers' behavior, and students' motivational beliefs about math. The Journal of Early Adolescence, 29(4), 541-570.

Puntambekar, S., \& Hübscher, R. (2005). Tools for scaffolding students in a complex learning environment: What have we gained and what have we missed? Educational Psychologist, 40(1), 1-12.

Purdie, N., Carroll, A., \& Roche, L. (2004). Parenting and adolescent self-regulation. Journal of Adolescence, 27(6), 663-676.

Putnam, S. P., \& Rothbart, M. K. (2006). Development of short and very short forms of the Children's Behavior Questionnaire. Journal of Personality Assessment, 87(1), 102-112.

Puustinen, M., Lyyra, A. L., Metsäpelto, R. L., \& Pulkkinen, L. (2008). Children's help seeking: The role of parenting. Learning and Instruction, 18(2), 160-171. 
Ratner, H. H., \& Stettner, L. J. (1991). Thinking and feeling: Putting Humpty Dumpty together again. Merrill-Palmer Quarterly (1982-), 1-26.

Raviv, T., Kessenich, M., \& Morrison, F. J. (2004). A mediational model of the association between socioeconomic status and three-year-old language abilities: The role of parenting factors. Early Childhood Research Quarterly, 19(4), 528-547.

Razza, R. A., \& Raymond, K. (2013). Associations among maternal behavior, delay of gratification, and school readiness across the early childhood years. Social Development, 22(1), 180-196.

Rhoades, B. L., Greenberg, M. T., Lanza, S. T., \& Blair, C. (2011). Demographic and familial predictors of early executive function development: Contribution of a personcentered perspective. Journal of Experimental Child Psychology, 108(3), 638-662.

Richman, E. R., \& Rescorla, L. (1995). Academic orientation and warmth in mothers and fathers of preschoolers: Effects on academic skills and self-perceptions of competence. Early Education and Development, 6(3), 197-213.

Rimm-Kaufman, S. E., Curby, T. W., Grimm, K. J., Nathanson, L., \& Brock, L. L. (2009). The contribution of children's self-regulation and classroom quality to children's adaptive behaviors in the kindergarten classroom. Developmental Psychology, 45(4), 958.

Roberts, E., Bornstein, M. H., Slater, A. M., \& Barrett, J. (1999). Early cognitive development and parental education. Infant and Child Development, 8(1), 49-62.

Robinson, J. B., Burns, B. M., \& Davis, D. W. (2009). Maternal scaffolding and attention regulation in children living in poverty. Journal of Applied Developmental Psychology, 30(2), 82-91.

Robinson, C. C., Mandleco, B., Olsen, S. F., \& Hart, C. H. (2001). The Parenting Styles and Dimensions Questionnaire (PSDQ). Handbook of Family Measurement Techniques, 3, 319-321.

Rogers, M. A., Theule, J., Ryan, B. A., Adams, G. R., \& Keating, L. (2009). Parental involvement and children's school achievement: Evidence for mediating processes. Canadian Journal of School Psychology, 24(1), 34-57.

Rogoff, B., Ellis, S., \& Gardner, W. (1984). Adjustment of adult-child instruction according to child's age and task. Developmental Psychology, 20(2), 193.

Rotgans, J., \& Schmidt, H. (2009). Examination of the context-specific nature of self-regulated learning. Educational Studies, 35(3), 239-253. 
Rothbart, M. K., Ahadi, S. A., Hershey, K. L., \& Fisher, P. (2001). Investigations of temperament at three to seven years: The Children's Behavior Questionnaire. Child Development, 72(5), 1394-1408.

Rothbart, M. K., Ellis, L. K., \& Posner, M. I. (2004). Temperament and selfregulation. Handbook of Self-regulation: Research, Theory, and Applications, 2, 441-460.

Rothbart, M. K., Posner, M. I., \& Hershey, K. L. (2006). Temperament, attention, and developmental psychopathology. Developmental Psychopathology, 2, 465-501.

Rowe, M. L. (2008). Child-directed speech: relation to socioeconomic status, knowledge of child development and child vocabulary skill. Journal of Child Language, 35(1), 185-205.

Rudy, D., \& Grusec, J. E. (2006). Authoritarian parenting in individualist and collectivist groups: Associations with maternal emotion and cognition and children's selfesteem. Journal of Family Psychology, 20(1), 68.

Salonen, P., Lepola, J., \& Vauras, M. (2007). Scaffolding interaction in parent-child dyads: Multimodal analysis of parental scaffolding with task and non-task oriented children. European Journal of Psychology of Education, 22(1), 77-96.

Sarsour, K., Sheridan, M., Jutte, D., Nuru-Jeter, A., Hinshaw, S., \& Boyce, W. T. (2011). Family socioeconomic status and child executive functions: The roles of language, home environment, and single parenthood. Journal of the International Neuropsychological Society, 17(01), 120-132.

Scaramella, L. V., Sohr-Preston, S. L., Mirabile, S. P., Robison, S. D., \& Callahan, K. L. (2008). Parenting and children's distress reactivity during toddlerhood: An examination of direction of effects. Social Development, 17(3), 578-595.

Schiefele, U., \& Csikszentmihalyi, M. (1995). Motivation and ability as factors in mathematics experience and achievement. Journal for Research in Mathematics Education, 163-181.

Schraw, G. (1998). Promoting general metacognitive awareness. Instructional Science, 26(12), 113-125.

Schneider, W. (2008). The development of metacognitive knowledge in children and adolescents: Major trends and implications for education. Mind, Brain, and Education, 2(3), 114-121.

Schunk, D. H. (2012) (6 $6^{\text {th }}$ edition). Constructivism. In Learning theories. An educational perspective (pp. 228-277). Boston: Pearson.

Schunk, D. H. (2012) $\left(6^{\text {th }}\right.$ edition). Social cognitive theory. In Learning theories. An educational perspective (pp. 117-162). Boston: Pearson. 
Schunk, D. H., \& Zimmerman, B. J. (1997). Social origins of self-regulatory competence. Educational Psychologist, 32(4), 195-208.

Schunk, D. H., \& Zimmerman, B. J. (2008). Motivation and Self-Regulated Learning. Theory, Research and Application. New York: Lawrence Erlbaum Associates.

Shumow, L., \& Lomax, R. (2002). Parental efficacy: Predictor of parenting behavior and adolescent outcomes. Parenting: Science and Practice, 2(2), 127-150.

Shumow, L., Vandell, D. L., \& Posner, J. K. (1998). Harsh, firm, and permissive parenting in low-income families: Relations to children's academic achievement and behavioral adjustment. Journal of Family Issues, 19(5), 483-507.

Silinskas, G., Kiuru, N., Aunola, K., Lerkkanen, M. K., \& Nurmi, J. E. (2015). The developmental dynamics of children's academic performance and mothers' homeworkrelated affect and practices. Developmental Psychology, 51(4), 419.

Skibbe, L., Behnke, M., \& Justice, L. M. (2004). Parental scaffolding of children's phonological awareness skills: Interactions between mothers and their preschoolers with language difficulties. Communication Disorders Quarterly, 25(4), 189-203.

Slagt, M., Deković, M., de Haan, A. D., van den Akker, A. L., \& Prinzie, P. (2012). Longitudinal associations between mothers' and fathers' sense of competence and children's externalizing problems: The mediating role of parenting. Developmental Psychology, 48(6), 1554.

Smith, K. E., Landry, S. H., \& Swank, P. R. (2000). Does the content of mothers' verbal stimulation explain differences in children's development of verbal and nonverbal cognitive skills? Journal of School Psychology, 38(1), 27-49.

Spinrad, T. L., Eisenberg, N., Gaertner, B., Popp, T., Smith, C. L., Kupfer, A., ... \& Hofer, C. (2007). Relations of maternal socialization and toddlers' effortful control to children's adjustment and social competence. Developmental Psychology, 43(5), 1170.

Spinrad, T. L., Eisenberg, N., Silva, K. M., Eggum, N. D., Reiser, M., Edwards, A., ... \& Hayashi, A. (2012). Longitudinal relations among maternal behaviors, effortful control and young children's committed compliance. Developmental Psychology, 48(2), 552.

Steca, P., Bassi, M., Caprara, G. V., \& Delle Fave, A. (2011). Parents' self-efficacy beliefs and their children's psychosocial adaptation during adolescence. Journal of Youth and Adolescence, 40(3), 320-331.

Steinberg, L., Lamborn, S. D., Darling, N., Mounts, N. S., \& Dornbusch, S. M. (1994). Over-time changes in adjustment and competence among adolescents from authoritative, authoritarian, indulgent, and neglectful families. Child Development, 65(3), 754-770. 
Stipek, D., Milburn, S., Clements, D., \& Daniels, D. H. (1992). Parents' beliefs about appropriate education for young children.Journal of Applied Developmental Psychology, 13(3), 293-310.

Strage, A., \& Brandt, T. S. (1999). Authoritative parenting and college students' academic adjustment and success. Journal of Educational Psychology, 91(1), 146.

Street, C. (2004). examining learning to teach through a social lens: how mentors guide newcomers into a professional community of learners. Teacher Education Quarterly, 31(2), 7-24.

Stright, A. D., Gallagher, K. C., \& Kelley, K. (2008). Infant temperament moderates relations between maternal parenting in early childhood and children's adjustment in first grade. Child Development, 79(1), 186-200.

Stright, A. D., Herr, M. Y., \& Neitzel, C. (2009). Maternal scaffolding of children's problem solving and children's adjustment in kindergarten: Hmong families in the United States. Journal of Educational Psychology, 101(1), 207.

Stright, A. D., Neitzel, C., Sears, K. G., \& Hoke-Sinex, L. (2001). Instruction begins in the home: Relations between parental instruction and children's self-regulation in the classroom. Journal of Educational Psychology, 93(3), 456.

Sullivan, M. C., McGrath, M. M., \& Harrison, M. J. (1999). Proximal and distal correlates of maternal control style. Western Journal of Nursing Research, 21(3), 313-334.

Tamis-LeMonda, C. S., Kuchirko, Y., \& Song, L. (2014). Why is infant language learning facilitated by parental responsiveness? Current Directions in Psychological Science, 23(2), 121-126.

Taylor, H. B., Anthony, J. L., Aghara, R., Smith, K. E., \& Landry, S. H. (2008). The interaction of early maternal responsiveness and children's cognitive abilities on later decoding and reading comprehension skills. Early Education and Development, 19(1), 188-207.

Tenenbaum, H. R., \& Leaper, C. (2003). Parent-child conversations about science: The socialization of gender inequities? Developmental Psychology, 39(1), 34

Thompson, R. B., \& Foster, B. J. (2014). Socioeconomic status and parent-child relationships predict metacognitive questions to preschoolers. Journal of Psycholinguistic Research, 43(4), 315-333.

Trainin, G., \& Swanson, H. L. (2005). Cognition, metacognition, and achievement of college students with learning disabilities. Learning Disability Quarterly, 28(4), 261-272. 
Usai, M. C., Garello, V., \& Viterbori, P. (2009). Temperamental profiles and linguistic development: Differences in the quality of linguistic production in relation to temperament in children of 28 months. Infant Behavior and Development, 32(3), 322-330.

Valiente, C., Eisenberg, N., Haugen, R. G., Spinrad, T. L., Hofer, C., Liew, J., \& Kupfer, A. (2011). Children's effortful control and academic achievement: Mediation through social functioning. Early Education \& Development, 22(3), 411-433.

Valiente, C., Eisenberg, N., Spinrad, T. L., Reiser, M., Cumberland, A., Losoya, S. H., \& Liew, J. (2006). Relations among mothers' expressivity, children's effortful control, and their problem behaviors: A four-year longitudinal study. Emotion, 6(3), 459.

Valiente, C., Lemery-Chalfant, K., Swanson, J., \& Reiser, M. (2008). Prediction of children's academic competence from their effortful control, relationships, and classroom participation. Journal of Educational Psychology, 100(1), 67.

Vanderstoep, S. W., Pintrich, P. R., \& Fagerlin, A. (1996). Disciplinary differences in selfregulated learning in college students. Contemporary Educational Psychology, 21(4), 345362.

Vygotsky, L. (1978). Mind in society: The development of higher psychological processes. Cambridge, MA: Harvard University Press.

Weinstein, C., \& Husman, J., \& Dierking, D. R. (2000). Self-regulation interventions with a focus on learning strategies. In M. Boekaerts, P. R. Pintrich, \& M. Zeidner (Eds.), Handbook of self-regulation (pp. 727-747). San Diego, CA: Academic Press.

Welsh, J. A., Nix, R. L., Blair, C., Bierman, K. L., \& Nelson, K. E. (2010). The development of cognitive skills and gains in academic school readiness for children from low-income families. Journal of Educational Psychology, 102(1), 43.

Wertsch, J. V., McNamee, G. D., McLane, J. B., \& Budwig, N. A. (1980). The adult-child dyad as a problem-solving system. Child Development, 51, 1215-1221.

Whitebread, D., Anderson, H., Coltman, P., Page, C., Pasternak, D. P., \& Mehta, S. (2005). Developing independent learning in the early years. Education 3-13, 33(1), 40-50.

Whitebread, D., \& Basilio, M. (2012). The emergence and early development of selfregulation in young children. Profesorado, Revista de Currículum y Formación del Profesorado, 16(1), 15-34.

Whitebread, D., Bingham, S., Grau, V., Pino-Pasternak, D., \& Sangster, C. (2007). Development of metacognition and self-regulated learning in young children: Role of collaborative and peer-assisted learning. Journal of Cognitive Education and Psychology, $6(3), 433-455$. 
Wiebe, S. A., Sheffield, T., Nelson, J. M., Clark, C. A., Chevalier, N., \& Espy, K. A. (2011). The structure of executive function in 3-year-olds. Journal of Experimental Child Psychology, 108(3), 436-452.

Wigfield, A., Klauda, S. L., \& Cambria, J. (2011). Influences on the development of academic self-regulatory processes. In B.J. Zimmerman, D.H. Schunk (Eds.), Handbook of Self-Regulation of Learning and Performance (pp. 33-48). New York: Routledge.

Winne, P. H. (1996). A metacognitive view of individual differences in self-regulated learning. Learning and Individual Differences, 8(4), 327-353.

Winne, P. H. (2004). Students' calibration of knowledge and learning processes: Implications for designing powerful software learning environments. International Journal of Educational Research, 41(6), 466-488.

Winne, P. H., \& Hadwin, A. F. (1998). Studying as self-regulated learning. In D. J. Hacker, J. Dunlosky, \& A. C. Graesser (Eds.), Metacognition in Educational Theory and Practice, (pp. 277-304). Mahwah, NJ: Lawrence Erlbaum Associates.

Wolters, C. A. (1999). The relation between high school students' motivational regulation and their use of learning strategies, effort, and classroom performance. Learning and Individual Differences, 11(3), 281-299.

Wolters, C. A. (2003). Regulation of motivation: Evaluating an underemphasized aspect of self-regulated learning. Educational Psychologist, 38(4), 189-205.

Wolters, C. A., \& Pintrich, P. R. (1998). Contextual differences in student motivation and self-regulated learning in mathematics, English, and social studies classrooms. Instructional Science, 26(1-2), 27-47.

Wood, D., Bruner, J. S., \& Ross, G. (1976). The role of tutoring in problem solving. Journal of Child Psychology and Psychiatry, 17(2), 89-100.

Wood, D., \& Middleton, D. (1975). A study of assisted problem-solving. British Journal of Psychology, 66(2), 181-191.

Xu, M., Benson, S. N. K., Mudrey-Camino, R., \& Steiner, R. P. (2010). The relationship between parental involvement, self-regulated learning, and reading achievement of fifth graders: A path analysis using the ECLS-K database. Social Psychology of Education, 13(2), 237-269.

Yeung, W. J., Linver, M. R., \& Brooks-Gunn, J. (2002). How money matters for young children's development: Parental investment and family processes. Child Development, 73(6), 1861-1879. 
Zeytinoglu, S., Calkins, S. D., \& Leerkes, E. M. (2018). Maternal emotional support but not cognitive support during problem-solving predicts increases in cognitive flexibility in early childhood. International Journal of Behavioral Development, 1-12.

Zhang, D. (2005). Parent practices in facilitating self-determination skills: The influences of culture, socioeconomic status, and children's special education status. Research and Practice for Persons with Severe Disabilities, 30(3), 154-162.

Zhang, H., \& Whitebread, D. (2017). Linking parental scaffolding with self-regulated learning in Chinese kindergarten children. Learning and Instruction, 49, 121-130.

Zhang, L., Karabenick, S. A., Maruno, S. I., \& Lauermann, F. (2011). Academic delay of gratification and children's study time allocation as a function of proximity to consequential academic goals. Learning and Instruction, 21(1), 77-94.

Zhou, Q., Hofer, C., Eisenberg, N., Reiser, M., Spinrad, T. L., \& Fabes, R. A. (2007). The developmental trajectories of attention focusing, attentional and behavioral persistence, and externalizing problems during school-age years. Developmental Psychology, 43(2), 369.

Zhou, Q., Main, A., \& Wang, Y. (2010). The relations of temperamental effortful control and anger/frustration to Chinese children's academic achievement and social adjustment: A longitudinal study. Journal of Educational Psychology, 102(1), 180.

Zimmerman, B. J. (1998). Developing self-fulfilling cycles of academic regulation: An analysis of exemplary instructional models. In D. H. Schunk \& B. J. Zimmerman (Eds.), Self-regulated learning: From teaching to self-reflective practice (pp. 1-19). New York, NY: Guilford.

Zimmerman, B. J. (2000). Attaining self-regulation: A social cognitive perspective. In M. Boekaerts, P. R. Pintrich, \& M. Zeidner (Eds.), Handbook of self-regulation (pp. 13-39). San Diego, CA: Academic Press.

Zimmerman, B. J. (2002). Becoming a self-regulated learner: An overview. Theory into Practice, 41(2), 64-70.

Zimmerman, B. J. (2008). Investigating self-regulation and motivation: Historical background, methodological developments, and future prospects. American Educational Research Journal, 45(1), 166-183.

Zimmerman, B. J., \& Martinez-Pons, M. (1988). Construct validation of a strategy model of student self-regulated learning. Journal of Educational Psychology, 80(3), 284.

Zimmerman, B. J., \& Schunk, D. H. (2008). Motivation An essential dimension of selfregulated learning. In D. H. Schunk \& B. J. Zimmerman (Eds.). Motivation and self- 
regulated learning: Theory, research, and applications (pp. 1-31). New York: Lawrence Erlbaum Associates.

Zimmerman, B. J., \& Schunk, D. H. (2011). Self-regulated learning and performance. An introduction and an overview. In B. J. Zimmerman, D. H. Schunk (Eds.), Handbook of Self-Regulation of Learning and Performance (pp. 1-12). New York: Routledge. 